\title{
Actin Filaments and Bundles in Flow
}

\author{
Dissertation \\ zur Erlangung des Doktorgrades \\ der Mathematisch-Naturwissenschaftlichen Fakultäten \\ der Georg-August-Universität zu Göttingen
}

vorgelegt von

Dagmar Regine Steinhauser

aus Schwäbisch Hall

Göttingen 2008 
D 7

Referent: Prof. Dr. Tim Salditt

Koreferent: Prof. Dr. Stephan Herminghaus

Tag der mündlichen Prüfung: 29. Mai 2008 


\section{Zusammenfassung}

Das Protein Aktin ist eine wichtige Komponente des Zytoskeletts und wird in vivo in Filamente, Bündel und Netzwerke organisiert. Aktinfilamente gelten als Modellsysteme für semiflexible Polymere um grundlegende Probleme und Fragestellungen in der Polymerphysik zu beantworten. In der hier vorliegenden Arbeit werden Experimente zum Verhalten einzelner semiflexibler Filamente in mikrofluidischer Umgebung vorgestellt. Des Weiteren werden Methoden der Mikrofluidik zur Analyse der zeitlichen Entwicklung des Entstehens von Aktinbündeln verwendet.

Der erste Teil dieser Arbeit beschäftigt sich mit den Eigenschaften der Aktinfilamente in Mikrokanälen im Fluss. An verschiedenen Stellen entlang eines Querschnitts des Kanals werden Filamente bei unterschiedlichen Geschwindigkeiten analysiert. Die fluoreszentmarkierten Filamente werden mikroskopisch abgebildet. Dabei ermöglicht eine Laseranregung kurze Belichtungsdauern. Die semiflexible Eigenschaft der Filamente ist in den durchgeführten Experimenten von besonderer Bedeutung aufgrund der Tatsache, dass die Kanaldimensionen (Kanalbreite und Kanaltiefe) in etwa der charakteristischen Längen der Aktinfilamente (Konturlänge und Persistenzlänge) entsprechen. Durch den Fluss im Mikrokanal werden die Aktinfilamente gestreckt oder gebogen, wobei gestreckte Filamente häufiger zu beobachten sind. Diese sind außerdem nahezu in Flussrichtung ausgerichtet. Die Wahrscheinlichkeitsverteilungen des End-zuEnd-Abstandes und des Winkels des End-zu-End-Vektors relativ zur Flussrichtung bei unterschiedlichen Geschwindigkeiten zeigen, dass die Streckung und Ausrichtung mit zunehmenden Geschwindigkeiten ansteigt. Die charakteristischen Orientierungsparameter, der bevorzugte Winkel und die Breite der Wahrscheinlichkeitsverteilung des Winkels, gehorchen den Skalengesetzen für steife oder stark gestreckte Polymere im einfachen Scherfluss. Zusätzlich zu den gestreckten Filamenten kann teilweise auch eine Taumelbewegung beobachtet werden, bei der ein Ende eines Filaments das andere überholt. Die Krümmungsradien der gebogenen Filamente während des Taumelns nehmen mit ansteigenden Geschwindigkeiten ab. Unter Verwendung des Kräftegleichgewichts von Reibungs- und Biegekräften wird ein Skalengesetz hergeleitet, mit welchem die experimentellen Werte äußerst gut beschrieben werden können. Gebogene Filamente werden zusätzlich auch in der Kanalmitte beobachtet, deren relativ stabile Formen durch Parabeln beschrieben werden können. Der Grund für die Verbiegung dieser Filamente ist das nicht-monotone, parabolische Flussprofil. Die parabelförmigen Konturen können mit einem Model eines elastischen Stabes beschrieben werden. 
Zusätzlich zu den Konformationen und Orientierungen der Filamente im Fluss wird die Häufigkeitsverteilung der Filamente an verschiedenen Kanalpositionen entlang eines Querschnittes analysiert. Wahrscheinlichkeitsverteilungen des Schwerpunktes zeigen, dass die Filamente nicht gleichmäßig über die ganze Kanalbreite verteilt sind. In Nähe der Wände existieren deutliche Verarmungszonen. Bei hohen Flussgeschwindigkeiten bildet sich zusätzlich eine starke Verarmungszone in der Kanalmitte aus. Eine Verringerung der Filamentdiffusivität hin zur Wand ist aufgrund der ansteigenden Scherrate vorhanden. Dies führt zu einer Bewegung der Filamente weg von der Kanalmitte. In Nähe der Wände lassen sich die Verarmungszonen durch sterische und hydrodynamische Wechselwirkungen mit den Wänden erklären. Die ortsabhängige Diffusivität der Filamente kann bestimmt werden indem eine Proportionalität zwischen Diffusivität und mittlerer quadratischer Abweichung der Filamentsegmente von der Stromlinie des Filamentsschwerpunktes angenommen wird. Unter Verwendung dieser Diffusivität können die Wahrscheinlichkeitsverteilungen des Schwerpunktes bei verschiedenen Geschwindigkeiten berechnet werden. Die berechneten und experimentellen Verteilungen zeigen die gleichen grundlegenden Merkmale wie Verarmungszonen an den Wänden und in der Kanalmitte. Im Grenzfall für hohe Geschwindigkeiten erlaubt das Modell sogar eine quantitative Beschreibung.

Im zweiten Teil der Arbeit wird die Bildung von Bündeln bestehend aus Aktinfilamenten unter Zugabe bündelbildender Proteine oder mehrwertiger Ionen diskutiert. Mit Methoden der Mikrofluidik basierend auf hydrodynamische Fokussierung wird die Bündelung zeitaufgelöst auf molekularer Skala fluoreszensmikroskopisch abgebildet. Ausgehend von einzelnen Filamenten wird die Bildung der Bündel und deren Anwachsen analysiert. Aus der Intensität des Fluoreszenssignals kann dabei die Anzahl der Filamente innerhalb eines Bündels bestimmt werden. Die Auswertung zeigt, dass die Bündlung von Aktinfilamenten ein diffusionslimitierter Prozess ist. Durch Analyse der thermischen Fluktuationen der Bündel lassen sich auch ihre mechanischen Eigenschaften bestimmen. Die Abhängigkeit der gemessenen Persistenzlänge von der Anzahl der Filamente innerhalb eines Bündels weißt auf eine schwache Kopplung der Filamente untereinander hin. Diese Kopplung resultiert vermutlich aus einer Optimierung der Ladungsverteilung innerhalb der Bündel.

Die Ergebnisse der vorliegenden Arbeit zeigen, dass die Kombination von Mikrofluidik und Fluoreszensmikroskopie eine leistungsfähige Methode zur Untersuchung von Bündel- oder Netzwerkbildungen ist. Besonders beachtenswert ist die Möglichkeit einer zeitaufgelösten Beobachtung und Analyse, die ein sehr großes Potential der Erforschung anderer zeitabhängiger Prozesse, wie beispielweise von enzymatischen Reaktionen und Polymerisationen hat. 


\section{Abstract}

Actin, a protein and major component of the cytoskeleton, is organized in vivo into filaments, bundles, and networks which play an important role in mechanical stability and cellular motility. Aside from their biological relevance, actin filaments can be used as model systems for semiflexible polymers to answer fundamental physical questions in polymer science. In the presented thesis, results are discussed which consider the behavior of single semiflexible polymers in the field of microfluidics. Moreover, microfluidic tools are also used to study the bundling of actin filaments in vitro.

In the first part of this thesis, the behavior of semiflexible actin filaments in flow inside microchannels is investigated. The filaments are analyzed at different channel positions along a cross-section for different flow velocities. To this end, fluorescently labeled actin filaments are observed by using fluorescence microscopy for which a laser illumination enables short exposure times. In order to gain results for which the semiflexible nature of actin filaments is significant, the channel dimensions (width and depth) are adapted to have approximately the same size as the characteristic lengths of the filaments (persistence length and contour length). The results indicate that the microflow causes either elongation or bending of filaments. Predominately, the filaments are elongated. The elongated filaments are nearly aligned in the flow direction. The elongation and alignment increase with larger flow velocities as it is seen in the end-to-end distance probability distributions and the angle probability distributions. The characteristic parameters of the filament orientation, the preferred angle and the width of the angle probability distribution, obey scaling laws which are known for strongly elongated or stiff polymers in simple shear flow. In addition to elongated filaments, tumbling of filaments is observed. The radii of curvature of bent filaments during tumbling decrease with increasing velocities. By balancing drag forces and bending forces, a scaling is derived with which the experimental values can be described. Additionally, bent filaments are also found at the channel center. In this region, the bent shapes are stable and can be quantified by parabolas. These bent conformations can be related to the non-monotonic parabolic velocity field, and the parabolic profiles can be reconstructed by modeling filament as elastic rods.

Additionally to conformational and orientational studies of actin filaments in flow, an important point of interest is the channel positions along the cross-section at which filaments are most frequently found. The center-of-mass probability distributions show that the filaments are not equally distributed over the channel width. For large velocities, filaments are less frequently found near the channel center. Furthermore, depletion 
layers near walls are observed. Consequently, filaments migrate away from the channel center as well as away from the channel walls. The cross-streamline migration away from the channel center can be explained by a decrease of the filament diffusivity toward the walls due to an increase of the shear rate. Near walls, steric and hydrodynamic interactions with the walls lead to depletion layers. To quantify the spatially-varying diffusivity, the segment distributions of filaments at different channel positions as well as for different velocities are analyzed. Assuming proportionality between the diffusivity and the mean square deviation of segments from the center-ofmass streamline of filaments, the diffusivity at each channel position for a certain flow velocity can be determined. Using this diffusivity, a governing equation for the centerof-mass probability distribution is set up in which the spatially-varying diffusivity and hydrodynamic interactions with the walls can generate drift on the filaments. The calculated and measured distributions show the same essential characteristics like depletion layers at walls and the channel center. For large velocities, a nearly quantitative agreement is obtained.

The second part of this thesis considers the actin bundling in the presence of linker molecules. Using microfluidic tools, a method is developed in order to observe the bundling of actin filaments in situ at a molecular level. The bundles are imaged by fluorescence microscopy and the intensity of the emitted light from a bundle is a measure for the number of filaments inside the bundle. Usage of a hydrodynamic focusing device enables a time-resolved visualization of the bundling from single filaments to thick bundles. As a result, it is shown that bundling is a diffusion-limited process. Furthermore, the analysis of thermal fluctuations of bundles characterizes their mechanical properties and a relation between the persistence length and the number of filaments is obtained. This relation suggests a weak coupling between filaments inside bundles, probably induced by the electrostatic nature of actin.

The results presented in this thesis show that the combination of microfluidics and fluorescence microscopy is a powerful tool to investigate the kinetics of the actin bundling at a molecular scale. More generally, the time-resolved visualization of the step-by-step process has a large potential for studies of any bundling or network formation, and also for other time-dependent processes such as enzymatic reactions or polymerizations. 


\section{Contents}

Chapter 1 Introduction 1

1.1 Microfluidics of Dilute Polymer Solutions 2

1.1.1 Cross-Streamline Migration 3

1.1.2 Actin Filaments: a Model System for Semiflexible Polymers 4

1.2 Actin Networks and Bundles 5

Chapter 2 Materials and Methods 9

2.1 Soft Lithography 9

$\begin{array}{lll}2.2 & \text { Sample Preparation } & 11\end{array}$

$\begin{array}{lll}2.3 & \text { Liquid Pumping System }\end{array}$

$\begin{array}{lll}2.4 & \text { Fluorescence Microscopy } & 14\end{array}$

$\begin{array}{lll}2.5 & \text { Actin Polymerization } & 17\end{array}$

$\begin{array}{lll}2.6 & \alpha \text {-Actinin } & 18\end{array}$

Chapter 3 Worm-Like Chain Model 19

$\begin{array}{lll}3.1 & \text { Persistence Length } & 19\end{array}$

$\begin{array}{ll}3.2 \text { Worm-Like Chain } & 20\end{array}$

$\begin{array}{ll}\text { Chapter } 4 \text { Polymers in Microflow } & 23\end{array}$

4.1 Reynolds Number 23

4.2 Motion of Brownian Particles 24

$\begin{array}{lll}\text { 4.2.1 Drag Force } & 24\end{array}$

4.2.2 Diffusion 25

$\begin{array}{lll}\text { 4.2.3 Hydrodynamic Interactions } & 26\end{array}$

$\begin{array}{lll}4.3 & \text { Polymers in Shear Flow } & 27\end{array}$

4.3.1 Weissenberg Number 27

4.3.2 Dumbbell Model 28

4.3.3 Dumbbell in Simple Shear Flow 31

4.4 Cross-Streamline Migration 33

$\begin{array}{lll}\text { 4.4.1 Inertial Migration } & 34\end{array}$

4.4.2 Migration of Polymers 39 
Chapter 5 Semiflexible Filaments in Pressure-Driven Flow

5.1 Experiment 45

5.2 Data Analysis 48

5.3 Relaxation of Actin Filaments 50

5.4 Conformations of Actin Filaments 52

5.4.1 Orientation 52

5.4.2 Elongation and Tumbling 56

5.4.3 Conformations at the Centerline 61

5.5 Center-of-Mass Probability Distribution 65

5.6 Curved Channels 79

$\begin{array}{lr}\text { Chapter } 6 \text { Actin Bundles } & 87\end{array}$

6.1 Mechanisms of Actin Bundle Formation 87

6.2 Bending of Actin Bundles 88

6.3 Fluctuating Actin Bundles 90

6.3.1 Experiment and Analysis 90

6.3.2 Persistence of Actin Bundles 93

$\begin{array}{lll}6.4 & \text { Bundling Kinetics } & 97\end{array}$

6.4.1 Hydrodynamic Focusing 97

6.4.2 Evolution of Actin Bundling 98

6.4.3 Outlook 105

Chapter 7 Conclusions $\quad 108$

Chapter 8 Appendix 113

8.1 Calculation of the Center-of-Mass Probability Distribution 113

$\begin{array}{lll}8.2 & \text { Bibliography } & 115\end{array}$ 


\section{Chapter 1}

\section{Introduction}

The field of microfluidics aims to develop techniques to manipulate, control, and analyze chemical reactions and physical as well as biological processes on the microand nanoscale. To this end, the behavior of small fluid volumes is studied. The popular idea of a "lab-on-a-chip" is to integrate several laboratory operations, for example sample preparation and detection, into a single microfluidic device. Due to the miniaturization of the system, samples that are expensive or difficult to obtain can be handled. Additionally, integration of several steps into one microfluidic device enables faster analysis. The possibility of single particle detection increases the measurement sensitivity. Furthermore, important conditions of processes, such as concentrations or temperature, can be controlled precisely. It is conceivable that in the future integrated, portable clinical diagnostic devices for home use will replace time-consuming laboratory analysis. Nowadays, many microfluidic applications are already available including techniques for DNA sequencing, polymerase chain reaction, cell sorting, and cell culture $[1,2]$.

Typical micro- and nanofluidic devices have a length scale comparable to the dimension of many components involved in fundamental biological processes such as several biopolymers (e.g. DNA or actin). Microfluidics are therefore a powerful tool for manipulating and studying individual biopolymers, and experiments and techniques can be performed which are not possible at macroscopic scales [3]. Furthermore, microfluidic devices can mimic conditions that exist in biological systems. For example, the flow and geometry of blood vessels can be modeled similar to physiological conditions [4].

Microchannels can also be used to study effects due to confinement, which is often found in biological systems such as for individual actin filaments confined by the dense cytoskeleton network inside cells $[5,6]$. The cytoskeleton of cells is a complex polymer network which plays an important role in cellular motility and mechanical stability. By 
decreasing the complexity of the system by performing in vitro experiments with only a few components, the mechanical properties of the different components and their interactions can be analyzed. This helps to identify their biological relevance and to obtain a more precise picture of composition, properties, behavior, and functionalities of the cytoskeleton.

\subsection{Microfluidics of Dilute Polymer Solutions}

The physical behavior of a fluid with only a nano- or picoliter in volume can differ dramatically from that known at the macroscopic scale. One important property at the microscale is the Reynolds number, which is the ratio of inertial forces to viscous forces. In microfluidic channels, the Reynolds number is typically very small and the flow is laminar. Thus, flow fields for Newtonian fluids are deterministic and in principle predictable, which simplifies the handling of fluids in several aspects. On the other hand, the laminar flow can also be a disadvantage because mixing only occurs by diffusion. For efficient mixing, techniques are required which increase the interfaces between the fluids [7]. The behavior of fluids in microfluidic devices are also dominated by fluidic resistance, surface tension, and an increased surface area to volume ratio [8]. In addition, so-called complex fluids, to which polymer solutions belong, can behave quite differently compared to simple Newtonian fluids. For example, the flow can become chaotic, even at small Reynolds numbers, for dilute polymer solutions by a phenomenon called elastic turbulence $[9,10]$. Since polymers are involved in many microfluidic applications, for example in DNA or protein analysis [3], knowledge about the behavior of polymers in microfluidic devices is important. Material transport properties, orientation, and configuration are useful for designing new devices and developing new microfluidic techniques. In order to increase the understanding, many experiments have been performed by observing the behavior of polymers in different flow fields [11-16]. In particular, the conformation and orientation of DNA molecules (e.g. $\lambda$-DNA with persistence length about $50 \mathrm{~nm}$ and contour length about $16.5 \mu \mathrm{m}$ [17]) have been investigated extensively in many flows, including elongational [14, 18], shear [11-13], a combination of both elongational and shear [16], oscillatory [15], and Poiseuille [19]. As a result it has been shown that the DNA molecules in shear flow stretch and align in the flow direction [12] and additionally tumble due to thermal noise or velocity fluctuations [11, 20]. However, little is known about the behavior of other biopolymers in microflow, which cannot necessarily fit into the DNA descriptions. There are interesting questions regarding the semiflexible actin, which are discussed in this thesis. 


\subsubsection{Cross-Streamline Migration}

Besides the orientational and configurational behavior of polymers in different flow fields, the positions of polymers inside microchannels are an important point of interest for microfluidic applications. Due to cross-streamline migration of polymers under flow, the concentration of polymers along a cross-section of a channel is not constant. There is experimental evidence of depletion layers near confining walls in curvilinear [21-23] and rectilinear geometry [23-25]. However, predictions from different theoretical and computational studies for polymers in microchannels are controversial, even in answering fundamental questions such as the direction of migration toward or away from walls [23, 26, 27]. 50 years ago, a decrease of the depletion layers with larger shear rate was assumed because the alignment and elongation of the polymers in flow reduce the steric hindrance to approach a wall [23]. However, it was experimentally found that for flexible polymers depletion layers near a single wall increase with higher flow strength [25].

Thermodynamic arguments predict migration toward the channel center for Poiseuille flow and therefore describe correctly the increase of depletion layers near the walls [23, 28]. For this special flow field, the local shear rate has a minimum at the channel center and decreases toward the walls. Thus, polymers near walls are more stretched than polymers at the channel center. Since the configurational entropy is decreased for a stretched polymer, the entropy is largest at the channel center. This leads to the above mentioned assumption of migration toward the channel center, and the probability to find a polymer at the channel center should be largest. However, some computer simulations disagree with this prediction and they suggest, additionally to depletion layers at the walls, a local minimum in the probability distribution of the center-of-mass of the polymers at the channel center [29, 30]. Furthermore, migration is always caused by position-dependent configurations of polymers across the channel in thermodynamic theories, and therefore no migration is predicted in uniform flow. However, experiments [23] and computer simulations [30] demonstrate that depletion layers near walls exist even in the case of uniform shear flow. Consequently, thermodynamic theories cannot describe migration of polymers completely. In a flowing polymer solution in microchannels, thermodynamic arguments should also be treated carefully [27, 31]. These systems are not at equilibrium and an external force is needed to describe the flow scenario.

Recently, it has been shown in computer simulations [29, 30] that hydrodynamic interactions of polymers with walls are needed in order to understand cross-streamline migration in microfluidic devices. Generally, in kinetic theories two different sources 
for migration in straight channels have been predicted [26, 29]. Near a wall, a deterministic migration is generated by broken symmetry of hydrodynamic interactions, which leads to migration of polymers away from walls [26, 29]. This is consistent with the experimental results of increased depletion layers near a single wall found for DNA molecules [25]. Additionally, Brownian migration [26, 29, 32, 33] is expected in inhomogeneous flow due to a coupling of a spatial-varying diffusion of polymers with hydrodynamics which leads, for Poiseuille flow, to a migration away from the channel center [29]. So far, no measurements show an experimental evidence for Brownian cross-streamline migration in straight channels.

\subsubsection{Actin Filaments: a Model System for Semiflexible Polymers}

The behavior of flowing polymers in microchannels is not only determined by the flow field and channel geometry, but certainly also by the properties of the polymers. An important property to quantify the polymer stiffness is the persistence length $L_{p}$, which is the distance over which the segment orientations are correlated. For flexible polymers the persistence length is small in comparison to the contour length $L\left(L>>L_{p}\right)$, and for stiff polymers it is large $\left(L<<L_{p}\right)$. In an intermediate regime for which the persistence length is approximately equal to the contour length $\left(L \approx L_{p}\right)$, the polymer is described as semiflexible.

Our experiments are performed with actin filaments, a component of the cytoskeleton of cells, which is often used as a model system for semiflexible polymers [5, 34]. Actin filaments have a persistence length of about $13 \mu \mathrm{m}[5,6]$. In vitro, the average length of actin filaments is typically $5-20 \mu \mathrm{m}$ [35], depending strongly on conditions during the polymerization process. Thus, actin filaments belong indeed to the class of semiflexible polymers. However, a polymerized actin solution in vitro is always polydisperse and the lengths of the filaments vary. They can be up to $100 \mu \mathrm{m}$ long [36]. Consequently, looking only at very long $\left(L>>L_{p}\right)$ or very short $\left(L<<L_{p}\right)$ filaments, actin can also be used as a model system for flexible or stiff polymers. Because the persistence length of actin is several micrometers long, the contour can be observed with microscopy techniques. Thus, conformations and dynamics of actin can be studied directly. The possibility to visualize the contour is a key advantage of actin over synthetic polymers, which have typically persistence lengths of only a few nanometers [37].

Most experimental studies in microfluidics have been performed with flexible polymers (often DNA [9-16, 19-22, 25, 38] ) or stiff polymers (e.g. microtubules [39, 40]). In this thesis, the behavior of semiflexible polymers is investigated in pressure-driven flow. To obtain specific features in the behavior of the polymers resulting from their semiflexible 
nature, the relevant length scales are chosen to have almost the same size. More specifically, the characteristic lengths of the polymers (contour length and persistence length) are commensurate with the channel dimensions (width and depth). The orientation and conformation of the polymers are analyzed at different channel positions. Furthermore, the center-of-mass probability distribution is measured along a crosssection of the channel in order to study cross-streamline migration of polymers over the entire channel. In addition to the investigations of single actin filaments in microflow, microfluidic tools are also used in this thesis to bundle actin in situ.

\subsection{Actin Networks and Bundles}

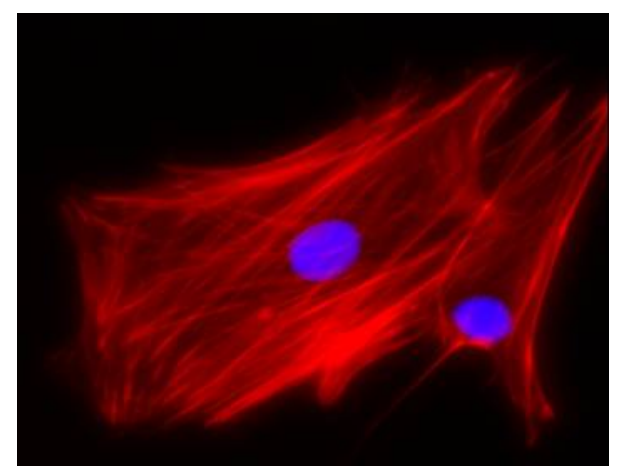

Figure 1-1: Actin networks in muscle cells (red color: actin, blue color: cell nucleus) [5].

Actin is one of the three major components of the cytoskeleton of cells and plays an important role for determining the cell shape, providing mechanical support, and enabling cell motility and cell division [41]. Actin is one of the most abundant proteins in eukaryotic cells. In muscle cells, actin is, together with myosin, the major protein and makes up about $10 \%$ [41] of the weight of the total cell proteins. Even in non-muscle cells, actin comprises 1-5\% [41]. Actin is also one of the most highly conserved proteins differing by no more than $5 \%$ between species [41]. Actin monomers are globular proteins consisting of approximately 375 amino acids (43kD). The monomers can polymerize into filamentous actin, which are thin helical fibers of approximately $7 \mathrm{~nm}$ in diameter and up to several micrometers in length. Actin filaments have polarity and their ends (designated as minus and plus end) can be distinguished because the actin monomers are all orientated with their cleft end in one direction (toward the minus end). Monomers can bind to both end of filaments but filaments grow faster at the plus end. The polymerization is reversible and filaments can depolymerize by dissociation of monomers. Both actin filaments and monomers are found in vivo. Polymerization and 
depolymerization inside a cell, depending on many of different factors, determine the length distribution of the filaments.

Within a cell, actin filaments are organized in bundles and three-dimensional networks.

Bundles are closely packed parallel arrays of actin filaments, whereas actin networks are loosely packed three-dimensional, often orthogonal, meshworks. The actin cytoskeleton is a dynamic system, where filaments shrink or grow in length, and bundles and networks are formed or dissolved. This fast modification in their organization can lead to large changes in the cell shape.

The crosslinking into bundles and networks as well as the polymerization and depolymerizition of actin are regulated by a large number of different actin-binding proteins (ABPs). Over 150 ABPs are known, accounting for approximately $25 \%$ of cellular proteins [42]. There are ABPs that bind to monomers, but some also bind to filaments. The roles of ABPs include sequestering, capping, cutting, and stabilizing actin filaments. Furthermore, some ABPs crosslink actin filaments in higher ordered structures. In the following discussion, we focus on these actin crosslinking proteins and show some examples of actin arrangements inside cells. Typically, actin crosslinking proteins have two actin binding sites and their length and flexibility determine whether predominantly bundles or networks are formed. Short rigid proteins force actin filaments to lie close together. Parallel aligned arrays of actin filaments are formed and result in actin bundles. In contrast, long flexible proteins can arrange actin filaments in a more complex manner with more distance between them, resulting in networks.

a)

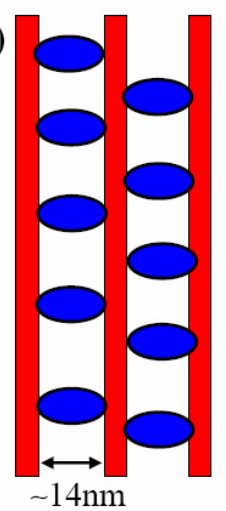

b)

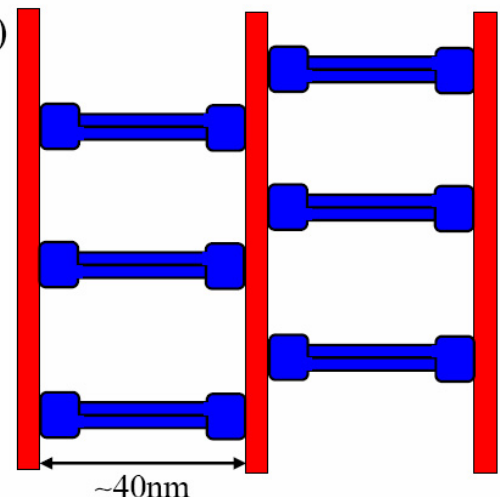

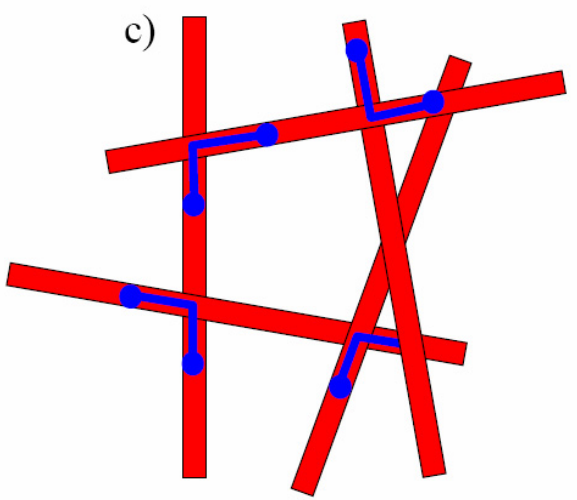

Figure 1-2: Actin bundles and networks. a) Dense actin bundle formed by fimbrin. b) Loosely spaced actin bundle formed by $\alpha$-actinin. c) Actin network formed by filamin.

Within a cell, the highest concentration of actin filaments is beneath the plasma membrane [41], where the filaments are organized in a network called the cell cortex. One ABP involved in this network is filamin, which binds two orthogonal actin filaments together and create a loose three-dimensional network (figure 1-2c). The 
structure of the actin network varies in different cells, but the main task of the cell cortex is the same for all cells: to determine and to stabilize the cell shape. Furthermore, the cell cortex is involved in a variety of surface activities such as cell movement.

Another example of actin organization is the formation of dense actin bundles, which exist in fingerlike membrane projections like microvilli [41]. In microvilli, the actin filaments are closely packed due to the ABPs fimbrin and villin (figure 1-2a). The stiff actin bundles support the fragile projecting membrane. The major effect is the increase of the surface of cells, which facilitates absorption and secretion.

More loosely spaced actin bundles are formed by the ABP $\alpha$-actinin (figure 1-2b). These actin bundles build, for example, the contractile ring during cell division. The increase of bundle spacing allows interactions with the motor protein myosin. The motor protein myosin can "walk" along actin filaments, driven by hydrolysis of ATP, and contraction and deformation can occur. Besides cell division, interactions of actin and myosin are responsible for a variety of cellular movements, in particular for muscle contraction.

To better understand the behavior and role of actin bundles and networks, in vitro experiments have been performed [42-53]. In vitro, actin filaments can be crosslinked by ABPs [42, 43], by counterion condensation in the presence of multivalent ions [44, $45]$, and by depletion forces in the presence of coiled polymers [54, 55]. Using bulk rheology measurements, visoelastic properties of actin networks have been investigated for semi-dilute entangled actin networks [47, 48], concentrated gel-like actin networks $[46,49]$ and crosslinked actin networks [50-53]. Microrheology, where the thermal motion of small particles inside a network is analyzed, has been used to increase the microscopic understanding of actin networks [56, 57]. To obtain information about the structure of actin networks, solutions have been studied mainly using x-ray scattering [58-62], (confocal) fluorescence microscopy [57, 63], electron microscopy [62, 64], and optical birefringence $[65,66]$.

The crosslinking and bundling of actin filaments have been usually observed in bulk solutions, having a large number of filaments and crosslinker molecules inside the solution. Mainly, the macroscopic behavior has been analyzed. Only a few experiments have considered the behavior of individual actin bundles formed by ABPs [67] and the behavior of filaments in a network at a mesoscopic length scale [68]. In this thesis, dynamics of individual bundles, consisting of only a few actin filaments, are investigated. Furthermore, experiments are performed to study the kinetics of actin bundling. Using microfluidic tools, we are able to observe the time-resolved evolution of actin bundles starting with a large number of individual actin filaments and ending with only a few thick bundles. 


\section{Chapter 2}

\section{Materials and Methods}

\subsection{Soft Lithography}

We fabricate the microfluidic devices using soft lithography [69, 70]. This technique consists of two basic steps. Firstly, a master is produced by photolithography (see figure 2-1). In a second step, a negative replica of the master is made using an elastomeric polymer (see figure 2-2).

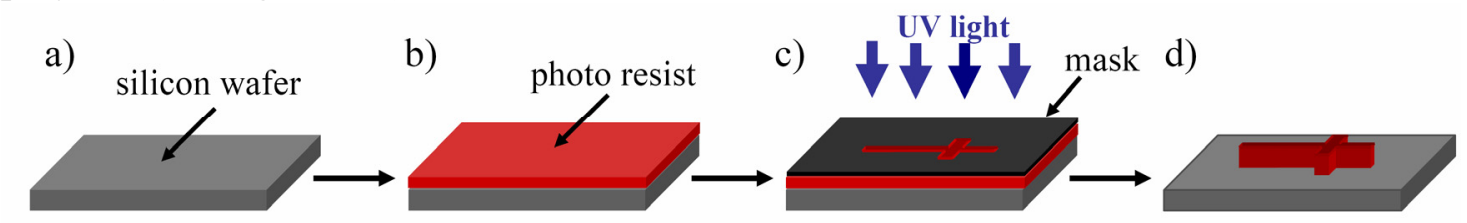

Figure 2-1: Photolithography. a) Clean silicon wafer. b) Spincoated photo resist. c) Illumination with UV light through a mask. d) Master.

a)

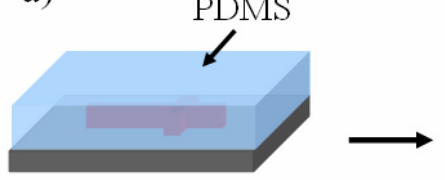

b)

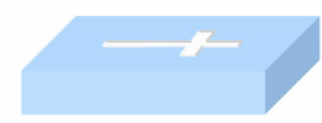

c)

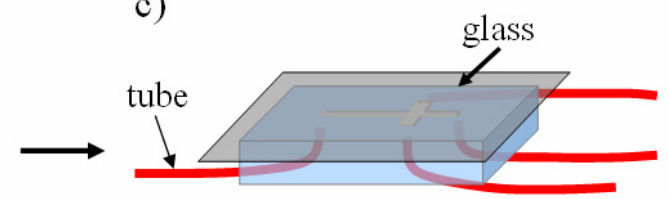

Figure 2-2: Fabrication of a PDMS replica. a) PDMS poured on the master. b) Open microstructure in PDMS. c) Microfluidic device.

For fabrication of the master, a photo resist is spincoated onto a silicon wafer (figure 2-1b). After connecting a mask with the photo resist, the resist is illuminated by UV light and the exposed areas are crosslinked (figure 2-1c). Removing all not-illuminated photo resist with a solvent, a master is obtained consisting of a patterned resist on a silicon wafer (figure 2-1d). To produce a replica of the master in PDMS (poly(dimethlysiloxane)), the PDMS-precursor is mixed with a crosslinker and poured onto the master (figure 2-2a). After curing, the PDMS can be peeled off (figure 2-2b). 
The resulting open microstructure can be covered with a cover slip, tubes can be added, and the microfluidic can be used for experiments (figure 2-2c).

The major advantage using soft lithography is the fast prototyping of microfluidic devices, that allows in principle the realization of new designs in $24 \mathrm{~h}[70,71]$. Once the more time-consuming step of producing a master has been made, the master can be used many times for making microfluidic devices in PDMS. Furthermore, PDMS is inexpensive, chemical inert, flexible, and transparent [69]. The surface can be functionalized and bond to other materials such as glass [72]. For example, covering the channels with a cover slip, which is useful for many microscopy experiments, is easily achieved by oxidation.

In the experiments, the microchannels have a depth of about $10 \mu \mathrm{m}$ (experiments in chapter 5) or about $20 \mu \mathrm{m}$ (experiments in chapter 6). The photo resist SU-8 2005 or the more viscous resist SU-8 50 (Micro Resist Technology GmbH, Berlin) is used for fabrication of the masters to obtain the wanted height of the microstructures. The lithography parameters for fabrication of the masters are specified in table 1.

\begin{tabular}{|c|c|c|}
\hline & SU-8 2005/ $10 \mu \mathrm{m}$ & SU-8 50/ $20 \mu \mathrm{m}$ \\
\hline spin coating: Omnicoat & $\begin{array}{l}\text { in } 5 \mathrm{~s} \text { to } 500 \mathrm{rpm} \\
\text { in } 7 \mathrm{~s} \text { to } 2000 \mathrm{rpm} \\
30 \mathrm{~s} @ 2000 \mathrm{rpm}\end{array}$ & - \\
\hline baking & $1 \mathrm{~min} @ 200^{\circ} \mathrm{C}$ & - \\
\hline spin coating: photo resist & $\begin{array}{l}\text { in } 5 \mathrm{~s} \text { to } 500 \mathrm{rpm} \\
\text { in } 1 \mathrm{~s} \text { to } 800 \mathrm{rpm} \\
60 \mathrm{~s} @ 800 \mathrm{rpm}\end{array}$ & $\begin{array}{l}\text { in } 10 \mathrm{~s} \text { to } 700 \mathrm{rpm} \\
\text { in } 30 \mathrm{~s} \text { to } 4500 \mathrm{rpm} \\
30 \mathrm{~s} @ 4500 \mathrm{rpm}\end{array}$ \\
\hline soft baking & $\begin{array}{l}1 \min @ 65^{\circ} \mathrm{C} \\
4 \min @ 95^{\circ} \mathrm{C}\end{array}$ & $\begin{array}{l}10 \mathrm{~min} @ 65^{\circ} \mathrm{C} \\
45 \mathrm{~min} @ 95^{\circ} \mathrm{C}\end{array}$ \\
\hline $\begin{array}{l}\text { exposure } \\
\left(\lambda=365 \mathrm{~nm}, 15 \mathrm{~mW} / \mathrm{cm}^{2}\right)\end{array}$ & $28 \mathrm{~s}$ & $30 \mathrm{~s}$ \\
\hline post baking & $6 \min @ 95^{\circ} \mathrm{C}$ & $10 \min @ 95^{\circ} \mathrm{C}$ \\
\hline
\end{tabular}

Table 1: Parameters used for photolithography.

The silicon wafer is cleaned with isopropanol and dried with nitrogen. In the case of using SU-8 2005, a thin layer of Omnicoat (Micro Resist Technology GmbH) is spincoated onto the silicon wafer and baked. The thin layer of Omnicoat improves the adhesion of the resist to the silicon wafer. For the more viscous resist SU-8 50, this intermediate step is not necessary. After this preparation, a thin layer of photo resist is spincoated onto the wafer and baked again. Using a mask aligner (MJB3; Süss Microtech AG, Garching), a lithography mask is brought in contact with the resist and 
illuminated with UV light. Depending on the size of the structures, a chrome mask (ML\&C, Jena-Maue) or a lithography transparency (JD-Photo-Tools Ltd., Oldham, UK) is used. The chrome mask has a higher resolution and is taken for structures $\leq 10 \mu \mathrm{m}$. For larger structures, the resolution of a lithography transparency is sufficient. For completely crosslinking the exposed areas, the photo resist is baked again. After developing using SU-8 developer (Micro Resist Technology GmbH), the master consisting of micostructures of photo resist on a silicon wafer is obtained.

The master is treated with heptafluoropropyl-trimethylsilane (97\%, Sigma Aldrich, München) to ensure a hydrophobic surface from which the PDMS can easily be pulled off. Therefore, the master is placed together with $10 \mu 1$ silane in a desiccator, the system is evacuated for 1 hour, and the silane absorb from the gas phase to the surface. Sylgard 184 PDMS (Dow Corning GmbH, Wiesbaden) is mixed with his crosslinker and thoroughly poured onto the master. To remove air bubbles inside, the sample is placed again in a desiccator and the system is evacuated until no bubbles are visible anymore. The sample is baked for 4 hours at $60^{\circ} \mathrm{C}$ to crosslink the PDMS. Finally, the PDMS is pealed off from the master.

The PDMS is bonded to a cover slip to obtain a closed microfluidic device. Before the bonding, holes are punched with a blunt needle into the filling areas. Then, the PDMS is put together with a cover slip into a plasma cleaner (Harrick Plasma, Ithaca, USA). Plasma is created for $10 \mathrm{~s}$ at a pressure of 2 mbar and the surfaces are oxidized. Bringing both surfaces immediately after oxidation in contact, a covalent bond between glass and PDMS is formed. Polyethylene tubes (inner diameter: $0.28 \mathrm{~mm}$, outer diameter: $0.61 \mathrm{~mm}$; Smiths Medicas International Ltd, UK) are glued into the connection holes using a special two-component glue for plastics (Loctite 406; Novodirect, Kehl/Rhein). The microfluidic device is now ready-for-use.

\subsection{Sample Preparation}

To prevent absorption of actin filaments at the walls, the channels are coated before the experiment. For experiments with single actin filaments, the channels are rinsed with a $1 \mathrm{mg} / \mathrm{ml}$ BSA (bovine serum albumin; Sigma Aldrich, München) solution. BSA saturates the surfaces and prevents thereby non-specific protein absorption. Additionally a $1 \mathrm{mg} / \mathrm{ml}$ BSA solution is added 1:10 to the actin solution of the experiment. Unfortunately, actin bundles stick to the BSA-coated surfaces, and a more complicated polyelectrolyte multilayer coating is used for actin bundling experiments (see figure 2-3). 


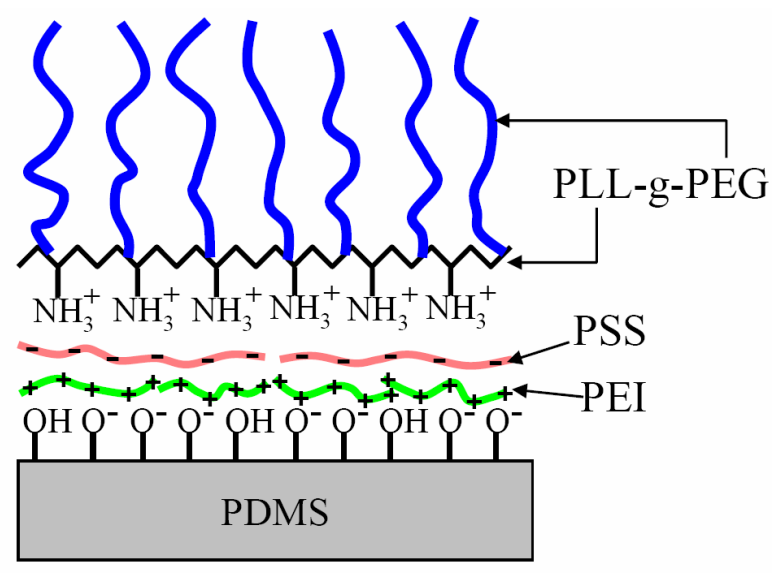

Figure 2-3: Polyelectrolyte multilayer coating.

Immediately after oxidation, the surfaces are negatively charged [72]. Rinsing the channels with a $1 \mathrm{mg} / \mathrm{ml}$ PEI (poly(ethyleneimine)) solution, the positively charged PEI coat the surfaces. In a next step, the channels are coated with a negatively charged $1 \mathrm{mg} / \mathrm{ml}$ PSS (poly(styrene sulfonate)) solution. Finally, the channels are rinsed with a 1mg/ml PLL(20)-g[3.5]-PEG(2) (SurfaceSolutionS GmbH, Zürich, CH) solution, which consists of PLL (poly(L-lysine)) grafted to PEG (poly(ethylene glycol)). PLL is positively charged and bind therefore to the PSS layer. The densely packed PEG chains at the surfaces reduce protein absorption $[73,74]$. The channels are rinsed with water after each coating step. Tests with different coated surfaces showed that less actin bundles stick to the multilayer coated surfaces compared to directly with PLL-g-PEG coated surfaces (without the intermediate step of PEI and PSS). Probably, the binding of PLL to a soft surface is better which is generated by the intermediate coated layers of PEI and PSS.

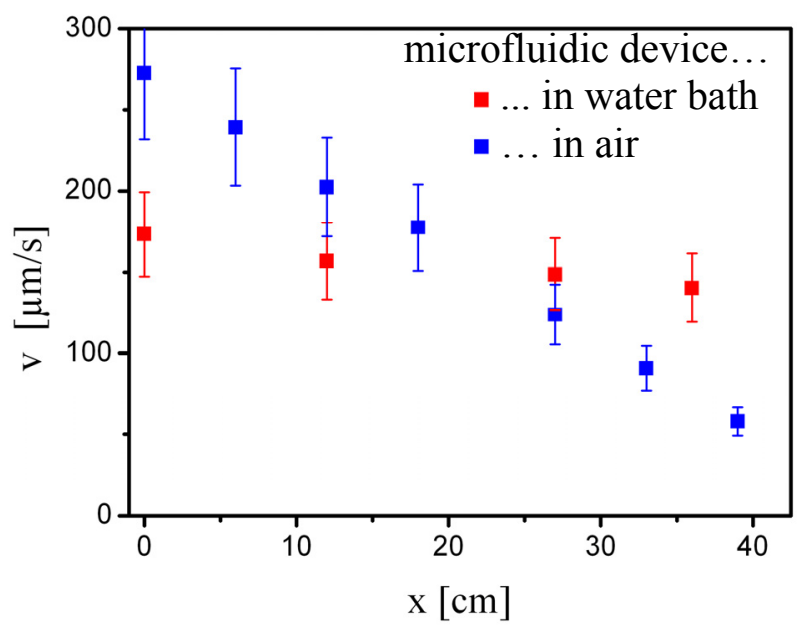

Figure 2-4: Position-dependent velocity inside a microchannel due to diffusion of water through the PDMS. 
During the experiment and also at least three hours before the start of the experiment, the microfluidic device is in a water bath. This is important because PDMS is permeable to water [75]. Water can diffuse (diffusion constant: $D \approx 10^{-9} \mathrm{~m}^{2} / \mathrm{s}$ [75]) through the PDMS out of the channel. A water bath saturates the PDMS with water [76]. Without a water bath, the velocity becomes slower along the channel and the concentration of particles inside the aqueous solution increases. To illustrate this effect, the velocity along a channel (width: $50 \mu \mathrm{m}$, depth: $30 \mu \mathrm{m}$ ) is shown in figure $2-4$. The velocity was firstly measured for a microfluidic device in air and a clear decrease along the channel was found. Having the same microfluidic device for 2 hours in a water bath, the velocity was nearly constant.

\subsection{Liquid Pumping System}

To create a pressure-driven flow inside the channels, we use two different methods. For wide channels $(>20 \mu \mathrm{m} \times 20 \mu \mathrm{m})$ and a high velocities $(>500 \mu \mathrm{m} / \mathrm{s})$, we use custommade syringes pumps. For narrower channels or smaller velocities, the feed of our syringe pumps is too small to ensure a uniform velocity. For these cases, an external pressure is applied at the tubes. During experiments, the pressure is created by the difference of the height ( $\Delta h=1 \mathrm{~cm}-1 \mathrm{~m}$ ) of two reservoirs (see figure 2-5a). To fill the channels and to rinse them with a larger amount of liquid, e.g. for a coating, a higher pressure is needed. In these cases, the pressure is generated by a compressed gas cylinder filled with nitrogen. The gas cylinder is connected by tubes via a reducing valve with a reservoir (see figure 2-5b). A pressure inside the microfluidic device between 0.1 bar and 2 bar can be adjusted by the reducing valve.
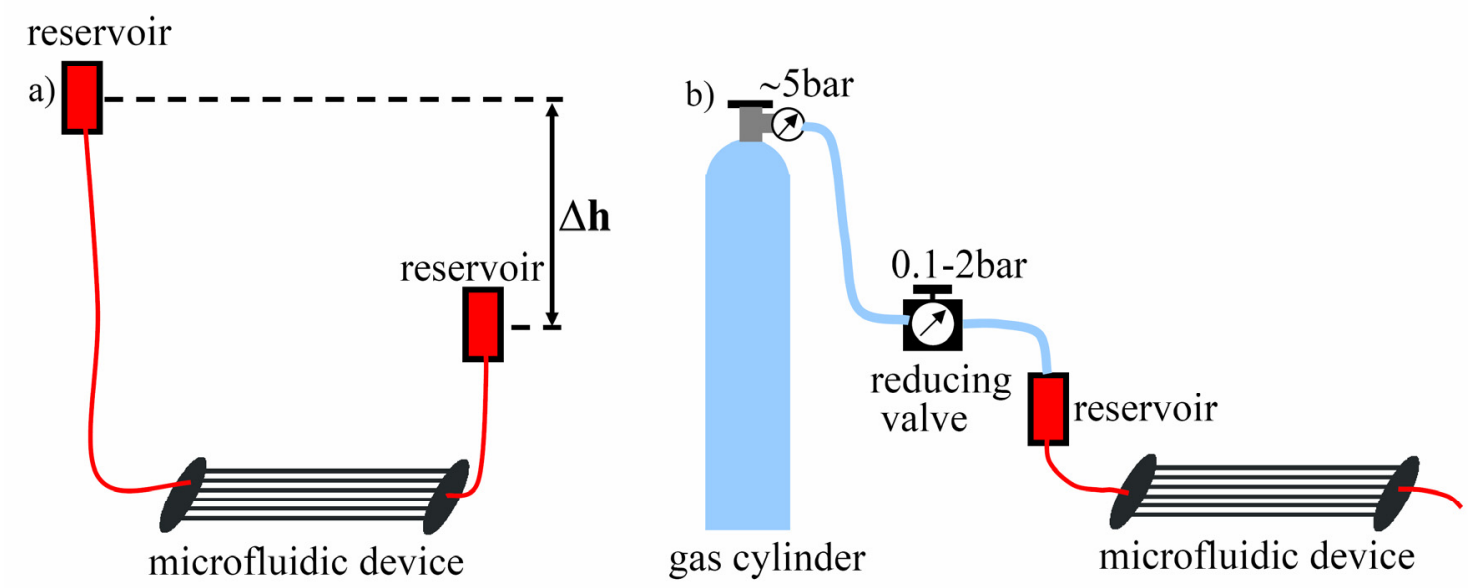

Figure 2-5: Pressure-driven flow: a) by the height of two reservoirs, b) by the pressure of a gas cylinder. 
The flow rate $Q$ within a microchannel is given by $Q=\Delta p / R_{c}$, where $\Delta p$ is the pressure difference across the channel and $R_{c}$ is the channel resistance. The resistance of a circular channel is given by [3]

$$
R_{c}=\frac{8 \eta L_{c}}{\pi r^{4}}
$$

where $\eta$ is the viscosity, $L_{c}$ is the length of the channel and $r$ is the radius. Approximately, this is also the resistance of a symmetric rectangular channel. If the width $d$ of a rectangular channel is much larger than its height $h(d>>h)$, the resistance is given by [3]

$$
R_{c}=\frac{12 \eta L_{c}}{\pi h^{3} d} .
$$

Therefore, a long narrow channel exhibits a higher fluidic resistance than a wide short channel. Our microfluidic devices resist a pressure up to $\sim 2$ bar. To work with low pressures, the resistance of the channels has to be as small as possible. The width and depth of the channels are often specified by experiment, but we try to keep the length of the channels short. Furthermore, we often use parallel channels (see figure 2-5) with total resistance $R_{c}=R_{c, 0} / N_{c}$, where $N_{c}$ is the number of channels and $R_{c, 0}$ is the resistance of a single channel.

\subsection{Fluorescence Microscopy}

The actin filaments, labeled with a fluorescent dye, are imaged by fluorescence microcopy. The used setup is shown in figure 2-6. As a modification of the fluorescence microscope (BX61; Olympus GmbH, Hamburg), a laser (GL532T-300; wavelength: 532nm; power: 300mW; AMS Technologies AG, Martinsried/München) can be used as a light source. This is in particularly important for imaging of fast flowing objects. In figure 2-7, pictures of actin filaments in flow are shown recorded with different exposure times. The contour blurs more and more with increasing exposure time. To avoid this effect, the use of short enough exposure times, depending on the velocity of the actin filaments, is necessary. An increase of the intensity of the light source is a promising possibility to decrease the required exposure time for imaging the actin filaments. Therefore, the experiments of flowing actin filaments are performed with the laser, whereas for non-flowing actin filaments the intensity of the Xe-lamp (75W) is sufficient. For illuminating actin filaments with the laser, an exposure time of only $0.5 \mathrm{~ms}$ is used for imaging. In contrast, an exposure of $25 \mathrm{~ms}$ is required in the case of the Xe-lamp. 


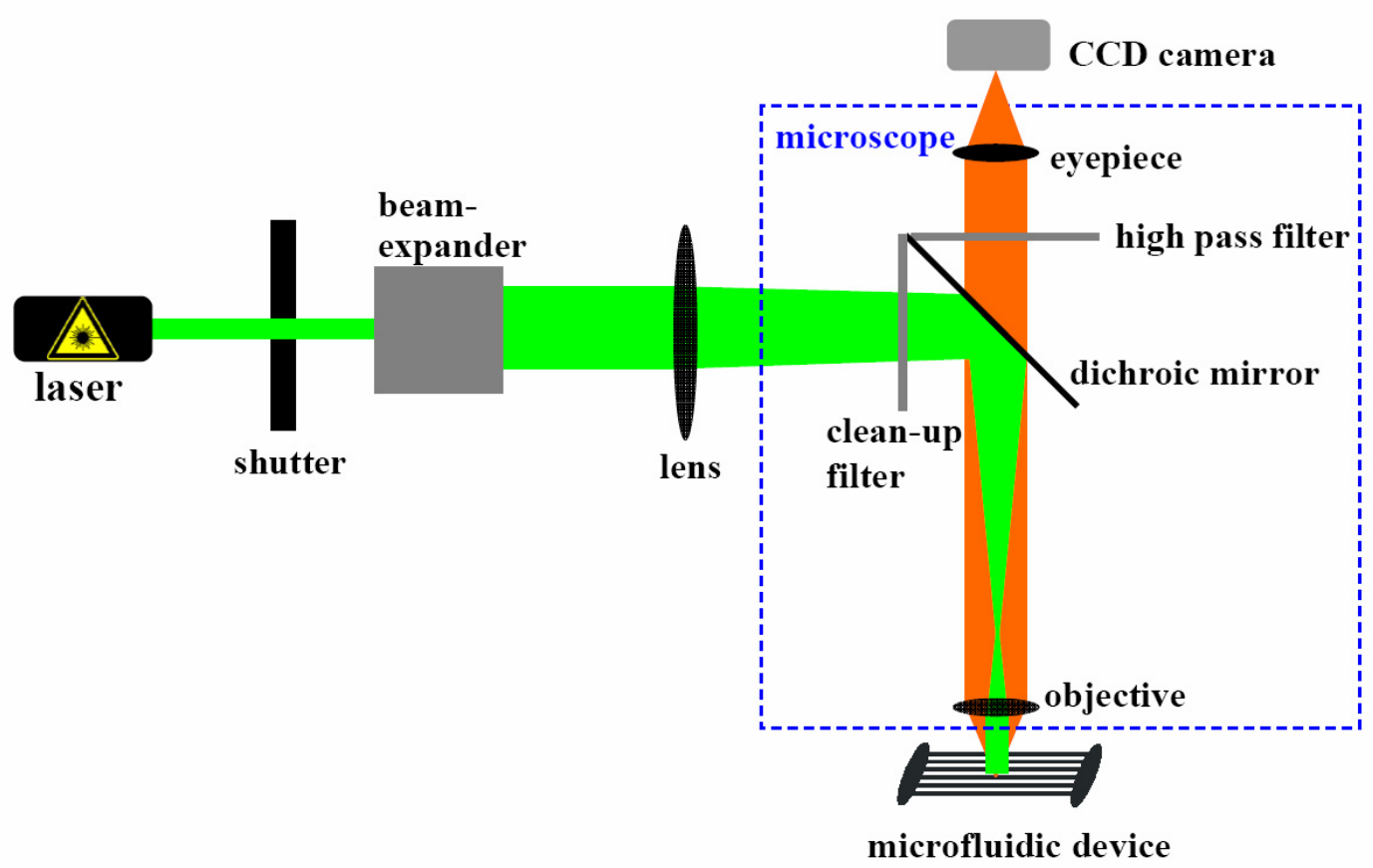

Figure 2-6: Laser setup for fluorescence microscopy.

The sample is illuminated by the laser with an optical path according to Köhler illumination. The parallel laser light (diameter: $\approx 1 \mathrm{~mm}$ ) is expanded 10 times with a beam expander (Thorlabs GmbH, Karlsfeld). With an achromatic lens (focal length: $30 \mathrm{~cm})$, the light is focused to the back focal plane of the objective (100x Plan Apochromat oil immersion) so that parallel laser light illuminates homogenously the sample from above. In doing so, the light passes a laser clean-up filter, and a dichroic mirror reflects the light down to the sample. The light excites dye molecules in the sample and they emit a lower energy light. The used fluorescence dye is rhodamine, and its absorption and emission spectrum is seen in figure 2-8b. The emitted light passes the dichroic mirror and a high pass filter. Wavelengths above $545 \mathrm{~nm}$ are cut off, in particular possible reflections of the laser at the sample. Images are taken with a sensitive CCD-camera (PCO SensiCamQE; PCO, Kehlheim). The transmission spectrum for all filters (AHF, Tübingen) are shown in figure 2-8a.

To avoid photo bleaching and breaking of the actin filaments due to too long illumination of the sample, a shutter creates a stroboscopic illumination. The shutter
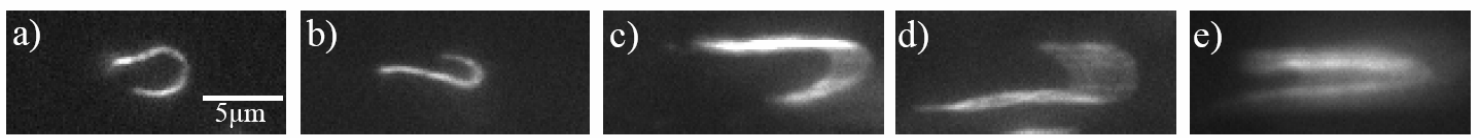

Figure 2-7: Blurring of the contour due to long exposure (velocity of the actin filaments: $\approx 0.7 \mathrm{~mm} / \mathrm{s}$ ).

Exposure times : a) $0.5 \mathrm{~ms}$, b) $1 \mathrm{~ms}$, c) $2.5 \mathrm{~ms}$, d) $5 \mathrm{~ms}$, e) $10 \mathrm{~ms}$. 
a)
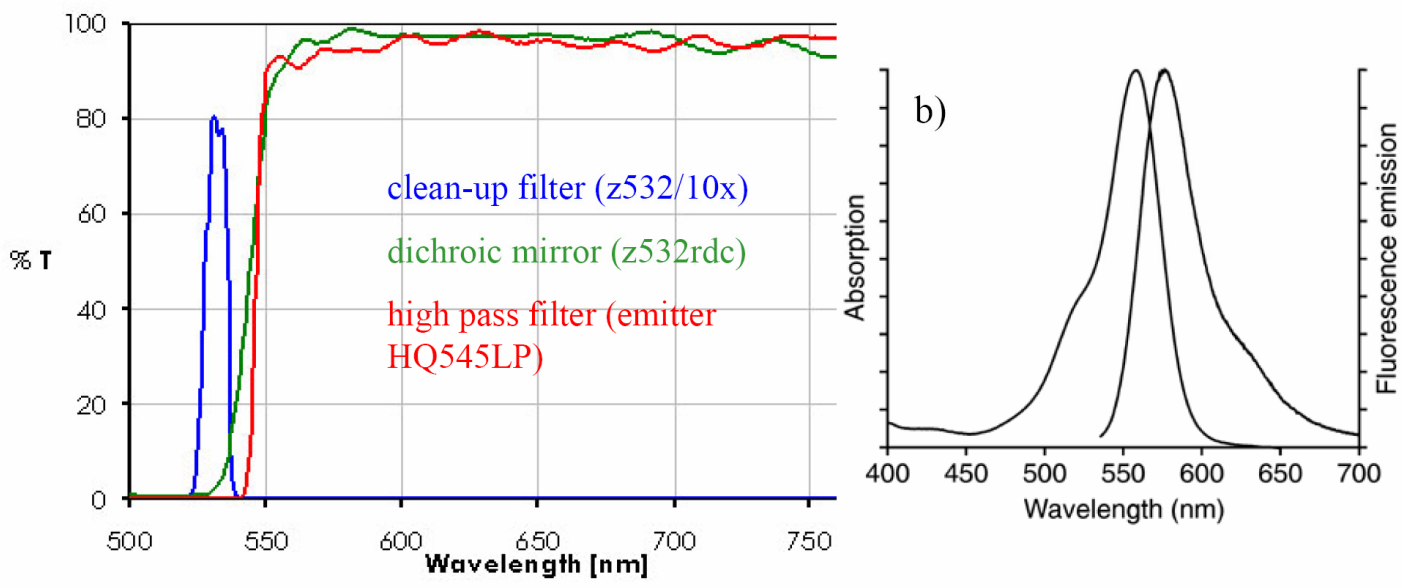

Figure 2-8: a) Transmission spectra of the filter set used for fluorescence microscopy consisting of a clean-up filter, a dichroic mirror, and a high pass filter [77]. b) Absorption and emission spectrum for rhodamine [78].

(LS6; Uniblitz, Rochester, USA) is synchronized with the CCD-camera, so that the camera only takes pictures when the shutter is open. Another advantage of the stroboscopic illumination is the possibility of observing fast changes in the filament contour by illuminating the sample several times during a single image exposure. Consequently, the filament can be observed in shorter time periods than the limitation of the high-sensitive CCD-camera with a frame rate of about $10 \mathrm{~Hz}$ allows. An example of the stroboscopic effect is shown in figure 2-9. The same flowing actin filament is visible in one single image several times.

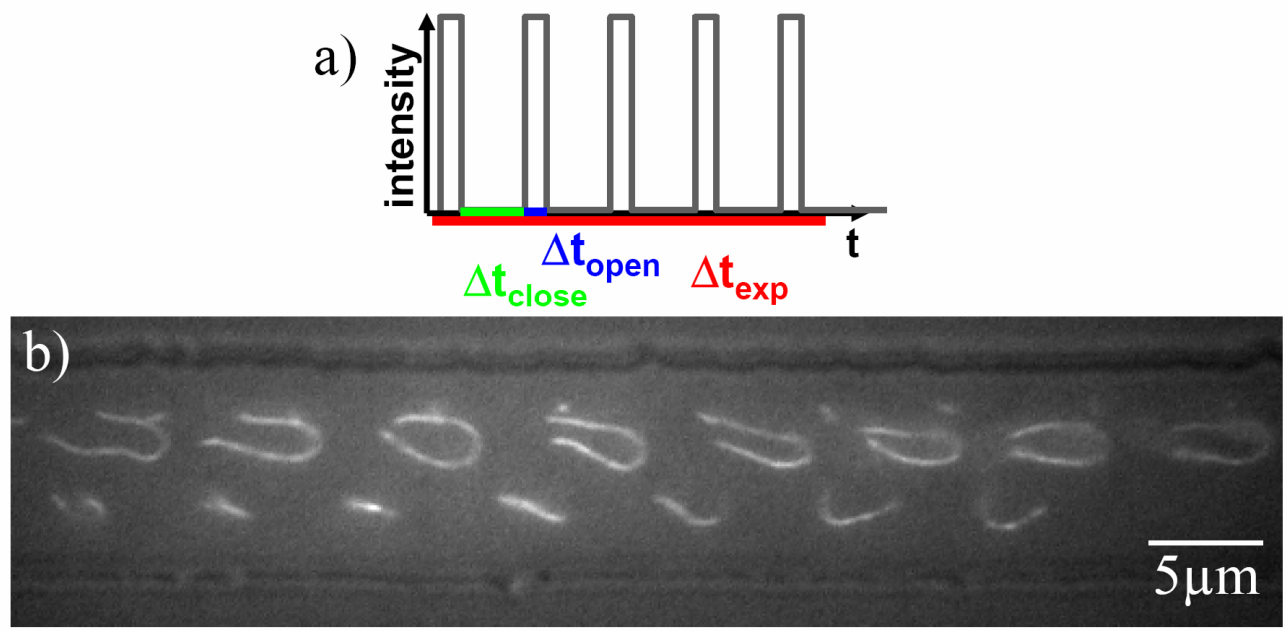

Figure 2-9 a) Stroboscopic illumination ( $\Delta t_{\text {close }}:$ shutter is closed; $t_{\text {open }}$ : shutter is open; $t_{\text {exp }}$ : exposure time of one image). b) Actin filament during a single image exposure: $\Delta t_{\text {open }}=1 \mathrm{~ms}, \Delta t_{\text {close }}=30 \mathrm{~ms}$, $t_{\text {exp }}=300 \mathrm{~ms}$. 


\subsection{Actin Polymerization}

Lyophilized powder of rhodamine labeled actin monomers from rabbit muscle (molecular weight of $43 \mathrm{kDa}$, purity $>99 \%$ ) as well as all buffers are purchased from Cytoskeleton (Denver, USA). After arrival, the actin powder is stored at $-70^{\circ} \mathrm{C}$. The protein is reconstituted to $10 \mathrm{mg} / \mathrm{ml}$ in a buffer solution (containing $5 \mathrm{mM}$ Tris- $\mathrm{HCl}(\mathrm{pH}$ 8.0), $0.2 \mathrm{mM} \mathrm{CaCl}_{2}, 0.2 \mathrm{mM}$ ATP, 5\% sucrose, and 1\% dextran) by dissolving the powder in ultrapure water. The solution is diluted to $0.2 \mathrm{mg} / \mathrm{ml}$ in A-buffer $(5 \mathrm{mM}$ Tris$\mathrm{HCl}$ (pH 8.0), $0.2 \mathrm{mM} \mathrm{CaCl}_{2}, 0.2 \mathrm{mM} \mathrm{ATP}$, and $0.5 \mathrm{mM}$ DTT (dithiothreitol)). The actin concentration of this solution is optimized to obtain few long actin filaments after the polymerization [5]. The solution is placed on ice for two hours to depolymerize actin oligomeres, which can form during storage. To induce actin polymerization, the salt concentration is increased by adding a polymerization buffer $(500 \mathrm{mM} \mathrm{KCl}, 20 \mathrm{mM}$ $\mathrm{MgCl}_{2}$ and $10 \mathrm{mM} \mathrm{ATP}$ ) of $1 / 10$ th of the volume of the actin solution. The critical concentration of monomers above actin polymerize into filaments depends strongly on the conditions of the actin solution like the ion concentration and temperature. At room temperature in absence of monovalent and divalent ions, the critical concentration is greater than $3 \mathrm{mg} / \mathrm{ml}$ [79]. The critical concentration is decreased to $0.03 \mathrm{mg} / \mathrm{ml}$ in the presence of $2 \mathrm{mM} \mathrm{MgCl} 2$ and $50 \mathrm{mM} \mathrm{KCl} \mathrm{[79],} \mathrm{which} \mathrm{are} \mathrm{the} \mathrm{conditions} \mathrm{of} \mathrm{the} \mathrm{actin}$ solution after adding the polymerization buffer. The actin solution is incubated for two hours at room temperature, and the actin monomers polymerize into filaments. Finally, the actin solution is diluted to $70 \mathrm{nM}$ in stabilization buffer $(90 \%$ A-buffer, $10 \%$ polymerization puffer, and $70 \mathrm{nM}$ phalloidin) and stored at $4{ }^{\circ} \mathrm{C}$ until the experiment is started. The actin concentration of $70 \mathrm{nM}$ ensures a dilute solution of actin filaments for single molecule experiments and prevents interactions among each other [5]. Phalloidin stabilizes the actin filaments and prevent them for depolymerization. The mechanical properties of phalloidin-stabilized actin filaments are changed slightly, which should be kept in mind by transferring results of in vitro measurements to in vivo systems. The persistence length is increased by a factor of two [80], but the filaments are still semiflexible and the basic results are valid. 


\section{6 $\alpha$-Actinin}

The ABP $\alpha$-actinin is used for actin bundling experiments. The lyophilized powder (molecular weight about $100 \mathrm{kD}$, purity $>90 \%$ ) is also purchased from Cytoskeleton and stored at $-70^{\circ} \mathrm{C}$. The protein is reconstituted to $2.5 \mathrm{mg} / \mathrm{ml}$ in buffer $(20 \mathrm{mM} \mathrm{NaCl}, 20 \mathrm{mM}$ Tris- $\mathrm{HCl}(\mathrm{pH} 7.2), 5 \%$ sucrose and $1 \%(\mathrm{v} / \mathrm{v})$ dextran). The solution can be stored at $4{ }^{\circ} \mathrm{C}$ several weeks. Before starting an experiment, the solution is diluted to the required concentration. 


\section{Chapter 3}

\section{Worm-Like Chain Model}

\subsection{Persistence Length}

The stiffness of a polymer is often characterized by the persistence length $L_{p}$, which is a measure of the typical length scale for thermal shape fluctuations. The persistence length is given by the ratio of bending rigidity $\kappa$ and thermal energy $k_{b} T$ [81]

$$
L_{p}=\frac{\kappa}{k_{b} T},
$$

where $k_{b}$ is the Boltzmann constant and $T$ is the temperature. Actually, the persistence length depends on temperature, but the persistence length is usually considered at room temperature. For flexible polymers such as $\lambda$-DNA $\left(L_{p} \approx 50 \mathrm{~nm}, L \approx 16.5 \mu \mathrm{m}\right.$ [17]), the persistence length is much smaller than the contour length $L\left(L_{p}<<L\right)$. Conformational entropy dominates the system, and the configuration in equilibrium is coiled $(R<<L ; R$ : end-to-end distance). In the case of stiff polymers, the persistence length is much larger than the contour length $\left(L_{p}>>L\right)$. An example of stiff polymers in biology is microtubules ( $L_{p} \approx 6 \mathrm{~mm}, L \approx 10^{-3}-1 \mathrm{~mm}$ [81]). The contour of stiff polymers is like a rigid $\operatorname{rod}(R \approx L)$, and fluctuations of the contour are hardly visible. For semiflexible polymers, the persistence length and the contour length have the same order of magnitude $\left(L_{p} \approx L\right)$. Actin filaments $\left(L_{p} \approx 13 \mu \mathrm{m}, L \approx 10 \mu \mathrm{m}\right)$, which are described in this thesis, belong to the category of semiflexible polymers. In this case, bending energy dominates the system over conformational entropy. 


\subsection{Worm-Like Chain}

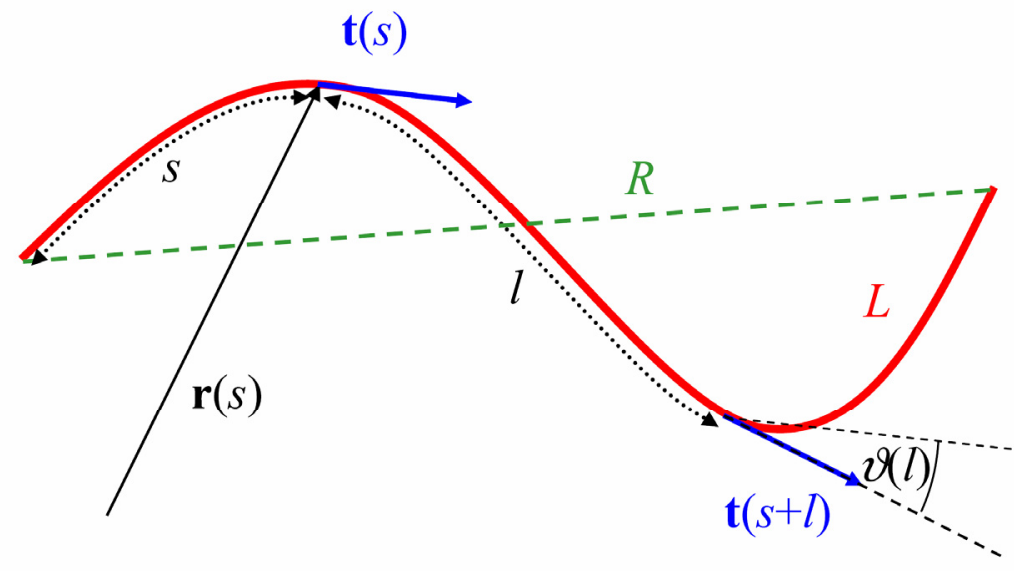

Figure 3-1: Worm-like chain represented by the continuous space curve $r(s)$ parameterized by the arc-length $s$. t $(s)$ : tangent vector, $R$ : end-to-end distance, $L$ : contour length, $\vartheta$ : angle between two tangent vectors.

The polymer model often used to describe semiflexible polymers is the worm-like chain model, which was first introduced by Kratky and Porod [82] in 1949. The polymer is considered as a thin, incompressible, flexible rod with a finite bending rigidity $\kappa$. The polymer is represented by the continuous space curve $\mathbf{r}(s)$, which is parameterized by the arc length $s$ and has a total length of $L$ (see figure 3-1). The statistical properties of the worm-like chain are determined by the free energy functional [83]

$$
\mathcal{H}=\int_{0}^{L} d s \frac{\kappa}{2}\left(\frac{\partial^{2} \mathbf{r}}{\partial s^{2}}\right)^{2} ; \quad\left|\frac{\partial^{2} \mathbf{r}}{\partial s^{2}}\right|=1,
$$

which is obtained by calculating the total elastic energy of a particular conformation. The local inextensibility of the chain is expressed by the constraint $\left|\partial^{2} \mathbf{r} / \partial s^{2}\right|=1$ along the entire contour. The probability for different conformations of the semiflexible polymer is obtained by Boltzmann weighting the energy of the system.

Despite the mathematical difficulties of the model [84], some properties of the semiflexible polymers can be calculated analytically. The correlation of the tangent vectors $\mathbf{t}=\partial \mathbf{r} / \partial s$ shows an exponential decay [83]

$$
\langle\mathbf{t}(s) \cdot \mathbf{t}(s+l)\rangle=\langle\cos \vartheta(l)\rangle=\exp \left(-\frac{l}{L_{p}}\right),
$$

where the brackets denote an average over all possible configurations. If the polymer is confined in two dimensions, equation 3.3 is still correct if a 2-dimensional persistence length $L_{p, 2 \mathrm{D}}=2 L_{p}$ is used. 
The mean square end-to-end distance is given in the worm-like chain model by [83]:

$$
\left\langle R^{2}\right\rangle=2 L_{p}^{2}\left(\exp \left(\frac{L}{L_{p}}\right)-1+\frac{L}{L_{p}}\right) .
$$

Additionally, the end-to-end distance probability distribution can be calculated analytically [85]

$$
G(R / L)=\frac{L_{p}}{L \pi N_{1}} \sum_{k=1}^{\infty} \pi^{2} k^{2}(-1)^{k+1} \exp \left(-\frac{L_{p}}{L} \pi^{2} k^{2}(1-R / L)\right),
$$

where $N_{1}$ is a normalization constant. 


\section{Chapter 4}

\section{Polymers in Microflow}

This chapter considers the behavior of polymers in microflow. In the first section, the flow fields are characterized by the Reynolds number. Then, the forces acting on a Brownian particle are described. Finally, the behavior of polymers in shear flow and migration effects in non-homogenous flow are illustrated by a dumbbell model.

\subsection{Reynolds Number}

Flow of a fluid is often classified into two flow regimes: the turbulent flow and the laminar flow [86]. Turbulent flow is chaotic and unpredictable in contrast to laminar flow, where the velocity field is either stationary or slowly varying in time. Neighboring streamlines flow parallel to each other in the case of laminar flow, whereas streams are mixed chaotically in turbulent flow. The dimensionless Reynolds number Re indicates whether the flow is laminar or turbulent, and is defined as [86]

$$
\operatorname{Re}=\frac{d \rho v}{\eta}
$$

where $d$ is a characteristic length scale of the system (e.g. channel width), $\rho$ is the density, $\eta$ is the viscosity and $v$ is the velocity of the fluid. The Reynolds number is a measure of the ratio of inertial and viscous forces on the fluid. For small Reynolds numbers $(R e<1)$, viscous forces dominate and the flow is laminar. In an intermediate regime $1<R e<R e_{c r i t}$, where the critical Reynolds number $R e_{c r i t}$ is typically 1000-2500 depending on the geometry [87], the flow is still laminar but inertial forces become significant. Above the critical Reynolds number $\left(R e>R e_{\text {crit }}\right)$, the flow is turbulent. In microfluidics, the Reynolds numbers are usually very small, the flow is laminar, and inertial effects are irrelevant. For water ( $\rho \approx 1 \mathrm{~kg} / \mathrm{L}, \eta \approx 1 \mathrm{mPa} \cdot \mathrm{s})$ flowing with a velocity 
of $1 \mathrm{~mm} / \mathrm{s}$ in a straight channel with a width of $10 \mu \mathrm{m}$ (which is used in the experiments), the Reynolds number is $R e \approx 10^{-2}$.

\subsection{Motion of Brownian Particles}

\subsubsection{Drag Force}

A particle moving through a viscous fluid will experience a frictional force from the surrounding solvent molecules that oppose its motion. This drag force in the regime of small Reynolds numbers is proportional to the velocity [88]:

$$
\mathbf{F}_{\text {drag }}=-c_{f} \mathbf{v},
$$

where $c_{f}$ is the drag coefficient and $\mathbf{v}$ is the velocity of the particle relative to the fluid velocity.

For a sphere, the drag coefficient is [81]

$$
c_{f}=6 \pi \eta r,
$$

where $r$ is the radius of the sphere.
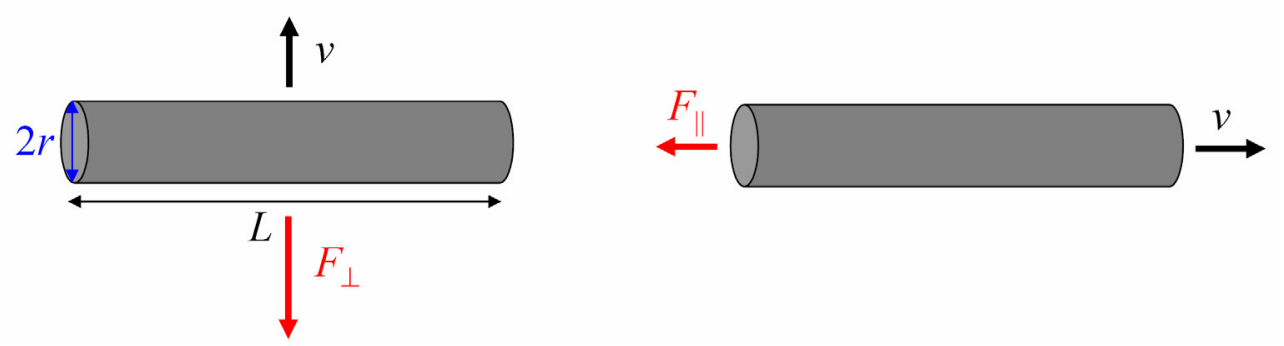

Figure 4-1: Drag force of a cylinder moving with velocity $v$. The drag force $F \perp$ for a motion perpendicular to the axis of the cylinder is larger than the drag force $F_{\|}$in direction to the axis.

For a cylinder with length $L$ and radius $r$ (see figure 4-1), the direction of the movement is important. The drag coefficient for a motion perpendicular to the axis of the cylinder is [81]

$$
c_{\perp} \approx \frac{4 \pi \eta L}{\ln (L / 2 r)+0.84},
$$

which is larger than the drag coefficient of a motion parallel to the axis [81]

$$
c_{\|} \approx \frac{2 \pi \eta L}{\ln (L / 2 r)-0.2} .
$$




\subsubsection{Diffusion}

When a small particle is suspended in a fluid, it is subject to collisions with the surrounding molecules of the fluid caused by thermal energy of the system. During each collision, the change in momentum imparted on the particle varies randomly, which makes it undergo a "random-walk" motion, called Brownian motion. Diffusion is the spreading of free particles due to their Brownian motion and can be characterized by the mean square displacement [89]

$$
\left\langle\mathbf{r}^{2}\right\rangle=2 n_{d} D t
$$

in a time $t$, where $D$ is the diffusion coefficient of the particles in the surrounding fluid, and $n_{d}$ is the dimension of the system.

To describe the molecular Brownian motion of a free particle, the forces acting on it should be considered. First of all, the random collisions of the solvent molecules with the Brownian particle result in a rapidly varying force $\mathbf{F}_{\text {brown }}(t)$. This force fluctuates on the time scale of $10^{-14} \mathrm{~s}$ [89]. As soon as the particle attains a finite velocity, it feels a drag force (see equation 4.2) due to systematic collisions of the particle with the solvent molecules. Newton's equation of motion for a Brownian particle with mass $m$ can be written as [90]

$$
m \frac{\partial \mathbf{v}}{\partial t}=-c_{f} \mathbf{v}+\mathbf{F}_{\text {brown }}(t) .
$$

This equation is also referred to as the Langevin equation. The fluctuations of the random force $\mathbf{F}_{\text {brown }}(\mathrm{t})$ can be characterized in thermodynamic equilibrium by the fluctuation dissipation theorem [90]:

$$
\begin{aligned}
\left\langle\mathbf{F}_{\text {brown }}(t)\right\rangle & =0 \\
\left\langle\mathbf{F}_{\text {brown }}(t) \mathbf{F}_{\text {brown }}\left(t^{\prime}\right)\right\rangle & =2 k_{b} T c_{f} \delta\left(t-t^{\prime}\right) .
\end{aligned}
$$

The brackets denote ensemble averaging with respect to many possible realizations of the fluctuating force $\mathbf{F}_{\text {brown }}, k_{b}$ is the Boltzmann constant, $T$ is the temperature, and $\delta(t)$ is the delta distribution.

Neglecting inertial forces (small Reynolds numbers), integration of the Langevin equation 4.7 gives the position $\mathbf{r}(t)$ of a particle at time $t$ with velocity $\mathbf{v}(t)=\mathrm{d} \mathbf{r}(t) / \mathrm{d} t$. For a one-dimensional motion of a particle, the position at time $t$ is given by

$$
x(t)=x(0)+\frac{1}{c_{f}} \int_{0}^{t} F_{\text {brown }}\left(t^{\prime}\right) d t^{\prime} .
$$


The mean square displacement can be calculated as

$$
\langle(x(t)-x(0))(x(t)-x(0))\rangle=\frac{1}{c_{f}^{2}}\left\langle\int_{0}^{t} d t_{1} F_{\text {brown }}\left(t_{1}\right) \int_{0}^{t} d t_{2} F_{\text {brown }}\left(t_{2}\right)\right\rangle,
$$

which can be simplified to

$$
\langle(x(t)-x(0))(x(t)-x(0))\rangle=\frac{1}{c_{f}^{2}} \int_{0}^{t} \int_{0}^{t} d t_{1} d t_{2}\left\langle F_{\text {brown }}\left(t_{1}\right) F_{\text {brown }}\left(t_{2}\right)\right\rangle .
$$

With the fluctuation dissipation theorem (equation 4.8), the mean square displacement results in

$$
\langle(x(t)-x(0))(x(t)-x(0))\rangle=\frac{2 k_{b} T}{c_{f}} t .
$$

Comparison with equation 4.6 gives the Einstein-Stokes relation between the diffusion coefficient $D$ and the drag coefficient $c_{f}$.

$$
D=\frac{k_{b} T}{c_{f}}
$$

\subsubsection{Hydrodynamic Interactions}

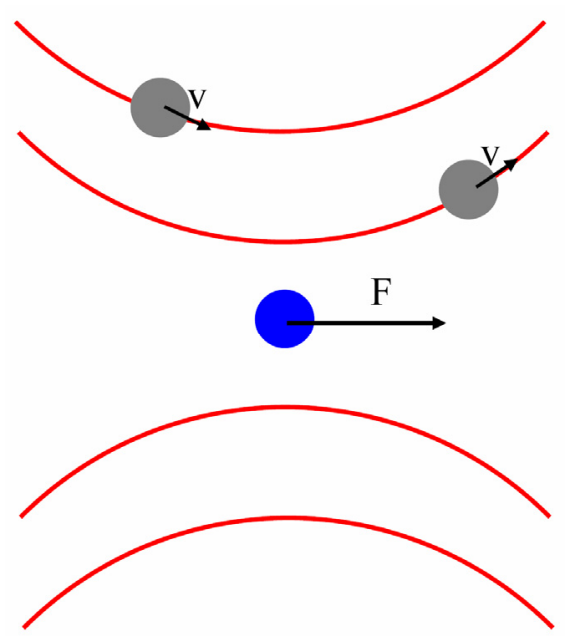

Figure 4-2: Illustration of hydrodynamic interactions. One particle (blue) is moving through a solvent due to a force $F$. This motion excites a flow field (red lines). Other particles (grey) feel this flow field and move along its streamlines.

Several particles suspended in a fluid are coupled by the displacement of the molecules of that fluid. The motion of one particle excites a motion of the surrounding solvent molecules, and the resultant flow field is felt by all other particles and influences their 
motions. Hence, the particles experience a force whose origin is the movement of another particle. This coupling is called a hydrodynamic interaction. In figure 4-2, such a situation is illustrated.

In general, all particles moving with a velocity $\mathrm{d} \mathbf{r} / \mathrm{d} t \neq 0$ relative to the fluid perturb the velocity field $\mathbf{v}(\mathbf{r})$ of the solvent. If the drag force $\mathbf{F}_{d r a g, j}$, which acts on the $j$-th particle at the position $\mathbf{r}_{j}$, is weak enough, the perturbation of the velocity field is small. Then, the perturbation $\mathbf{v}^{\prime}\left(\mathbf{r}_{i}\right)$ of the solvent velocity at position $\mathbf{r}_{i}$ of the $i$-th particle can be approximated by a linear function of the hydrodynamic drag force $\mathbf{F}_{\text {drag, } j}$ [89]. Including the motion of all particles, the perturbation flow is given by

$$
\mathbf{v}^{\prime}\left(\mathbf{r}_{i}\right)=\sum_{j} \mathbf{\Omega}_{i j} \mathbf{F}_{\text {drag }, j},
$$

where $\Omega$ is the hydrodynamic interaction tensor, which is for $N$ particles a $N$ x $N$ tensor, whose components $\boldsymbol{\Omega}_{i j}$ are a $3 \times 3$ matrix. For free particles, the components of the hydrodynamic interaction tensor $\Omega_{i j}$ are a function of the displacement $\left(\mathbf{r}_{i}-\mathbf{r}_{j}\right)$ between the particles $i$ and $j$ [89]. The velocity of the solvent can be estimated by a linear superposition

$$
\mathbf{v}(\mathbf{r})+\mathbf{v}^{\prime}\left(\mathbf{r}_{i}\right)
$$

of the unperturbed velocity $\mathbf{v}(\mathbf{r})$ of the fluid and the perturbation $\mathbf{v}^{\prime}(\mathbf{r})$ due to hydrodynamic interactions.

For a polymer, the hydrodynamic interactions of the different segments depend on the polymer conformation and decrease slowly with distance between the interacting parts. Hydrodynamic interactions become very important if the length scale of the system is comparable with a characteristic length scale of the polymer [90].

\subsection{Polymers in Shear Flow}

\subsubsection{Weissenberg Number}

In addition to the forces (drag force, Brownian force, and hydrodynamic interactions) acting on a rigid particle in a fluid considered in section 4.2, elastic forces are important for a polymer molecule suspended in a solvent [90]. The shear rate of a flow is a measure of the deformation rate of a fluid and is in general described by a tensor [88]. For a planar flow with velocity field $\boldsymbol{v}=v_{x} \mathbf{e}_{\mathbf{x}}+v_{y} \mathbf{e}_{\mathbf{y}}$, the shear rate is given by [91]

$$
\dot{\gamma}=\frac{\partial v_{x}}{\partial y}+\frac{\partial v_{y}}{\partial x}
$$


The flow can deform the polymer depending on the shear rate and the elasticity of the polymer. The dimensionless Weissenberg number [8]

$$
W i=\dot{\gamma} \tau_{r e l}
$$

relates a characteristic relaxation time $\tau_{\text {rel }}$ of the system to the flow deformation time $\dot{\gamma}^{-1}$. For small Weissenberg numbers $(W i<<1)$, the polymer relaxes before the flow deforms it significantly. When the Weissenberg number becomes $\sim 1$, the polymer has no time to relax back in its equilibrium state and polymer deformations become significant [8].

\subsubsection{Dumbbell Model}

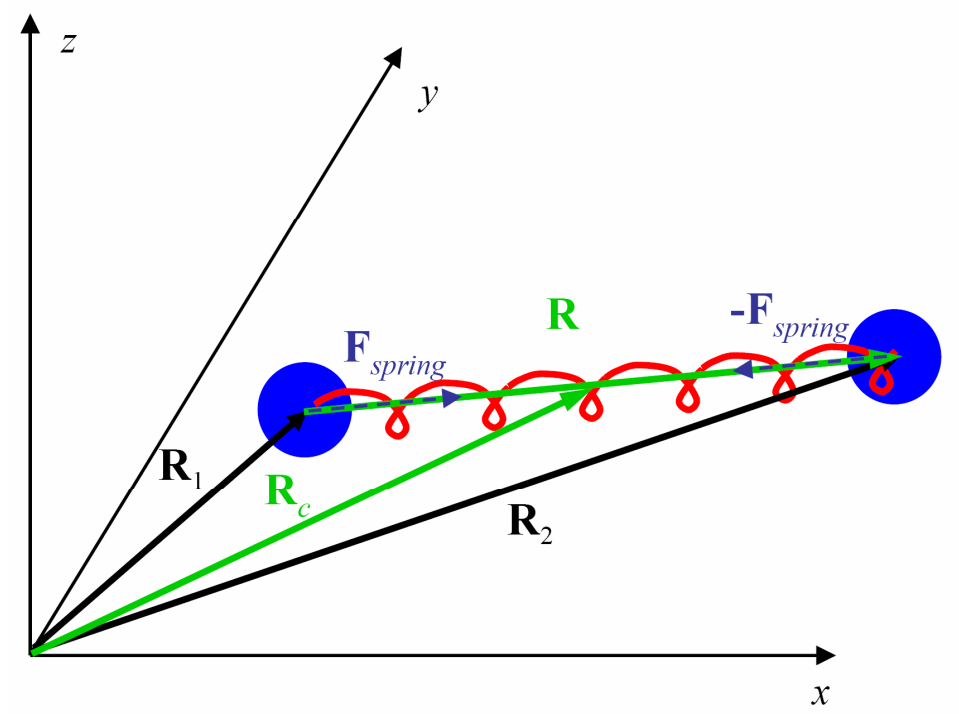

Figure 4-3: Dumbbell model. The dumbbell consists of two beads which are connected by a spring. $F_{\text {spring }}$ is the spring force, $R_{1}$ and $R_{2}$ are the positions of the beads, $R$ is the end-to-end vector and $R_{c}$ is the center-of-mass vector.

A dumbbell model can be used as a simple model for a polymer [90, 92, 93]. The dumbbell consists of two beads of radius $a$ and negligible mass, which are connected by a spring (see figure 4-3). The beads represent the ends of the polymer, while the spring represents entropic forces. The dumbbell can be characterized by the positions of the beads $\mathbf{R}_{1}$ and $\mathbf{R}_{2}$ or by its end-to-end vector $\mathbf{R}=\mathbf{R}_{2}-\mathbf{R}_{1}$ and its center-of-mass vector $\mathbf{R}_{\mathrm{c}}=\left(\mathbf{R}_{2}+\mathbf{R}_{1}\right) / 2$. The center-of-mass vector $\mathbf{R}_{c}$ describes the transport of a dumbbell in a flow field and the end-to-end vector $\mathbf{R}$ describes the conformation (stretching and orientation) of the dumbbell. The extension of the dumbbell is given by $R=|\mathbf{R}|$. 
The positions of the beads $\mathbf{R}_{1}$ and $\mathbf{R}_{2}$ are determined by hydrodynamic drag forces, Brownian forces, hydrodynamic interactions, and the elastic force of the spring. The force balance at the $i$-th bead is given by

$$
\mathbf{F}_{\text {drag }, i}+\mathbf{F}_{\text {brown }, i}+\mathbf{F}_{H I, i}+\mathbf{F}_{\text {spring }, i}=0 .
$$

Inserting the hydrodynamic drag force (equation 4.2) and describing the flow field at the position $\mathbf{R}_{i}$ of the $i$-th bead by a superposition (see equation 4.15 ) of the (unperturbed) solvent velocity $\mathbf{v}\left(\mathbf{R}_{i}\right)$ and the perturbation $\mathbf{v}^{\prime}\left(\mathbf{R}_{i}\right)$ due to hydrodynamic interactions, the Langevin equation for the $i$-th bead of a dumbbell results in

$$
\frac{\mathrm{d} \mathbf{R}_{i}}{\mathrm{~d} t}=\mathbf{v}\left(\mathbf{R}_{i}\right)+\mathbf{v}^{\prime}\left(\mathbf{R}_{i}\right)+\frac{1}{c_{f}} \mathbf{F}_{\text {brown }, i}(t)+\frac{1}{c_{f}} \mathbf{F}_{\text {spring }, i},
$$

where $\mathrm{d} \mathbf{R}_{i} / \mathrm{d} t$ is the velocity of the $i$-th bead.

In linear approximation of the perturbation velocity (see equation 4.14), the Langevin equation can be written as

$$
\begin{aligned}
\frac{\mathrm{d} \mathbf{R}_{i}}{\mathrm{~d} t} & =\mathbf{v}\left(\mathbf{R}_{i}\right)+\sum_{j \neq i} \boldsymbol{\Omega}_{i j}\left(\mathbf{F}_{\text {brown }, j}(t)+\mathbf{F}_{\text {spring, }, j}\right)+\frac{1}{c_{f}}\left(\mathbf{F}_{\text {brown }, i}(t)+\mathbf{F}_{\text {spring }, i}\right) \\
& =\mathbf{v}\left(\mathbf{R}_{i}\right)+\frac{1}{k_{B} T} \sum_{j=1}^{2} \mathbf{D}_{i j}\left(\mathbf{F}_{\text {brown }, j}(t)+\mathbf{F}_{\text {spring }, j}\right),
\end{aligned}
$$

where $\mathbf{D}$ is the diffusion tensor, whose components are defined as

$$
\mathbf{D}_{i j}=k_{B} T\left(\frac{1}{c_{f}} \delta_{i j} \mathbf{I}+\mathbf{\Omega}_{i j}\right),
$$

$\delta_{\mathrm{ij}}$ is the Kronecker symbol, which is one for $i=j$ and otherwise zero, and $\mathbf{I}$ is the unit matrix.

With equation 4.20, the evolution of the end-to-end vector

$$
\begin{aligned}
\frac{\mathrm{d} \mathbf{R}}{\mathrm{d} t} & =\frac{\mathrm{d} \mathbf{R}_{\mathbf{2}}}{\mathrm{d} t}-\frac{\mathrm{d} \mathbf{R}_{\mathbf{1}}}{\mathrm{d} t} \\
& =\left(\mathbf{v}\left(\mathbf{R}_{2}\right)-\mathbf{v}\left(\mathbf{R}_{1}\right)\right)+\frac{1}{k_{b} T} \sum_{j=1}^{2}\left(\mathbf{D}_{2 j}-\mathbf{D}_{1 j}\right)\left(\mathbf{F}_{\text {spring }, j}+\mathbf{F}_{\text {brown }, j}(t)\right)
\end{aligned}
$$

and the evolution of the center-of-mass vector

$$
\begin{aligned}
\frac{\mathrm{d} \mathbf{R}_{c}}{\mathrm{~d} t} & =\frac{1}{2}\left(\frac{\mathrm{d} \mathbf{R}_{2}}{\mathrm{~d} t}+\frac{\mathrm{d} \mathbf{R}_{1}}{\mathrm{~d} t}\right) \\
& =\frac{1}{2} \sum_{i=1}^{2} \mathbf{v}\left(\mathbf{R}_{i}\right)+\frac{1}{2 k_{b} T} \sum_{i=1}^{2} \sum_{j=1}^{2} \mathbf{D}_{i j}\left(\mathbf{F}_{\text {spring }, j}+\mathbf{F}_{\text {brown }, j}(t)\right)
\end{aligned}
$$

can be derived.

The spring force acting on the first bead $\mathbf{F}_{\text {spring, } 1}$ is opposite to the spring force $\mathbf{F}_{\text {spring, } 2}$ 
acting on the second bead:

$$
\mathbf{F}_{\text {spring }, 1}=-\mathbf{F}_{\text {spring }, 2} \equiv \mathbf{F}_{\text {spring }} \text {. }
$$

The simplest form for the spring force is the Hookian law [90]

$$
\mathbf{F}_{\text {spring, Hooke }}=H \mathbf{R} \text {, }
$$

where $H$ is the spring constant. However, a Hookian dumbbell can be infinitely expanded, which is unphysical. A more realistic model is a FENE (finitely extensible non-linear elastic) dumbbell, which incorporates nonlinear effects of stretching a polymer into the model. The spring force can be approximated by the Warner spring law [90]:

$$
\mathbf{F}_{\text {spring }, F E N E}=\frac{H}{1-(R / L)^{2}} \mathbf{R},
$$

where $L$ is the maximal extension of the dumbbell, corresponding to the contour length of the polymer. The relaxation time in the FENE dumbbell model is related to the spring constant by [90]

$$
\tau_{r e l}=\frac{c_{f}}{4 H}
$$

and the extension of the dumbbell without external force by [90]

$$
R_{e q}=\frac{k_{b} T}{H} .
$$

For the case that hydrodynamic interactions can be neglected, the evolution of the endto-end vector (equation 4.22) can be simplified to

$$
\frac{\mathrm{d} \mathbf{R}}{\mathrm{d} t}=\left(\mathbf{v}\left(\mathbf{R}_{2}\right)-\mathbf{v}\left(\mathbf{R}_{1}\right)\right)+\frac{2}{c_{f}} \mathbf{F}_{\text {spring }}+\boldsymbol{\xi}_{\text {brown }}(t),
$$

where $\xi_{\text {brown }}(t)$ describes the thermal fluctuations of the entire dumbbell. The thermal noise $\xi_{\text {brown }}(t)$ of the dumbbell can be modeled by a Gaussian white noise, which is characterized by the correlation [94]

$$
\begin{aligned}
& \left\langle\boldsymbol{\xi}_{\text {brown }}(t)\right\rangle=0 \\
& \left\langle\boldsymbol{\xi}_{\text {brown }}(t) \boldsymbol{\xi}_{\text {brown }}\left(t^{\prime}\right)\right\rangle=\frac{R_{e q}{ }^{2}}{\tau_{\text {rel }}} \boldsymbol{\delta}\left(t-t^{\prime}\right) .
\end{aligned}
$$

With the Warner spring law (equation 4.26), the end-to-end vector is determined by

$$
\frac{\mathrm{d} \mathbf{R}}{\mathrm{d} t}=\mathbf{v}\left(\mathbf{R}_{2}\right)-\mathbf{v}\left(\mathbf{R}_{1}\right)-\frac{1}{2 \tau_{r e l}\left(1-(R / L)^{2}\right)} \mathbf{R}+\boldsymbol{\xi}_{\text {brown }}(t) .
$$

The velocity field is smooth on the scale of the typical size of a polymer in laminar flow [89]. The velocity difference $\mathbf{v}\left(\mathbf{R}_{1}\right)-\mathbf{v}\left(\mathbf{R}_{2}\right)$ is approximately proportional to the velocity 
gradient, such that the $k$-th component of the velocity difference can be estimated as

$$
\begin{aligned}
v_{k}\left(\mathbf{R}_{2}\right)-v_{k}\left(\mathbf{R}_{1}\right) & =\frac{\partial v_{k}\left(\mathbf{R}_{c}\right)}{\partial x} R_{x}+\frac{\partial v_{k}\left(\mathbf{R}_{c}\right)}{\partial y} R_{y}+\frac{\partial v_{k}\left(\mathbf{R}_{c}\right)}{\partial z} R_{z}+O\left(\mathbf{R}^{2}\right) \\
& =\sum_{j=1}^{3} \nabla_{j} v_{k}\left(\mathbf{R}_{c}\right) R_{j}+O\left(\mathbf{R}^{2}\right),
\end{aligned}
$$

where $\mathbf{v}\left(\mathbf{R}_{c}\right)$ is the velocity at the position of the center-of-mass of the dumbbell, and $R_{x}$, $R_{y}, R_{z}$ are the components of the end-to-end vector $\mathbf{R}$. With this approximation, the $k$-th component of end-to-end vector (equation 4.31) is determined by

$$
\frac{\mathrm{d} R_{k}}{\mathrm{~d} t}=\sum_{j} \nabla_{j} v_{k}\left(\mathbf{R}_{c}\right) R_{j}-\frac{1}{2 \tau_{r e l}\left(1-(R / L)^{2}\right)} R_{k}+\xi_{\text {brown }, k}(t) .
$$

\subsubsection{Dumbbell in Simple Shear Flow}

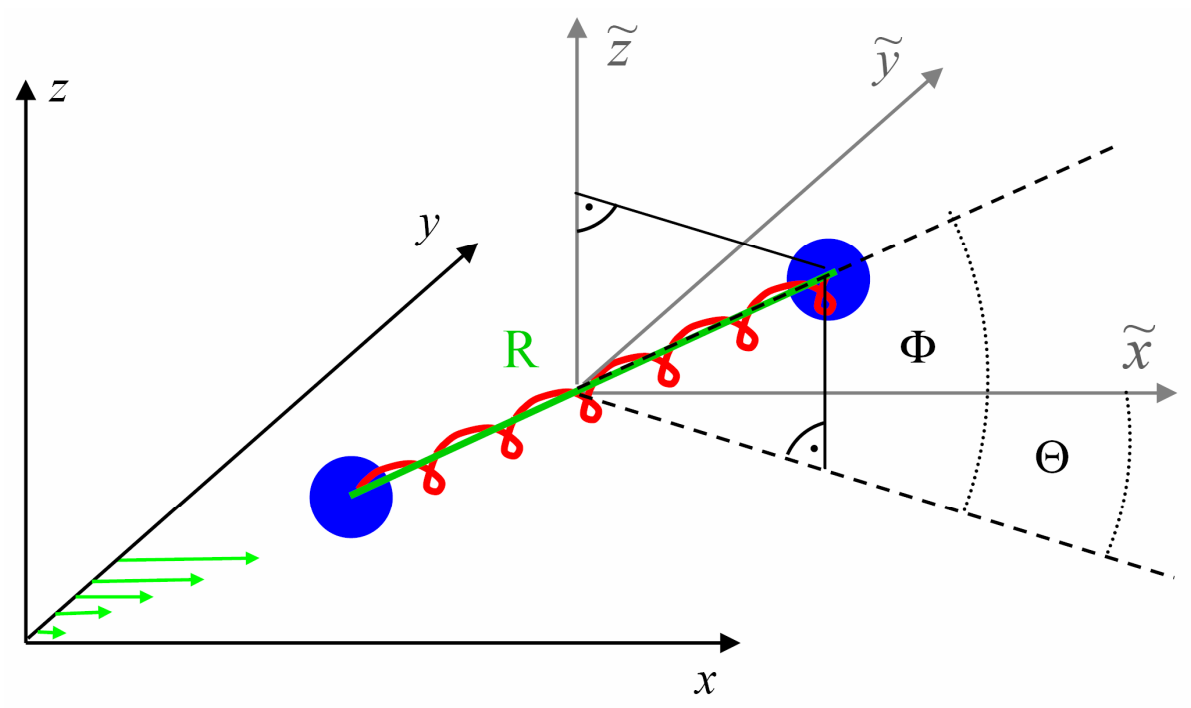

Figure 4-4: Dumbbell in simple shear flow. The coordinate system $(\tilde{\mathbf{x}}, \tilde{\mathbf{y}}, \tilde{\mathbf{z}})$ is moving with the center-of-mass velocity of the dumbbell. Orientation of the dumbbell can be described by the angles $\Theta$ and $\Phi$ in a spherical coordinate system $(R, \Theta, \Phi)$.

A simple shear flow is described by the velocity field [89]

$$
\mathbf{v}=\left(\begin{array}{c}
\dot{\gamma} y \\
0 \\
0
\end{array}\right),
$$

where $\dot{\gamma}$ is the shear rate (see equation 4.16). 
A simple shear flow has two effects on the dumbbell: advection of the dumbbell due to the velocity of the surrounding solvent, and rotation and stretching of the dumbbell because of velocity gradients. Rotation and stretching of the dumbbell are described by the end-to-end vector, and advection by the motion of the center-of-mass vector. The center-of-mass vector primarily follows the streamlines of the fluid. Diffusion of the center-of-mass away from the present streamline is small and occurs at larger time scales than the rotation and stretching of the dumbbell. Therefore, the equation of motion of the end-to-end vector can be decoupled from the equation of motion of the center-of-mass vector and in the following discussions the situation is always considered in a moving coordinate system $(\tilde{x}, \tilde{y}, \widetilde{z})$ with the center-of-mass as its origin (see figure 4-5).

With the known velocity field (equation 4.34) for simple shear flow, the evolution of the $k$-th component of the end-to-end vector (equation 4.33) can be written as [94]

$$
\frac{\mathrm{d} R_{k}}{\mathrm{~d} t}=\dot{\gamma} \delta_{k \widetilde{x}} R_{\widetilde{y}}-\frac{1}{2 \tau_{r e l}\left(1-R^{2} / L^{2}\right)} R_{k}+\xi_{\text {brown }, k}(t) .
$$

To investigate the orientation dynamics, it is useful to parameterize the end-to-end vector $\mathbf{R}$ into spherical coordinates $(R, \Theta, \Phi)$ and the evolution of the end-to-end vector $\mathbf{R}=(R \cos \Phi \cos \Theta, R \cos \Phi \sin \Theta, R \sin \Theta)$ can be transformed to [95]:

$$
\begin{gathered}
\partial_{t} \Theta=-\dot{\gamma} \sin ^{2} \Theta+\xi_{\Theta, \text { brown }}(t) \\
\partial_{t} \Phi=-\dot{\gamma} \sin \Theta \cos \Theta \sin \Phi \cos \Phi+\xi_{\Phi, \text { brown }}(t) \\
\partial_{t} \ln R=-\frac{1}{2 \tau_{\text {rel }}\left(1-R^{2} / L^{2}\right)}+\dot{\gamma} \sin \Theta \cos \Theta \cos ^{2} \Phi+\xi_{R, \text { brown }}(t) .
\end{gathered}
$$

According to 4.36 and 4.37 , the probability distribution function $P(\Theta, \Phi)$ for the angles is symmetric with respect to $\Phi$ but not symmetric with respect to $\Theta$. Therefore, the averaged value of $\Theta$ is not zero. The angle $\Theta_{t}$ of the maximum of the probability distribution and the half-width $\Delta \Theta$ can be estimated by balancing the shear and noise terms in equation 4.36 , where the noise $\xi_{\Theta, \text { brown }}(t)$ can be described as Gaussian white noise with correlation [94]

$$
\left\langle\xi_{\Theta, \text { brown }}(t) \xi_{\Theta, \text { brown }}\left(t^{\prime}\right)\right\rangle=\frac{R_{e q}{ }^{2}}{R^{2} \tau_{r e l}} \delta\left(t-t^{\prime}\right) .
$$

This leads to scaling laws at large Weissenberg numbers $W i>>1$ (or $\Theta<<1$ ) [94]:

$$
\Delta \Theta \propto \Theta_{t} \propto W i^{-1 / 3}\left(\frac{R_{e q}}{R}\right)^{2 / 3} .
$$


In the regime $R<L$, the extension of a flexible polymer can be approximately described by a linear elastic dumbbell $R \propto W i$. This leads to the scaling [94]

$$
\Delta \Theta \propto \Theta_{t} \propto W i^{-1} .
$$

If flexible polymers are strongly elongated or stiff polymers are considered, the polymers can be treated as a rigid $\operatorname{rod}(R \approx L)$, and a quite different scaling behavior [94]

$$
\Delta \Theta \propto \Theta_{t} \propto W i^{-1 / 3}
$$

is found.

These scaling laws have been also confirmed by a more realistic Gaussian semiflexible polymer model [96] and by numerical simulations [94].

\subsection{Cross-Streamline Migration}

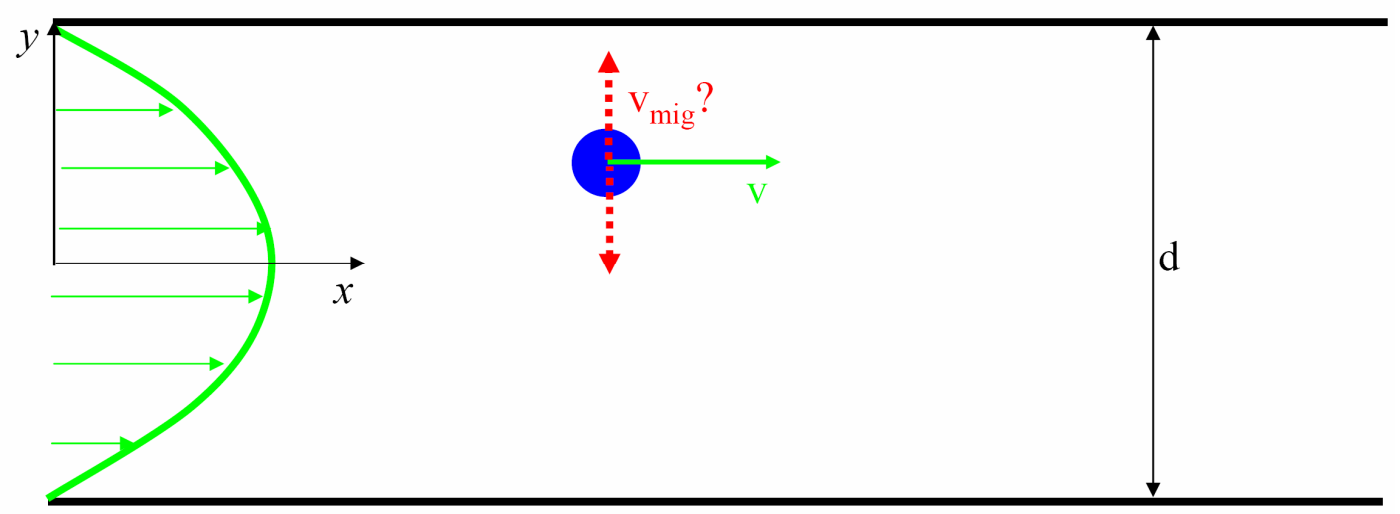

Figure 4-5: Migration of a particle inside a straight microchannel. The particle moves with velocity $v$ along the streamlines in the $x$-direction. The particle obtains a velocity $v_{m i g}$ in the $y$-direction for the case of cross-streamline migration.

In the 1830 s, Poiseuille observed an absence of red blood cells near walls during his studies of blood flow. This non-homogenous distribution of blood cells was related to migration perpendicular to flow direction. The observation was later confirmed by Fåhraeus and Lindquist (1931), who showed additionally that the migration of particles inside blood depends on their size [97]. Based on these studies, migration of nonbiological flow was investigated [98]. In 1962, Segré and Silberberg studied the radial distribution of rigid neutrally buoyant spheres flowing through circular tubes [99]. Surprisingly, they observed an accumulation of spheres at a radial position of approximately 0.6 pipe radii. Consequently, they found migration away from the centerline that was different from most of the former studies. Recently, accumulations of rigid spheres at a channel position between the channel wall and the centerline have 
been also observed inside microfluidic devices [100]. In microfluidics, cross-streamline migration can also be important for polymer solutions, such as for the existence of depletion layers near walls found in several experiments [23]. Studies with flexible polymers showed that the depletion layer near a single wall increases with larger flow velocities [25].

Considering a small particle in laminar flow inside a straight channel (see figure 4-5), the particle will primarily move along the streamlines. In this section, mechanisms are considered which create a drift and consequently migrate the particle away from the present streamline. If inertia is neglected, Bretherton showed that no force perpendicular to the flow direction can exist for a single rigid particle in unidirectional flow [101]. For vanishing Reynolds number, the Navier-Stokes equation results in the linear Stokes equation without an inertial contribution. If a rigid particle migrates in Poiseuille flow at zero Reynolds number toward the wall, the particle would migrate toward the centerline under flow reversal due to the reversibility resulting from the linearity of the Stokes equation [102]. However, the two scenarios are symmetric and therefore crossstreamline migration is forbidden at $R e=0$. In order to obtain a lateral force on the particle, a nonlinearity is necessary [103]. Nonlinearity can be generated by inertia (finite Reynolds number), which is considered in the next section. Deformation of particles can also induce nonlinearities and thereby create migration [103]. Furthermore, particle interactions for suspensions with finite volume fractions can become irreversible. In section 4.4.2, cross-streamline migration of a polymer illustrated by a dumbbell is considered for vanishing Reynolds numbers. In this case, the conformational changes of a polymer in flow as well as "particle interactions" of the different segments of a single polymer via hydrodynamic interactions are important.

\subsubsection{Inertial Migration}

In vertical tubes, heavier particles migrate in upwards flow toward the walls, whereas lighter particles migrate toward the centerline [104, 105]. For neutrally buoyant rigid spheres, experiments by Segré and Silberberg showed an accumulation at a radial position of approximately 0.6 pipe radii of the circular tubes (diameter: $\approx 1 \mathrm{~cm}$ ) [99]. These experiments were performed in the laminar flow regimes with Reynolds numbers $R e_{c}$ based on the channel width $d$ between 2 and 700. The results are confirmed by other experimental studies, for all of which the Reynolds number was of order unity or higher [104, 106, 107]. For example, Goldsmith and Mason showed that rigid particles are homogenously distributed across the channel for very low Reynolds numbers, and they migrate to a position between the wall and the centerline for finite Reynolds numbers 
[108]. While the former studies were all performed at the macroscopic scale with large tube dimensions (typically millimeter-centimeter in diameter), recent experiments showed that inertial migration can also be significant in microfluidics [100]. In microchannels (width $d \approx 50 \mu \mathrm{m}$ ), $9 \mu \mathrm{m}$ large spheres migrate to an equilibrium position in flow with velocities of about $\mathrm{m} / \mathrm{s}$. In this case, the particle Reynolds number is of order of one [100]. The particle Reynolds number in Poiseuille flow based on the size of the particle (diameter $a$ of the sphere) and the averaged shear rate is given by

$$
R e_{p}=\frac{\rho v_{o} a^{2}}{\eta d}
$$

where $v_{0}$ is the maximal velocity of the fluid at the centerline.

a)
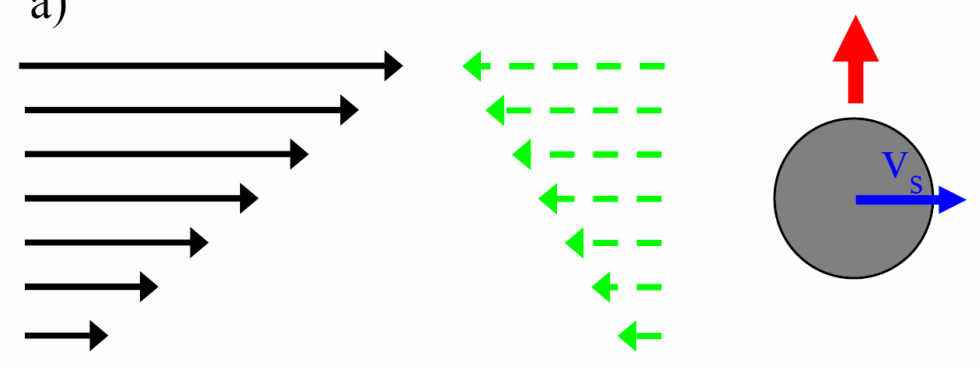

b)
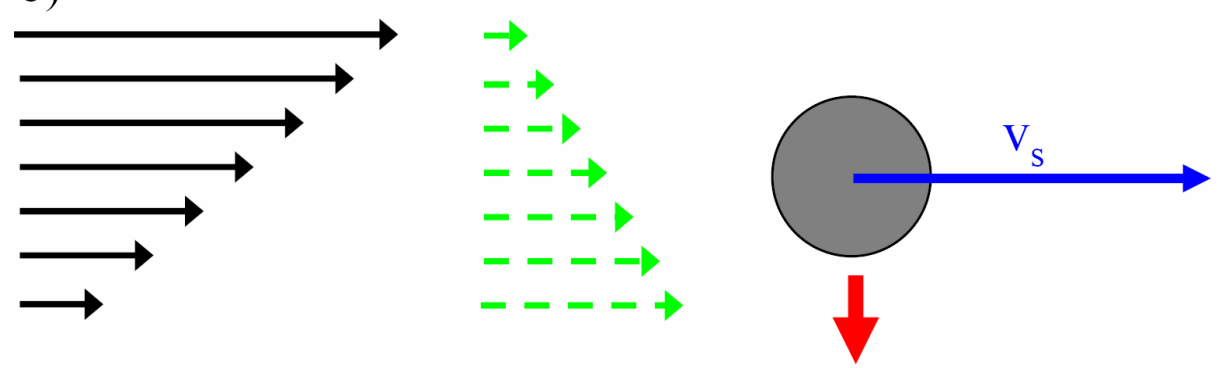

Figure 4-6: Migration of a sphere in simple shear flow at finite Reynolds numbers. The velocity of the sphere $v_{s}$ differs from the velocity of the fluid. The dashed green arrows illustrate the fluid flow relative to the sphere. a) A sphere moving slower than the fluid experiences a force in the direction of larger flow velocities. b) A sphere moving faster than the fluid experiences a force in the direction of lower flow velocities.

The experimental results for all studies mentioned above have been explained by inertial effects due to finite Reynolds numbers [109-116]. Brownian motion of the particles is not included in the considerations. In 1965, Saffman [115] illustrated how fluid inertia can induce a lateral force in simple laminar shear flow for a rigid sphere having a nonzero velocity relative to the fluid. He considered the scenario of a sphere in an unbounded fluid and calculated, via matched asymptotic expansion method, the lateral 
force. This force arises from the interaction of the disturbance flow created by the sphere and the velocity gradient. The magnitude of this force is given by [115]

$$
F_{s}=K a^{2} \dot{\gamma}^{1 / 2}(\eta \rho)^{1 / 2} v
$$

where $K \approx 6.46$ is a numerical constant and $v$ is the velocity of the sphere relative to the fluid. The direction of this lateral force is always toward regions where the fluid velocity relative to the particle is larger (see figure 4-6) [103, 110]. Due to the finite size of the particle, solvent molecules have to be displaced laterally when the sphere moves with a relative velocity to the fluid in flow direction. This displacement becomes irreversible at large distances away from the sphere because of inertia. The difference in the velocity of the displaced fluid from the background flow is larger for higher relative fluid velocities. Therefore, a pressure gradient along the sphere is created which generates a force in the direction of the larger relative velocity. This means that a particle moving faster than the fluid migrates toward slower flow (see figure 4-6b). Conversely, a particle moving slower experiences a force in the direction of the larger flow (see figure 4-6a).
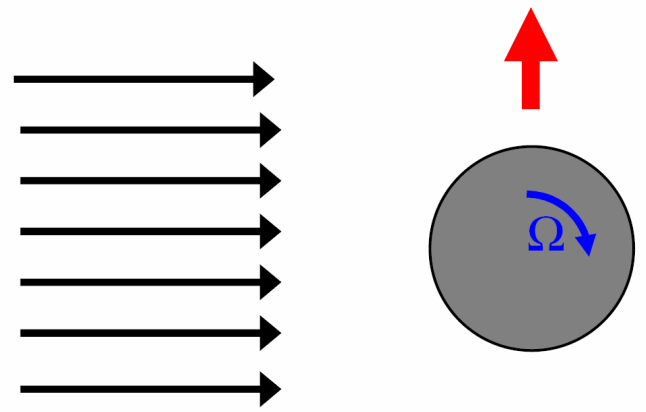

Figure 4-7: Cross-streamline migration due to rotation of a sphere in flow.

In simple shear flow, a neutrally buoyant sphere moves with the same velocity as the fluid and no Saffman force is induced. However, the Saffman force is relevant for nonneutrally buoyant spheres. A heavier sphere moving upwards in a vertical tube experiences a force toward the wall, while a lighter sphere experiences a force toward the centerline. This is qualitatively consistent with the experiments mentioned above [104, 105, 117].

Additionally, rotation of a sphere can also lead to migration analogous to the Magnus effect (figure 4-7). A pressure reduction is created by the rotational velocity at the side of the sphere where the velocity increases the fluid velocity. Consequently, the sphere will migrate in this direction. This lift force is given by [118]

$$
F_{R B}=\pi a^{3} \rho \Omega v,
$$

where $\Omega$ is the angular velocity and $v$ is the fluid velocity. In Poiseuille flow, the 
migration due to rotation of the sphere is always in direction toward the centerline [103]. Thus, this force cannot explain the migration of neutrally buoyant spheres away from the channel center, as was observed by Segré and Silberberg. An additional aspect has to be considered in Poiseuille flow, namely the curvature of the velocity field $[109,111$, 119]. Dividing the sphere with finite size in two halves, the side toward the wall has an overall larger relative velocity compared to the side toward the centerline (see figure 4-8). Consequently, the sphere migrates toward the channel wall by using the same argument considered for the Saffman force. If the sphere comes close to the wall, it is repelled from the wall due to inertial interactions with the wall $[110,119,120]$. The interplay between the wall repulsion and the migration away from the centerline due to the curvature of the velocity field creates an equilibrium position at a position between the wall and the centerline. For non-neutrally buoyant spheres, an additional Saffman contribution has to be considered.

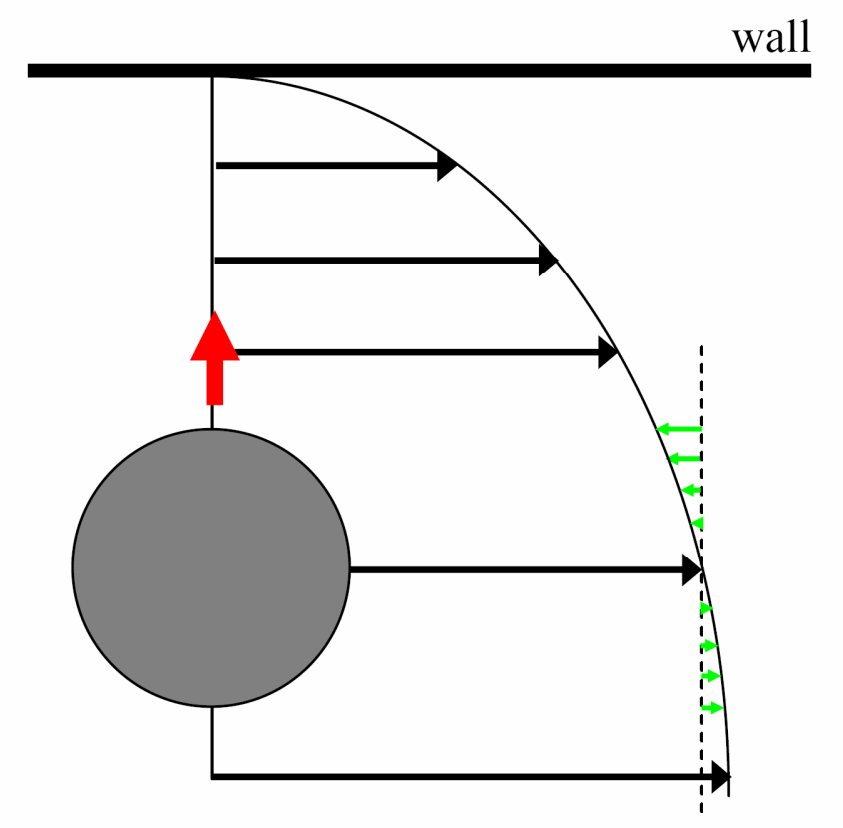

Figure 4-8: Inertial migration of a sphere in Poiseuille flow due to the curvature of the flow field. The sphere migrates in direction toward the channel wall. The green arrows illustrate the relative fluid velocity.

Most theoretical studies are based on solutions of the Navier-Stokes equation by using perpetuation methods [109-116]. At small distances around the sphere, the disturbance flow created by the sphere is to leading order determined by the Stokes equation. The viscous forces are dominant compared to inertial forces in this regime, the so-called inner region. However, they are on the same order at large distances away from the sphere (outer region). The Saffman force (equation 4.44) has been calculated for an 
unbounded fluid for which the inertial effects in the far field are responsible for its existence. However, the walls confine the fluid inside tubes. If the walls of the tube lie in the outer region, the particle motion is influenced by the inertial effects in the far field $[109,110]$. If the channel width is small enough that the walls are found in the inner region, irreversible inertial interactions with the walls are relevant. The length scales $l_{v}=\eta \rho / v_{0}=d / R e_{c}$ and $l_{s}=\left(\eta \rho d / v_{0}\right)^{1 / 2}=d / R e_{c}^{1 / 2}$ determine at which distance away from the particle the inertial effects from the far field become important for Poiseuille flow [109, 110, 116]. If the channel Reynolds number is of order of one, both length scales are comparable with the channel width and inertial effects from the far field are significant. Therefore, the theoretical studies can be classified by the channel Reynolds number [110] in regimes $R_{c}<<1$ [111, 112], $\operatorname{Re}_{c}=\mathrm{O}(1)[109,110,113,116]$, and $R e_{c}>>1[109,114,115]$. In all of these studies, the particle Reynolds number and the ratio of sphere diameter and channel width have been assumed to be small $\left(R e_{p}<<1\right.$ and $a / d<<1)$.

Although the experiments showing the migration of the sphere away from the centerline are performed with channel Reynolds numbers of unity or larger, the first theoretical theories in confined geometries considered the regime $R e_{c}<<1[111,112]$. Regular perpetuation techniques are used to obtain an expression for wall-induced inertial effects. The migration velocity can be calculated from [112]

$$
v_{\text {mig,v }}=\frac{\rho v_{0}^{2} a^{3}}{\eta d^{2}} f_{v}(y)=\frac{\eta}{\rho a} \operatorname{Re}_{p}^{2} f_{v}(y),
$$

where $f_{v}(y)$ is of order one and depends only on the channel position. $f_{v}(y)=0$ at exactly the middle between the wall and the centerline (channel position $y_{m}$ ). $f_{v}(y)$ is positive (which means migration toward the wall) between the centerline and $y_{m}$, and $f_{v}(y)$ is negative (migration toward the centerline) between the wall and $y_{m}$. Consequently, it can be expected that the particles migrate to the middle between the wall and the centerline.

For the regime $\operatorname{Re}_{c}=\mathrm{O}(1)$ in which the experiments have been performed, singular perpetuation methods are used in order to include inertial effects in the far field. The lateral force for a neutrally buoyant sphere is given by [109]

$$
F_{A}=\frac{\rho v_{0}^{2} a^{4}}{d^{2}} f_{A}\left(\operatorname{Re}_{c}, y\right)=\frac{\eta^{2}}{\rho} \operatorname{Re}_{p}^{2} f_{A}\left(\operatorname{Re}_{c}, y\right),
$$

where $f_{A}\left(R e_{c}, y\right)$ is a coefficient which dependent on channel position and channel Reynolds number but not on the particle size. $f_{A}\left(R e_{c}, y\right)$ is positive at the channel center (migration toward the wall) and negative at the channel wall (migration toward the centerline). $f_{A}\left(R e_{c}, y\right)$ decreases monotonically from the channel center toward the 
wall and is zero at the equilibrium position. For rotational spheres, a correction can be made to include the migration toward the centerline due to the rotation of the sphere [109]. This correction is only small because the force due to rotation is one order of magnitude smaller than the force in equation 4.47 [110]. Balancing the lateral force with the Stokes drag force $F_{d r a g}=3 \pi \eta a v$, the migration velocity is given by [100]

$$
v_{m i g, A}=\frac{\rho v_{0}^{2} a^{3}}{3 \pi \eta d^{2}} f_{A}\left(\operatorname{Re}_{c}, y\right)=\frac{\eta}{3 \pi \rho a} \operatorname{Re}_{p}^{2} f_{A}\left(\operatorname{Re}_{c}, y\right) .
$$

This expression converges asymptotically for decreasing channel Reynolds numbers toward equation 4.46 [29]. The migration velocity depends on particle size, and decreases strongly for smaller particles. This might explain why migration was only observed in the above mentioned microfluidic experiments when the particle Reynolds number is of order of one [100].

The expressions 4.46 and 4.48 have been calculated for rigid spheres of diameter $a$. In chapter 5 , we consider the cross-streamline migration of semiflexible actin filaments (diameter $\approx 7 \mathrm{~nm}$, length $\approx 8 \mu \mathrm{m})$ in aqueous solution $(\rho \approx 1 \mathrm{~kg} / \mathrm{L}, \eta \approx 1 \mathrm{mPa} \cdot \mathrm{s})$ inside microchannels (width $d \approx 10 \mu \mathrm{m}$, length $\approx 2 \mathrm{~cm}$ ) at flow velocities of about $\mathrm{mm} / \mathrm{s}$. The Reynolds numbers based on the channel width are $R e_{c} \approx 10^{-3} \ldots 10^{-2}$. Considering an actin monomer (length $\approx$ diameter $\approx 7 \mathrm{~nm}$ ) as a rigid sphere, the migration velocity $v_{\text {mig }} \approx 3 \cdot 10^{-15} \mathrm{~m} / \mathrm{s}$ can be estimated by equation 4.46 . The diameter of a sphere having the same volume $V \approx 3 \cdot 10^{-22} \mathrm{~m}^{3}$ (mass: $\approx 3 \cdot 10^{-19} \mathrm{~kg}$ ) as a filament is $d=80 \mathrm{~nm}$. Such a sphere migrates with velocity $v_{\text {mig }} \approx 5 \cdot 10^{-12} \mathrm{~m} / \mathrm{s}$. Therefore, inertial effects can be neglected for cross-streamline migration. However, a description of polymer migration has to include conformational changes due to the flow field, Brownian motion, and "particle-particle" interactions (i.e. interactions between the segments of a single polymer).

\subsubsection{Migration of Polymers}

Depletion layers of polymers near walls were observed in several experiments and were explained by cross-streamline migration of polymers away from the wall [23]. Additionally, computer simulations of flexible polymers as well as of stiff Brownian rods suggest migration away from the centerline in Poiseuille flow [26, 29-31, 121-124]. In the previous section, the cross-streamline migration of non-Brownian rigid particles at finite Reynolds numbers has been considered for which inertial effects are important. Other mechanisms are discussed for polymers in flow at low Reynolds numbers [23, 26, 27, 29-33, 121-126]. In this section, the migration of polymers at vanishing Reynolds numbers is illustrated by a dumbbell model. 


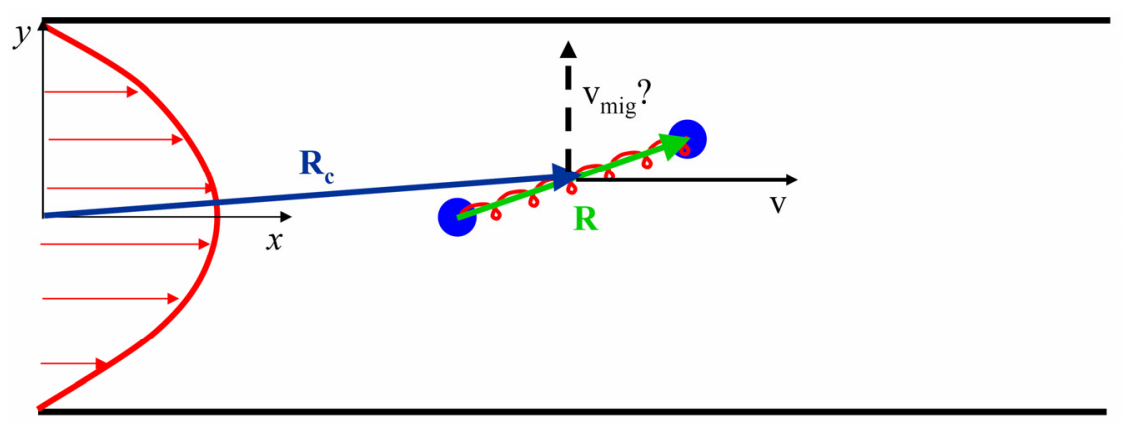

Figure 4-9: Migration of a dumbbell inside a straight microchannel. The dumbbell (R: end-to-end vector, $\mathbf{R}_{\mathrm{c}}$ : center-of-mass vector) moves with velocity $v$ along the streamlines in the $x$-direction and obtains a velocity $v_{m i g}$ perpendicular to flow direction in the case of cross-streamline migration.

Considering a Brownian dumbbell inside a channel, one motion, moving the dumbbell away from the present streamline, is diffusion. Normal Fickian diffusion has no preferred direction and a dumbbell diffuses with the same probability up or down $(y$ direction in figure 4-9). Neglecting steric exclusion effects near walls, the probability to find a dumbbell with random initial conditions at a position $y$ inside the channel is for all positions the same and is independent from the flow strength. This section explains mechanisms that create a drift and consequently migrate the dumbbell away from the present streamline. This leads to an inhomogeneous center-of-mass probability distribution for a dumbbell inside the microchannel.

In section 4.3.2, the dumbbell model is introduced and forces (drag force, Brownian force, hydrodynamic interactions, and spring force) acting on the beads are considered. To study cross-streamline migration, hydrodynamic interactions are important [29], which are neglected in the consideration of the orientation of a dumbbell in shear flow (section 4.3.3). Furthermore, the diffusion coefficient is allowed to depend on the conformation of the dumbbell [127]. As before, the dumbbell is described by its end-toend vector $\mathbf{R}$ and its center-of-mass vector $\mathbf{R}_{c}$. For a dumbbell with random initial conditions, the probability of each possible state characterized by $\mathbf{R}$ and $\mathbf{R}_{c}$ at a time $t$ is given by the configurational probability distribution function $\Psi\left(\mathbf{R}_{c}, \mathbf{R}, t\right)$, which is determined by the governing equation [29]

$$
\frac{\partial \Psi}{\partial t}=-\frac{\partial}{\partial \mathbf{R}_{c}}\left(\dot{\mathbf{R}}_{c} \Psi\right)-\frac{\partial}{\partial \mathbf{R}}(\dot{\mathbf{R}} \Psi)
$$

The velocity of the center-of-mass $\dot{\mathbf{R}}_{c}$ is determined by equation 4.23 and the rate of change of the end-to-end vector $\dot{\mathbf{R}}$ is determined by equation 4.22 , where the Brownian force at the $i$-th bead is given by [26]:

$$
\mathbf{F}_{\text {brown }, i}=-k_{b} T \frac{\partial}{\partial \mathbf{R}_{i}} \ln \Psi .
$$


The center-of-mass probability distribution $n\left(\mathbf{R}_{c}, t\right)$, which describes the position of the dumbbell inside the microchannel, is obtained by integrating the configurational probability distribution function $\Psi\left(\mathbf{R}_{c}, \mathbf{R}, t\right)$ over all possible end-to-end vectors [29]

$$
n\left(\mathbf{R}_{c}, t\right)=\int \Psi\left(\mathbf{R}_{c}, \mathbf{R}, t\right) \mathrm{d} \mathbf{R} .
$$

Defining

$$
\Psi\left(\mathbf{R}_{c}, \mathbf{R}, t\right)=n\left(\mathbf{R}_{c}, t\right) \hat{\Psi}\left(\mathbf{R}_{c}, \mathbf{R}, t\right),
$$

integration of equation 4.49 over $\mathbf{R}$ gives the governing equation for the center-of-mass probability distribution [26]

$$
\frac{\partial n\left(\mathbf{R}_{c}, t\right)}{\partial t}=-\frac{\partial}{\partial \mathbf{R}_{c}} \mathbf{j}_{c},
$$

where $\mathbf{j}_{c}=\left\langle\dot{\mathbf{R}}_{c}\right\rangle n$ is the center-of-mass flux, and the angle bracket designates an ensemble average over the configuration variable $\mathbf{R}$ and is defined, more generally, for any variable $A$ as

$$
\langle A\rangle=\int A \hat{\Psi} \mathrm{d} \mathbf{R} .
$$

Multiplying the governing equation of the center-of-mass vector (equation 4.23) with $\Psi$, and integrating over $\mathbf{R}$, the center-of-mass flux can be calculated yielding [29]

$$
\begin{aligned}
\mathbf{j}_{c} & =\left[\left\langle\mathbf{v}_{c}\right\rangle+\frac{1}{2}\left\langle\overline{\mathbf{\Omega}} \cdot \mathbf{F}_{\text {spring }}\right\rangle\right] n\left(\mathbf{R}_{c}, t\right) \\
& -\frac{1}{4}\left[2\left\langle\frac{\partial}{\partial \mathbf{R}} \cdot\left(\mathbf{D}_{11}-\mathbf{D}_{22}\right)\right\rangle-\left\langle\frac{\partial}{\partial \mathbf{R}_{c}} \cdot\left(\mathbf{D}_{11}+\mathbf{D}_{22}\right)\right\rangle+\frac{\partial}{\partial \mathbf{R}_{c}} \cdot\left\langle\mathbf{D}_{k}\right\rangle\right] n\left(\mathbf{R}_{c}, t\right) \\
& -\frac{1}{4}\left\langle\mathbf{D}_{k}\right\rangle \cdot \frac{\partial n\left(\mathbf{R}_{c}, t\right)}{\partial \mathbf{R}_{c}},
\end{aligned}
$$

where $\mathbf{D}_{K}=\left(\mathbf{D}_{11}+\mathbf{D}_{22}\right)+\left(\mathbf{D}_{21}+\mathbf{D}_{12}^{T}\right)$ and $\overline{\boldsymbol{\Omega}}=\left(\boldsymbol{\Omega}_{11}-\boldsymbol{\Omega}_{22}\right)+\left(\boldsymbol{\Omega}_{21}-\boldsymbol{\Omega}_{12}\right) .\left\langle\mathbf{D}_{K}\right\rangle$ is the so-called Kirkwood diffusivity for a dumbbell. The Kirkwood diffusivity is often used as the diffusion coefficient for a polymer [89].

The steady state solution of the center-of-mass probability distribution is determined by (see equation 4.53)

$$
-\frac{\partial}{\partial \mathbf{R}_{c}} \mathbf{j}_{c}=0
$$

where the flux $\mathbf{j}_{c}$ is given by equation 4.55 . In principle, the center-of-mass probability distribution in steady-state is obtained by solving this equation.

The last term in equation 4.55 is the normal Fickian diffusion, which is proportional to the gradient of the center-of-mass probability distribution (gradient of the "concentration"). All other terms, which are proportional to the center-of-mass 
probability distribution, can lead to migration. The first line in this equation represents a deterministic contribution to migration and the second a Brownian contribution to migration.

The first term in the first line of equation 4.55 is the contribution from the imposed flow field. In rectilinear channels, the components of the flow field in wall-normal direction are zero and therefore no cross-streamline migration can be induced directly by the imposed flow field.

In the presence of a wall, the second term in the first line of equation 4.55 leads to migration of the dumbbell away from the wall due to hydrodynamic interactions of the dumbbell with the wall $[26,29]$. A stretched dumbbell induces a flow field that is not symmetric because of the broken symmetry in the geometry near a wall. This situation is illustrated in figure 4-10. Due to shear, the dumbbell becomes stretched and orientated parallel to the streamlines of the fluid. The dumbbell wants to relax back, and thereby creates a point force at each bead which acts on the solvent molecules of the fluid. The generated flow field is symmetric in the case of no walls (see figure 4-10a) and the dumbbell has no tendency to move up or down. In confining geometries (see figure $4-10 \mathrm{~b}$ ), the flow field generated by hydrodynamic interactions is no longer symmetric. Near walls, the no-slip boundary condition "deforms" the flow field. In a simple picture, the flow is reflected at the walls. Then, the dumbbell obtains a deterministic drift away from the wall.

In inhomogeneous flow, the second line in equation 4.55 can also lead to crossstreamline migration $[29,32,33]$. The first two terms are contribution due to variations in the bead mobility at different channel positions. When the influence of the wall on the bead mobility is neglected, the bead mobility is constant and these two terms vanish [29]. The third term is due to spatial variations in the dumbbell diffusivity. In general, the diffusivity of a dumbbell depends on its conformation [29], and the conformation in spatially non-uniform flow fields can be strongly position-dependent. In pressure-driven flows, the different local shear rates lead to position-dependent averaged stretching of a dumbbell. Thus, the diffusivity of a dumbbell varies along the cross-section of the channel, resulting in migration of the dumbbell away from the centerline [29]. This situation is illustrated in figure 4-10b. The dumbbell is more stretched away from the centerline than at the centerline, where the local shear rate is zero. The diffusion constant $\mathbf{D}_{1}$ of a not-stretched dumbbell at the centerline is larger than the diffusion constant $\mathbf{D}_{2}$ of a stretched dumbbell closer to the wall. A dumbbell diffuses faster away from the centerline than toward the centerline which leads to migration toward the wall. 
a)

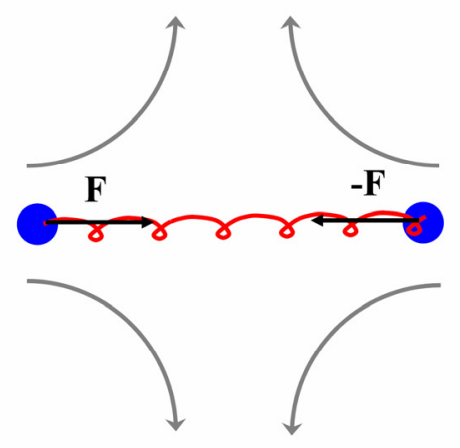

b)

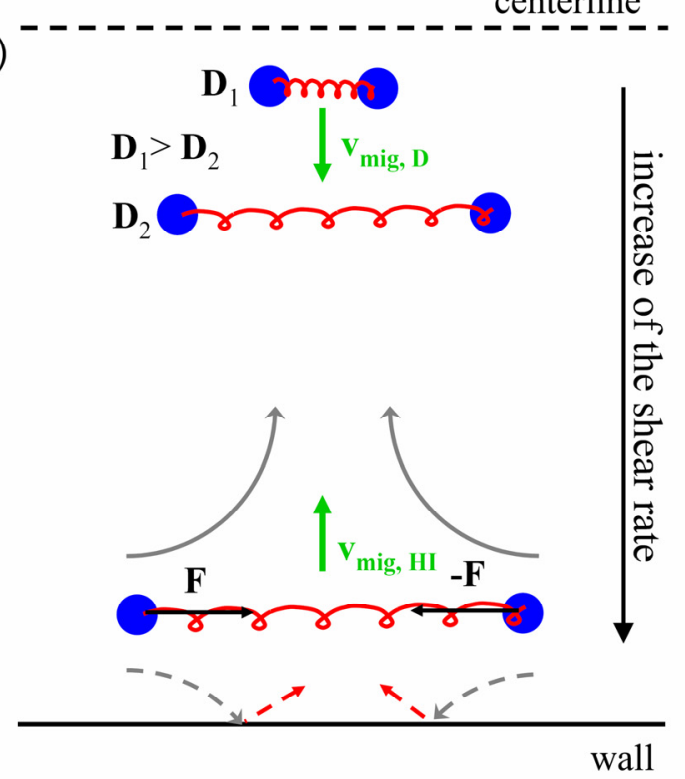

Figure 4-10: a) Hydrodynamic interactions of a dumbbell in bulk fluid. Relaxation of the dumbbell induces a symmetric microflow. b) Migration of a dumbbell in pressure-driven flow in confining geometry. Relaxation of the dumbbell induces an asymmetric microflow near the wall, which moves the polymer away from the wall. Different local shear rates inside the channel leads to positiondependent averaged stretching of the dumbbell. This leads to a spatially-varying diffusivity and to migration away from the centerline.

To summarize, two different migration mechanisms influence the center-of-mass probability distribution in rectilinear channels for dumbbells in pressure-driven flow: hydrodynamic interactions with walls lead to migration away from the walls and spatially-varying diffusivity leads to migration away from the centerline. Neglecting spatial-variations in bead mobility, the governing equation for the center-of-mass probability distribution (equations 4.53 and 4.55 ) can be simplified in the twodimensional case for a straight channel. The center-of-mass probability distribution perpendicular to flow direction ( $y$-direction in figure 4-9) is determined for any time $t$ by

$$
\frac{\partial n}{\partial t}=-\frac{\partial}{\partial y_{c}} j_{c, y}
$$

where

$$
j_{c, y}=\frac{1}{2}\left\langle\overline{\mathbf{\Omega}} \cdot \mathbf{F}_{\text {spring }}\right\rangle n\left(y_{c}, t\right)-\frac{1}{4} \frac{\partial}{\partial y_{c}}\left\langle\mathbf{D}_{k}\right\rangle n\left(y_{c}, t\right)-\frac{1}{4}\left\langle\mathbf{D}_{k}\right\rangle \cdot \frac{\partial n\left(y_{c}, t\right)}{\partial y_{c}} .
$$

At steady-state, migration is balanced by diffusion. 


\section{Chapter 5}

\section{Semiflexible Filaments in Pressure- Driven Flow}

In this chapter, measurements of actin filaments in flow inside microfluidic channels are discussed. Experiments are performed by using fluorescence microscopy, allowing the direct observation of the contours in flow. Dilute solutions ensure that interactions between different filaments are negligible and the approach can be focused on single polymer dynamics. Actin filaments are semiflexible polymers with a persistence length of $L_{p} \approx 13 \mu \mathrm{m}$ [5] and typical contour length in the experiments of $L \approx 8 \mu \mathrm{m}$. The width and depth of the microchannels are about $10 \mu \mathrm{m}$. Hence, all important length scales of the observed system have approximately the same magnitude. The orientations and conformations of actin filaments at different channel positions and velocities are analyzed. Furthermore, the frequencies of filaments at certain channel positions along a cross-section of the channel are measured and information about cross-streamline migration is obtained.

\subsection{Experiment}

The used microchannels have a depth of $h=10 \pm 1 \mu \mathrm{m}$ and width of $d=11 \pm 1 \mu \mathrm{m}$, and therefore the aspect ratio (depth/width) is close to unity (see figure 5-1). After filling the channels with a dilute solution of actin filaments $(70 \mathrm{nM})$, a pressure-driven flow is created (see section 2.3). The channels have a length of about $2 \mathrm{~cm}$, and the actin filaments are analyzed at the end of the channels. Using FemLab, a commercially available software (FemLab GmbH, Göttingen), the flow field is simulated and is shown in figure 5-2. Although the flow field can be calculated analytically [128], it is 
approximated for the following discussions by the Poiseuille flow (see figure 5-2b) in order to obtain a simple expression. For Poiseuille flow, the solvent velocity at $z=0$ is given by

$$
\mathbf{v}=v_{0}\left(1-\frac{y^{2}}{(d / 2)^{2}}\right) \mathbf{e}_{x}
$$

where $\mathbf{e}_{x}$ is the unit vector in $x$-direction, $v_{0}$ is the maximal velocity inside the channel and $d$ is the channel width. In this approximation, the local shear rate (see equation 4.16)

$$
\dot{\gamma}(y)=\frac{2 v_{0}}{(d / 2)^{2}}|y|
$$

is proportional to the channel position $y$ with a minimum $\dot{\gamma}=0$ at the centerline.

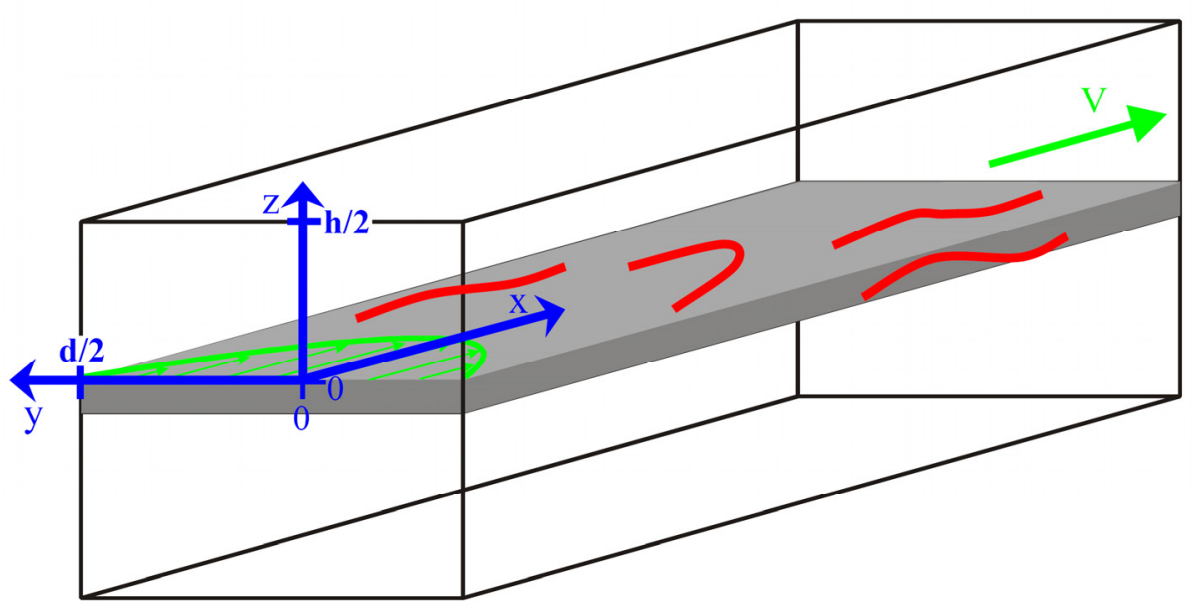

Figure 5-1: Flowing actin filaments inside microchannels with width $d$ and depth $h$. The $x$-axis points in flow direction and the $y$-and $z$-axis are perpendicular to the flow direction. The origin of the coordinate system is at the channel center $z=0$ and $y=0$. The filaments are observed in a plane $\pm 0.5 \mu \mathrm{m}$ at $\mathrm{z}=\mathbf{0}$ (grey area).
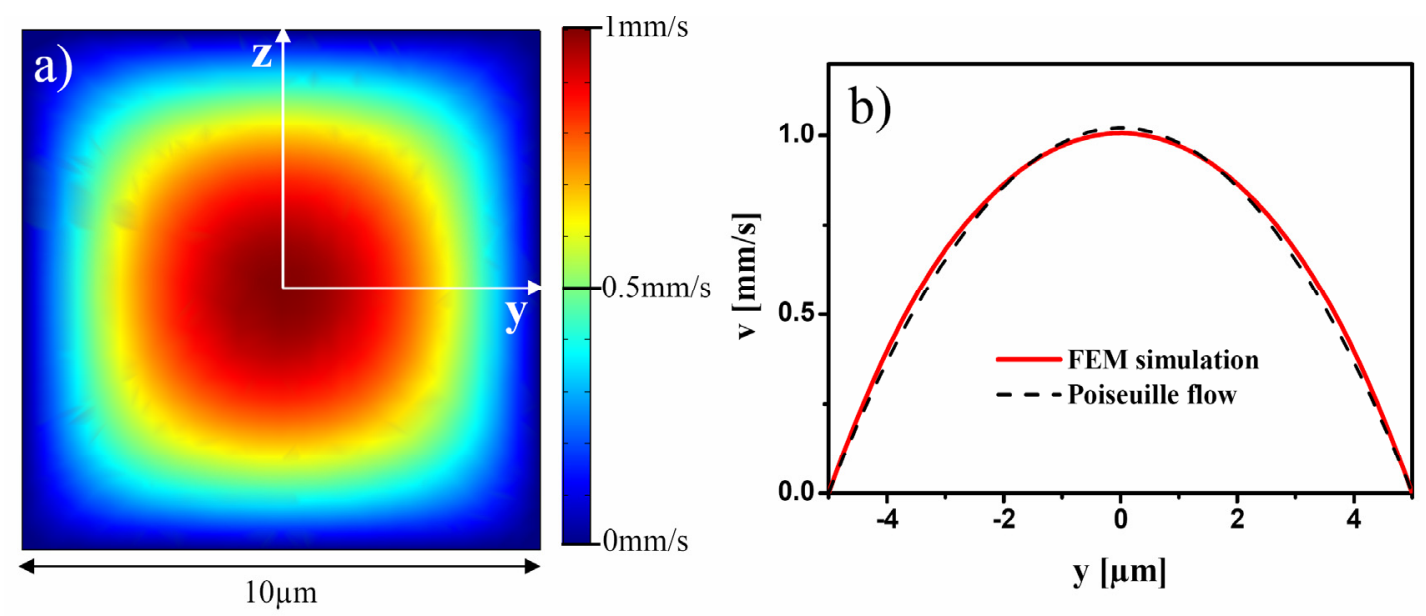

Figure 5-2: FEM-simulation (finite element method) of the flow field. a) Slice of the flow field at $x=$ const. b) Velocity profile in $y$-direction at $z=0$ and $x=$ const. 
The contour lengths of the actin filaments inside the dilute solution varies, and the solution always contains some very short actin filaments $(<1 \mu \mathrm{m})$. These short filaments, whose velocities differ only little from the velocity of the solvent, are used to determine the velocity of the fluid in the experiments. The velocities of many short filaments at the channel center are measured and the averaged value is taken for the maximal velocity $v_{0}$. The microscope is focused to the half the channel height (grey area in figure 5-1). In the analysis, we consider only filaments which have a relatively sharp contour and thus coincide more or less with their 2D projection (i.e. which are mainly located in the focal plane $\pm 0.5 \mu \mathrm{m})$. Typical shapes of flowing actin filaments inside a microchannel are shown in figure 5-3. With the exception of filaments in the channel center, filaments are mostly elongated and aligned in the flow direction (see figure 5-3a). Furthermore, tumbling of filaments is observed (see figure 5-3b). In section 5.4, the behavior of these filaments is investigated in more detail. At the channel center, a parabolic shape of actin filaments can often be seen (figure 5-3c). This shape will be analyzed in section 5.4.3.
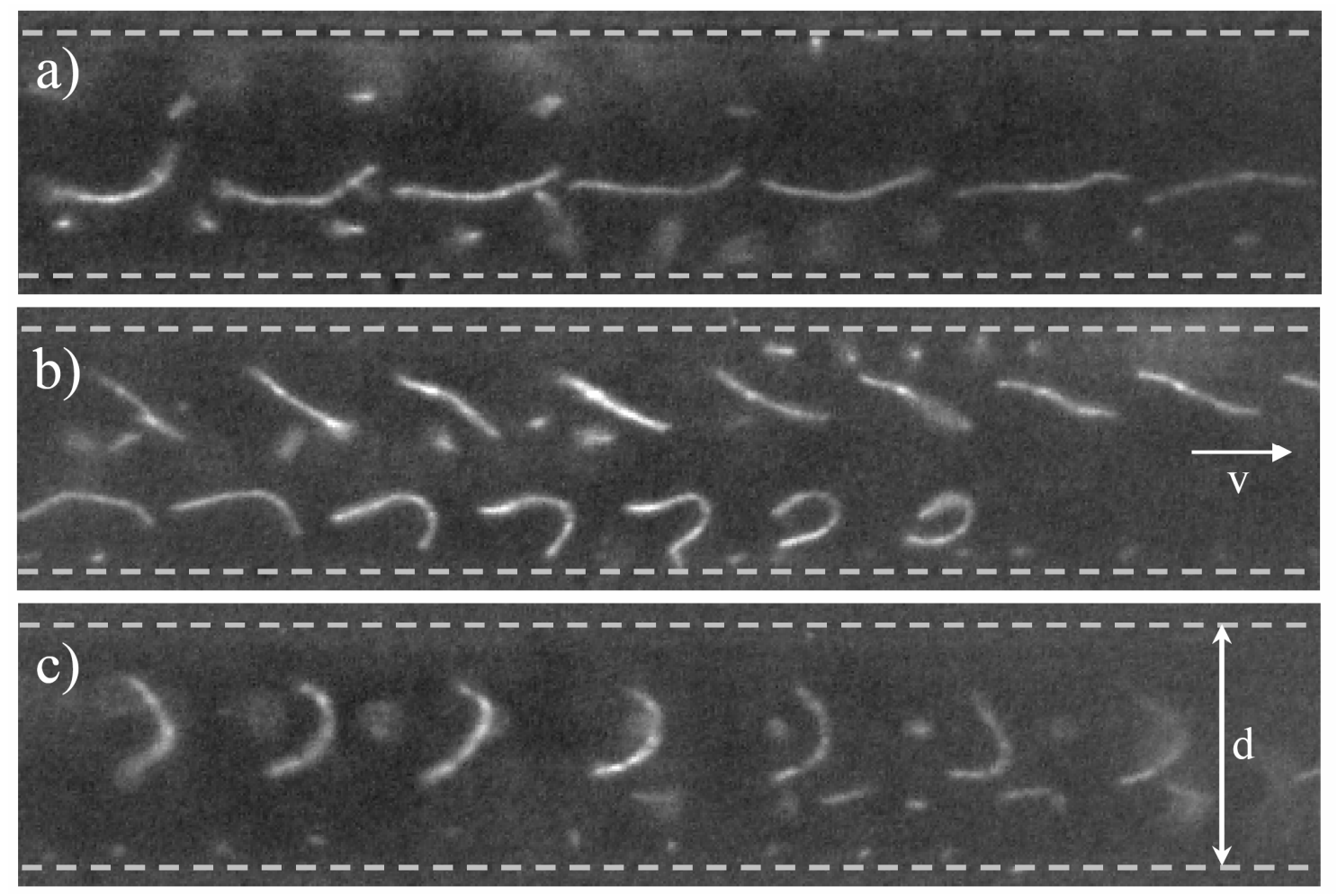

Figure 5-3: Typical shapes of actin filaments in flow (velocity: $v_{0} \approx 500 \mu \mathrm{m} / \mathrm{s}$ ) in microchannels (width $d=11 \mu \mathrm{m}$ ): a) elongated filament, b) tumbling filament and c) parabolically bent filament. The images are obtained using stroboscopic illumination (time period: $\Delta t \approx 30 \mathrm{~ms}$ ).

The influence of confining geometry on orientation and conformation is small for actin filaments in microchannels of about $10 \mu \mathrm{m}[5,6]$. Actin filaments (contour length of 
about $8 \mu \mathrm{m}$ ) can rotate freely inside the channels. This allows us to treat actin filaments as free polymers and alignment and conformation changes can be directly interpreted in the context of flow.

Without flow, the semiflexible filaments are randomly orientated and the thermal fluctuations are large. The entire contours of the filaments are often not completely found in the focal plane. Thus, the imaging of actin filaments without flow can be problematic and imprecise. However, fluctuations of the shape are suppressed in flow and the actin filaments are predominantly aligned and elongated (see figure 5-3 and for more details section 5.4). The extension perpendicular to the observed plane (z-direction in figure 5-1) decreases with larger velocity of the flowing solution, and the 2Ddescription of the shape converges toward the actual 3D-shape of the filaments. Thus, the error in the following analysis decreases with larger velocities.

\subsection{Data Analysis}
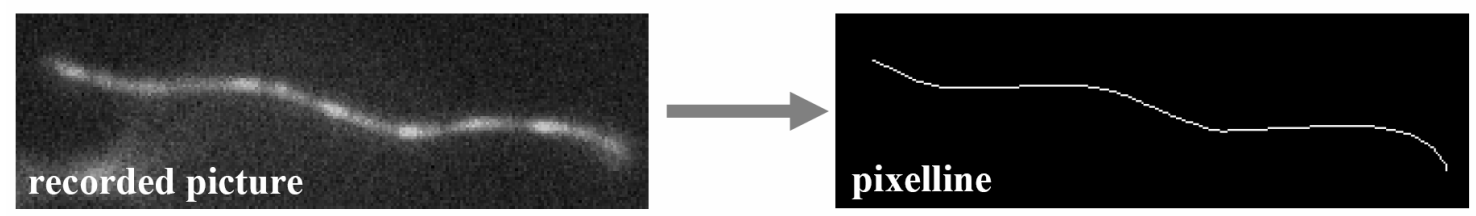

Figure 5-4: Creating a pixelline from the recorded picture.

Starting with the recorded picture, a pixelline of the contour is drawn (see figure 5-4), using several image filters. These are basically a median filter to reduce noise, a binarization filter to create a black-white image, and a skeletonization filter to create a line with a width of one-pixel (for details see [5]). The image processing is performed using a combination of software packages (AnalySIS (Soft Imaging Systems, Münster), ImageProPlus (Media Cybernetics, Silver Spring, USA) and Adobe Photoshop (Adobe Systems, San Jose, USA)). Analyzed parameters of the pixelline (see figure 5-5 for definitions) are measured using a MatLab (The Mathworks, Natick, USA) macro.

The first parameter considered for each filament is the contour length $L$. Due to polydispersity, the length of the recorded actin filaments varies. In order to keep polymer properties constant, the analysis includes only filaments with approximately the same contour length (averaged contour length: $8 \pm 2 \mu \mathrm{m}$ ) and neglects all shorter or longer ones. For each velocity, around 1000 filaments are analyzed. 


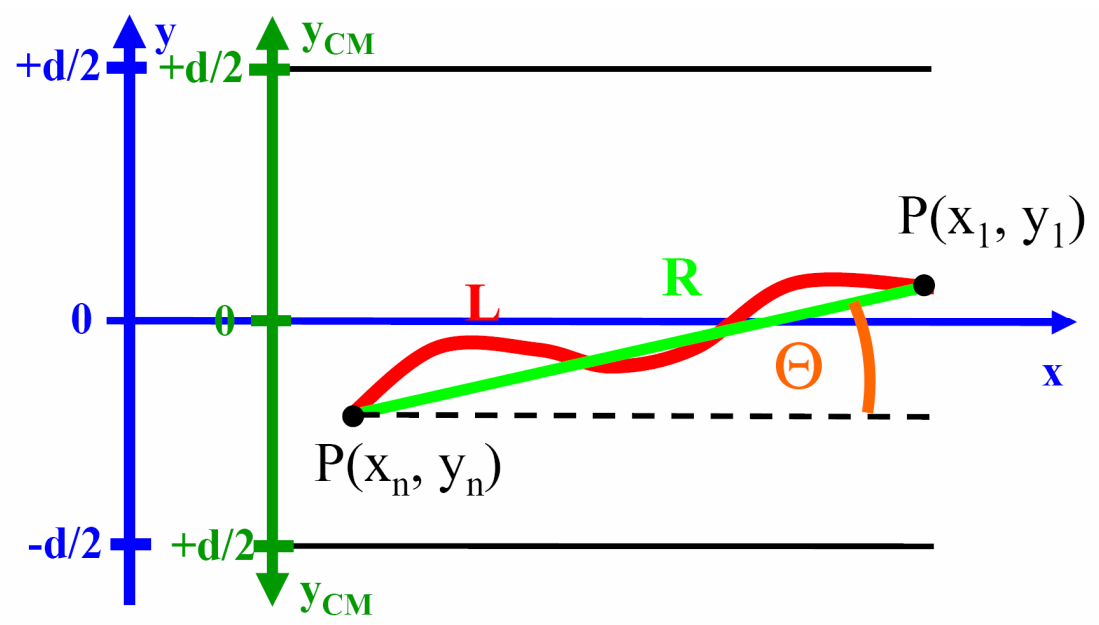

Figure 5-5: Definition of the parameters. $x$-axis: in flow direction. $y$-axis: perpendicular to the flow direction with origin at the channel center and walls at $y= \pm d / 2 . L$ : contour length of the actin filament. $R$ : end-to-end distance. $\mathrm{P}\left(x_{1}, y_{1}\right)$ and $\mathrm{P}\left(x_{n}, y_{n}\right)$ : endpoints of the contour $\left(\mathrm{P}\left(x_{n}, y_{n}\right)\right.$ is the end with the smaller $x$-value $\left.\left(x_{n}<x_{1}\right)\right)$. $\Theta$. angle of the end-to-end vector relative to the flow direction.

To measure the elongation of filaments, the end-to-end distances $R$ between both ends at positions $\mathrm{P}\left(x_{1}, y_{1}\right)$ and $\mathrm{P}\left(x_{n}, y_{n}\right)$ are determined:

$$
R=\sqrt{\left(x_{1}-x_{n}\right)^{2}+\left(y_{1}-y_{n}\right)^{2}} .
$$

The positions of filaments inside the microchannels are measured by the center-of-mass in the $y$-direction

$$
y_{c m}=\left|\frac{1}{n} \sum_{i=1}^{n} y_{i}\right|,
$$

where $y_{i}$ is the position of the $i$-th pixel and $n$ is the total number of pixels in the pixelline. All filaments are measured and analyzed across the entire channel in the $y$ direction. To improve the statistics, we project the data onto one channel half. This is achieved by taking the absolute value for the center-of-mass in equation 5.4.

The angle $\Theta$ of the end-to-end vector relative to the flow direction describes the orientations of actin filaments within the channel. We define that the angle $\Theta$ is positive, if the endpoint $\mathrm{P}\left(x_{\mathrm{n}}, y_{\mathrm{n}}\right)$ with the smaller $x$ value is closer to the walls than the other endpoint $\mathrm{P}\left(x_{1}, y_{1}\right)$ :

$$
\begin{aligned}
& \left|y_{n}\right|>\left|y_{1}\right| \rightarrow \Theta>0 \\
& \left|y_{1}\right|<\left|y_{n}\right| \rightarrow \Theta<0 .
\end{aligned}
$$

To determine the averaged orientation of the filaments, an orientational order parameter

$$
S=\left\langle\frac{3 \cos ^{2} \Theta-1}{2}\right\rangle
$$

is calculated [89]. 
If this order parameter is zero $(S=0)$, the filaments are randomly orientated over all possible angles $\left(-90^{\circ} \leq \Theta \leq 90^{\circ}\right)$. In this case $\left\langle\cos ^{2} \Theta\right\rangle=1 / 3$, resulting in an averaged angle of $\Theta \approx 55^{\circ}$ for free polymers in the 3-dimensional case. The more the filaments are aligned, the more the order parameter increases, and in the situation where all filaments are perfectly parallel, the order parameter is one $(S=1)$.

\subsection{Relaxation of Actin Filaments}

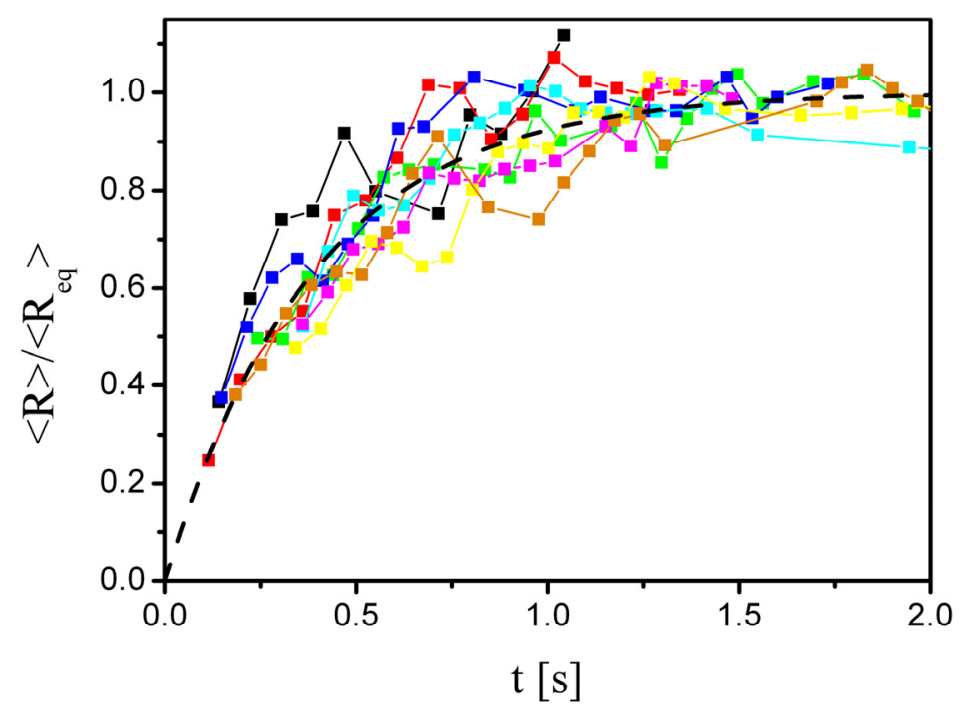

Figure 5-6: Relaxation of bent actin filaments. Different colors represent individual filaments. The plotted line represents the function $f(t)=1-\exp (-t / 0.4 \mathrm{~s})$.

Actin filaments in flow are elongated or bent (see figure 5-3). Stopping the flow, filaments relax back to equilibrium on a characteristic time scale. This relaxation time is an important property for the description of polymer deformations in flow. Comparison of relaxation time and flow deformation time, which can be expressed by the Weissenberg number (see chapter 4.3.1), specifies how strongly the polymer is deformed in flow.

To measure the relaxation time of actin filaments, a flowing solution $(v>1 \mathrm{~mm} / \mathrm{s})$ is stopped abruptly (within $\sim 50 \mathrm{~ms}$ ) and snapshots of the shapes are taken in time intervals of $\Delta t=80 \mathrm{~ms}$. The end-to-end distances $R$ of initially bent filaments are measured and the evolution for different filaments is shown in figure 5-6. The curves are normalized by an averaged end-to-end distance in equilibrium, which is measured at long times. The initial, small normalized end-to-end distances of bent filaments increase with time until they fluctuate around a value of one. 
All relaxation curves are fitted with an exponential function

$$
f(t)=1-\exp \left(-\frac{t-t_{0}}{\tau_{r e l}}\right),
$$

where $t_{0}$ and the relaxation time $\tau_{\text {rel }}$ are fit parameters. The time $t_{0}$ varies for different analyzed filaments because they have slightly different starting conditions depending on how strongly the filaments are bent at the beginning of the observation. In figure 5-6, the curves of the individual filaments are shifted by the value of $t_{0}$ for better comparison, so that all curves converge.

Averaging the relaxation times for all analyzed filaments results in $\tau_{\text {rel,exp }}=(0.4 \pm 0.2) \mathrm{s}$. The large error arises from imprecisely measured positions of the endpoints, which can often be only roughly estimated in the recorded pictures. Actin filaments, which at the beginning of the measurement are found almost exclusively in the focal plane, often move away from the focus during relaxation due to their randomly oriented and largely fluctuating 3-dimensional conformations in equilibrium.

The relaxation process can be described by balancing drag force and bending force at each segment of the filament at every time. A relaxation time [81]

$$
\tau_{r e l}=\frac{c_{f}}{L_{p} k_{B} T}\left(\frac{L}{\pi\left(n_{0}+1 / 2\right)}\right)^{4}
$$

can be derived, where $c_{f}$ is the drag coefficient per length and $n_{0}$ marks different hydrodynamic modes. With persistence length $L_{p}=13 \mu \mathrm{m}$ [5], contour length $L=8 \mu \mathrm{m}$, Boltzmann constant $k_{B}=1.4 \cdot 10^{-23} \mathrm{~J} / \mathrm{K}$ [87], temperature $T=293 \mathrm{~K}$ and drag coefficient per length $c_{f}=1.8 \mathrm{mPas}$, the longest relaxation time $\left(n_{0}=1\right)$ can be calculated to be $\tau_{\text {rel, theo }} \approx 0.3 \mathrm{~s}$. The drag coefficient $c_{f}=1.8 \mathrm{mPas}$ of actin in water (viscosity $\eta=1 \mathrm{mPas}$ ) is calculated (equation 4.4) by considering actin filaments in a first approximation as a rigid cylinder with length $L=8 \mu \mathrm{m}$ and diameter $d=7 \mathrm{~nm}$. The calculated relaxation time $\tau_{\text {rel, theo }} \approx 0.3 \mathrm{~s}$ agrees with the experimental determined relaxation time $\tau_{\text {rel,exp }}=0.4 \pm 0.2 \mathrm{~s}$ within its error range.

The strong dependence of relaxation time on contour length in equation 5.8 (i.e. with the power of four) points out a problem for determining the relaxation time in our experimental system. Although in the analysis we include only filaments having approximately the same length $(L=8 \pm 2 \mu \mathrm{m})$, the relaxation time of each individual actin filament is very sensitive to slight differences in length. Nevertheless, a mean relaxation time of $\tau_{\text {rel }} \approx 0.3-0.4 \mathrm{~s}$ is reasonable.

As mentioned above, the relaxation time is mainly needed to determine the Weissenberg number. The length distribution is the same for all of the results and the mean relaxation time is a constant. In the experiments, the Weissenberg number is only adjusted by 
changing the flow rate. Therefore, we predominately use the shear rate $\dot{\gamma}$ as the parameter to characterize the flow and not the Weissenberg number. To obtain a rough idea of the Weissenberg number, the shear rate needs only to be multiplied by a relaxation time of $\tau_{\text {rel }} \approx 0.3-0.4 \mathrm{~s}$.

\subsection{Conformations of Actin Filaments}

\subsubsection{Orientation}
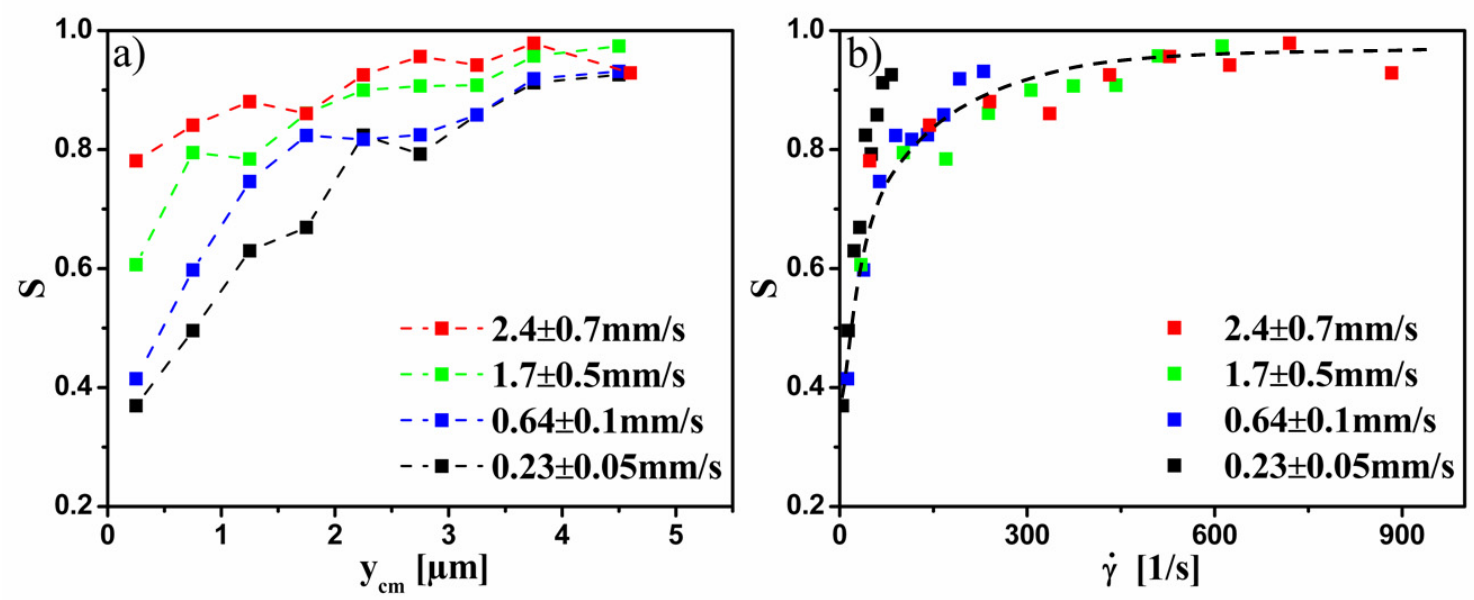

Figure 5-7: Orientational order parameter. a) Orientational order parameter plotted against the channel position. b) Orientational order parameter plotted against the shear rate. The dashed curve is only a guide to the eye.

In figure 5-7a, the orientational order parameter $S$ (see equation 5.6), which describes the averaged alignment of the filaments, is plotted versus channel position $y_{c m}$ for different velocities. The order parameter $S$ increases from the channel center $\left(y_{c m}=0 \mu \mathrm{m}\right)$ out to the channel walls $\left(y_{c m}=5.5 \mu \mathrm{m}\right)$. Accordingly, actin filaments at the channel center are less aligned compared to those near walls, where the filaments are oriented nearly parallel to the walls. It is also seen, by comparing the curves for different velocities, that the order parameter increases with larger velocities. Thus, the filaments are more aligned at higher flow rates. In Poiseuille flow, the local shear rate is positiondependent (see equation 5.2). At the channel center, the local shear rate is zero, and the influence of the flow on filament orientation is small. Close to the walls, the shear rate is large, and the flow affects the orientation of the filaments clearly. Hence, the increase in alignment of the filaments toward the walls might be caused by an increase of the local shear rates. Furthermore, the local shear rate at a certain channel position increases 
also with larger velocities. A consequence is an increase of alignment at identical positions for larger velocities, which is consistent with the observations.

By calculating the local shear rate from channel position and velocity (see equation 5.2), the direct relation between the order parameter and the shear rate is obtained and is shown in figure 5-7b. As expected, the averaged alignment of the filaments increases with larger shear rates. The curves for flow velocity $v_{0} \geq 0.64 \mathrm{~mm} / \mathrm{s}$ collapse in one single curve. This indicates that the different local shear rates are the fundamental cause for the position-dependent alignments of filaments inside the microchannel. Near walls, alignement is additionally influenced by the walls. This is in particular important for small velocities, where Brownian fluctuations of the shape are less suppressed. The deviations of several data points measured for the smallest velocity $v_{0} \approx 0.23 \mathrm{~mm} / \mathrm{s}$ from the master curve are probably generated by the walls.

So far, we described the influence of flow on filament orientation by the local shear rate at the center-of-mass of the filaments, which can be regarded as an averaged shear rate acting on the entire filament. However, this description predicts that filaments at the centerline ( $\dot{\gamma}=0$ ) should be not aligned (order parameter $S=0$ ), which is not the case in the measurements (see figure 5-7). Parts of the filaments always feel the shear influencing the conformation and orientation due to the filament extension perpendicular to flow direction that is not insignificant. So far, we ignored the microscopic picture of the polymer, where each polymer segment is influenced by the local shear rate at its own position - which can be slightly different from the shear rate at the center-of-mass. Except close to the walls, this description works quite well, confirmed by the collapse of the curves of the order parameter measured for different velocities onto a single curve depending only on shear rate at the center-of-mass. However, one feature of the Poiseuille flow is the non-monotonic behavior of the shear rate across the channel with a minimum $\dot{\gamma}=0$ at the centerline. In addition, filaments near the centerline, where small shear barely suppresses fluctuations, have a large extension perpendicular to the flow direction. Therefore, it is reasonable to expect that conformational changes of filaments crossing the centerline are more complicated than merely elongation and alignment in the flow direction. A more detailed analysis of these filaments near the centerline is given in section 5.4.3.

The order parameter at a certain channel position (e.g. $y=2.5 \mu \mathrm{m}$ ) does not dramatically change with velocity. However, the order parameter describes only an averaged alignment of filaments in flow. The orientations of filaments at one channel position can be characterized in more detail by the probability distribution of the angle $\Theta$. The angle probability distribution is obtained by dividing the $\Theta$-scale in intervals of $\Delta \Theta=2^{\circ}$ and counting for each interval the number of filaments with corresponding angles. The 
distribution is normalized by the total number of analyzed filaments. In figure 5-8, angle probability distributions are shown for filaments located at $y_{c m}=(2.5 \pm 1) \mu \mathrm{m}$ for the different flow velocities. The channel position $y_{c m}=(2.5 \pm 1) \mu \mathrm{m}$ is almost at the halfway point between the channel walls and the channel center. This position is chosen because effects due to the non-monotone behavior of the local shear rate at the channel center can be negligible as well as effects due to confining walls.

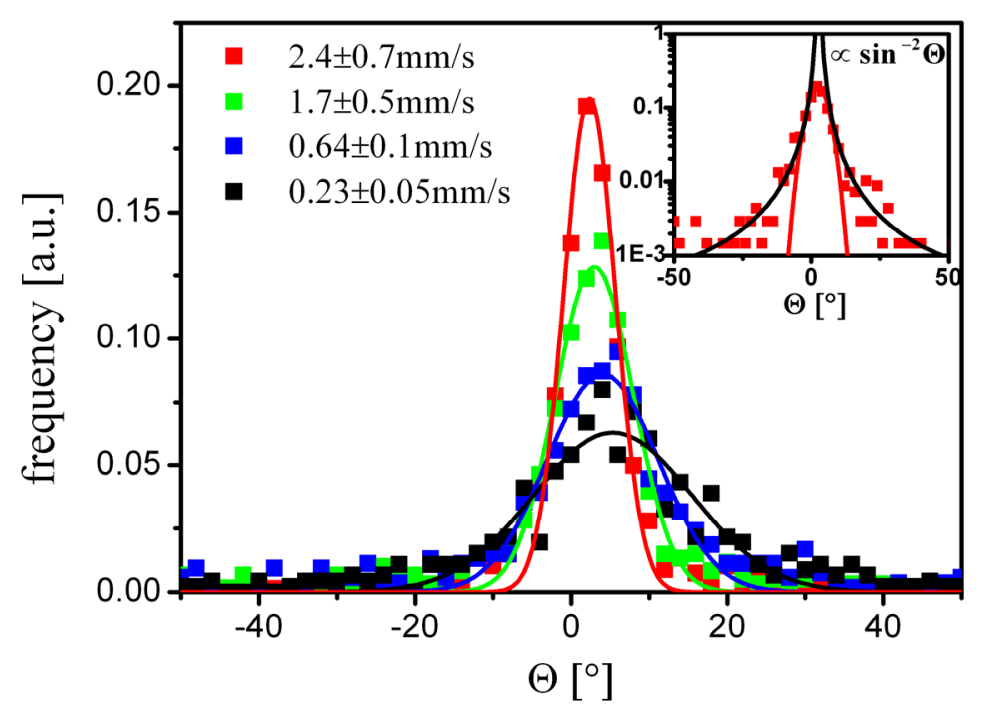

Figure 5-8: Angle probability distribution of actin filaments at $y_{c m}=2.5 \pm 1 \mu \mathrm{m}$ for different velocities. Lines are Gaussian fits. The corresponding local shear rates at the position $y_{c m}=2.5 \mu \mathrm{m}$ are $480 \mathrm{~s}^{-1}$, $340 \mathrm{~s}^{-1}, 130 \mathrm{~s}^{-1}$ and $50 \mathrm{~s}^{-1}$. The inset shows the distribution in a semi-logarithmic scale for the largest velocity $v_{0}=2.4 \pm 0.7 \mathrm{~mm} / \mathrm{s}$.

The angle probability distributions for the different velocities all have the same characteristics. The distributions are almost symmetric with respect to the maximum. With increasing velocities, the distribution narrows and the absolute value of the maximum increases. Thus, the filaments align almost entirely in the flow direction and the alignment increases with velocity - as already expected from the previous discussion of the order parameter. Indeed, the position $\Theta_{t}$ of the maximum is slightly above $\Theta=0$ for all velocities, and shifts to lower angles with increasing velocity. Consequently, the preferred orientation of the actin filaments is slightly larger than zero but converges to the scenario in which the filaments are perfectly aligned parallel to the walls.

It can be seen in figure 5-8 that the angle probability distributions can be fitted as Gaussian distributions. For flexible polymers in simple shear flow, it has been reported that at larger Weissenberg numbers only the tails of the distributions deviate strongly from Gaussian distributions [20]. Some theories predict a weaker decay with a scaling of $\Theta \propto \sin ^{-2} \Theta$ for $\mid \Theta>>\Theta_{t}$ at large Weissenberg numbers [95, 96]. It is seen in the 
inset in figure 5-8 (semi-logarithmic plot), that this scaling describes the tails in the distribution for a large velocity $v_{0}=2.4 \pm 0.7 \mathrm{~mm} / \mathrm{s}$ quite well.
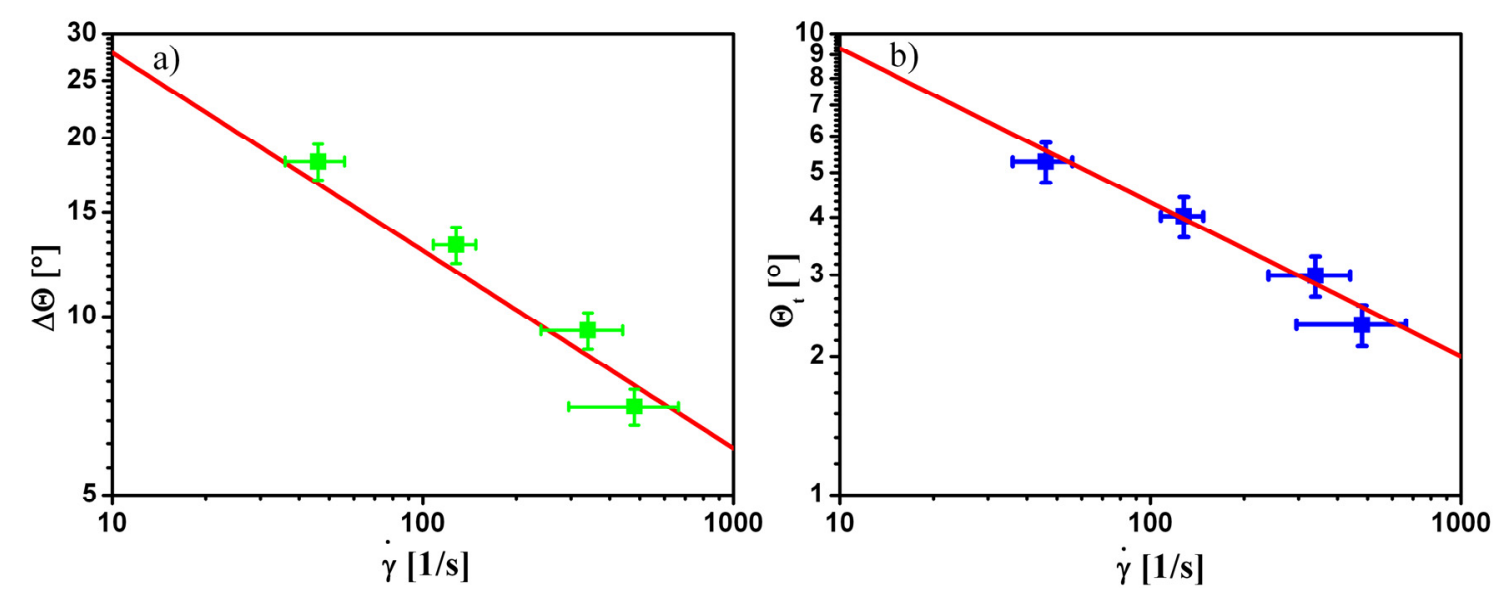

Figure 5-9: a) Width $\Delta \Theta$ of the angle probability distributions. b) Position $\Theta_{t}$ of the maximum of the angle probability distributions. Red lines have the slope of $-1 / 3$.

The width $\Delta \Theta$ and the maximum position $\Theta_{t}$ of the angle probability distributions, obtained from the Gaussian fits, are shown in figure 5-9. The flow field at the channel position of interest can be roughly described as simple shear flow (see [20]) with shear rate $\dot{\gamma}(2.5 \mu \mathrm{m})$. For less elongated flexible polymers $(R<<L)$ scaling laws $\Theta_{t} \propto \Delta \Theta \propto W i^{-1} \propto \dot{\gamma}^{-1}$ (see equation 4.41) are predicted, which are quite different to scaling laws $\Theta_{t} \propto \Delta \Theta \propto W i^{-1 / 3} \propto \dot{\gamma}^{-1 / 3}$ (see equation 4.42) predicted for strongly elongated or stiff polymers $(R \approx L)$ at large Weissenberg numbers. The red lines in the double logarithmic plots in figure 5-9 have a slope of $-1 / 3$. Comparison of these lines with the experimental data points shows that the width and the maximum position can be described with the scalings of elongated polymers. Therefore, the actin filaments behave more like a rigid rod than an elastic flexible polymer concerning their orientation. In contrast, in experiments for flexible polymers in simple shear flow a cross-over region has been observed between a decay with slope of -1 for small Weissenberg numbers and a decay with slope of $-1 / 3$ for large Weissenberg numbers $[20,129]$. However, actin is a semiflexible polymer. In contrast to flexible polymers like DNA, which are coiled in equilibrium $(R<<L)$, actin filaments already have an extended configuration $(R \leq L)$ without flow. Therefore, it is not surprising that all data points can be described by a single scaling law.

As noted above, the angle probability distributions are not symmetric with respect to $\Theta=0$. Rather, the entire curves are shifted to a value slightly larger than zero. In figure $5-10$, two different scenarios for the filament orientation depending on the sign of the 


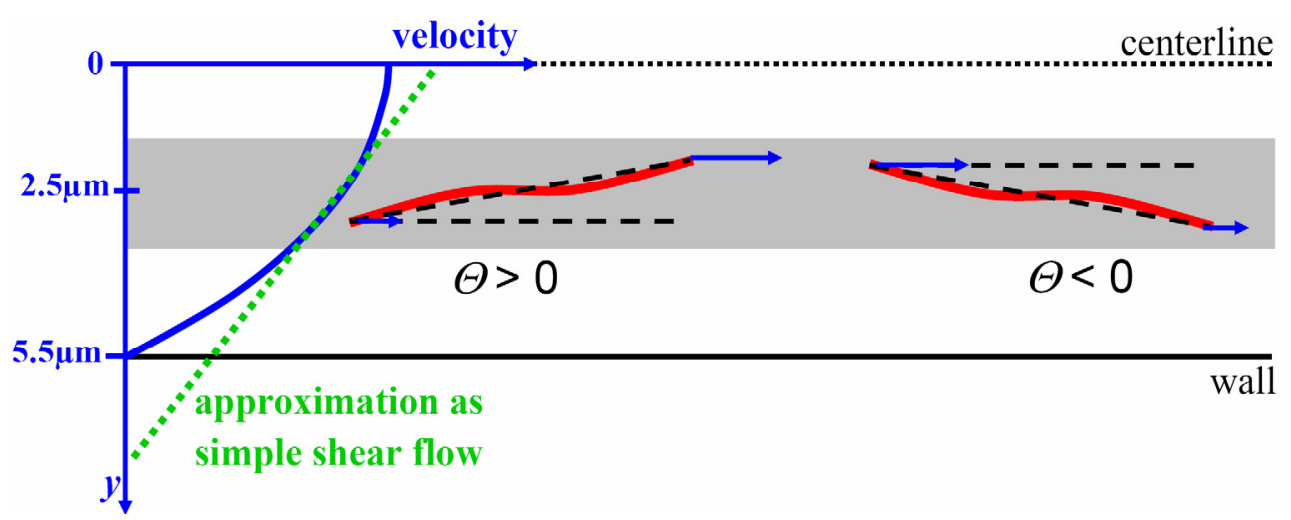

Figure 5-10: Scenarios for filaments with a positive angle and for filaments with a negative angle.

angle are shown. The two scenarios are quite different concerning their velocity at the end-points of the filament. For an angle $\Theta>0$, the filament is in a stable conformation. Depending on the shear rate, the filament will be elongated a little more, but the basic elongated shape will persist. On the other hand, thermal fluctuations can move the filament in a scenario with $\Theta<0$. In this case, the actin filament has an unstable conformation and the end with the faster velocity will pass the other end. In fact, we have observed such tumbling in experiments with actin filaments (figure 5-3c). The tumbling of the filament will continue until a stable conformation is obtained. Consequently, the probability to find filaments with $\Theta>0$ is larger, and this explains the preferred positive values in the angle probability distribution.

\subsubsection{Elongation and Tumbling}

As seen already in the snapshots of the actin filaments in figure 5-3, the actin filaments change their conformations in flow. To quantify the conformational changes for a certain channel position $y_{c m}=(2.5 \pm 1) \mu \mathrm{m}$, the end-to-end distance $R$ normalized by the contour length $L$ of each filament is measured. The end-to-end distance probability distribution is obtained by dividing the $R / L$-scale in intervals of $\Delta R / L=0.02$ for which the numbers of filaments with corresponding $R / L$ are counted. The distribution is normalized by the total number of analyzed filaments. In figure 5-11a, the end-to-end distance probability distributions are shown for the no flow scenario and for two flow velocities. Without flow, filaments with a normalized end-to-end distance $R / L<0.7$ are not found. The number of filaments with a certain $R / L$ increases slowly for larger $R / L$ values until the maximum $R / L \approx 0.97$ is reached and the distribution shows a steep decrease. This end-to-end distance probability distribution of actin filaments in microchannels without flow is approximately in agreement with theoretical calculations 

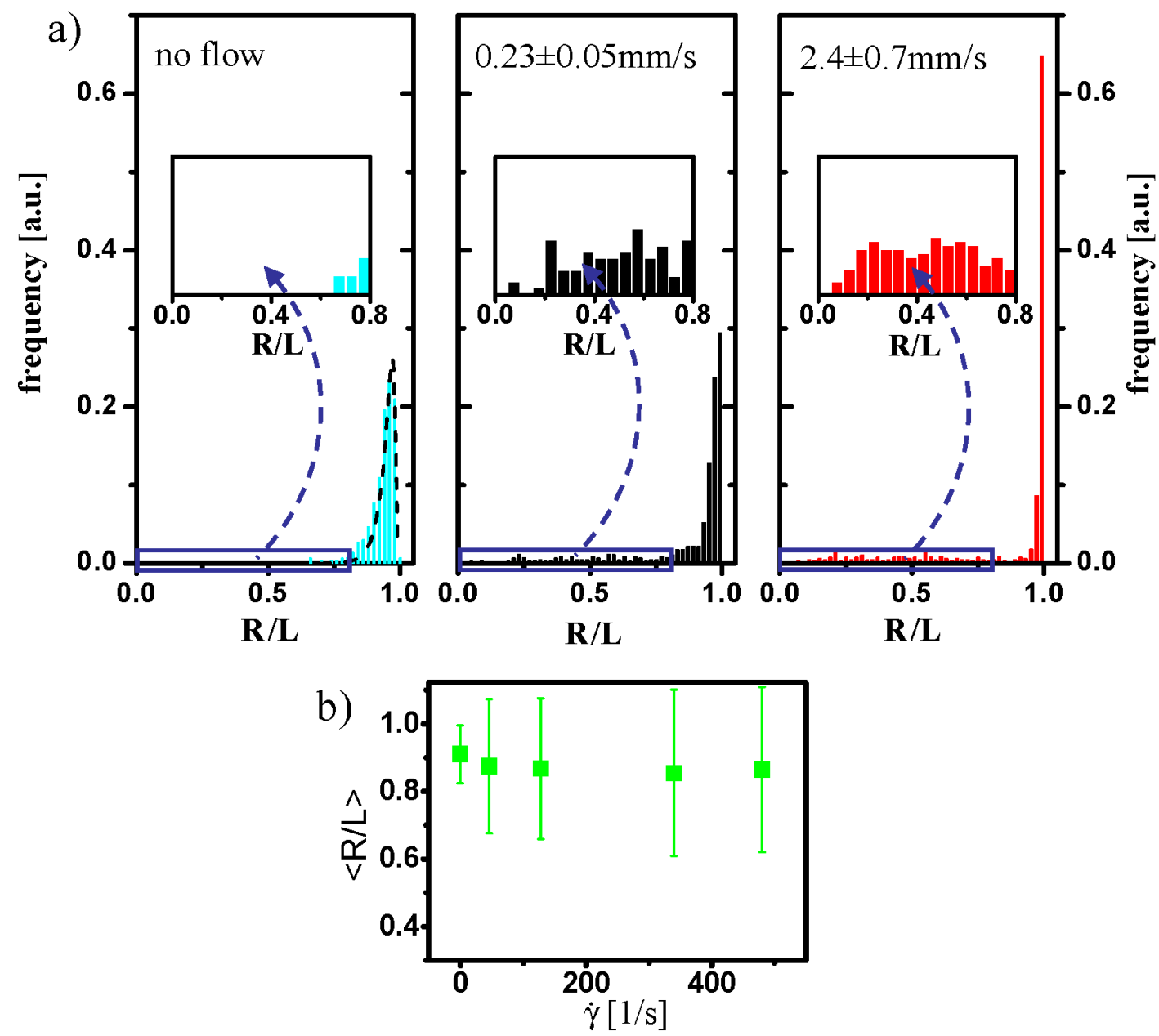

Figure 5-11: Normalized end-to-end-distances of actin filaments in flow. a) End-to-end distance probability distributions for different velocities. The line in the plot for the no flow scenario is the theoretical expectation (equation 3.5) of a worm-like chain with $L_{p}=13 \mu \mathrm{m}$ and $L=8 \mu \mathrm{m}$. Insets shows the end-to-end-distance probability distributions of small $R / L$ values for which the $R / L$-scale was subdivided in larger intervals of $\Delta R / L=0.05$. The corresponding local shear rates at the position $y_{c m}=2.5 \mu \mathrm{m}$ are $0 \mathrm{~s}^{-1}, 50 \mathrm{~s}^{-1}$ and $480 \mathrm{~s}^{-1}$. b) Averaged end-to-end distance $<R / L>$ against shear rate (error bars: standard deviation).

for modeling the filament as worm-like chain with persistence length $L_{p}=13 \mu \mathrm{m}$ and contour length $L=8 \mu \mathrm{m}$ (see line in figure 5-11). When flow is added, the maximum of the distribution becomes larger, shifts to larger $R / L$ values and the distribution narrows. In other words, the filaments are more elongated in flow. Furthermore, it is seen in the insets in figure 5-11a, by comparing the distributions for small $R / L$ values, that more actin filaments with smaller $R / L$ exist in flow than in equilibrium. These values belong to the tumbling filaments which are mentioned already in the last section. During tumbling, the ends of the filaments come close together, and the end-to-end distance of actin filament in flow is sometimes even smaller than in equilibrium. The elongation 
and the tumbling of the filaments are considered in the following discussions in more detail.

Typically, the degree of elongation is described in polymer science by the averaged endto-end distance [89], and therewith the behavior of DNA in shear flow, for example, has been characterized [129]. However, calculating the average of all measured normalized end-to-end distances, a decrease of $\langle R / L>$ for actin filaments in flow is obtained that is seen in figure $5-11 \mathrm{~b}$. The cause of this decrease is the tumbling of filaments, although about $70 \%$ of all analyzed filaments are in an elongated state. Therefore, the averaged end-to-end distance is not the correct parameter to characterize the elongation of the filaments in flow. Without flow, the measured averaged end-to-end distance $<R / L>=0.91$ is consistent with theoretical expectations (see equation 3.4) of $<R / L>=0.90$ for actin filaments modeled as worm-like chain with persistence length $L_{p}=13 \mu \mathrm{m}$ and contour length $L=8 \mu \mathrm{m}$.

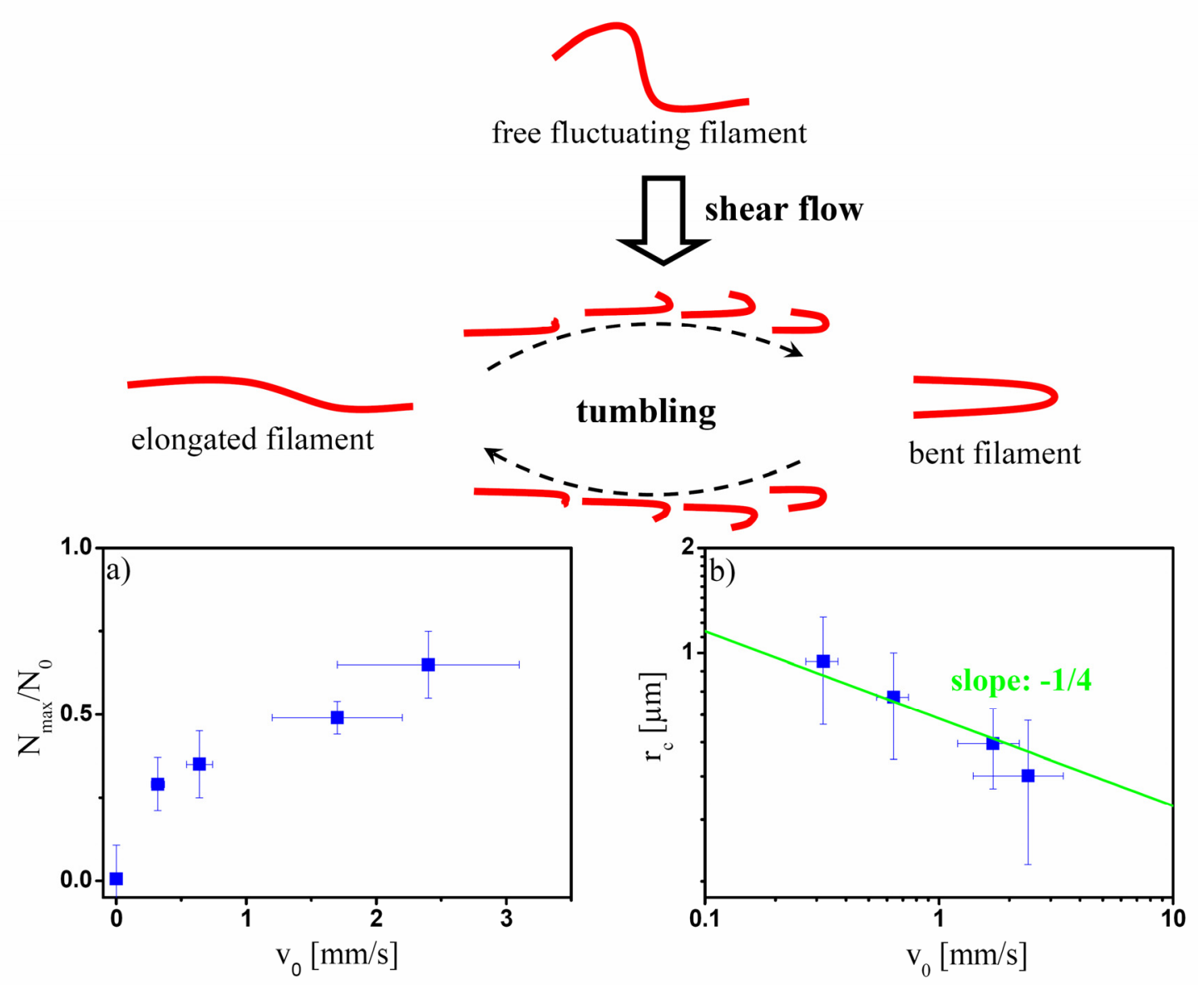

Figure 5-12: Conformations of actin filaments in flow. Flow elongates the semiflexible polymer. Due to thermal fluctuations of the shape, a filament can move in an unstable scenario $(\Theta<0)$ and the filament tumbles. a) Number of fully-elongated filaments against velocity. b) Radius of curvature of bent filaments during tumbling. 
Using the definition that filaments have a fully elongated state for $R / L>0.9$, the degree of elongation with larger flow velocities can be quantified by the number of fully elongated filaments $N_{\max }$ relative to the total number $N_{0}$ of analyzed filaments (see figure 5-12a). $N_{\max } / N_{0}$ increases with larger velocities as progressively more filaments become fully elongated. But $N_{\max } / N_{0}$ is clearly below one for all flow velocities, which would be the case if all filaments were fully elongated. Fully elongated filaments lie on a single streamline, where no force to elongate the filaments exists because all parts of a filament have the same velocity. The absence of the elongational force causes relaxation. For relaxed filaments, the velocity along the contour varies, the drag forces at the segments are different, and the filaments will be elongated again. Therefore, filaments in flow stretch and relax constantly and the state of full elongation for all filaments can never be obtained, not even at very large shear rates. In addition, the relaxed filaments, for which the thermal shape fluctuations are large, tumble sometimes instead of elongate, depending on their orientations relative to the flow direction (see section 5.4.1).

Tumbling of polymers has been also observed for flexible polymers such as DNA in shear flow [129, 130]. During tumbling, flexible polymers choose many different conformational pathways, and they often relax back into a coiled state. In contrast, the conformations of actin filaments are always similar in their observed tumbling motions and the actin filaments are more strongly bent than in equilibrium. The semiflexible actin filaments resist the flow field, which tries to fold the polymer, and the bent shapes with always the same finite curvature reflects the large persistence length of the semiflexible polymer. The bent contour of the tumbling filaments can be approximated by a constant radius of curvature (see figure 5-13b). The averaged radius of curvature $r_{c}$ is plotted against the velocity in figure $5-12 b$. With larger velocities, the radius of curvature decreases and the filaments are more strongly bent.

For a first analytical treatment [131], the filament is modeled as an elastic rod, which is bent along a semi-circle of radius $r_{c}$ with one long elongated end (see figure 5-13a). Comparable shapes are found experimentally (see figure 5-13b) in early states during tumbling. The bending for later tumbling states can be described analogously, resulting in the same scaling argument.

Parameterization of the semi-circle in polar coordinates with the arc length $s=r_{c} \varphi$ results in

$$
\mathbf{r}=\left(\begin{array}{c}
\tilde{x} \\
\tilde{y}
\end{array}\right)=\left(\begin{array}{c}
-r_{c} \sin \left(s / r_{c}\right) \\
r_{c} \cos \left(s / r_{c}\right)
\end{array}\right)
$$




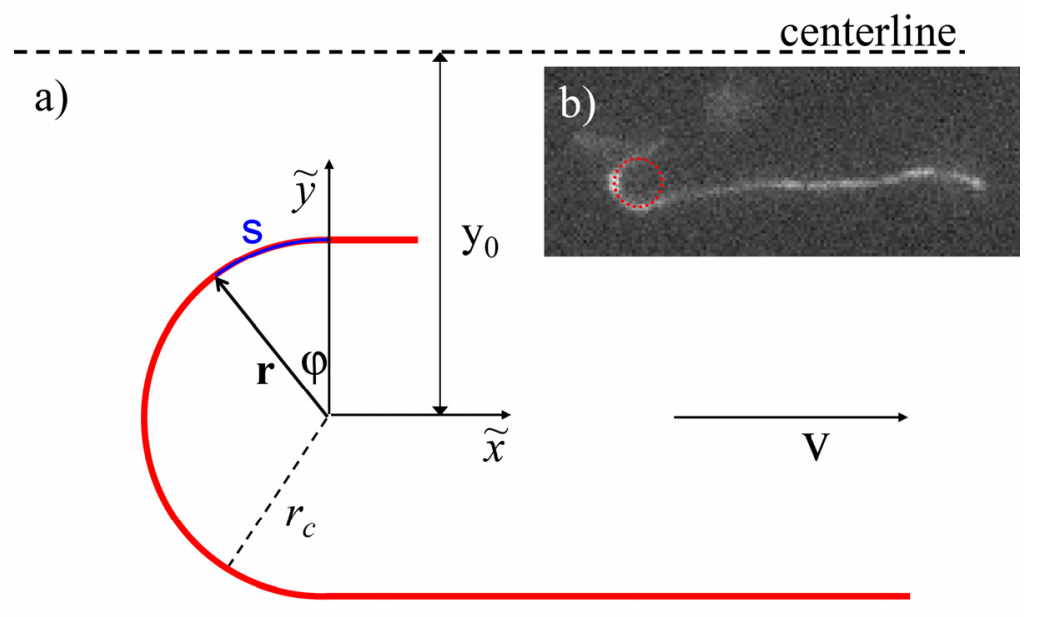

wall

Figure 5-13: Radius of curvature of tumbling filaments. a) Model of a bent filament during tumbling. The curvature of the contour is assumed to be constant with radius of curvature $r_{c}$. The filament is considered at a channel position $y_{0}$. b) Snapshot of an actin filament during tumbling.

The hydrodynamic beam equation of an elastic rod connects the drag force, which induces bending, to the bending moment of an elastic rod [81]:

$$
\left|\frac{\partial^{4} \mathbf{r}}{\partial s^{4}}\right|=-\frac{1}{\kappa} c_{f} v,
$$

where $\kappa$ is the bending rigidity (see equation 3.1), $c_{f}$ is the friction coefficient per length, and $v$ is the velocity of the rod relative to the solvent.

The movement of the ends of the filament relative to each other during tumbling is much slower than for two solvent molecules with the same starting positions. It is therefore justifiable to assume a constant velocity of the entire filament at one snapshot during tumbling and to approximate the filament velocity by the solvent velocity $v\left(y_{0}+r_{c}\right)$ at the long end of the filament (where most of the filament still is). The solvent velocity at the channel position of interest is given by the velocity profile of the Poiseuille flow (see equation 5.1). The averaged velocity of the filament relative to the solvent can be estimated as the filament velocity $v\left(y_{0}+r_{c}\right)$ relative to the solvent velocity $v\left(y_{0}\right)$ at the position $y=y_{0}$ :

$$
v\left(y_{0}+r_{c}\right)-v\left(y_{0}\right)=-\frac{v_{0}}{(d / 2)^{2}}\left(2 y_{0} r_{c}+r_{c}^{2}\right) .
$$

Considering the tumbling of a filament at a position $y_{0}=2.5 \mu \mathrm{m}$, the first term on the right side is larger than the second term, because typical radii of curvature in experiments are $r_{c}<1 \mu \mathrm{m}$ (see figure 5-12b). Therefore, the averaged velocity of the 
filament relative to the solvent can roughly be described as

$$
v\left(y_{0}+r_{c}\right)-v\left(y_{0}\right) \approx-\frac{2 v_{0}}{(d / 2)^{2}} y_{0} r .
$$

With this estimation and equation 5.9, the hydrodynamic beam equation (equation 5.10) results in

$$
\frac{k_{b} T L_{p}}{r_{c}^{3}} \approx 2 v_{0} c_{f} \frac{r_{c} y_{0}}{(d / 2)^{2}} .
$$

Thus, the dependence of the radius of curvature on the velocity $v_{0}$ is given by

$$
r_{c} \approx\left(\frac{k_{b} T L_{p} d^{2}}{8 v_{0} c_{f} y_{0}}\right)^{1 / 4} \propto v_{0}^{-1 / 4} .
$$

This scaling is consistent with the experimental values, which is seen in the double logarithmic plot in figure 5-12b. The measured data points are well described by the green line, which has a slope of $-1 / 4$. Thus, the actin filaments behave like elastic rods that are bent due to different drag forces along the contour and balanced by the bending forces.

In summary, actin filaments in pressure-driven flow are elongated or tumble. An exception is found at the channel center, where the behavior is quite different and is discussed in the next section. The averaged alignment in flow direction is positiondependent and increases toward the walls as well as for larger velocities. The orientation of a filament decides whether the filament will elongate or will tumble. Most of the time, an actin filament is elongated and the elongation is on average larger for increased velocities. For a fully-elongated filament, the elongational force is missing and the filament will relax. For a relaxed filament, the thermal shape fluctuations are large. The fluctuating filament elongates again or moves in an unfavorable orientation and tumbles. A tumbling filament can be modeled as an elastic rod for which the radius of curvature decreases with larger velocities. After finishing the tumbling cycle, the filament will elongate or tumble once more, depending again on the orientation. Thus, the actin filaments change constantly their shapes and elongate, relax and tumble.

\subsubsection{Conformations at the Centerline}

Figure 5-14 shows actin filaments near the centerline. In this region, the actin filaments can be divided into two classes: elongated filaments (figure 5-14a) and bent filaments (figure 5-14b). Filaments also exist which have an elongated end and the other end is bent (figure 5-14c). Elongated filaments rarely cross the centerline, and it seems that as soon as a filament crosses the centerline it becomes bent. 


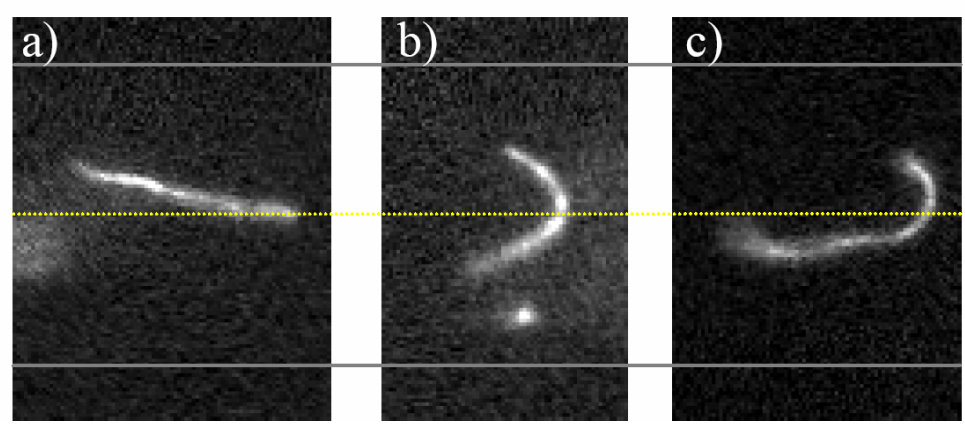

Figure 5-14: Conformations of actin filaments near the centerline (yellow line: centerline, gray lines: walls) for $v_{0}=1.7 \pm 0.5 \mathrm{~mm} / \mathrm{s}$.

Whereas elongated filaments near the centerline are in accordance with elongated filaments at $y=2.5 \mu \mathrm{m}$ considered in the previous section, bent filaments at the centerline differ from the bent filaments during tumbling. In contrast to the tumbling filaments, bent shapes at the centerline are relatively stable. The evolution of a bent filament can be seen for instance in figure 5-3c.

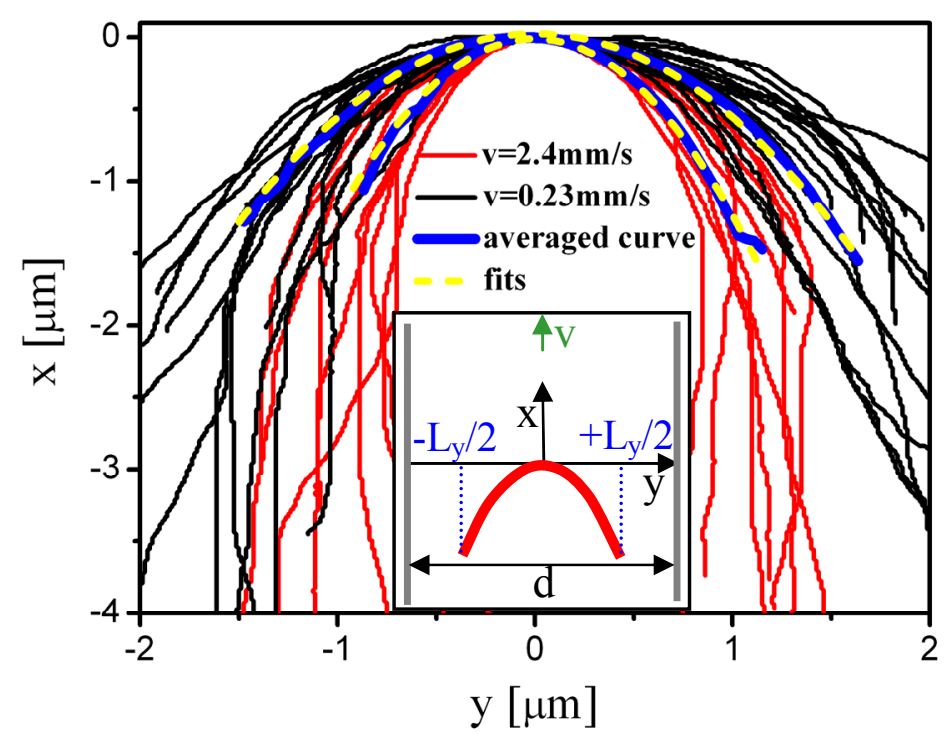

Figure 5-15: Bent filaments at the centerline. The red and black lines represent individual filaments at a velocity of $v_{0}=2.4 \pm 0.7 \mathrm{~mm} / \mathrm{s}$ and $v_{0}=0.23 \pm 0.05 \mathrm{~mm} / \mathrm{s}$, respectively. The blue lines show the average of all curves of individual filaments having the same velocity. The yellow lines are parabolic fits of the averaged curves.

In figure 5-15, the $x-y$ coordinates of bent filaments are plotted for two velocities. The filaments are more strongly bent for the larger velocity. The blue lines are averaged curves, and the yellow lines are the parabolic fits of these averaged curves. Therefore, 
the experimentally determined shapes of the filaments can be described with parabolic profiles, at least for the parts of the contours close to the centerline. The velocity field of a Poiseuille flow is also parabolic (equation 5.1), and it seems that the bent parabolic shapes reflect characteristics of the flow field. In the following discussion, we seek a connection between the bent filaments and the parabolic velocity field.

To analyze the bending of actin filaments in the middle of the channel and its connection to the parabolic velocity field, actin filaments are considered as elastic rods (see inset in figure 5-15) [131]. With such a simple polymer model, the bending during tumbling is already described successfully (section 5.4.2). For simplification, we consider only symmetric filaments with ends at $y= \pm L_{y} / 2$. The shape of the rod is determined by the balance of drag force and bending force at each point of the rod, neglecting Brownian forces in a first consideration. This leads to the hydrodynamic beam equation (equation 5.10), which has already been used to studying the bending of tumbling filaments. In Cartesian coordinates and small angle approximation, the hydrodynamic beam equation is given by [81]

$$
c_{f} \frac{\partial x}{\partial t}=-k_{B} T L_{P} \frac{\partial^{4} x}{\partial y^{4}},
$$

where the velocity $\partial x / \partial t$ is related to the solvent velocity. We want to describe stable conformations and therefore the velocity $v_{p}$ at each point of the filament is the same. Assuming a parabolic flow profile for the solvent, the velocity $\partial x / \partial t$ results in $\partial x / \partial t=v_{p}-v_{0}\left(1-y^{2} /(d / 2)^{2}\right)$ and the hydrodynamic beam equation can be written as

$$
v_{p}-v_{0}\left(1-\frac{y^{2}}{(d / 2)^{2}}\right)=-\frac{k_{B} T L_{P}}{c_{f}} \frac{\partial^{4} x}{\partial y^{4}} .
$$

To solve this differential equation, a polynomial ansatz is made

$$
x(y)=\frac{c_{2}}{2} y^{2}+\frac{c_{4}}{24} y^{4}+\frac{c_{6}}{720} y^{6},
$$

which already takes into account that all coefficients of power higher than 6 are zero, and all coefficients of uneven power have to be zero due to the symmetry of the equation. With this ansatz, the coefficients

$$
\begin{aligned}
c_{4} & =-\frac{c_{f}}{k_{B} T L_{P}}\left(v_{p}-v_{0}\right) \\
c_{6} & =-\frac{4 c_{f} v_{0}}{k_{B} T L_{P} d^{2}}
\end{aligned}
$$

can be determined. 
Taking as a boundary condition that there is no force or torque at the free ends of the filament

$$
\begin{aligned}
& \left.\frac{\partial^{2} x(y)}{\partial y^{2}}\right|_{y= \pm L_{y} / 2}=0 \\
& \left.\frac{\partial^{3} x(y)}{\partial y^{3}}\right|_{y= \pm L_{y} / 2}=0
\end{aligned}
$$

the velocity $v_{p}$ of the filament is given by

$$
v_{p}=v_{0}\left(1-\frac{L_{y}^{2}}{3 d^{2}}\right)
$$

and the coefficient $c_{2}$ can be calculated to

$$
c_{2}=-\frac{c_{f} v_{0}}{48 k_{B} T L_{P} d^{2}} L_{y}^{4} .
$$

With all calculated coefficients and knowledge of the filament velocity, the shape of a bent filament in the middle of a channel is given by

$$
x(y)=-\frac{L_{y}^{4}}{96 l^{3} d^{2}} y^{2}+\frac{L_{y}^{2}}{72 l^{3} d^{2}} y^{4}-\frac{1}{90 l^{3} d^{2}} y^{6},
$$

where $l=\left(k_{B} T L_{P} /\left(c_{f} v_{0}\right)\right)^{1 / 3}$. Thus, the shape of filaments at the centerline can be considered as a parabolic shape with some higher ordered correction terms. This is in excellent agreement with experiment, where we indeed often found parabolic profiles of bent filaments in the channel center (see figure 5-15).

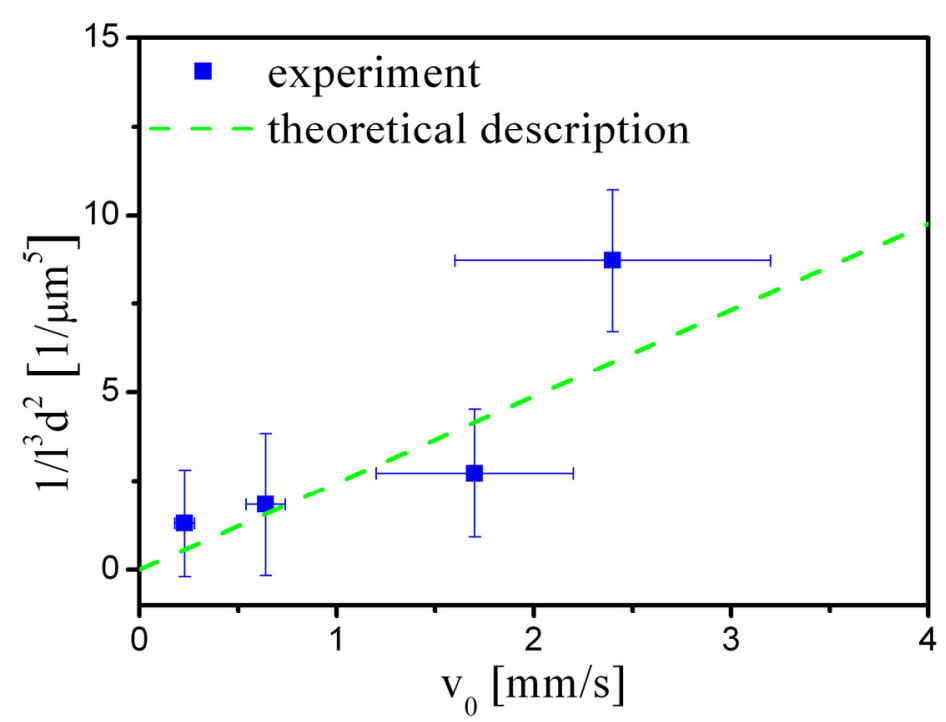

Figure 5-16: Comparison of the calculated prefactor of the parabolic profiles for filaments in the channel center with the experimental values. 
To check experimental and theoretical descriptions, the prefactor $1 / l^{3} d^{2}$ has to be compared. From the parameters of the parabolic fits of the averaged experimental curves we calculate $1 / l^{3} d^{2}$, with results given in figure 5-15. In this case, the length $L_{\mathrm{y}}$, which is needed for calculation, is obtained by assuming a symmetric filament with experimentally found parabolic shape and a contour length of $L=8 \mu \mathrm{m}$. We also calculated $1 / l^{3} d^{2}$ from polymer properties $\left(L_{p} \approx 13 \mu \mathrm{m} ; c_{f} \approx 1.8 \mathrm{mPas} / \mathrm{m}\right)$ and channel dimension $(d=11 \mu \mathrm{m})$. It is seen in figure 5-16 that the theoretical description is in reasonable agreement with the experimentally determined data points. Thus, with this simple model of an elastic rod we understand the existence of parabolic shapes at the centerline caused by the parabolic velocity field and we can describe them quantitatively. Brownian motion, which is neglected in this model, will lead to some fluctuations of the basic parabolic shape but is nevertheless not required in this model.

\subsection{Center-of-Mass Probability Distribution}

After analyzing the conformations and orientations of flowing actin filaments in microchannels, this section studies the probability to find a filament at a certain channel position. For this, the center-of-mass of each filament is measured and the numbers of filaments at certain channel positions $y_{c m}$ in intervals of $\Delta y=0.5 \mu \mathrm{m}$ along the crosssection are counted. The center-of-mass probability distributions are shown in figure 5-17 for different flow velocities. The distributions are normalized by the total number of analyzed filaments. An experimental uncertainty is caused by the 2-dimensional imaging of the filaments in the focal plane of the microscope (see section 5.1). Only filaments found mainly in the focal plane are included into the statistics. Due to this selection, filaments with unfavorable orientations relative to the focal plane are neglected in the statistics although they have their center-of-mass in the considered plane. The error bars in the plots of figure 5-17 are calculated by estimating the percentage of bent filaments with larger extension than $1 \mu \mathrm{m}$ in $z$-direction (perpendicular to the focal plane). For this error estimation, the bent filaments (parabolic bent filaments at the channel center and tumbling filaments) are considered because they have larger extensions perpendicular to the flow direction than the elongated filaments. Since the filaments are more bent at larger shear rates (see sections 5.4.2 and 5.4.3), the center-of-mass probability distributions become more precise toward the walls and at larger velocities. 

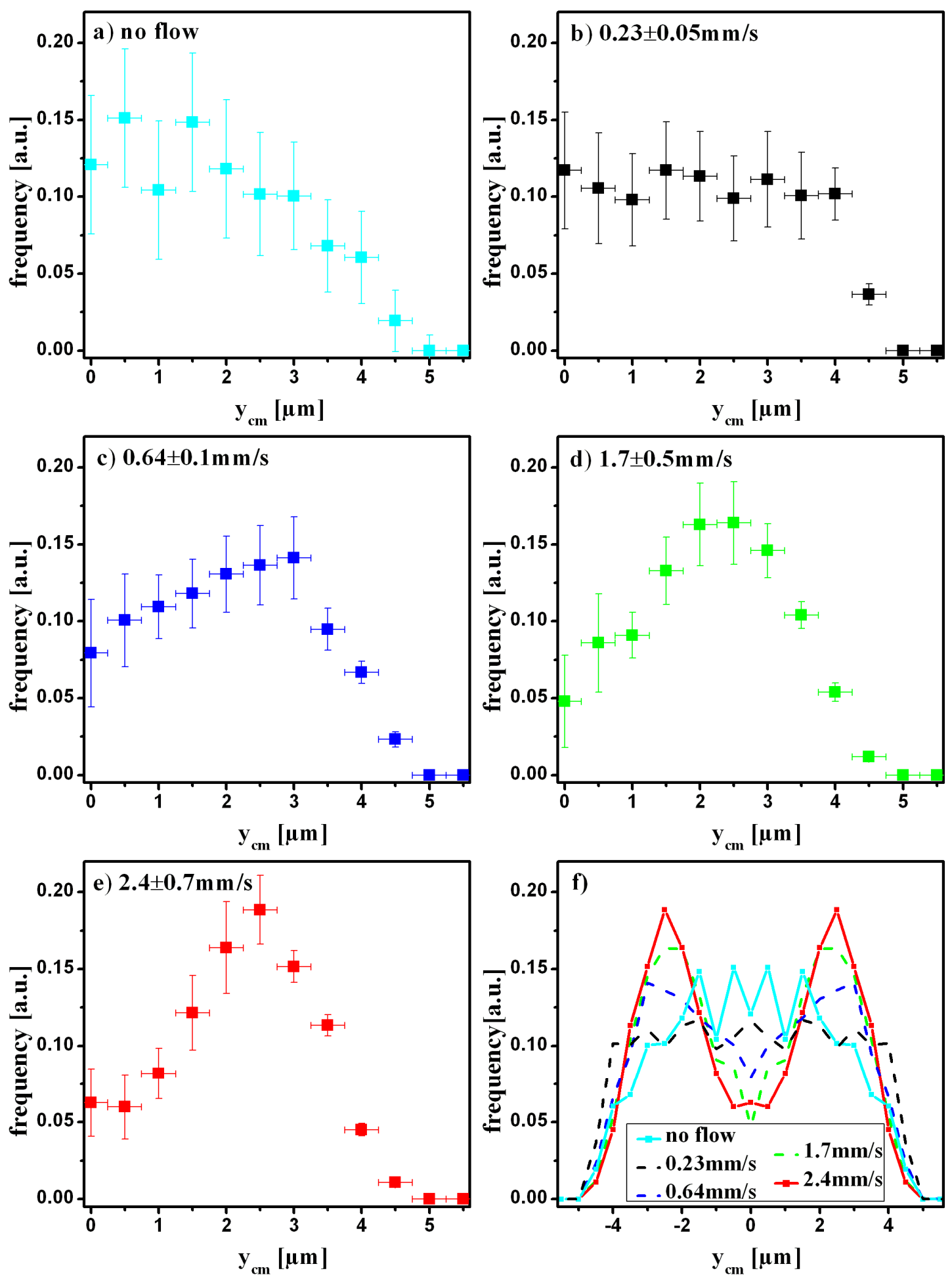

Figure 5-17: a)-e) Center-of-mass probability distributions for different flow velocities. The plots show the distribution of filaments along a cross-section of the channel for one channel half with the channel center at $y_{c m}=0 \mu \mathrm{m}$ and the channel wall at $y_{\mathrm{cm}}=5.5 \mu \mathrm{m}$. f) Comparison of the center-ofmass distributions for the different velocities. The plots show the distributions along the crosssection of the entire channel with walls at $y_{c m}=-5.5 \mu \mathrm{m}$ and $y_{c m}=5.5 \mu \mathrm{m}$ by mirroring the distributions seen in a)-e). 
Without flow, the center-of-mass probability distribution has a maximum at the channel center and decreases toward the walls. Therefore, actin filaments are most frequently found at the channel center. Close to the channel walls, a depletion layer can be observed, where filaments are hardly found. At a small flow velocity $v_{0}=0.23 \pm 0.05 \mathrm{~mm} / \mathrm{s}$, the distribution is constant almost over the entire channel and only very close to the walls a smaller number of actin filaments is still observed. In contrast, the center-of-mass probability distributions have a local minimum at the channel center for flow velocities $v_{0} \geq 0.64 \mathrm{~mm} / \mathrm{s}$. Most actin filaments in flow at large velocities are not found at the channel center, but approximately at the halfway point between the centerline and the walls. The minimum at the channel center becomes more pronounced with higher velocities. Furthermore, by comparing the distributions near the walls it can be seen that actin filaments are less depleted from the walls for a velocity $v_{0}=0.23 \pm 0.05 \mathrm{~mm} / \mathrm{s}$ than without flow, but an increase of the velocity leads again to larger depletion layers at the walls.

In recent computer simulations, the center-of-mass probability distributions of flexible polymers modeled by several connected bead-spring dumbbells have also shown a minimum at the channel center and depletion layers at the walls [29, 30, 121, 122, 132]. However, comparison of different simulations with comparable parameters points out some discrepancies concerning the height of the minimum as well as the size of the depletion layers, in particular for narrow channels. But it is quite striking to note that the minimum at the channel center is not pronounced in any of the simulations, even been discussed that this effect is negligible [132]. Simulations of Brownian stiff rods in Poiseuille flow predict also a minimum at the channel center but this minimum deviates only up to $10 \%$ from the maximal value $[31,123,124]$. Additionally, for stiff rods a depletion layer at the walls is also expected [123]. The results for the semiflexible actin filaments cannot be compared quantitatively with any of these simulations because other parameters are chosen and in particular the stiffness is not comparable. However, the strongly pronounced minimum at the channel center differs completely from the expectations of all simulations, hinting that this effect is caused by the semiflexible nature of the polymer.

Without flow, some configurations observed in free geometries are forbidden near a wall due to polymer-wall contacts. Therefore, the configurational entropy near a wall is reduced. This leads to a steric repulsion of polymers away from the walls and polymers are depleted in a layer which is in the order of the polymer size [23]. For stiff polymers, simulations [124] show that the center-of-mass probability distribution increases from zero at the wall to a constant value achieved at a half contour length away from the wall. A stiff polymer with a center-of-mass at this point can still touch the wall. For the 
semiflexible actin filaments, according arguments would predict that the steric hindrance affects the center-of-mass distribution near a wall up to half of the averaged end-to-end distance $\langle R / 2>$ away from the wall (see figure 5-18a). With an averaged end-to-end distance of about $7 \mu \mathrm{m}$ (equation 3.4), depletion is expected in a layer up to $3.5 \mu \mathrm{m}$ away from the walls which corresponds to a channel position $y_{c m} \approx 2 \mu \mathrm{m}$. This is in agreement with the measured center-of-mass probability distribution without flow. Aside from the constrained orientation near a wall, the semiflexible filaments are also sterically excluded due to their worm-like structure with extension perpendicular to the mean orientation of about a few micrometers.

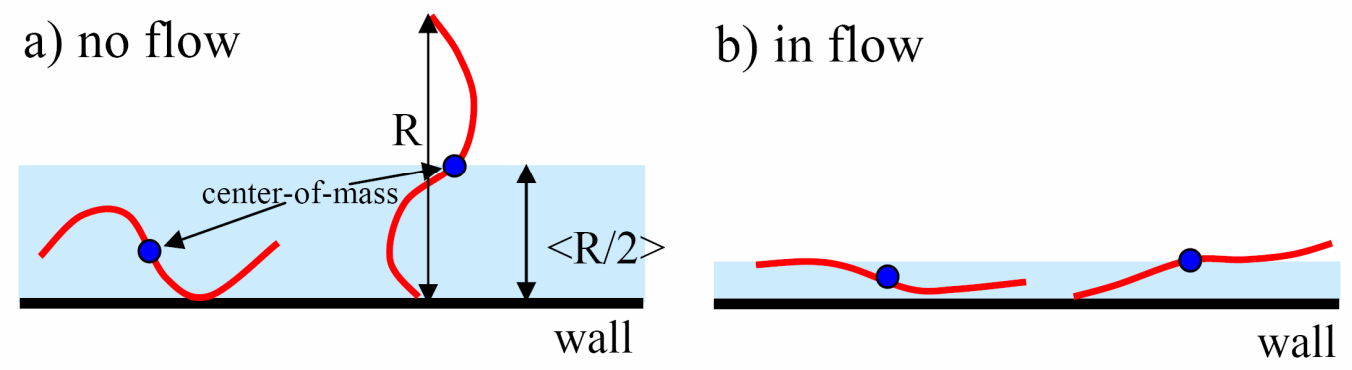

Figure 5-18: Steric volume exclusion. a) The orientations of the semiflexible filaments are randomly and the thermal fluctuations are large. This leads to a strong steric repulsion from the walls and large depletion layers. b) The filaments are aligned and elongated in flow. The steric hindrance is therefore reduced.

In flow, the filaments are elongated and aligned almost in flow direction (see section 5.4) and the steric hindrance of the filaments near the walls is decreased (see figure 5-18b). Consequently, depletion layers due to steric repulsion become smaller. This is consistent with the measured center-of-mass probability distribution, for which more actin filaments are found near the walls for a small velocity $v_{0}=0.23 \pm 0.05 \mathrm{~mm} / \mathrm{s}$ than for no flow conditions. Since the elongation and alignment of filaments increases with larger flow velocities, it could be expected that the depletion layer thickness decreases with increasing velocity. However, this is not the case and other effects, besides steric interactions, have to be discussed.

Generally, kinetic theories suggest two mechanisms leading to cross-streamline migration of flowing polymers in Poiseuille flow [26, 29]: hydrodynamic interactions with the walls cause migration of polymers away from the walls and spatially-varying diffusivity leads to migration away from the channel center. These two mechanisms are illustrated in chapter 4.4 by a dumbbell model and are summarized in figure 5-19. 

a) flexible polymer
b) semiflexible polymer
c) stiff polymer

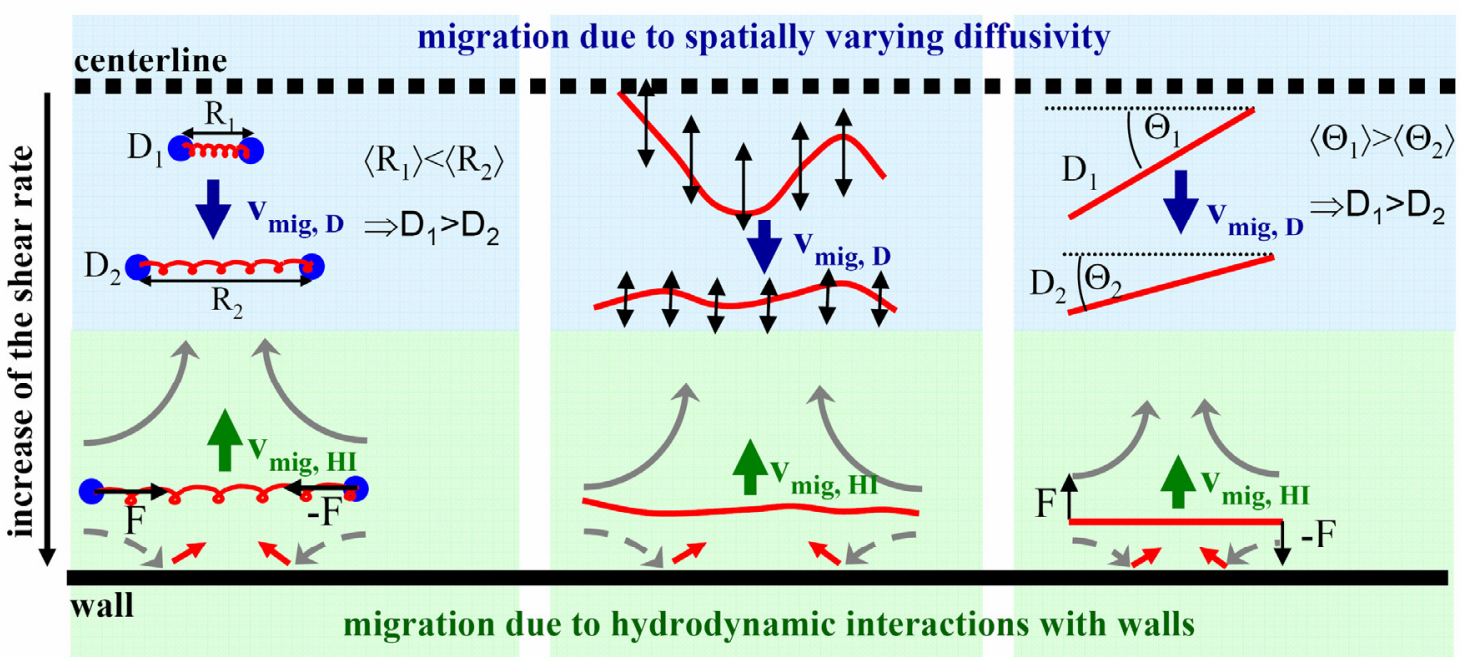

Figure 5-19: Migration of polymers. Relaxation of the polymer ( $\hat{=}$ recoil and/or reorientation of the polymer) induces an asymmetric microflow near the walls, which moves the polymer away from the wall. Different local shear rates inside the channel leads to position-dependent averaged stretching and/or orientation of the polymer. This leads to a spatially-varying diffusivity and to migration away from the centerline. a) Dumbbell model (see chapter 4.4). b) Semiflexible polymers. c) Brownian stiff rods.

Relaxation of the polymers, recoiling in the case of flexible polymers [25] and reorientation in the case of stiff polymers [123], induces an asymmetric microflow which moves the polymers away from the walls. An increase of the depletion layer thickness with higher velocities is expected such as it is also observed in the center-ofmass probability distributions for the semiflexible actin filaments. Therefore, it seems that the depletion layers are determined, additionally to steric interactions discussed above, by hydrodynamic interactions. Whereas the steric interactions are largest for the no flow scenario and decrease strongly in flow, the hydrodynamic interactions with the wall are zero without flow and become larger with higher velocities. Beside the wall effects, cross-streamline migration can be caused by spatially-varying diffusivity. In order that the diffusivity of a polymer depends on the channel position, the conformations have to vary inside the channel. In section 5.4, it is already shown that the conformations and orientations of the actin filaments change along the cross-section of the channel. Therefore, the precondition for a spatially-varying diffusivity is fulfilled. The diffusivity of the actin filaments inside the microchannel is discussed below in more detail. In general, migration away from the channel center is expected for polymers in Poiseuille flow [26, 29]. This leads to a local minimum in the center-of- 
mass probability distribution at the channel center such as it is observed for the actin filaments at large velocities. The maximum in the center-of-mass probability distributions of the actin filaments exists because filaments migrate away from the channel center, but also migrate away from the channel walls and accumulate therefore at a channel position between the channel center and the channel walls. Thus, kinetic arguments can explain qualitatively the center-of-mass probability distribution for the actin filaments in flow by steric interactions, hydrodynamic interactions with the walls, and spatially-varying diffusivity. Obviously, migration due to spatially-varying diffusivity dominates for the semiflexible actin filaments at large velocities and the attention is therefore mainly paid to the spatially-varying diffusivity in the following discussions.

\subsubsection{Analytic Description of the Center-of-Mass Probability}

\section{Distribution}

In the following section, we want to quantify the center-of-mass probability distributions of the semiflexible actin filaments by an analytic description. According to kinetic theories discussed above, an analytic description should include the drift due to hydrodynamic interactions with walls and the drift due to the spatially-varying diffusivity. Steric interactions are neglected for simplification. The evolution of the center-of-mass probability distribution $n\left(y_{c m}\right)$ along the cross-section of a straight channel is determined for any polymer in flow by

$$
\frac{\partial n}{\partial t}=-\frac{\partial}{\partial y_{c m}} j_{c}
$$

where the center-of-mass flux $j_{c}$ is given by

$$
j_{c}=K\left(y_{c m}\right) n-\frac{\partial D\left(y_{c m}\right)}{\partial y_{c m}} n-D\left(y_{c m}\right) \frac{\partial n}{\partial y_{c m}} .
$$

The first term in the flux describes the drift due to hydrodynamic interactions with the walls (an expression for $K\left(y_{c m}\right)$ is given below) [26], the second term describes the drift due to the spatially-varying diffusivity $D\left(y_{c m}\right)$ [32], and the last term is the contribution due to the Fickian diffusion. A derivation of this equation can be seen in chapter 4.4 for the dumbbell model (see equation 4.57). In steady-state, the flux must be zero. In order to solve equation 5.23 to obtain the center-of-mass probability distribution, the hydrodynamic interactions with the walls and the diffusivity of the actin filaments inside the microchannel have to be known. 


\subsubsection{Hydrodynamic Interactions with the Walls}

For only one single wall at $y=d / 2$, the drift of polymers due to hydrodynamic interactions with the wall is calculated in [26] and $K\left(y_{c m}\right)$ is derived to

$$
K\left(y_{c m}\right)=-\frac{\widetilde{K}\left(y_{c m}\right)}{\left(d / 2-y_{c m}\right)^{2}},
$$

where $\widetilde{K}\left(y_{c m}\right)$ depends on channel position as well as on polymer properties.

For semiflexible polymers, the dependence of $\widetilde{K}\left(y_{c m}\right)$ on the shear rate is given by [126]

$$
\widetilde{K}\left(y_{c m}\right) \propto \dot{\gamma}\left(y_{c m}\right)^{2 / 3} .
$$

With the relation between the shear rate and the channel position (equation 5.2), $\widetilde{K}\left(y_{c m}\right)$ can be written as

$$
\widetilde{K}\left(y_{c m}\right)=K_{0}\left(\frac{8 v_{0}}{d^{2}} y_{c m}\right)^{2 / 3},
$$

where $K_{0}$ is a proportional constant.

\subsubsection{Spatially-Varying Diffusivity}

In general, the dependence of the diffusivity on shape conformations of polymers is complicated and is also affected by hydrodynamic interactions [32, 33, 125]. For flexible polymers, the diffusivity changes mainly due to the elongation from coiled polymers at the channel center to stretched polymers with larger diffusivity at the walls (see figure 5-19a). The flexible polymers diffuse faster away than towards the channel center, and migration of polymers away from the channel center is the consequence. For stiff polymers, the flow influences strongly the averaged orientation of them within the channel (see figure 5-19c). Since the diffusivity of rods depends on the orientation (see equations 4.4 and 4.5 ), the diffusivity is also position-dependent for stiff rods but can only change maximal by a factor of two. For semiflexible actin filaments, the filaments elongate as well as orientate but the change in both parameters of filaments near the centerline compared to those near walls is rather small (see section 5.4.1). A particularity in the conformation of semiflexible actin filaments compared to flexible and stiff polymers with equal contour lengths is the large size of the thermal shape fluctuations perpendicular to the mean orientation of the polymer. Even in flow, the deviations of filament segments from the center-of-mass streamline are often several micrometers and therefore in the same order of magnitude as the channel width.

To measure these deviations, the contours of filaments are divided into segments in intervals of $\Delta x=0.5 \mu \mathrm{m}$ along the center-of-mass streamline (see figure 5-20). Since 


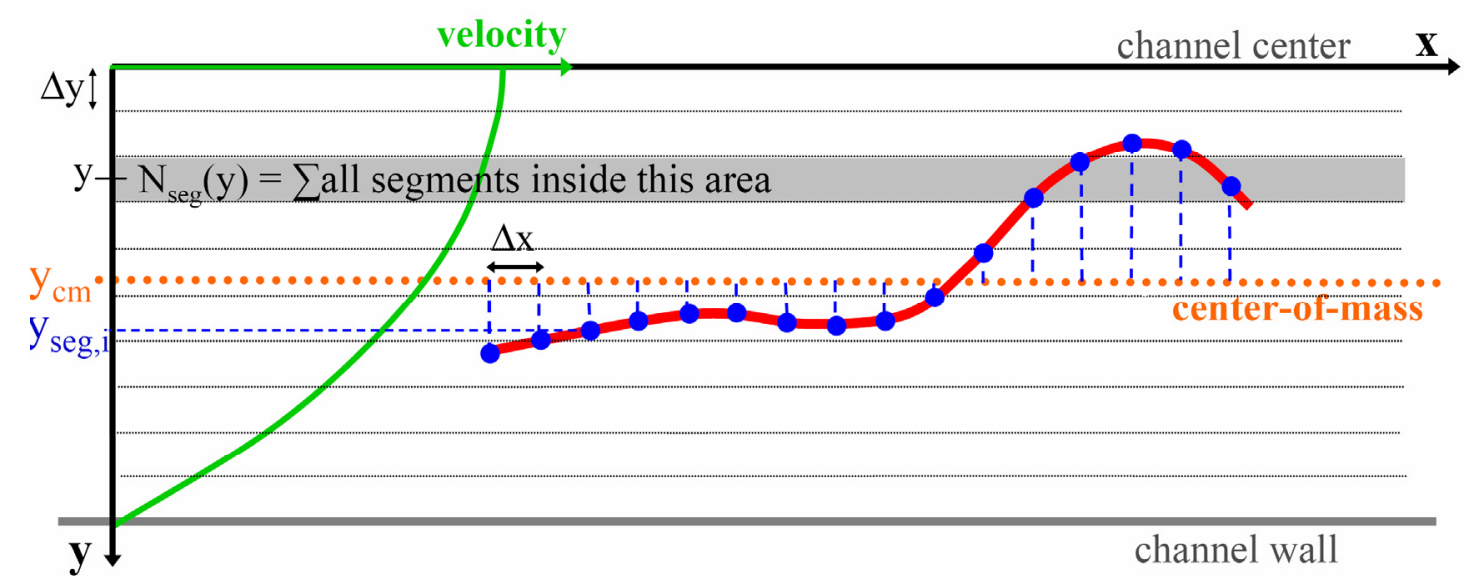

Figure 5-20: Analysis of the thermal fluctuations.

most filaments have an elongated shape $(>70 \%)$, we focus the investigation on these filaments and neglect the bent filaments. The position $y_{\text {seg }}$ of a segment in the $y$ direction is measured for each segment of a filament. Considering only filaments which have their center-of-mass at the same certain channel position $y_{c m}$, the numbers $N_{\text {seg }}$ of segments of these filaments inside intervals of $\Delta y=0.1 \mu \mathrm{m}$ along the $y$-axis are counted. The obtained segment distribution for the certain channel position $y_{c m}$ is normalized by the total number of segments. For each center-of-mass position, about 200 filaments are analyzed. Figure 5-21a shows the segment distributions for three center-of-mass positions $y_{c m}$ at a velocity $v_{0}=2.4 \pm 0.7 \mathrm{~mm} / \mathrm{s}$, and figure $5-21 \mathrm{~b}$ shows the segment distributions for filaments with the center-of-mass at $y_{c m}=2 \pm 0.5 \mu \mathrm{m}$ for different flow velocities.

All segment distributions have a maximum at the center-of-mass position of the filaments. The heights of the maximum are smaller and the distributions are wider for filaments closer to the channel center (figure 5-21a). Additionally, an increase of the flow velocities narrows the distributions and leads to larger maxima (figure 5-21b). Consequently, the deviations of segments from the center-of-mass streamline are, on average, larger for filaments near the channel center than at the walls and for filaments at smaller flow velocities. This is a consequence of the increase in the local shear rate toward the walls and for larger flow velocities. Larger shear rates elongate the filaments more strongly in the flow direction and therefore the extensions of the filaments perpendicular to flow direction are decreased, leading to the smaller deviations. As seen in figure 5-21, the distributions can approximately be described by Gaussian distributions with a maximum at $y_{\text {seg }}=y_{c m}$, although the tails of the distributions deviate slightly from the Gaussian distributions (see semi-logarithmic plots in figure 5-21c-d). 

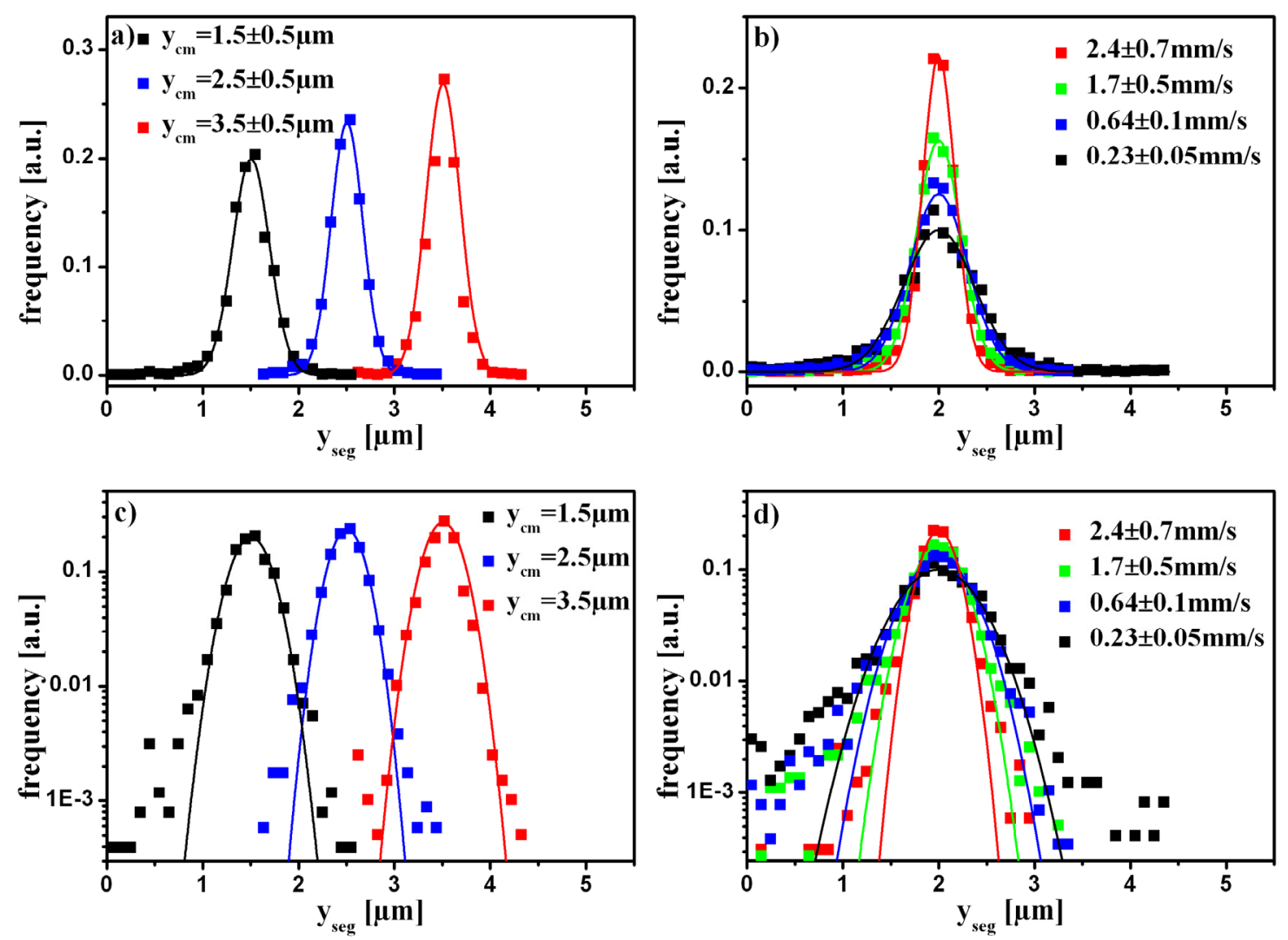

Figure 5-21: Segment distributions. a) Segment distributions at different channel positions for $v_{0}=2.4 \pm 0.7 \mathrm{~mm} / \mathrm{s}$. b) Segment distributions for filaments with center-of-mass at $y_{c m}=(2 \pm 0.5) \mu \mathrm{m}$ for different flow velocities. c) Semi-logarithmic plot of a). d) Semi-logarithmic plot of b). The channel center is at $y=0$ and the walls at $y=5.5 \mu \mathrm{m}$.

In the semi-logarithmic plots, it can additionally be seen that the distributions are slightly asymmetric. The segments deviations from the center-of-mass streamline are larger for segments found in direction toward the centerline $\left(y_{s e g}<y_{c m}\right)$ than in direction toward the walls $\left(y_{\text {seg }}>y_{c m}\right)$. This is a consequence of the large extensions of the filaments perpendicular to the flow direction. The local shear rate $\dot{\gamma}\left(y_{\text {seg }}\right)$ at the segments positions deviate from the shear rate $\dot{\gamma}\left(y_{c m}\right)$ at the center-of-mass position. For segments with $y>y_{c m}$, the local shear rate at its own positions is larger than for segments with $y<y_{\mathrm{cm}}$. Consequently, the contour of a single filament is unequally elongated and parts of the contour with $y>y_{c m}$ are more elongated ( $\hat{=}$ smaller deviation) than parts with $y<y_{c m}$. Thus, the conformational changes due to the position-dependent shear rate are not only seen in the segment distributions by comparing filaments at different positions, but also in the contour of a single filament. However, the differences in the deviations from the center-of-mass streamline for segments $y>y_{c m}$ and $y<y_{c m}$ are very small. Therefore, the position-dependent behavior of segments relative to the center-of-mass is neglected in the following discussions. 

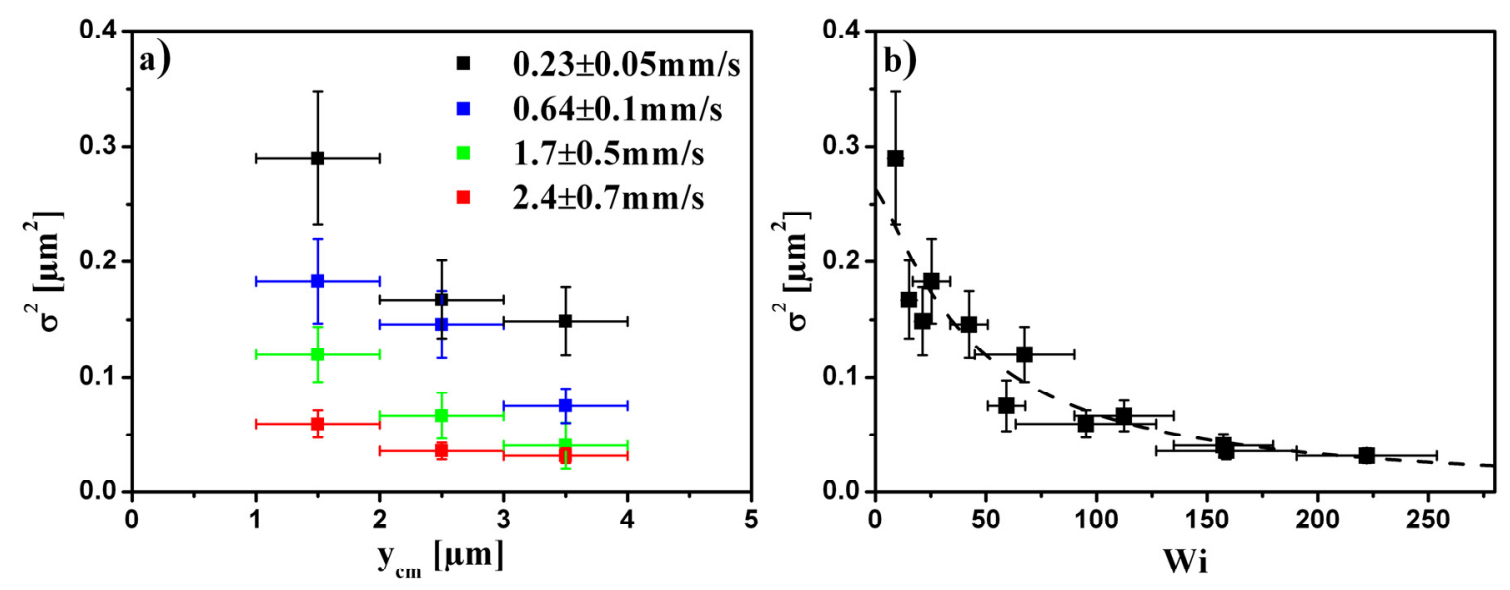

Figure 5-22: a) $\sigma^{2}$ against channel position for different velocities. b) $\sigma^{2}$ against Weissenberg number.

For filaments at a certain channel position $y_{c m}$, the mean deviation of the segments from the center-of-mass streamline can be quantified by the standard deviation

$$
\sigma=\sqrt{\sum_{i} \frac{1}{n_{s}}\left(y_{\text {seg }, i}-y_{c m}\right)^{2}},
$$

where $y_{\text {seg, } i}$ denotes the position measurement of a single segment and $n_{s}$ is the total number of segments. The mean square deviation $\sigma^{2}$ is plotted for all measured velocities against the channels positions in figure 5-22a. In this plot, the decrease of $\sigma^{2}$ toward the walls and at higher flow velocities is clearly seen.

Calculating the Weissenberg number (equation 4.17) by using the relaxation time $\tau_{\text {rel,exp }}=0.4 \mathrm{~s}$ and the shear rate $\dot{\gamma}(y)=8 v_{0} y_{c m} / d^{2}$, the dependence of $\sigma^{2}$ on the Weissenberg number is obtained and shown in figure $5-22 b$. The data points for the different velocities fall all at a single curve. Thus, the Weissenberg number $W i=\dot{\gamma} \tau_{\text {rel }}$ determines how large $\sigma^{2}$ is. A parameter $\widetilde{D}=\sigma^{2} / \tau_{\text {rel }}$ for a certain shear rate $\dot{\gamma}$ can be calculated which has the units of a diffusivity. The time $\tau_{\text {rel }}$ is the hydrodynamic time scale of the polymer system and characterizes the dynamics of the thermal fluctuations. The length $\sigma$, obtained by averaging the segment deviations from the center-of-mass streamline over many segments of several filaments, can also be considered as the typical distance a segment can reach in the time $\tau_{\text {rel }}$ due to thermal fluctuations (see figure 5-23). $\widetilde{D}$ decreases strongly with increasing shear rate as its is seen in figure 5-24 and presumably reflects the shear-dependent diffusion behavior of the actin filaments inside the microchannels. Therefore, it is reasonable to assume that the 
diffusivity $D$ of the actin filaments is proportional to the parameter $\widetilde{D}$,

$$
D(\dot{\gamma}) \propto \widetilde{D}(\dot{\gamma}),
$$

and therefore decreases with increasing shear rate. Consequently, the diffusivity of the actin filaments is position-dependent and decreases toward the walls. A decrease of the diffusivity toward the walls suggests cross-streamline migration in direction toward the walls which is consistent with the center-of-mass probability distributions.

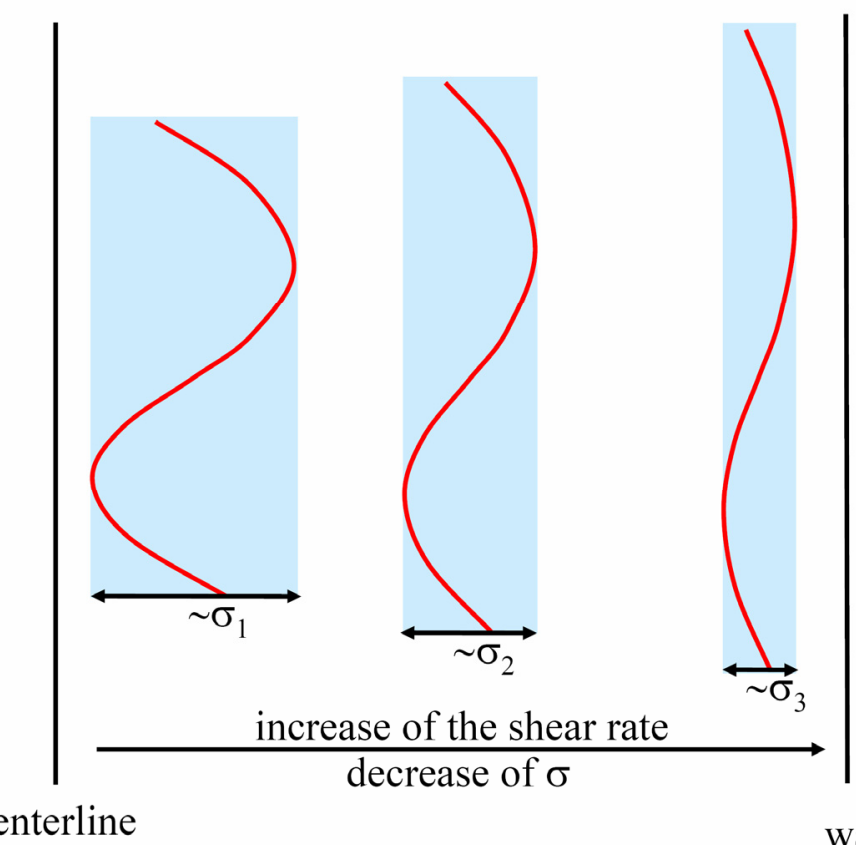

wall

Figure 5-23: Illustration of the "segment-diffusivity" of actin filaments inside microchannels.

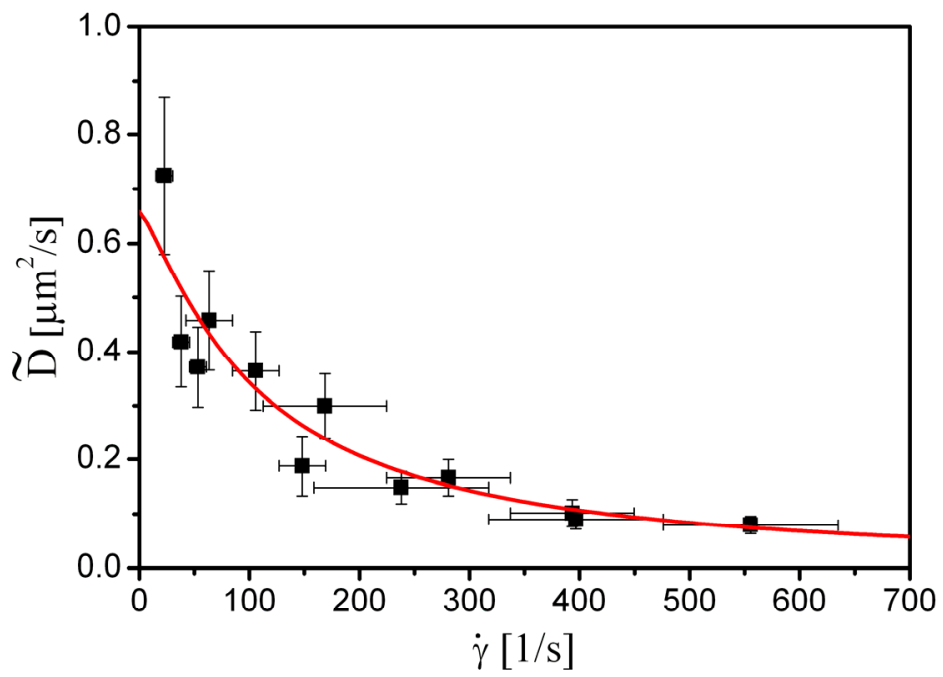

Figure 5-24: $\widetilde{D}$ against the shear rate. The line has the function $0.67 \mu \mathrm{m}^{2} / \mathrm{s} /\left(1+\left(9.8 \cdot 10^{-3} \mathrm{~s} \cdot \dot{\gamma}\right)^{1.25}\right)$. 
To obtain an expression for the spatially-varying diffusivity, $\widetilde{D}$ can be fitted with a function $\widetilde{D}=a /\left(1+(b \dot{\gamma})^{c}\right)$, where $a, b$ and $c$ are fit parameters. Using the relation between the channel position and the shear rate (equation 5.2), the position dependent diffusivity of the actin filaments can be written as

$$
D\left(y_{c m}\right)=\frac{D_{0} \cdot a}{\left(1+\left(b \frac{8 v_{0}}{d^{2}} y_{c m}\right)^{c}\right)},
$$

where $D_{0}$ is a proportional constant, and $a=0.67 \mu \mathrm{m}^{2} / \mathrm{s}, b=9.8 \cdot 10^{-3} \mathrm{~s}$, and $c=1.25$ are the results obtained from the fit.

After quantifying the spatially-varying diffusivity of the semiflexible actin filaments, the center-of-mass probability distribution $n\left(y_{c m}\right)$ can be determined by solving equation 5.23. Considering only one channel half and assuming that a filament is only influenced by the hydrodynamic interactions with a single wall, the governing equation of the center-of-mass probability distribution in steady-state can be simplified to

$$
-\frac{\widetilde{K}\left(y_{c m}\right)}{\left(d / 2-y_{c m}\right)^{2}} n-\frac{\partial D\left(y_{c m}\right)}{\partial y_{c m}} n-D\left(y_{c m}\right) \frac{\partial n}{\partial y_{c m}}=0,
$$

where $\widetilde{K}\left(y_{c m}\right)$ is given in equation 5.27 and $D\left(y_{c m}\right)$ in equation 5.30. This differential equation can be solved by

$$
n\left(y_{c m}\right)=\frac{n_{0}}{a}\left(1+\left(b \frac{8 v_{0}}{d^{2}} y_{c m}\right)^{c}\right) \exp \left(-\frac{K_{0}}{D_{0}} g\left(y_{c m}\right)\right)
$$

where

$$
g\left(y_{c m}\right)=\int\left(\frac{1}{a\left(d / 2-y_{c m}\right)^{2}}\left(\frac{8 v_{0}}{d^{2}} y_{c m}\right)^{2 / 3}\left(1+\left(b \frac{8 v_{0}}{d^{2}} y_{c m}\right)^{c}\right)\right) \mathrm{d} y_{c m},
$$

$a=0.67 \mathrm{~mm}^{2} / \mathrm{s}, b=9.8 \cdot 10^{-3} \mathrm{~s}$, and $c=1.25$. The integral can be calculated (appendix 8.1 ) with Mathematica (Version 6.0, Wolfram Research, Oxfordshire, UK). The parameter $n_{0}$ can be considered as normalization constant and $K_{0} / D_{0}$ can be interpreted as a weighting of the drift term due to hydrodynamic interactions with the wall and the drift term due to spatially-varying diffusivity.

For one parameter set [133], $n\left(y_{c m}\right)$ is shown in figure 5-25. In this parameter set, the channel width $d=11 \mu \mathrm{m}$ and the different velocities $v_{0}$ are chosen equal to the experiment. $K_{0} / D_{0}$ is kept constant for all velocities and only the constant $n_{0}$ varies for the different velocities. For this parameter set, $n\left(y_{c m}\right)$ shows for all velocities a minimum at the channel center and a maximum at a channel position somewhere between the 


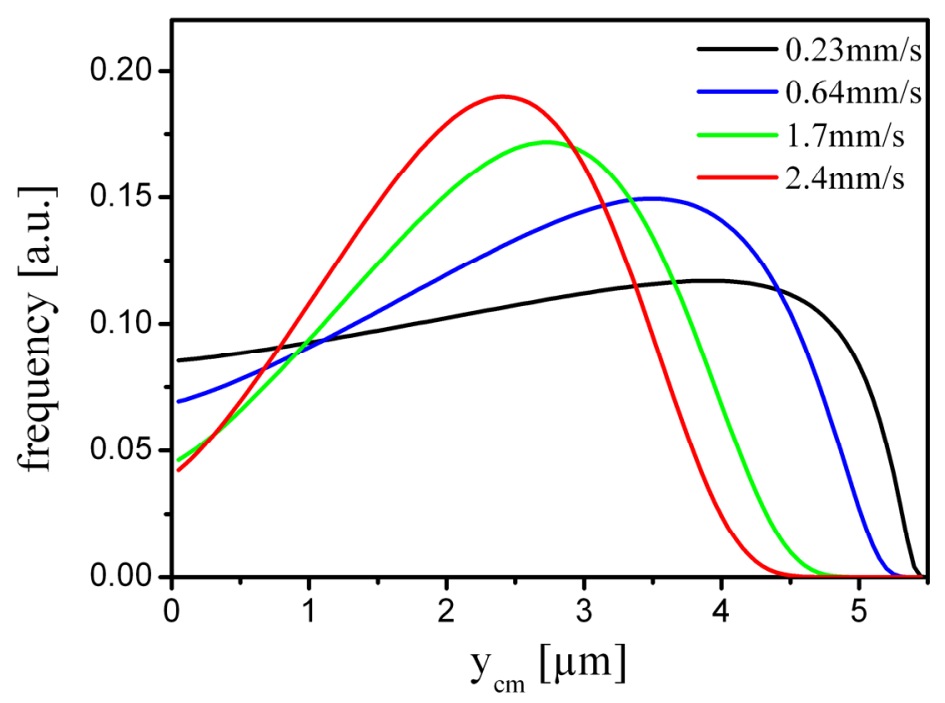

Figure 5-25: Center-of-mass probability distributions calculated for steady-state conditions for a parameter set [133]. In the governing equation of the center-of-mass probability distribution, hydrodynamic interactions with the wall and a spatially-varying diffusivity are included. The diffusivity is assumed to be proportional to the measured mean square deviation of segments from the center-of-mass streamline.

channel center and the channel walls. Whereas the minimum at the smallest velocity $v_{0}=0.23 \mathrm{~mm} / \mathrm{s}$ is slightly pronounced and the distribution can roughly be considered as constant, the minima become more pronounced and the peak heights of the maxima become larger with increasing velocities. Furthermore, the distributions decrease toward the wall. Depletion layers near the wall can be observed for which the thicknesses are larger at higher velocities. Thus, $n\left(y_{c m}\right)$ qualitatively shows the main features observed for the center-of-mass probability distributions of the semiflexible actin filaments (figure 5-17): almost equally distributed filaments for small velocities, a minimum at the channel center and a maximum between the channel center and the walls for large velocities, and depletion layers at the walls. The peak heights of the maxima are comparable to those of the measured distributions. For the two largest velocities, $n\left(y_{c m}\right)$ and the measured distributions are in agreement almost over the entire channel as seen in figure 5-26. The model is most suitable for large velocities for which steric interactions are negligible. Steric interactions, which are neglected in this model, generate a depletion layer already without flow and become smaller with increasing velocities. This leads to the much larger depletion layers in the experiment for small velocities compared to the calculations. 


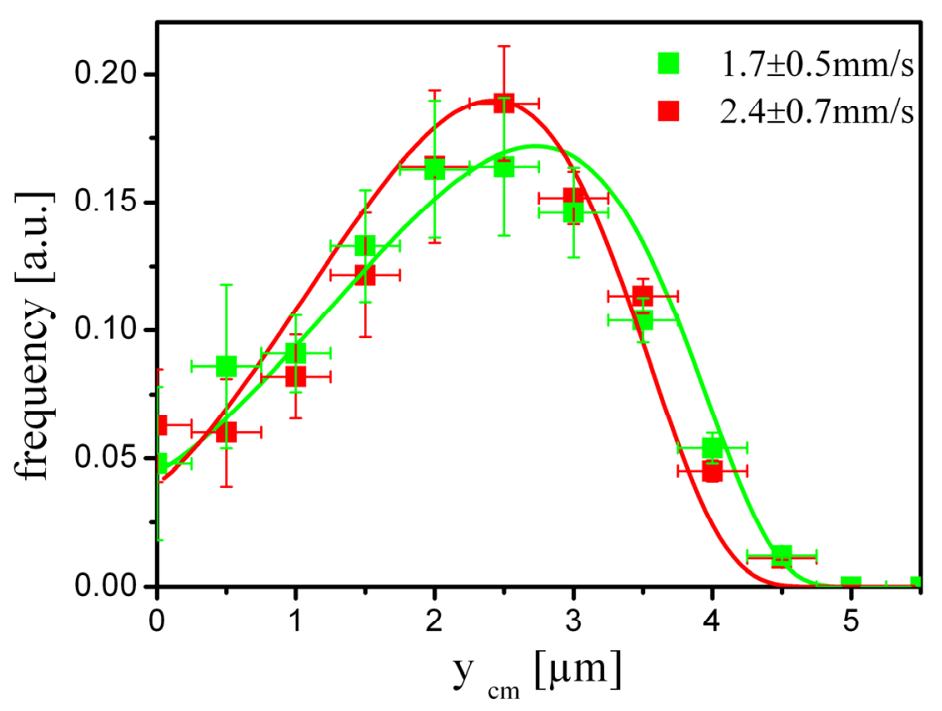

Figure 5-26: Comparison of the calculated center-of-mass probability distribution $n\left(y_{\mathrm{cm}}\right)$ with the measurements for actin filaments at large velocities.

In summary, we measured the center-of-mass probability distributions of actin filaments in flow along a cross-section of a straight channel for different velocities. For small velocities, the filaments are almost homogenously distributed over the channel. In contrast, a striking local minimum is found at the channel center for large velocities. This minimum becomes more pronounced with increasing velocities. Additionally, depletion layers near the walls are observed. These results can be explained by kinetic arguments. Spatially-varying diffusivity causes cross-streamline migration away from the channel center, resulting in a local minimum at the channel center. Hydrodynamic interactions with walls lead to cross-streamline migration away from the channel walls, creating depletion layers near the walls. For small velocities, steric interactions influence additionally the depletion layers. The dependence of the diffusivity on the shear rate is measured by assuming proportionality between the diffusivity and the mean square deviation of segments from the center-of-mass streamline. Including this diffusivity and hydrodynamic interactions with the wall into the center-of-mass flux, the center-of-mass probability distribution is calculated from the condition that the centerof-mass flux has to be zero in steady-state. For suitable weighting of the two drift terms, the calculated center-of-mass probability distributions for the different velocities show the same characteristics as observed in the measured distributions. For large velocities, an approximately quantitative agreement is even obtained. In particular, the striking minima at the channel center are well reproduced for which the shear rate dependence in the measured diffusivity is responsible. 


\subsection{Curved Channels}

In the previous sections of this chapter, the behavior of actin filaments in pressuredriven flow is analyzed. The conformations and orientations of the semiflexible filaments are discussed and in particular the center-of-mass probability distributions for different flow velocities are considered. For all these investigations, experiments are performed using a simple rectilinear geometry with an aspect ratio (depth/width) of unity. However, the results discussed for the straight channels are not necessarily valid for other geometries. The knowledge of the behavior inside different geometries might be used to manipulate the polymer in a controlled manner. In order to understand the influence of the channel geometry, the behavior of actin filaments inside curved channels with a constant curvature (see figure 5-27a) is considered in this section. The channels, with a width of $d=11 \pm 1 \mu \mathrm{m}$ and depth of $10 \pm 1 \mu \mathrm{m}$, have the same dimensions as in the experiments in straight channels. The optical focal plane of the microscope is again adjusted to the half the channel height. The radius of curvature of the channels is $R_{c}=15 \pm 1 \mu \mathrm{m}$.
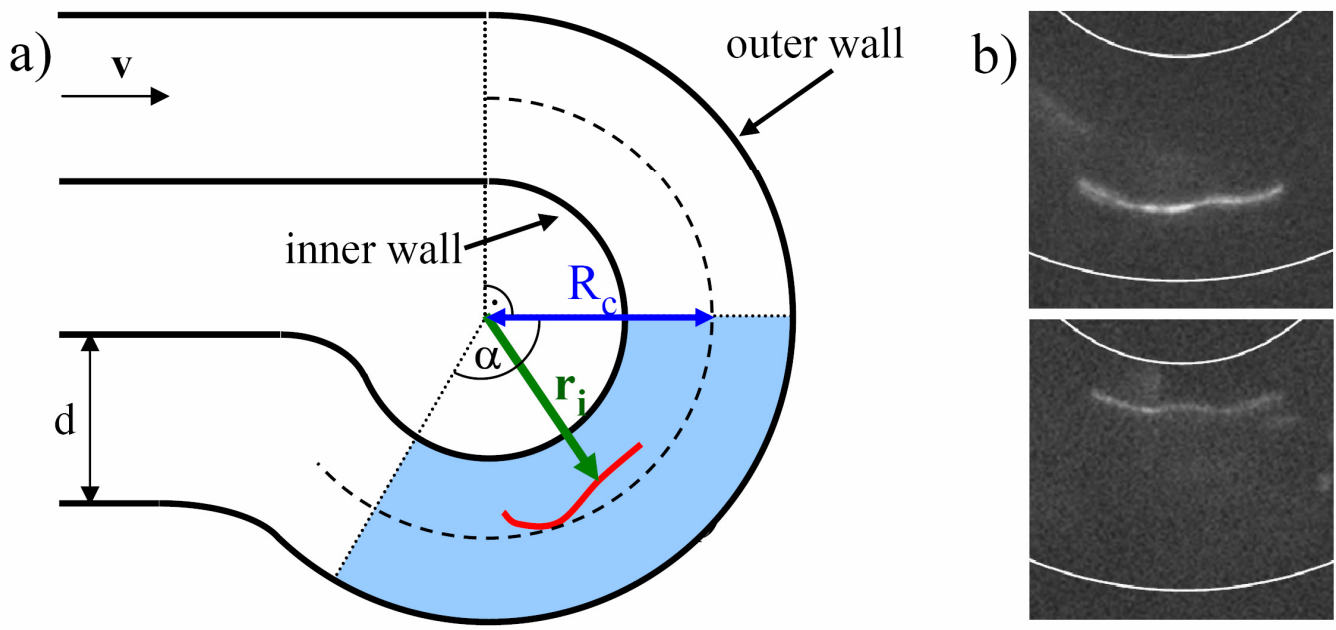

Figure 5-27: Actin filaments inside curved channels. a) Curved channel geometry. $d$ : width of the channel, $R_{c}$ : radius of curvature, $\mathrm{r}_{\mathrm{i}}:$ position of the $i$-th pixel of a filament. Filaments are analyzed inside the blue area $\left(\alpha=120^{\circ}\right)$. b) Snapshots of flowing actin filaments inside curved channels $\left(v_{0} \approx 1 \mathrm{~mm} / \mathrm{s}, d \approx 11 \mu \mathrm{m}, R_{c} \approx 15 \mu \mathrm{m}\right)$.

Typical snapshots of actin filaments inside curved channels are shown in figure 5-27b. The actin filaments adapt to the geometry and therefore they are slightly bent. Additionally, tumbling filaments and parabolic bent filaments are also observed in analogy to the straight channels (see section 5.1). 
According to the geometry, positions are measured in polar coordinates and the centerof-mass is characterized by the radius

$$
r_{c m}=\frac{1}{n_{p}} \sum_{i=1}^{n_{p}} r_{i}-R_{c},
$$

where $r_{i}$ is the radius of each pixel along the pixelline and $n_{p}$ is the total number of pixels. This corresponds to the center-of-mass position $y_{c m}$ along a cross-section of the channel with channel center at $y_{c m}=0$ and walls at $y_{c m}= \pm d / 2$. All actin filaments found at the end of the curved channel (see blue area in figure 5-27a) are analyzed.
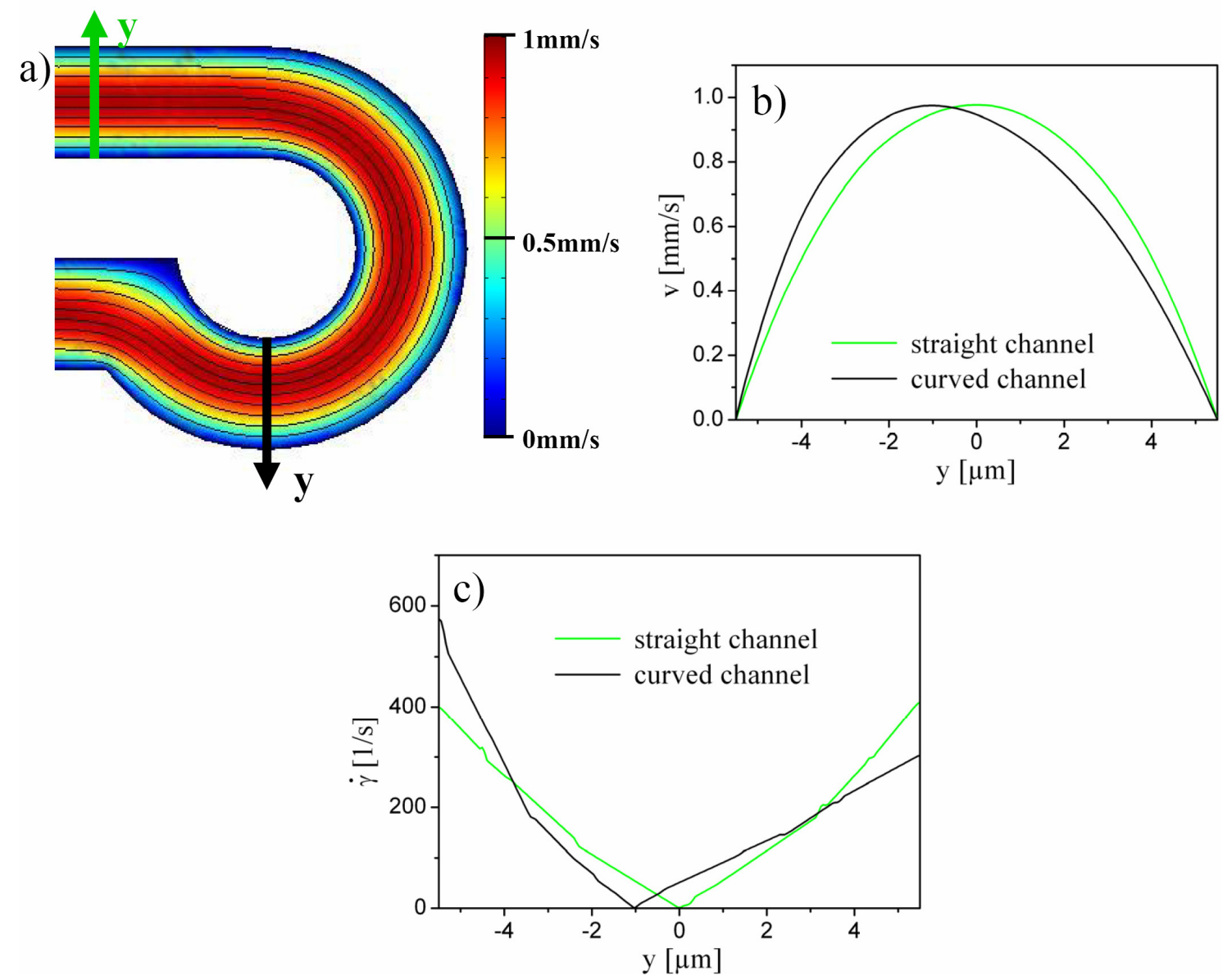

Figure 5-28: a) FEM-simulation of the velocity field (the picture shows a slice of a 3D-simulation at the focal plane). Black lines are streamlines of the velocity field. b) Velocity profiles along the crosssection in a straight channel and in the curved channel $(y=0$ : centerline, $y=5.5 \mu \mathrm{m}$ : outer wall, $y=-5.5 \mu \mathrm{m}$ : inner wall). c) Shear rate versus position $y$.

A FEM simulation of the flow field inside the curved channel is shown in figure 5-28a. The velocity profile along a cross-section of the channel, seen in figure $5-28 \mathrm{~b}$, is asymmetric and the maximum of the velocity is shifted toward the inner wall. This is a consequence of the different lengths of the inner and outer walls with no-slip boundary 
conditions. Solvent molecules in the channel half at the longer outer wall experience on average a larger internal friction from the neighboring solvent molecules than molecules in the channel half at the inner wall. Therefore, the molecules at the longer outer wall flow more slowly.
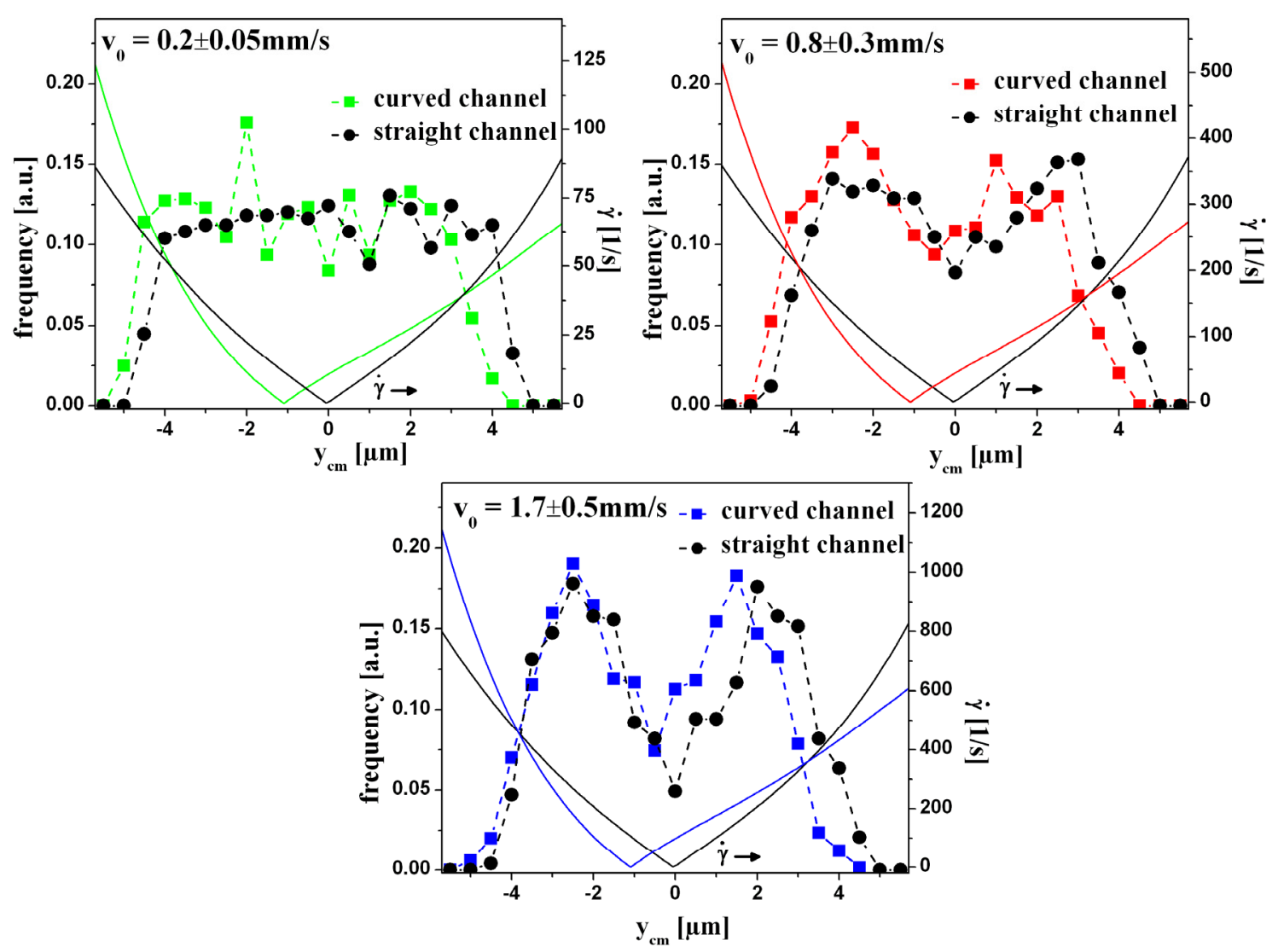

Figure 5-29: Center-of-mass probability distributions in curved channels for different velocities. For comparison, the distributions inside the straight channels are plotted. Additionally, the shear

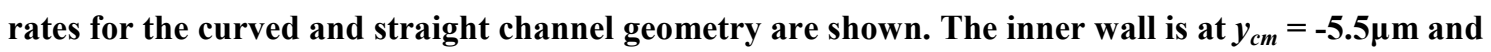
the outer wall at $y_{c m}=5.5 \mu \mathrm{m}$.

The center-of-mass probability distributions of actin filaments inside curved channels are shown for three velocities in figure 5-29. The distributions are normalized for each center-of-mass position by the circular arc length of the analyzed area and by the number of analyzed filaments. For comparison, the center-of-mass probability distributions for straight channels of comparable velocities are also plotted. For a small velocity $v_{0} \approx 0.2 \mathrm{~mm} / \mathrm{s}$, the distribution is constant almost over the entire channel. Only small depletion layers at both walls can be seen, for which the thickness is larger at the outer wall than at the inner wall. In contrast, the distributions for larger velocities have local minima which become more pronounced with larger flow velocities. Additionally, 
the depletion layer thicknesses are increased. Thus, the basic characteristics of the center-of-mass probability distributions in curved channels are the almost same as in straight channels and can also be explained with the same arguments: migration away from the channel center due to a spatially-varying diffusivity and migration away from the walls due to hydrodynamic interactions. However, the center-of-mass probability distributions are not symmetric relative to the channel center such as it is observed in the straight channels. The minima of the distributions are not directly found at the channel center and the thicknesses of the depletion layers at the inner walls are clearly smaller than at the outer walls. It seems that the entire distributions are shifted toward the inner walls.

As discussed in the last section, cross-streamline migration due to a spatially-varying diffusivity strongly influences the center-of-mass probability distributions. In order to obtain information about the migration behavior in the curved geometry, the thermal fluctuations of the filaments are analyzed by measuring the segment distributions. Furthermore, the center-of-mass probability distributions are calculated for steady-state conditions.
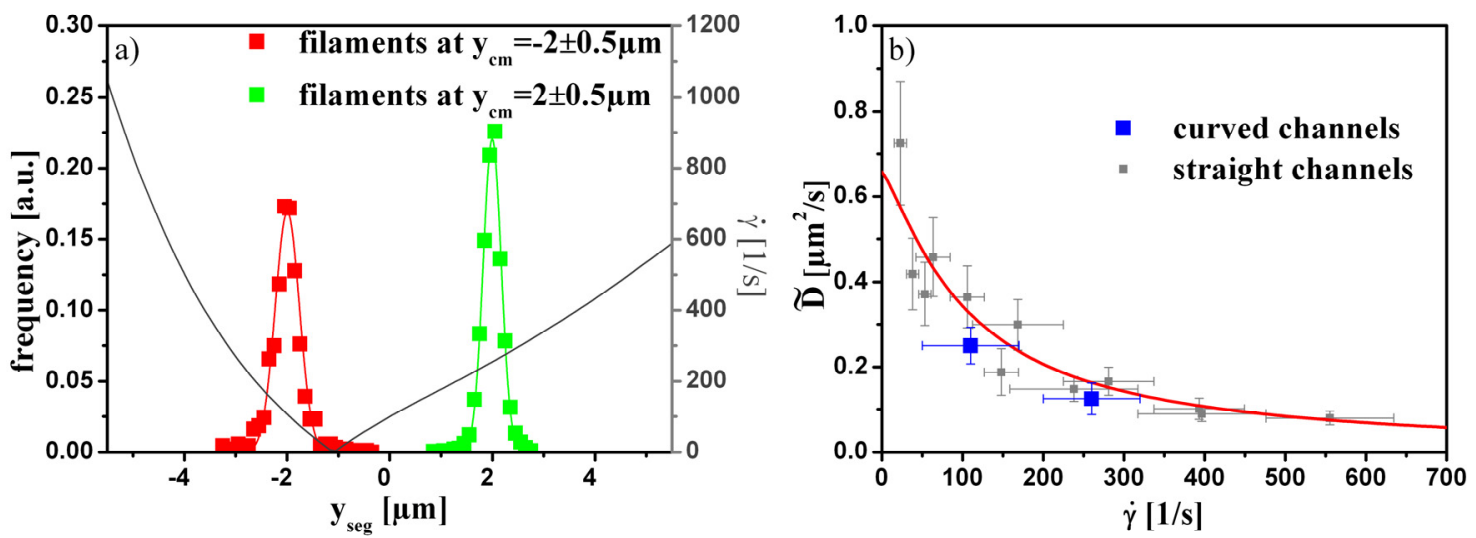

Figure 5-30: a) Segment distributions inside curved channel for $v_{0}=1.7 \pm 0.5 \mathrm{~mm} / \mathrm{s}$ at two channel positions. The lines are Gaussian distributions. The inner wall is at $y_{c m}=\mathbf{- 5 . 5} \mu \mathrm{m}$ and the outer wall is at $y_{c m}=5.5 \mu \mathrm{m}$. b) $\widetilde{D}$ against the shear rate.

Analogous to the straight channels, the segment positions $y_{\text {seg }}$ are measured and the frequencies of segments at certain channel positions are counted for all filaments with center-of-mass position $y_{\mathrm{cm}}$. The segment distributions are normalized by the total number of analyzed segments. Two segment distributions are shown in figure 5-30a for a velocity $v_{0} \approx 1.7 \mathrm{~mm} / \mathrm{s}$. The two chosen positions, $y_{c m} \approx-2 \mu \mathrm{m}$ and $y_{c m} \approx 2 \mu \mathrm{m}$, have the same distances from the channel center. The distribution is narrower and the maximum is larger for filaments at $y_{c m} \approx 2 \mu \mathrm{m}$ (outer channel half) than at $y_{c m} \approx-2 \mu \mathrm{m}$ (inner 
channel half). Consequently, the mean square deviation $\sigma^{2}$ of segments from the center-of-mass streamline is smaller at $y_{c m} \approx 2 \mu \mathrm{m}$ than at $y_{c m} \approx-2 \mu \mathrm{m}$. The geometrical influence on the thermal fluctuations due to the channel curvature is small [134]. Thus, the results can be related to the larger shear rate $\dot{\gamma}\left(y_{c m}=2 \mu \mathrm{m}\right) \approx 260 \mathrm{~s}^{-1}$ compared to $\dot{\gamma}\left(y_{c m}=-2 \mu \mathrm{m}\right) \approx 110 \mathrm{~s}^{-1}$. Calculating $\widetilde{D}=\sigma^{2} / \tau_{r e l}$ for the two positions, the data points are in agreement with the measurements in the straight channels as seen in figure 5-30b. Therefore, it is reasonable to assume the same shear rate dependence for $\widetilde{D}$ as in the straight channels. The center-of-mass flux in the curved geometry in $y$-direction is given by

$$
j=-\frac{\widetilde{K}\left(y_{c m}\right)}{\left(d / 2-y_{c m}\right)^{2}} n_{c}+\frac{\widetilde{K}\left(y_{c m}\right)}{\left(-d / 2-y_{c m}\right)^{2}} n_{c}-\frac{\partial D\left(y_{c m}\right)}{\partial y_{c m}} n_{c}-D\left(y_{c m}\right) \frac{\partial n_{c}}{\partial y_{c m}},
$$

where $n_{c}\left(y_{c m}\right)$ is the center-of-mass probability distribution for the curved geometry, $D=D_{0} \cdot \widetilde{D}\left(\dot{\gamma}\left(y_{c m}\right)\right)$ and $\widetilde{K}\left(y_{c m}\right)=K_{0} \cdot \dot{\gamma}\left(y_{c m}\right)^{2 / 3}$. For each wall, the drift due to hydrodynamic interactions with this wall is approximated by the expression (see equation 5.25) for a single wall. An additional drift of polymers predicted due to misalignment of the relaxing force of the elongated polymers and the curved flow [23] is not included. For steady-state conditions, the center-of-mass flux has to be zero. The shear rate $\dot{\gamma}\left(y_{c m}\right)$ along the cross-section is obtained by FEM-simulations. The equation can be solved numerically with Mathematica and $n_{c}\left(y_{c m}\right)$ is shown for different velocities in figure 5-31. For the constant $K_{0} / D_{0}$, the same value [133] is taken as for the straight channels.

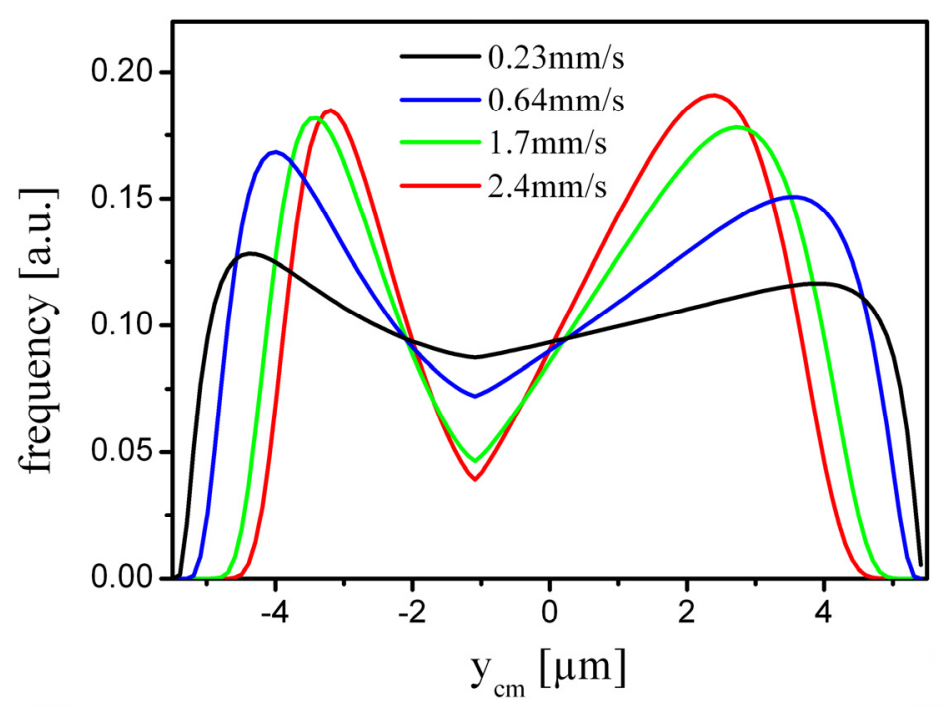

Figure 5-31: Calculated center-of-mass probability distributions $n_{c}\left(y_{c m}\right)$ for the curved geometry in steady-state. The channel center is at $y_{c m}=0$, the inner wall at $y_{c m}=-5.5 \mu \mathrm{m}$ and the outer wall at $y_{c m}=5.5 \mu \mathrm{m}$. 
The calculated center-of-mass probability distributions $n_{c}\left(y_{c m}\right)$ have for each velocity a local minimum, a maximum in each channel half, and depletion layers at the walls. The minima and the maxima become more pronounced and the depletion layer thicknesses increase with increasing velocities. These results are comparable to the straight channels. But other than in the straight channels, $n_{c}\left(y_{c m}\right)$ is asymmetric. The minimum is not at the centerline, but rather at $y_{c m}=-1 \mu \mathrm{m}$. Thus, the minimum is shifted to the channel position at which the shear rate is zero. Furthermore, the maximum is closer to the wall in the inner channel half compared to the outer channel half. The peak heights of the two maxima are only slightly different and also the depletion layers at the walls have comparable sizes for each velocity. The kink of $n_{c}\left(y_{c m}\right)$ at the minima is unphysical and is only caused by the assumed diffusivity, which has a discontinuity at the channel center.

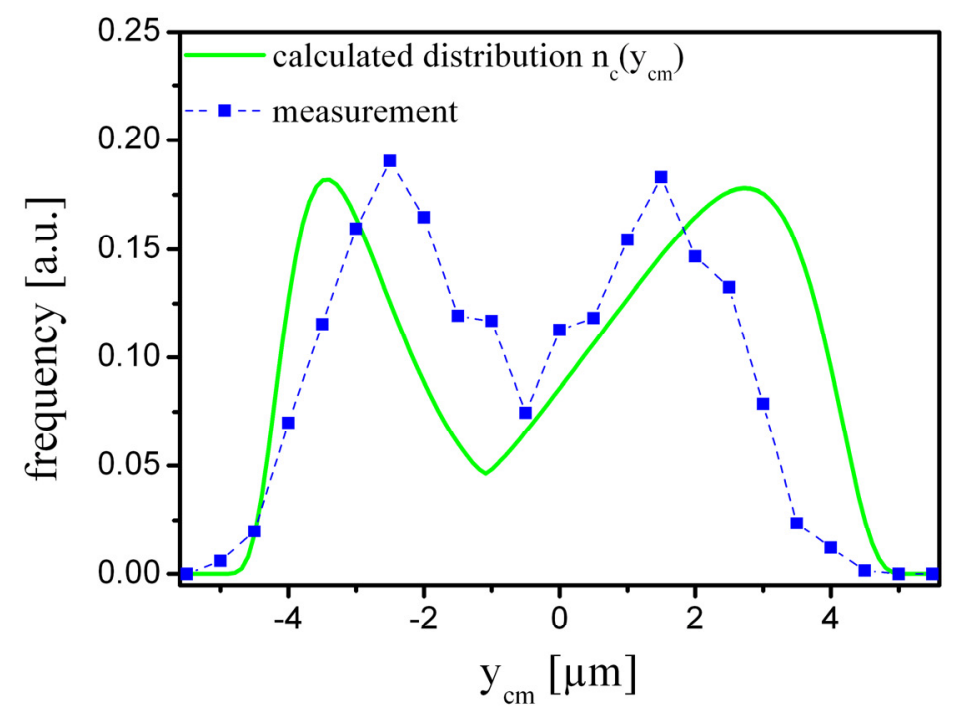

Figure 5-32 Comparison of the calculated center-of-mass probability distribution $n_{c}\left(\mathbf{y}_{c m}\right)$ for $v_{0}=1.7 \mathrm{~mm} / \mathrm{s}$ in steady-state with the measurement for actin filaments inside the curved channel geometry.

For comparison, figure 5-32 shows the calculated center-of-mass probability distribution $n_{c}\left(y_{c m}\right)$ and the measured distribution for a velocity $v_{0} \approx 1.7 \mathrm{~mm} / \mathrm{s}$ in the same plot. Both distributions are asymmetric and the minima are shifted toward the inner wall. However, the shift of the minimum is smaller for the measured data points. Furthermore, the depletion layers at both walls have comparable sizes for the calculated distribution, but are clearly different in the measurement. An important assumption, which we have made to calculate the center-of-mass probability distribution, is the steady-state condition. However, the actin filaments flow only a short time ( $\bar{t} \approx 30 \mathrm{~ms})$ inside the curved part of the microchannels, due to the design of the microfluidic device 
(see figure 5-27). The distances which they can migrate in this time are therefore probably small, and steady-state conditions cannot necessarily be expected.

The initial conditions in the experiment are the symmetric center-of-mass probability distributions of the straight channels before the filaments flow in the curved part of the channels. Inside the curved geometry, the filaments will be redistributed. Considering a certain streamline, the distance between this streamline and the inner wall decreases in the curved channel part as seen in the FEM-simulation in figure 5-28a. Consequently, the filaments are shifted toward the inner wall by assuming that the filaments primarily follow the streamlines. Thus, redistribution of filaments occurs in the curved part of the microchannel without the need of any migration or diffusion. Using the calculated center-of-mass probability distribution $n\left(y_{c m}\right)$ for the straight channels as initial conditions of the filaments distribution, the redistributions of filaments due to the streamline displacements generate the asymmetric center-of-mass distribution $n_{s}\left(y_{c m}\right)$ shown in figure 5-33. This distribution $n_{s}\left(y_{c m}\right)$ is calculated by shifting the channel positions by the streamline displacements obtained from the FEM-simulation. Comparison of $n_{s}\left(y_{c m}\right)$ with the measurement shows that the profiles of the distributions in the middle of the channel is very similar. $n_{s}\left(y_{c m}\right)$ fits better to the measured data than the calculated distribution $n_{c}\left(y_{c m}\right)$ for steady state conditions. In particular, the minimum

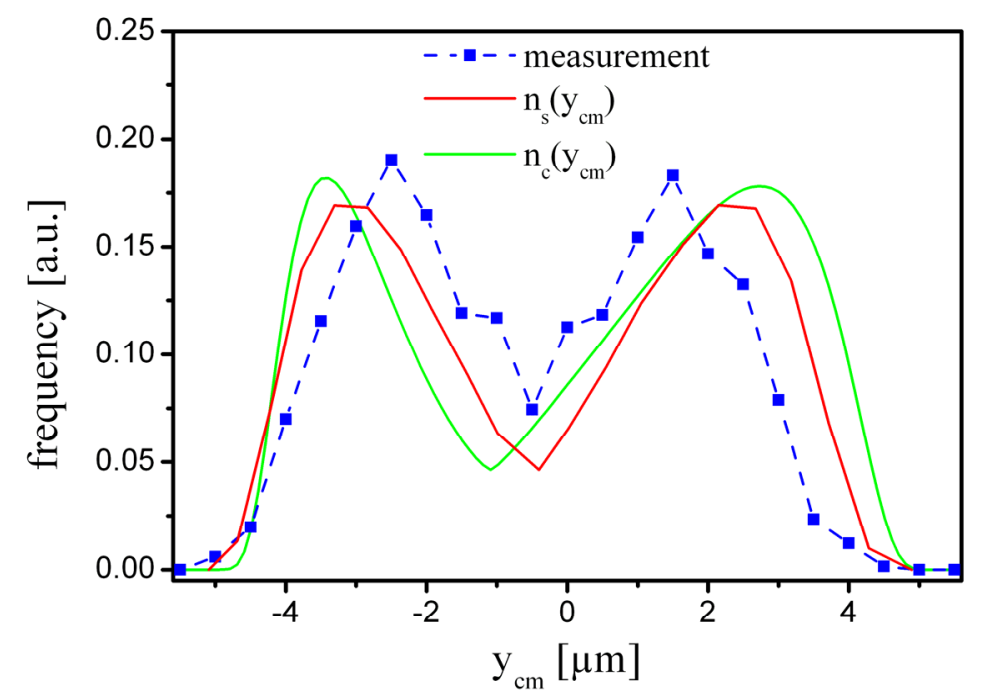

Figure 5-33: Redistribution of actin filaments due to streamline displacement. The plot shows an comparison of the measurement with the calculated center-of-mass probability distribution $n_{s}\left(y_{c m}\right)$ obtained by shifting the channel positions of the center-of-mass distribution $n\left(y_{c m}\right)$ for the straight channel according to the streamline displacements. Additionally, the distribution $n_{c}\left(y_{c m}\right)$ is plotted which was calculated for steady-state conditions. The velocity for all shown distributions is $v_{0} \approx 1.7 \mathrm{~mm} / \mathrm{s}$. 
is found at the same channel position. This indicates that for short times the streamline displacement is the main cause for the asymmetry in the center-of-mass probability distribution. For long times for which steady-state conditions can be assumed, the calculated distribution $n_{c}\left(y_{c m}\right)$ suggests that the asymmetry becomes even larger. The local minimum will be more shifted toward the inner wall to the channel position for which the local shear rate is zero. However, the depletion layer at the outer wall is clearly larger in the measurement than in both calculated distributions $n_{c}\left(y_{c m}\right)$ and $n_{s}\left(y_{c m}\right)$. Apparently, additional geometry effects influence the center-of-mass probability distribution. For curved geometries, a further cause of migration has been predicted which has not been included in any of our calculations, namely the misalignment of the relaxing force and the curved flow [23]. The direction of this migration is suggested toward the inner wall and this might explain the larger measured depletion layer at the outer wall.

In summary, we measured the center-of-mass probability distributions of actin filaments inside curved microchannels with constant curvature for different velocities. Analogous to the straight channels considered in the last section, the distributions have depletion layers near the walls and a local minimum for large velocities. In contrast to the straight channels, the distributions are asymmetric and the local minimum is shifted toward the inner wall. Additionally, the depletion layer at the outer wall is larger than at the inner wall. We showed for one velocity that this asymmetry is mainly caused by the displacement of streamlines in the curved channel part due to the design of the microfluidic device. In the experiment, the actin filaments are inside the curved channel part for only a short time in which steady-state is not reached. Calculations of the center-of-mass probability distribution for steady-state conditions suggest that the distribution becomes more asymmetric for later times and in particular the local minimum is more shifted toward the inner wall. 


\section{Chapter 6}

\section{Actin Bundles}

This chapter discusses the bundling of actin filaments in vitro. More specifically, the mechanical properties of actin bundles and the time evolution of the formation are considered. Experiments are performed, in which the bundling is induced by the actinbinding protein (ABP) $\alpha$-actinin or by counterion condensation in the presence of $\mathrm{Ca}^{2+}$. The fluorescently labeled actin filaments are observed by fluorescence microscopy and the intensity of the emitted light gives us a measure about the number of filaments inside a bundle. Analysis of the thermal shape fluctuations of bundles provides information about their mechanical behaviors. Furthermore, bundling kinetics are studied by using a hydrodynamic focusing device which allows in a time-resolved manner the observation of the bundling.

\subsection{Mechanisms of Actin Bundle Formation}

Actin filaments are organized into bundles inside cells (see chapter 1.2). Within a cell, ABPs are responsible for the bundle formation. Typically, these ABPs have two binding sites that interact with a specific subdomain of actin and thereby crosslink two actin filaments (see figure 6-1a). The structure and behavior of the actin bundles depend strongly on properties of the linker molecules. For the relatively long actin-binding protein $\alpha$-actinin (length about $40 \mathrm{~nm}$ ), the actin filaments are organized into a loosely packed, disordered square lattice [60].

Due to the electrostatic nature of actin, actin bundles are also formed in the presence of multivalent ions by counterion condensation (see figure 6-1b) [44, 45]. Actin filaments are overall negatively charged polyelectrolytes (charge density about $-4 \mathrm{e}^{-} / \mathrm{nm}$ at physiological conditions [135]) and they attract cations from the buffer solution. Longrange attractions, resulting from fluctuations in the counterion charge density along the 
polyelectrolytes, can become larger than the repulsion of the equally charged polyelectrolytes at high ion concentration $[58,61]$. The consequence is the formation of actin bundles consisting of close packed actin filaments. The cations between the actin filaments are correlated and organized into density waves that are coupled with an additional twist of the helical inhomogeneously-charged filaments to optimize the charge distribution inside the actin bundles [61].

a)

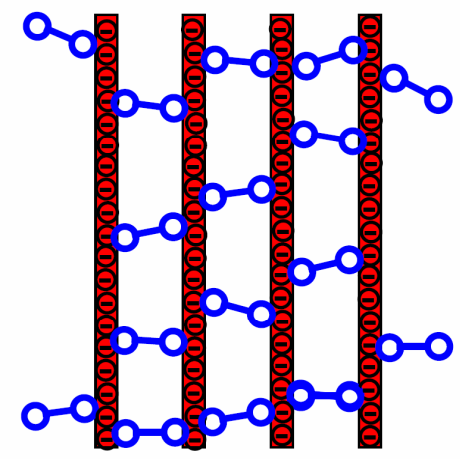

b)

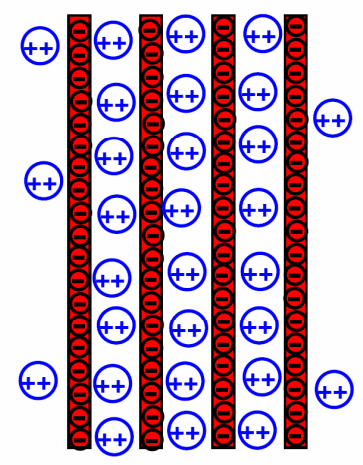

c)

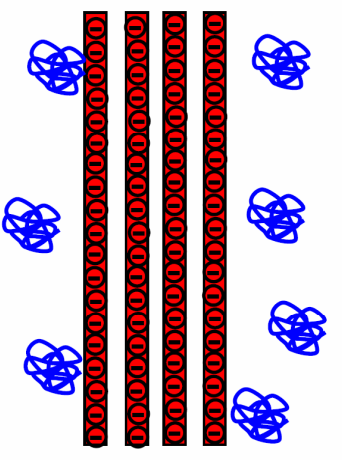

Figure 6-1: Actin bundle formed a) by ABPs (e.g. $\alpha$-actinin), b) by polyvalent counterion condensation, and c) by depletion forces.

Besides electrostatic interactions, unspecific binding between actin filaments leading to actin bundling can also be purely entropic [54, 55]. Actin filaments are bundled by depletion forces in the presence of coiled polymers such as poly(ethylene glycol) (PEG), (see figure 6-1c). The polymers are excluded from the actin filaments within a so-called depletion zone that has a thickness on the order of the radius of gyration of the polymers. When two filaments come close together, the two depletion zones around the filaments overlap. The gain in total free volume which the polymers can occupy leads to an increase in the configurational entropy. The consequence is an effective attraction between the actin filaments and formation of close packed actin bundles [54].

\subsection{Bending of Actin Bundles}

Two limited models that describe the bending of actin bundles are illustrated in figure 6-2 [67]. In the decoupled case (figure 6-2a), the actin filaments inside the bundle bend independently. A sliding of actin filaments against each other occurs without any resistance from the linker molecules, which are sheared or redistributed. In the fully coupled case (figure 6-2b), the actin filaments are hold close together by the linker 
a)

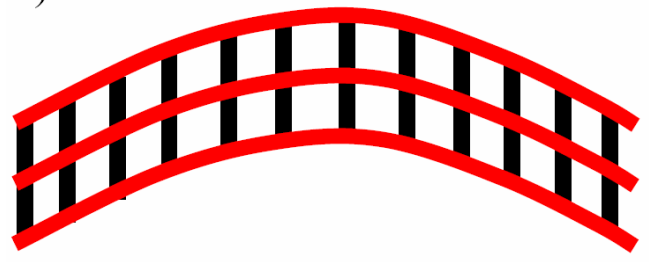

b)

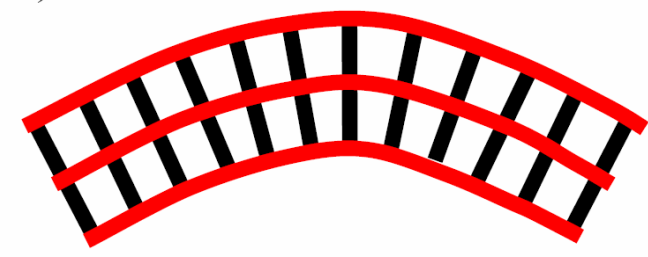

Figure 6-2: Actin bundle models: a) decoupled filaments and b) fully coupled filaments.

molecules, which resist an interfilament shearing of the bundle. Consequently, the filaments are forced to an additional stretching or compression. This costs energy. Therefore, bundles in the fully coupled case are stiffer than in the decoupled case.

In the decoupled case, the actin filaments inside the bundle all have the same bent shape. To bend the bundle, the same bending energy (see equation 3.2)

$$
\mathcal{H}_{\text {filament }}=\int_{\text {contour }} d s \frac{1}{2} \kappa_{\text {filament }}\left(\frac{\partial^{2} \mathbf{r}}{\partial s^{2}}\right)^{2},
$$

is needed to bend each filament, where $\kappa_{\text {filament }}$ is the bending rigidity of a single filament. The bending energy of the bundle with bending rigidity $\kappa_{\text {bundle, }}$

$$
\mathcal{H}_{\text {bundle }}=\int_{\text {contour }} d s \frac{1}{2} \kappa_{\text {bundle }}\left(\frac{\partial^{2} \mathbf{r}}{\partial s^{2}}\right)^{2},
$$

can also be calculated by the sum of the bending energy of all actin filaments

$$
\begin{aligned}
\mathcal{H}_{\text {bundle }} & =\sum_{i=1}^{n} \mathcal{H}_{\text {filament }}=n \mathcal{H}_{\text {filament }} \\
& =n \int_{\text {contour }} d s \frac{1}{2} \kappa_{\text {filament }}\left(\frac{\partial^{2} \mathbf{r}}{\partial s^{2}}\right)^{2},
\end{aligned}
$$

where $n$ is the number of filaments inside the bundle. Thus, the bending rigidity of the entire bundle

$$
\kappa_{\text {bundle }}=n \kappa_{\text {filament }},
$$

is proportional to the number of filaments inside the bundle.

Since the persistence length is proportional to the bending rigidity (see equation 3.1), the persistence length depends linearly on the number $n$ of filaments

$$
L_{p} \propto n
$$

in the decoupled case. 


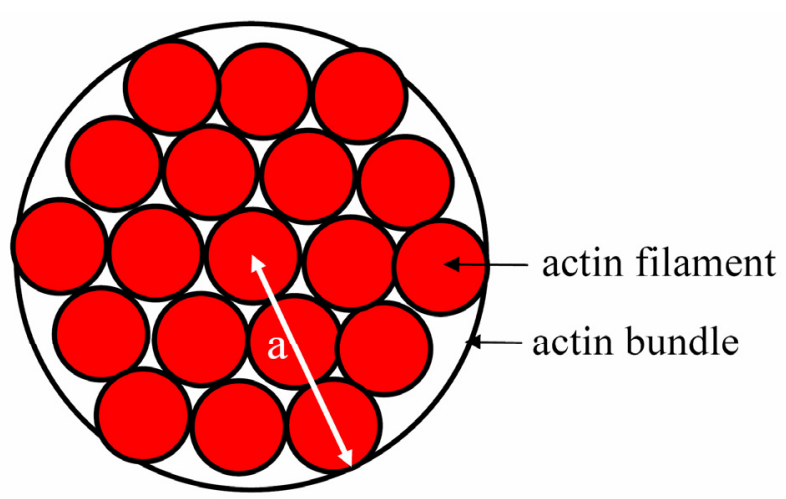

Figure 6-3: Cross-section of a bundle.

For the fully coupled case, the bending of the bundle can be considered as the bending of one single mechanical beam with an almost homogenous density and a circular crosssection (see figure 6-3). The bending rigidity of a mechanical beam is given by [81]

$$
\kappa=E I,
$$

where $E$ is the Young modulus and $I=\pi a^{4} / 4$ [81] is the second moment of inertia of a circular cross-section with radius $a$. Therefore, the persistence length, which is proportional to the bending rigidity (see equation 3.1 ), results in

$$
L_{p} \propto a^{4} .
$$

The cross-section area $A$ of a bundle is proportional to the number $n$ of filaments inside the bundle and is also proportional to the square of the radius for a circular cross-section:

$$
A \propto n \propto a^{2} .
$$

Using the relations in equation 6.7 and 6.8 , the dependence of the persistence length on the number of filament is given by

$$
L_{p} \propto n^{2}
$$

for the fully coupled case.

\subsection{Fluctuating Actin Bundles}

\subsubsection{Experiment and Analysis}

To form actin bundles by counterion condensation, an actin solution (70nM) is mixed carefully 3:7 with a $10 \mathrm{mM} \mathrm{CaCl}_{2}$ solution. The solution is equilibrated for $1-20 \mathrm{~min}$ to give the actin filaments time to form bundles with different thicknesses. A drop (1 $\mu 1)$ of the solution is placed at a microscope slide and a cover slip is put on top of the solution (see figure 6-4). Glue seals the microscope slide and the cover slip to avoid evaporation. 


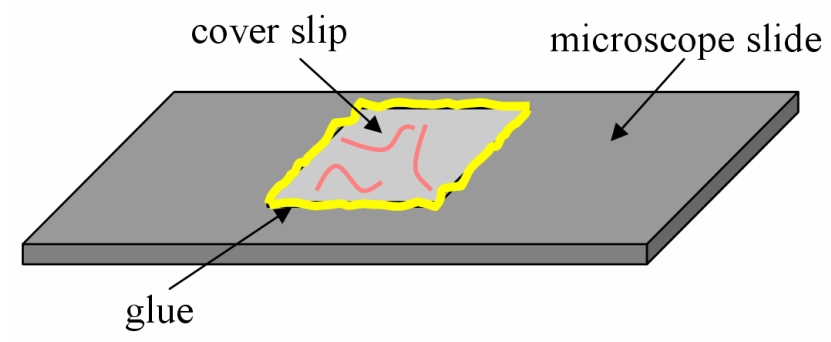

Figure 6-4: Actin Bundles in a quasi 2D-system.

The microscope slide and the cover slip are covered before use with a PEG layer (see chapter 2.2) to reduce sticking of the actin bundles at the glass surfaces. The actin bundles are confined in the height $(\approx 1 \mu \mathrm{m})$ but can fluctuate freely in the plane parallel to the microscope slide [36]. The fluctuations of the contour can be observed with fluorescence microscopy, because the actin bundles are always located in the focal plane. Thus, the actin bundles are investigated in a quasi 2-dimensional system, where the fluctuations in the height are negligible.
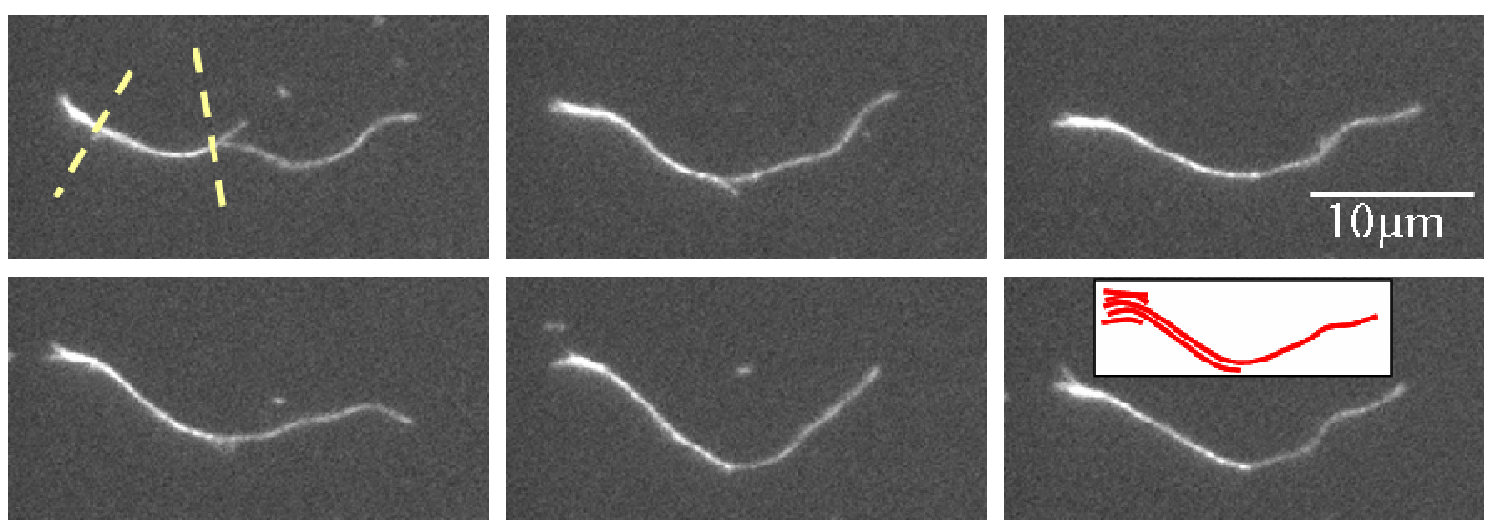

Figure 6-5: Fluctuating actin bundle (time period between the images: $\Delta t=240 \mathrm{~ms}$ ). This actin bundle consists of three parts: one thin end consisting of a single filament, a middle part consisting of two filaments and one thick end consisting of many filaments.

An example of a fluctuating actin bundle is shown in figure 6-5. By means of the intensity along the bundle, the bundle can be divided into three parts with approximately uniform intensity (see first image in figure 6-5). The thin end (right end in the images) is a single actin filament, the middle part consists of two actin filaments, and the thick end consists of several filaments. The fluctuations of the shape at the thin end are much larger than the fluctuations at the middle part or at the thick end. Obviously, the stiffness of bundles depends on their thickness. The intensity at different positions of the considered bundle is shown in figure 6-6. An increase of the intensity is obtained from a single filament to a thicker bundle. In the following, we want to use this effect to determine the number of filaments inside bundles. 


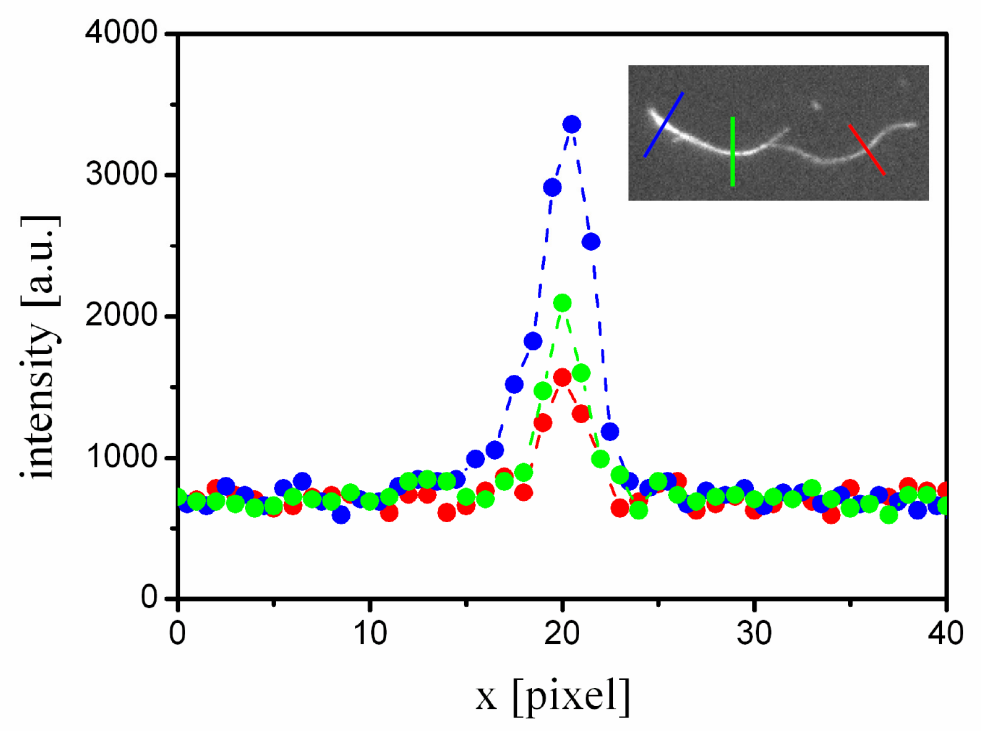

Figure 6-6: Intensity along lines at different positions (see inset) of the actin bundle.

For the analysis, the bundles are divided into parts with approximately uniform intensity. The different parts are treated as bundles consisting of a constant number of filaments along its contour. For each part of a bundle, the intensity is measured along lines perpendicular to the contour in distances of $0.5 \mu \mathrm{m}$ along the contour. The background is subtracted from the intensity profiles and the sum of the intensity is calculated for each profile. The sum of intensity is averaged over all measured intensity profiles in all recorded images. A parameter $I_{0}$ is obtained, which corresponds to the number of filaments inside a bundle.

The persistence lengths of bundles are determined by measuring the tangent correlation (see equation 3.3). A pixelline is created from the recorded image by using several image filters (see chapter 5.2). After smoothing the pixelline with a spline fit, the pixelline is divided into tangent vectors of equal lengths and the angles $\vartheta$ are measured between all tangent vectors. To obtain the tangent correlation $\langle\mathbf{t}(s) \mathbf{t}(s+l)>$ of two tangent vectors separated by a distance $l$, the cosine of the angle $\vartheta(l)$ is averaged over the measurements of all possible realization of the distance $l$ along the pixelline in one picture and over all recorded pictures for the same bundle. The spline fit for smoothing the pixelline and the length of the tangent vectors are optimized to reduce errors due to the "pixellization" of the contour (for details see [5]). 


\subsubsection{Persistence of Actin Bundles}

Figure 6-7 shows the tangent correlations for the middle part and for the thin end of the actin bundle seen in figure 6-5. The thick end of this bundle is too short and too stiff for a reasonable analysis of its fluctuations. The tangent correlations decay exponentially, which is also expected for a free semiflexible polymer modeled as a worm-like chain (see equation 3.3). From exponential fits, the persistence lengths $L_{p}$ are obtained. The persistence length $L_{p} \approx 25 \mu \mathrm{m}$ for the middle part is about two times larger than for the thin end, having $L_{p} \approx 14 \mu \mathrm{m}$. The persistence length of the thin end is in agreement with measurements of single actin filaments [5].

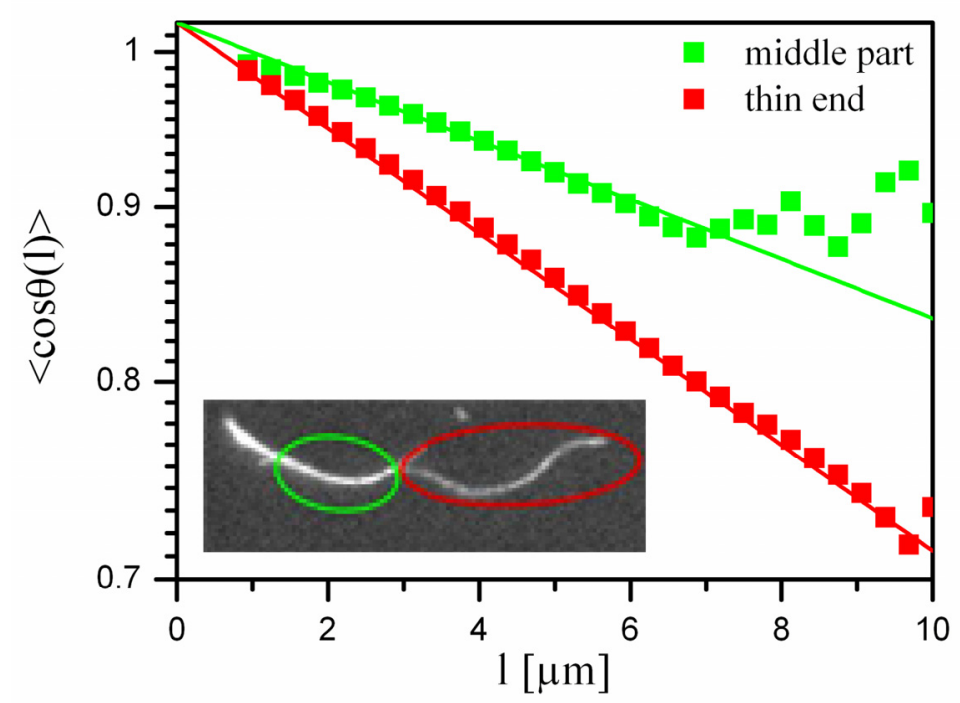

Figure 6-7: Tangent correlation (semi-logarithmic scale) of the actin bundle from figure 6-5.

The persistence lengths are determined for several bundles by measuring the tangent correlation, for which about 100 pictures per bundle are analyzed. Additionally, the intensities $I_{0}$ of the bundles are measured. With the same techniques, single actin filaments are also analyzed. We are limited with the analysis to bundles consisting of only a small number of filaments. For thick bundles, the thermal fluctuations are only very small due to the increased persistence lengths. Typically, actin bundles are only homogenous in their thickness at a length scale of a few micrometers. At this length scale, thick bundles behave like a stiff rod and a reasonable analysis of fluctuations to determine the persistence length cannot be performed.

In figure 6-8, the persistence lengths and intensities are shown for all analyzed bundles and filaments. The persistence lengths increase with larger intensities. Four accumulations of data points are found at $I_{0} \approx 4700, I_{0} \approx 7400, I_{0} \approx 9400$ and $I_{0} \approx 15100$. Apparently, we observed four different kinds of bundles. Data points for bundles at 


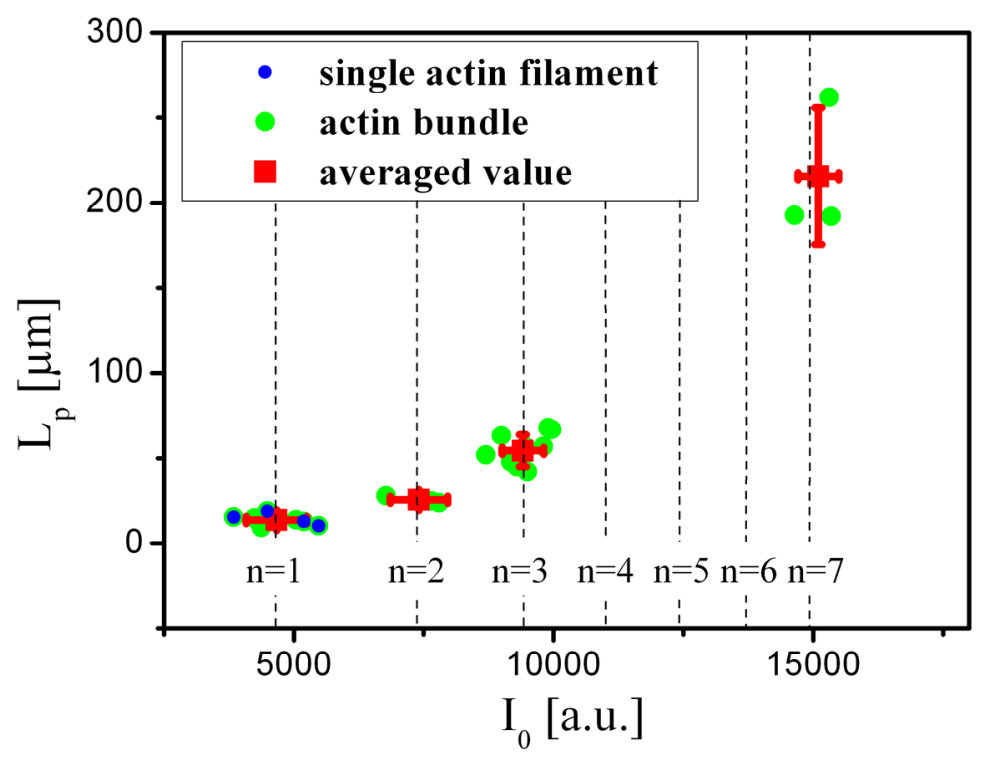

Figure 6-8: Persistence length of actin bundles against intensity.

$I_{0} \approx 4700$ agree in their intensities and persistence lengths with measurements of single actin filaments. Therefore, these data points can be related to parts of bundles consisting of a single filament. The data points at the next higher intensities $I_{0} \approx 7400$ and $I_{0} \approx 9400$ correspond to measurements of bundles consisting of two and three filaments. Comparing the distances between the data points, the intensity $I_{0} \approx 15100$ belongs to a larger number of filaments than four. Assuming that the difference in intensity for bundle consisting of $n$ and $n+1$ filaments decreases exponentially (see also section 6.4.2), the data points at $I_{0} \approx 15100$ correspond to bundles consisting of $n \approx 7$ filaments. After relating the intensity measurements with the number $n$ of the filaments, the persistence lengths are averaged for all data points with the same $n$. The obtained persistence lengths in dependence on number $n$ are shown in figure 6-9.

Bundles consisting of filaments between $n=3$ and $n=7$ are missing in the experiment. Additionally, bundles consisting of two filaments are found less often than single filaments and bundles consisting of three filaments. It is possible that these results only indicate that too few bundles were analyzed. However, it is also imaginable that bundles consisting of special numbers of filaments are more stable and that these bundles are found more frequently in solution. The filaments inside the bundles formed in the presence of divalent ions are close packed in a hexagonal order [58, 59]. The top layer of all favorite configurations $(n=1, n=3$ and $n=7)$ are completely filled with filaments, which might be a hint for a larger stability of these configurations. In order to support this hypothesis, further investigations with larger sampling sizes are necessary. 


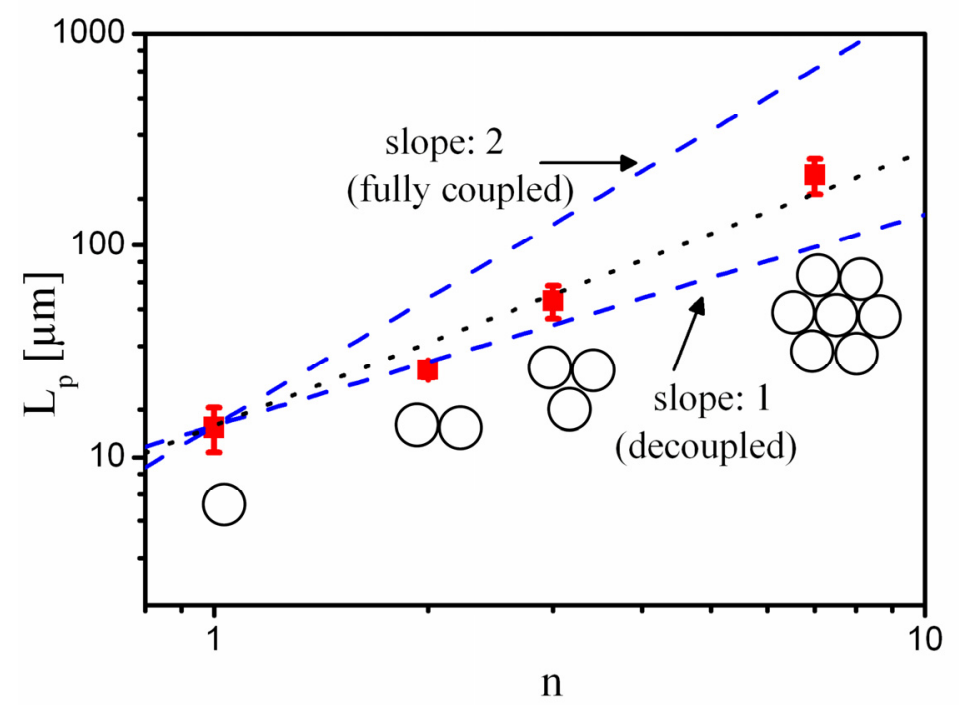

Figure 6-9: Persistence length of actin bundles against the number of actin filaments inside the bundle.

As seen in figure 6-9, the persistence length of actin bundles increases strongly with the number of filaments. For comparison, the two limited cases, $L_{p} \propto n$ (see equation 6.5) for decoupled filaments and $L_{p} \propto n^{2}$ (see equation 6.9) for fully coupled filaments, are drawn. The measured values lie in an intermediate regime and the filaments are neither decoupled nor fully coupled. It seems that filaments are partly coupled with a persistence length of $L_{p} \propto n^{1.3}$. This coupling is surprising, because no linker molecules crosslink two filaments directly by binding to both filaments for bundles formed by counterion condensation. Thus, no linker molecules have to be stretched for a sliding of actin filaments against each other, and the decoupled case could be expected. However, this is not the case and the binding of the actin filaments leading to the bundling has to be considered. The actin filaments in bundles formed by counterion condensation are held together by electrostatic interactions. One model used to explain actin bundling is based on thermal fluctuation correlation effects between 1D Wigner crystals of counterions condensed on filaments $[136,137]$. In figure $6-10$, the charge distribution of a bundle consisting of two filaments is considered. For a straight bundle (figure 6-10a), the charges are ordered in a lattice that always has the same distance between neighboring condensed charges. In contrast, some charges have an unfavorable distance to neighboring charges (figure 6-10b) for a bent bundle in which the filaments are bent independently (decoupled case). To avoid this, an additional stretching or compression of the filaments is necessary. This leads to an indirect coupling of the filaments, which explains the partly coupled behavior. For actin bundles formed by multivalent ions, other effects are also observed which point to deformations of actin filaments inside 
bundles in order to avoid an unfavorable charge distribution. In rings of actin bundles, the contour is not smooth like in bundles formed by ABPs or depletion forces. Kinks are found that are also explained by optimizing the charge distribution inside the actin bundles [136]. Furthermore, the helical actin filaments inside the bundles are twisted due to electrostatic interactions [58,61].

a)

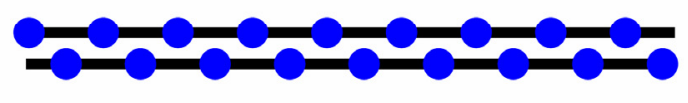

b)

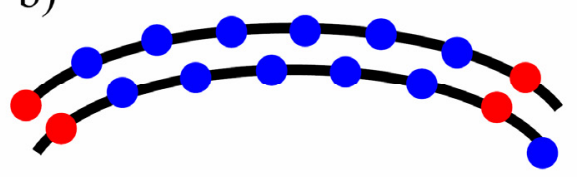

Figure 6-10: Charge distribution of actin bundles. a) For a straight bundle, a lattice of charges is found. b) In a bent bundle, some neighboring charges (red color) have an unfavorable distance.

The partly coupled behavior measured for bundles formed by counterion condensation is not universally valid for all actin bundles. Actin bundles formed by ABPs or depletion forces can show a different behavior due to other types of interactions that lead to bundling. Recent experiments show that the persistence length depends on properties and concentration of the linker molecules for actin bundles formed by ABPs [67]. The decoupled case, the fully coupled case, and also a partly coupled case is measured for different conditions [67]. Actin bundles formed by depletion forces show a fully coupled behavior [67].

In summary, the persistence of actin bundles formed by multivalent ions was investigated in this section. Intensity measurements of the fluorescently labeled actin filaments allow us to identify the number of filaments inside the bundles. Analyzing the thermal fluctuations of the bundles by the tangent correlation, the persistence length of each bundle can be determined. Considering several bundles, the persistence length in dependence on the number of filaments is obtained. The results suggest a partly coupled behavior of the actin filaments inside the bundles. This coupling is probably due to an optimization of the charge distribution inside the bundles.

After characterizing the mechanical behavior of actin bundles, the evolution of the actin bundling is investigated in the next section. Whereas in this section multivalent ions are used for the formation of actin bundles, we change the linker molecules for subsequent investigations. In the following section, we use the ABP $\alpha$-actinin. Aside from the larger relevance for biology systems, the advantage of using $\alpha$-actinin is the possibility to label them with a fluorescence dye in order to perform FRET experiments (see section 6.4.3). 


\subsection{Bundling Kinetics}

\subsubsection{Hydrodynamic Focusing}

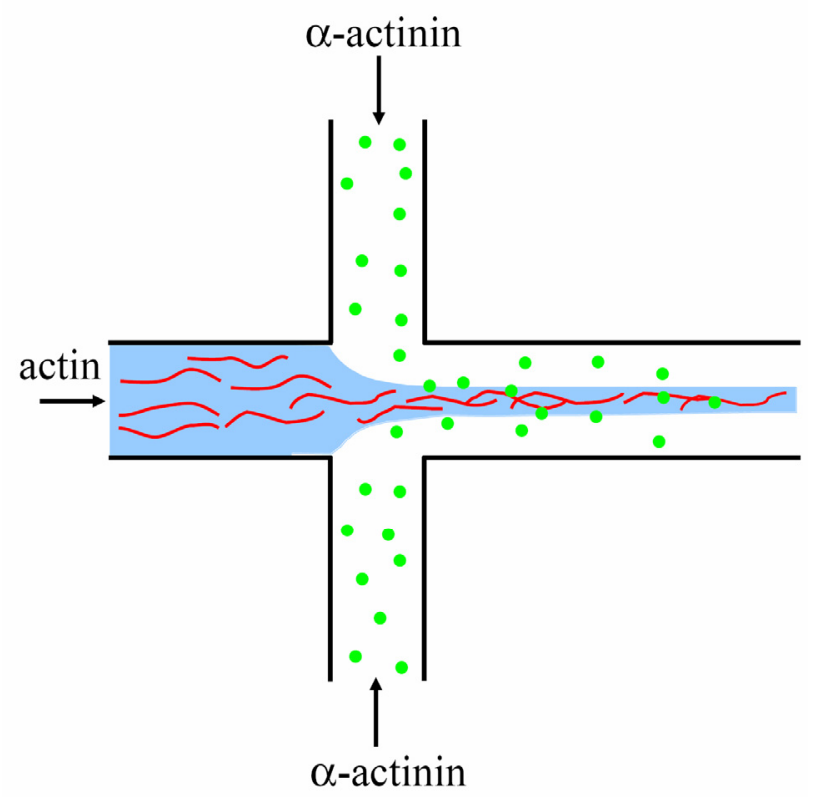

Figure 6-11: Formation of actin bundles in a hydrodynamic focusing device.

To study the time-resolved formation of actin filaments into bundles, we use a hydrodynamic focusing device [138] consisting of a crossed channel geometry (see figure 6-11). The solution in the main channel is hydrodynamically focused by the fluids from two side channels. The mixing of the fluids in the outlet channel occurs only by diffusion due to laminar flow inside the microchannels (small Reynolds numbers). The width of the center stream in the outlet channel can be adjusted by the velocities of the side channels relative to the velocity of the main channel. If all velocities inside the inlet channels are the same, the center stream occupies one third of the volume of the outlet channel. For larger velocities in the side channels relative to the main channel, the center stream becomes narrower. Concentrations of the fluids inside the outlet channel can be controlled by adjusting the width of the center stream.

Hydrodynamic focusing devices are often used to study the reaction of two solutions by diffusive mixing [139-142]. Usually, a solution containing the first reagent is injected into the main channel and a solution containing the second reagent is injected from the side channels. Measuring different positions along the outlet channel, the evolution of the reaction can be analyzed at different times.

To study the formation of actin bundles, a solution with actin filaments (70nM) is injected into the main channel (width: $30 \mu \mathrm{m}$, depth: $20 \mu \mathrm{m}$ ). From both side channels 
(width: $30 \mu \mathrm{m}$, depth: $20 \mu \mathrm{m}$ ), an aqueous solution containing the actin-binding protein $\alpha$-actinin $(10 \mathrm{nM})$ is injected. The velocities $\left(\bar{v}_{i n}=255 \mu \mathrm{m}\right)$ in all inlet channels are the same. Inside the outlet channel (width: $50 \mu \mathrm{m}$, depth: $20 \mu \mathrm{m}$, length: $\approx 0.7 \mathrm{~m}$ ), the fluids velocity is of $\bar{v}=450 \mu \mathrm{m} / \mathrm{s}$. Actin filaments are imaged at different positions along the outlet channel by using fluorescence microscopy. We know from measurements of the center-of-mass of actin filaments inside microchannels (see chapter 5.5) that most filaments are not found in the center of the channels. Therefore, we focused the microscope to a plane $8 \mu \mathrm{m}$ below the upper (glass) surface of the microfluidic device. This has also the advantage that the actin filaments are mainly stretched and aligned (see chapter 5.4) and fluctuations out of the focal plane are reduced.

\subsubsection{Evolution of Actin Bundling}

Figure 6-12a shows typical recorded pictures at different positions along the outlet channel. The intensities of lines drawn through the bundles, which are a measure of the number of filaments inside the bundles, are seen in figure 6-12c. At the beginning of the outlet channel $(x=0 \mathrm{~cm})$, the observed macromolecules are single actin filaments. Along the outlet channel, the filaments form bundles and the bundles grow in their thickness.

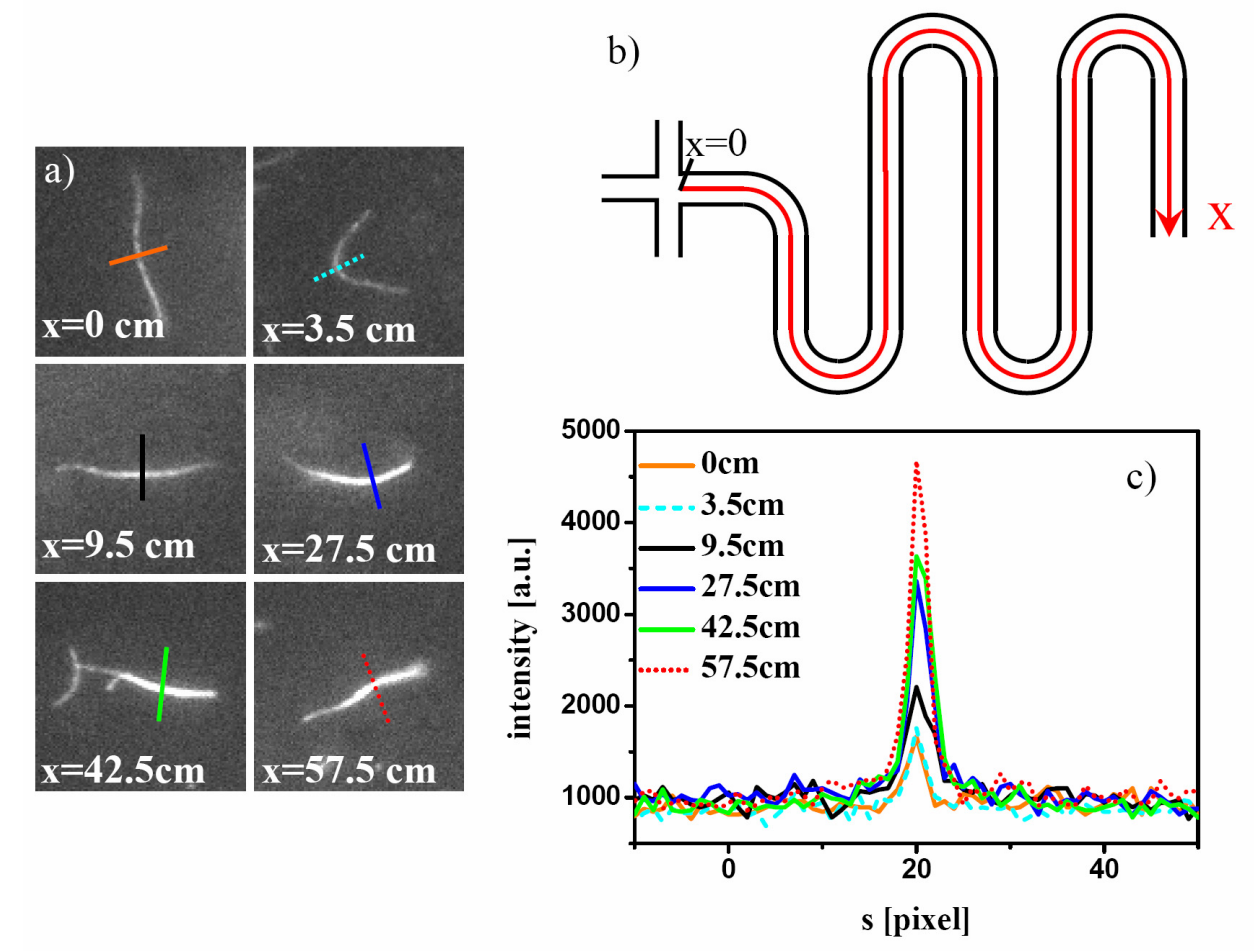

Figure 6-12: Formation of actin bundles in a hydrodynamic focusing device. a) Snapshots at different positions along the outlet channel. b) Sketch of the microfluidic device. c) Fluorescence intensity of the actin bundles. 
Using the known velocity $\bar{v}$ in the outlet channel, measurements at a position $x$ can be also considered as observation of the bundling process at a time $\bar{t}=x / \bar{v}$. We start the measurement at $\bar{t}=1.3 \mathrm{~min}$ for which the most of the filaments are still in the state of single filaments. At $\bar{t}=1.3 \mathrm{~min}$, the filaments are spread over the entire channel width by diffusion and migration. The diffusion of $\alpha$-actinin (small molecules: length about $40 \mathrm{~nm}$ ) is much faster than for actin filaments and they are already homogenously distributed after about 20s. Therefore, we start the measurements at a time where both solutions are mixed completely.

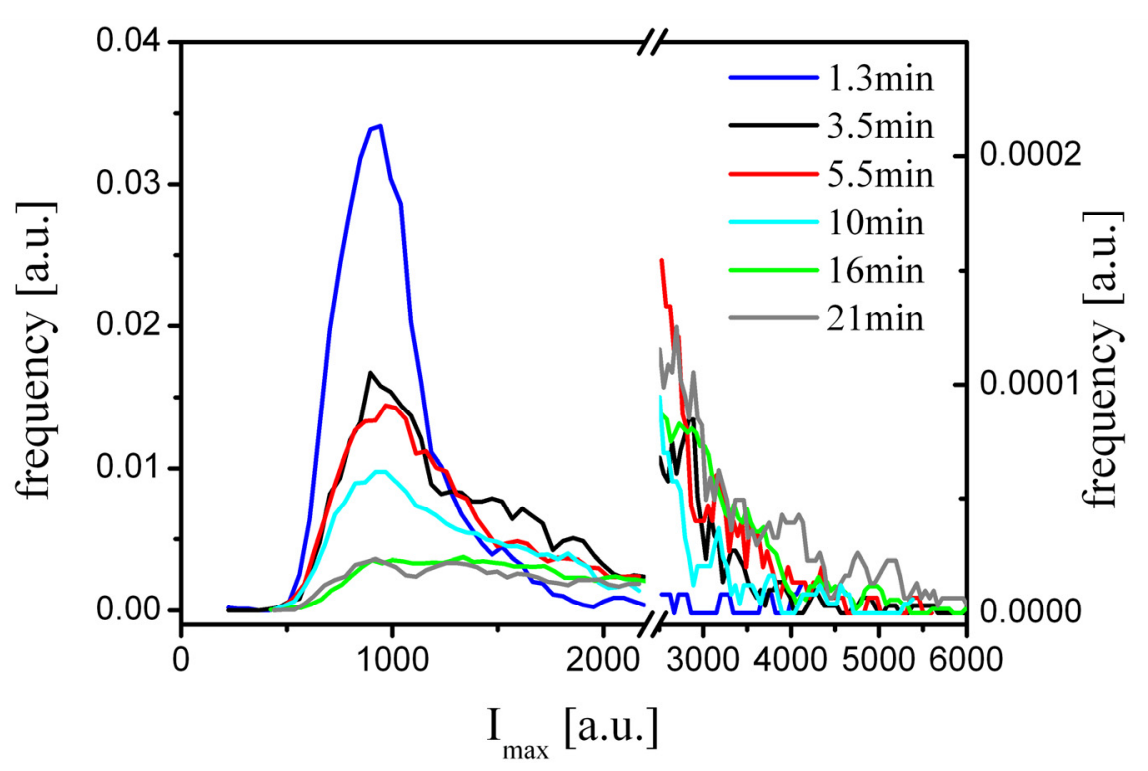

Figure 6-13: Intensity probability distributions at different times.

For different times, the maximal intensity $I_{\max }$ of each bundle relative to the background is measured in the recorded images. Around 1000 bundles are analyzed for each time. The intensity scale is subdivided into intervals of $\Delta I_{\max }=48$ for which the numbers of bundles with corresponding $I_{\max }$ are counted. The obtained intensity probability distribution at a time $\bar{t}$ is normalized by the total number of analyzed pictures. Figure 6-13 shows the intensity probability distributions at different times. The distributions have a maximum at $I_{\max } \approx 900$. The peak heights of these maxima, which are a measure for the number of single filaments inside the solution, decrease with increasing time. Therefore, less single actin filaments are observed at later times. Comparing the two distributions for smallest times ( $\bar{t}=1.3 \mathrm{~min}$ and $\bar{t}=3.5 \mathrm{~min}$ ), the maximum at $I_{\max } \approx 900$ is clearly smaller at $\bar{t}=3.5 \mathrm{~min}$ and instead the intensities $I_{\max }>1400$ are more frequently. Some filaments, which are detected at $\bar{t}=1.3 \mathrm{~min}$ still as single filaments, are bound together and form bundles consisting of a few filaments. For later 
times, the frequencies in the intensity range of $I_{\max } \approx 1400-2000$ decrease again and the frequencies get instead larger at higher intensities. This indicates an increase of the number of filaments inside the bundles. Thus, filaments firstly organize into bundles and the numbers of filaments forming these bundles increase with time, as expected.
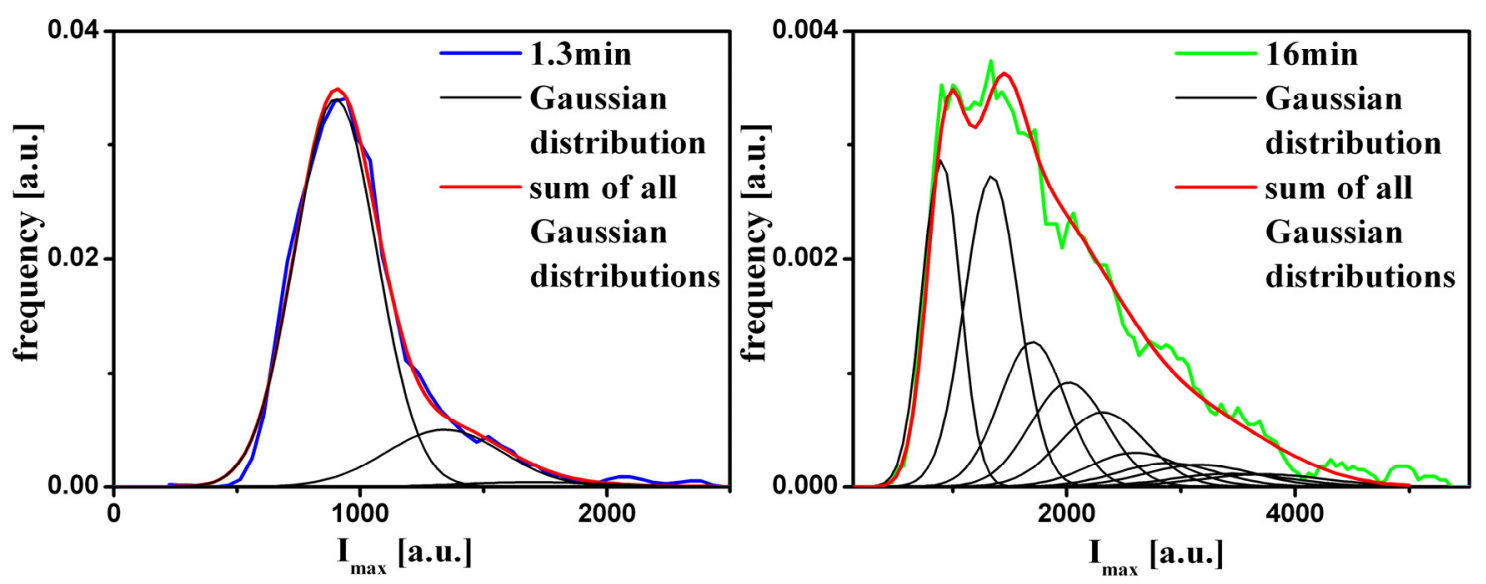

Figure 6-14: Gaussian fits of the intensity probability distributions.

To determine the frequency of bundles consisting of $n$ filaments at different times, the intensity probability distributions are fitted by a sum of Gaussian distributions, where the $n$-th Gaussian distribution corresponds to $n$ filaments ( $n=1$ : single filaments, $n=2$ : bundle consisting of two filaments, etc.). In figure 6-14, such Gaussian fits are shown for two different times. The positions and widths of the Gaussian distributions are kept constant for all fits at the different times. The positions $\left(I_{1}=900\right.$ and $\left.I_{2}=1341\right)$ of the first and second Gaussian distributions and its widths $\left(w_{1}=334\right.$ and $\left.w_{2}=472\right)$ are obtained by fitting the intensity probability distribution for $\bar{t}=1.3 \mathrm{~min}$ with two Gaussian distributions. The width of the $n$-Gaussian distribution is assumed to increase with number of filaments inside a bundle with $w_{n}=\sqrt{n} w_{1}$. The positions of the 3 th-5th Gaussian distributions are determined by the fits of the distributions for $\bar{t}=3.5 \mathrm{~min}$ and $\bar{t}=5.5 \mathrm{~min}$. Due to the increasing width of the Gaussian distributions and therewith the increasing overlap of neighboring distributions, the positions at larger numbers $(n>5)$ cannot be determined anymore. In these cases, the decrease in distance between neighboring Gaussian distributions, which is obtained for small numbers, is continued exponentially. The area under the $n$-th Gaussian distribution corresponds to the frequency of bundles consisting of $n$ filaments inside the solution. The frequency distributions of the number $n$ at different times are shown in figure 6-15. 


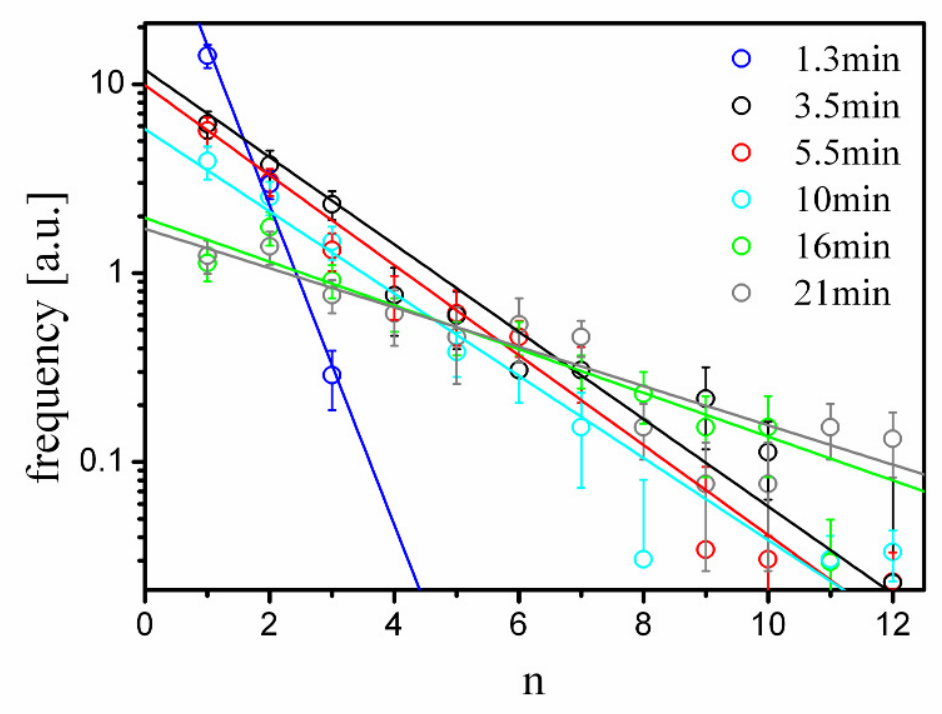

Figure 6-15: Frequency distributions of the number of filaments inside bundles at different times.

To quantify the time evolution of the bundling process, we assume a step-growth process modeled similar to a step-growth polymerization [143]. As the name already implies, the formation of thick bundles is considered by a series of steps. An example of a possible formation from single filaments to a thick bundle is drawn in figure 6-16. The actin filaments are considered as rods, each having the same length. Each filament and each bundle is allowed to bind to other bundles/filaments and the number of filaments inside the resulting bundle is the sum of all involved filaments:

$$
\begin{aligned}
& A_{1}+A_{1} \rightarrow A_{2} \\
& A_{1}+A_{2} \rightarrow A_{3} \\
& A_{1}+A_{3} \rightarrow A_{4} \\
& A_{2}+A_{2} \rightarrow A_{4} \\
& \ldots \\
& A_{n}+A_{m} \rightarrow A_{n+m} \quad n, m=1,2, \ldots
\end{aligned}
$$

where $A_{n}$ is a bundle consisting of $n$ filaments.

Indeed in reality, the actin bundling is more complicated as the reaction equations 6.10 in this model can describe. Typically, the thickness of a bundle is not homogenous along the contour. The number of filaments inside the bundle can vary along its contour and is not necessarily the sum of all actin filaments inside the entire bundle. For example, two short actin filaments can bind to a long filament in the way that a bundle consisting of only two filaments is generated (see figure 6-16b). In addition, bundles grow not only in thickness, but also in length [144, 145] (see figure 6-16c). But experiments show [144, 145], that the increase of the length is a slow process in 


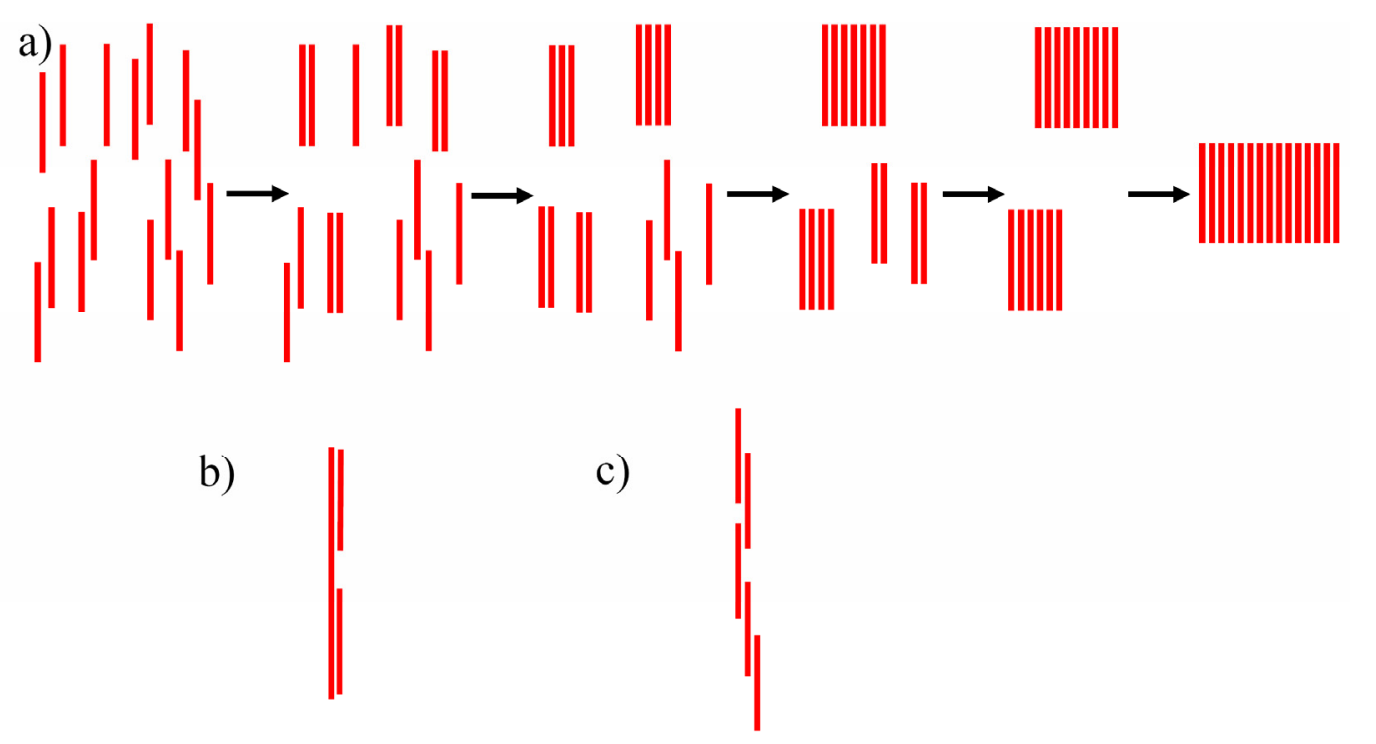

Figure 6-16: Step-growth process. a) Possible formation of an actin bundle step by step. b)-c) Possible configurations of filaments inside actin bundles which are observed in experiments but not included into the model.

comparison to the increase of the thickness of bundles. We measure the bundling of actin filaments at relatively small times. Therefore, it can be expected that the main process is indeed the increase of the thickness as described in the step-growth model. Assuming that all bundles and all single filaments bind to other bundles and filaments with the same probability at a time $t$, the bundling can be considered as a second-order reaction in which two bundles/filaments reacts to a thicker bundle:

$$
A+A \stackrel{k}{\rightarrow} A,
$$

where $A$ denotes a bundle or a single filament and $k$ is the rate constant. According to this reaction equation, the concentration $[A]$ of the sum of bundles and single filaments inside the solution at a time $t$ is determined by the rate law [143]

$$
\frac{d[A]}{d t}=-k[A][A] .
$$

The solution of this differential equation is given by [143]

$$
[A]=\frac{[A]_{0}}{1+k[A]_{0} t},
$$

where $[A]_{0}$ is the initial concentration of single filaments.

Describing the bundling as a reaction for which each filament has exactly one binding site, the reaction can be considered as the attaching of bundles/filaments at free binding sites of other bundles or filaments. $(n-1)$ reactions are needed for formation of a bundle 
consisting of $n$ filaments. The bundle contains $(n-1)$ reacted filaments ( $\triangleq$ filaments whose binding sites are occupied form other filaments) and one unreacted filament ( $\triangleq$ filament with free binding site). Therefore, each bundle and each single filament has exactly one unreacted filament, and the concentration of unreacted filaments is equal to the concentration $[A]$ of the sum of bundles and single filaments. The probability for a bundle consisting of $n$ filaments is equal to the probability that $(n-1)$ reactions have occurred and one filament has not reacted. If $p$ is the probability for binding one single filament or bundle to another filament or bundle until the time $t,(1-p)$ is the probability for an unreacted filament. Thus, the probability $x_{n}$ of a bundle consisting of $n$ filaments is given by

$$
x_{n}(p)=p^{n-1}(1-p) .
$$

The probability $p$ can be expressed by the fraction of filaments which have reacted at anytime until the time $t$. The concentration of reacted filaments can be calculated by the difference of the concentration $[A]_{0}$ of all single filaments in the beginning of the experiments and the concentration $[A]$ of unreacted filaments. Using equation 6.13 for the concentration $[A]$, the probability $p$ leads to

$$
p(t)=\frac{[A]_{0}-[A]}{[A]_{0}}=\frac{k[A]_{0} t}{1+k[A]_{0} t} .
$$

The probability $p$ is zero at the beginning, increases with time, and becomes one at $t=\infty$. In the experiment, the frequency distribution of the number of filaments inside a bundle at a time $\bar{t}$ is measured (see figure 6-15). The measured frequency of a bundle consisting of $n$ filaments is proportional to the probability $x_{n}$ that a bundle with this number is formed. The logarithm of $x_{n}$

$$
\ln x_{n}=n \ln p-\ln p+\ln (1-p)
$$

is a linear function in $n$ with a slope depending on $p$. Fitting the logarithm of the frequency distribution at a time $\bar{t}$ linearly, the probability $p(t)$ can be calculated from the slope. The results at different times are shown in figure 6-17. As expected, the probability increases with time. The data points are fitted with a function $p(t)=c t /(1+c t)$, where the fitting parameter $c$ is related with the rate constant $k$ by $c=k[A]_{0}$ (see equation 6.15). As a result, $c=4 \cdot 10^{-3} \mathrm{~s}^{-1}$ is obtained. The initial concentration $[A]_{0}=0.8 \cdot 10^{-12} \mathrm{M}$ of actin filaments is estimated by the averaged number $N_{0}=0.36$ of actin filaments inside the considered volume $27.5 \mu \mathrm{m} \times 27.5 \mu \mathrm{m} \times 1 \mu \mathrm{m}$ (focal depth about $1 \mu \mathrm{m}$ ) in one picture at a time $\bar{t}=1.3 \mathrm{~min}$. This leads to a rate constant for the bundling process of $k=5 \cdot 10^{9} \mathrm{~s}^{-1} \mathrm{M}^{-1}$. 


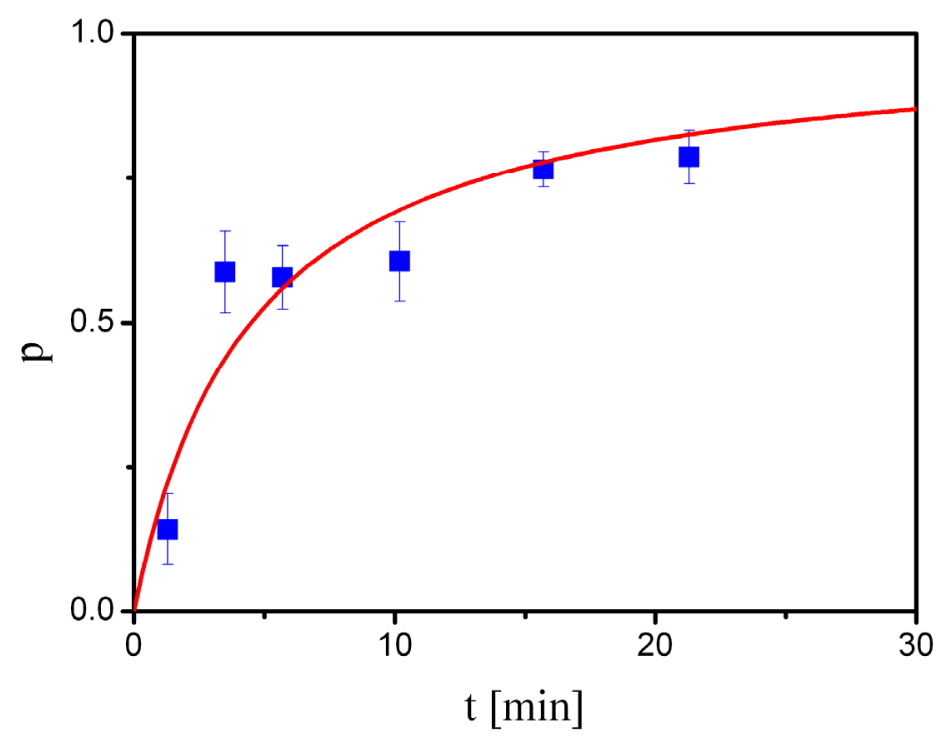

Figure 6-17: Probability of binding one filament/bundle to another filament/bundle.

The measured rate constant should be considered as an estimation of how fast the bundling process occurs. Aside from simplifications in the model, an experimental problem complicates the interpretation of the measurements. At late times, a larger amount of short actin filaments is observed than in the beginning of the experiment. It seems that actin filaments somehow break and the total number of filaments increases during the bundling process. Perhaps, ends of only partly-bound filaments break away from the bundles due to the shear in the microchannels. It is also possible that the shortening of actin filaments is a general mechanism involved in the actin bundling, as reported for measurements under quite different conditions [146, 147]. For further experiments, it would be helpful to prevent the shortening of actin filaments or to quantify the increase and include it into the model to obtain more precise information about the time evolution of the bundling process. Although improvements of the experiment and model promise a better understanding of the bundling process, the order of magnitude of the measured rate constant in this experiment already gives us the time scale for the actin bundling.

For diffusion-controlled reactions, the rate constant is limited by the time which is needed to bring the reactants together by diffusion. Assuming that two reactants react immediately after bringing them together, the rate constant for ellipsoidal molecules is given by [148]

$$
k_{\text {diff }}=4 \pi D N_{A} \frac{a}{\ln (2 a / b)},
$$

where $D$ is the diffusion constant, $N_{A} \approx 6 \cdot 10^{23} \mathrm{~mol}^{-1}$ is the Avogadro constant, and $a$ is the major and $b$ the minor semi-axis of the ellipsoid. Using the diffusion constant 
$D=k_{b} T(\ln (2 a / b)+0.5) / 8 \pi \eta a$ [81] of ellipsoids perpendicular to the main axis (the actin filaments/bundles are mainly aligned in flow direction), the rate constant is simplified to

$$
k_{\text {diff }}=\frac{N_{A} k_{b} T}{2 \eta}\left(1+\frac{1}{2 \ln (2 a / b)}\right) .
$$

Considering the actin filaments (length $\approx 8 \mu \mathrm{m}$ ) as ellipsoid with semi-axes $a \approx 4 \mu \mathrm{m}$ and $b \approx 44 \mathrm{~nm}$ (assuming that the actin filaments are covered completely with $\alpha$-actinin having a length of $40 \mathrm{~nm}$ ), the diffusion-controlled rate constant leads to $k_{\text {diff }}=1.4 \cdot 10^{9} \mathrm{~s}^{-1} \mathrm{M}^{-1}$. This is in the same order of magnitude as the measured rate constant. Thus, the bundling of actin filaments is a diffusion-controlled process. Actin filaments which are brought close enough together by diffusion bundle almost immediately and diffusion is the limiting factor for the velocity of the bundling process.

\subsubsection{Outlook}

We showed with our experiment the possibility to investigate the time evolution of a bundling process by using microfluidic tools. Intensity measurements of the emitted light from the fluorescently labeled filaments determined the number of filaments inside bundles. Measurements at different channel positions in a hydrodynamic focusing device, which correspond to measurements at different times of the bundling process, allowed the observation of the time-depending bundling states, starting with single filaments and resulting in thick bundles. Using a step-growth model, a rate constant for the bundling was determined which shows that actin bundling is a diffusion-controlled process.

The description of a step-growth process for the actin bundling is a simplification which in future may be improved to gain deeper insights into the time evolution. Actually, we already see effects due to slight deviations from the model in which is assumed that all bundles as well as all single filaments can bind to each other with the same probability to form thicker bundles. With this assumption, the most molecules in the solution should always be single filaments, even at very large times. But it can be seen in figure 6-15, that for late times the single filaments are found less frequently than bundles consisting of two filaments. Thus, the probability to bind to a bundle must be larger in comparison to the probability to bind to a single filament. It is also imaginable that actin bundles with special numbers of filaments are more likely formed as it is already speculated in section 6.3.2. To understand such details, further experiments are needed. Modification of the experiment such as changes in actin concentration and/or $\alpha$-actinin concentration could lead to additional aspects of the bundling process. Furthermore, the 
use of different linker molecules could show similarities and special features from different kinds of actin bundles.

So far, we imaged only one component, the actin filaments, involved in the bundling. No attention is paid to the linker molecules and the interactions leading to bundling are not concrete in the model. FRET (Fluorescence Resonance Energy Transfer) microscopy can help to obtain a better understanding of the interactions inside the bundles. This technique is based on a radiationless energy transfer between two dye molecules which have an overlap in the emission spectrum of the first dye and the absorption spectrum of the second dye. The first dye is excited by an external illumination of the sample with wavelength according to its absorption spectrum. Instead of emission of photons, for small distances (typically $<10 \mathrm{~nm}$ ) the energy of the exited states of the first dye is transferred by long-range dipole-dipole interactions to the second molecule and excites its state. Then, light from the second dye molecule is emitted. Information about the distance between the molecules can be determined by measuring the intensity of the emitted light from the first dye as well as from the second dye.

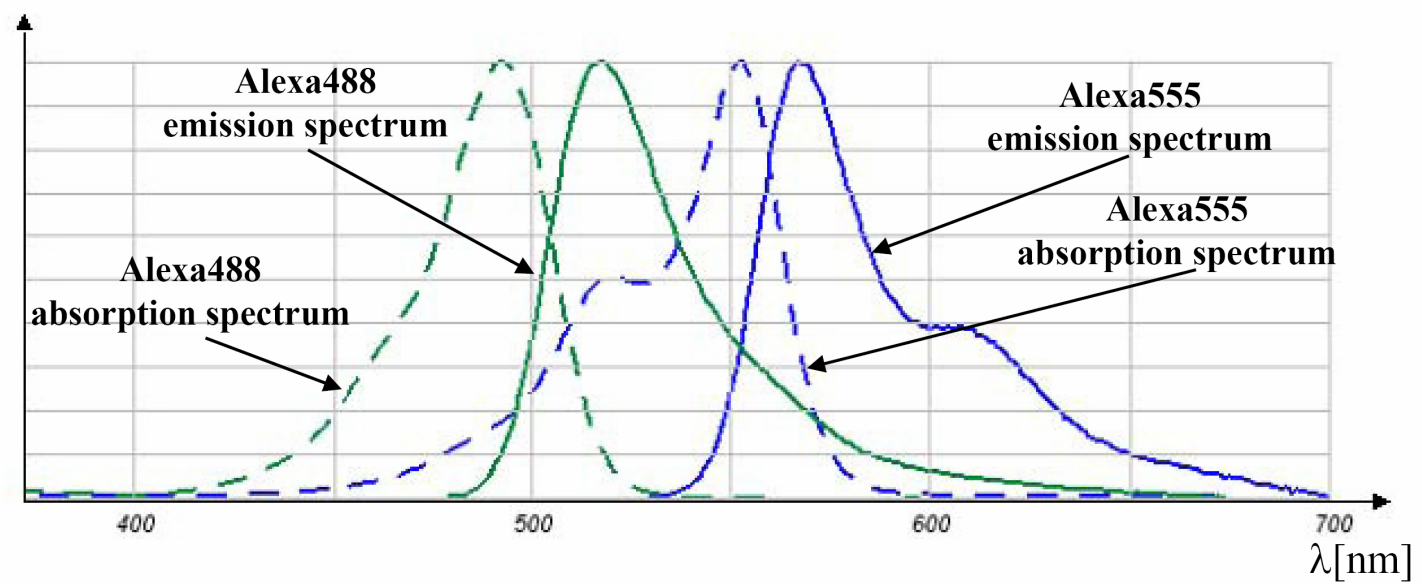

Figure 6-18: Emission and absorption spectra of Alexa488 and Alexa555 [149].
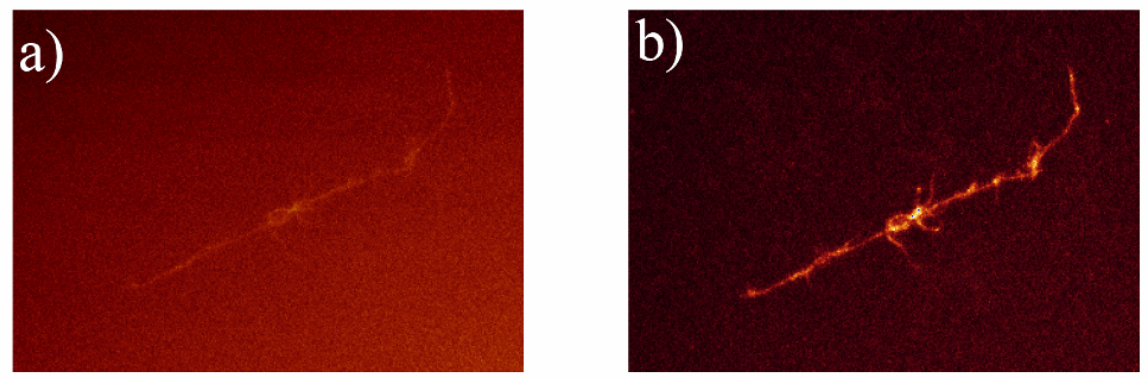

Figure 6-19: FRET microscopy of an actin bundle illuminated with a laser at 488nm and imaged at a) $495-515 \mathrm{~nm}$ and at b) $616-665 \mathrm{~nm}[149]$. 
Preliminary experiments with actin filaments labeled with Alexa488 and $\alpha$-actinin labeled with Alexa555 (both dyes purchased from Molecular Probes, Carlsbad, US) show promising results. The absorption and emission spectra for both dyes are shown in figure 6-18. A solution of actin bundles is illuminated at a wavelength of $488 \mathrm{~nm}$. At this wavelength, Actin-Alexa488 is excited, whereas the fraction of excited $\alpha$-ActininAlexa555 is negligible. In figure 6-19, two images of the same actin bundle recorded at different wavelengths with a confocal microscope (Leica Microsystems GmbH, Wetzlar) are shown. The contrast between the bundle and the background is weak and the bundle is hardly visible for $495-515 \mathrm{~nm}$ (see figure 6-19a), whereas the intensity of the bundle is strong for 616-665nm (see figure 6-19b). Considering the emission spectra for both dyes, the measured light at $495-515 \mathrm{~nm}$ is mainly emitted from the Actin-Alexa488 and the measured light at $616-665 \mathrm{~nm}$ from $\alpha$-Actinin-Alexa555. Thus, the emitted light of Actin-Alexa488 (which is exited by the external light source) is small compared to the emitted light of $\alpha$-Actinin-Alexa555. Consequently, an energy transfer from ActinAlexa488 to $\alpha$-Actinin-Alexa555 has occurred. This indicates the system is suitable for FRET microscopy. 


\section{Chapter 7}

\section{Conclusions}

Polymer and protein solutions are involved in many microfluidic applications for which knowledge about their behavior in microflow is helpful in dealing with these liquids. In order to further our understanding of polymer properties in microflow, the behavior of actin filaments in pressure-driven flow has been discussed in this thesis. Actin filaments provide an ideal platform to study the properties of semiflexible polymers. The results presented in this thesis are therefore relevant from a fundamental point of view. Additionally, actin filaments play an important biological role in cellular motility and mechanical stability of cells. Consequently, the organization of filaments in bundles and networks is of significant relevance. In the second part of this thesis, the properties of actin bundles and kinetics of the formation process were considered in vitro. To this end, a method to observe the actin bundling at a molecular scale in a time-resolved manner has been developed.

Fluorescently labeled actin filaments were observed using fluorescence microscopy which enables the visualization of the filament contours. A laser setup was built that facilitates short exposure times in order to avoid blurring of the contours of fast flowing filaments. Using stroboscopic illumination, dynamics of filaments in short time periods can be observed by illuminating the sample several times during a single image exposure.

The analysis of the contours of filaments in flow inside microchannels provided information about the conformational, orientational, and transport properties of semiflexible polymers. Experiments were performed with a dilute actin solution in order to investigate single polymer dynamics. Microchannels were used with aspect ratios (depth/width) of unity. The channel widths had almost the same size as the characteristic lengths of the polymers (contour length and persistence length). At this length scale, effects due to the semiflexible nature of actin filaments are predominant. The orientations and conformations of the filaments in flow were characterized by 
measuring the orientational order parameter, the angle probability distribution, and the end-to-end distance probability distribution. The results showed that the flow mainly elongated and aligned the filaments. The averaged alignment increased toward the channel walls. This was correlated to the increase of the local shear rate from zero at the centerline to maximal values at the walls. Additionally, the alignment and elongation increased for larger flow velocities. The characteristic parameters of the filament orientation, the preferred angle and the width of the angle probability distribution, obeyed scaling laws which are known for stiff or strongly-elongated polymers in simple shear flow. However, the filaments were not always in an elongated state and the contours changed from time to time. For example, fully-elongated filaments lying on a single streamline relaxed because of the absence of an elongational force. Mostly, the relaxed filaments elongated again. But sometimes, tumbling of filaments was observed. Furthermore, a particularity in conformation was found in the channel center due to the non-monotonic, parabolic velocity profile. In this region, stable bent parabolic shapes of filaments were observed. The bent contours of the tumbling filaments as well as of the parabolically bent filaments were described sucessfully by modeling the filaments as elastic rods. Balancing drag forces and bending forces, a scaling law was derived with which the measured decrease of the radii of curvature of the tumbling filaments with larger velocities was quantified. Moreover, the parabolic contours at the channel center were described.

In addition to conformational and orientational studies, the frequencies of filaments at different channel positions along a cross-section were examined. Whereas filaments for small velocities were equally distributed almost over the entire channel width, filaments for large velocities were most frequently observed approximately at the halfway points between the channel center and the channel walls. The center-of-mass probability distributions for large velocities showed local minima at the channel center. This striking minima became more pronounced at larger velocities. Furthermore, depletion layers near walls were found for which the thicknesses increased with larger velocities. These observations were explained by kinetic arguments. Spatially-varying diffusivity causes cross-streamline migration away from the channel center, leading to minima in the distributions. Hydrodynamic interactions with the walls generate a microflow which moves filaments away from walls as well and thereby creates depletion layers. Additionally, steric interactions with walls influence the depletion layers, in particular for small velocities. To characterize the thermal fluctuations of semiflexible actin filaments inside microchannels, segment distributions at different channel positions as well as for different velocities were analyzed. Assuming proportionality between the filament diffusivity and the mean square deviation of filament segments from the 
center-of-mass streamline, the dependence of the filament diffusivity on the shear rate was determined. Including this diffusivity and hydrodynamic interactions with walls in the governing equation of the center-of-mass probability distributions, they were calculated analytically for different velocities. The calculations showed the same essential characteristics as the measurements. For large velocities, a nearly quantitative agreement was even obtained. First measurements and calculations in a curved geometry suggested that center-of-mass probability distributions become asymmetric, and in particular the local minimum is shifted toward the inner wall.

The second part of this thesis considered the bundling of actin filaments by counterion condensation in the presence of $\mathrm{Ca}^{2+}$ or by the actin-binding protein $\alpha$-actinin. A method was developed to time-resolved measure the formation from single filaments to thick bundles at a molecular scale. The numbers of filaments inside bundles were determined by intensity measurements of the emitted light from fluorescently labeled filaments. Using a hydrodynamic focusing device, the bundle states at different times during the bundling process were studied in situ. Describing the bundling by a stepgrowth model, a rate constant was determined which showed that the bundling is a diffusion-controlled process. Furthermore, the analysis of thermal fluctuations characterized the mechanical properties of bundles. The persistence lengths were obtained which increased with larger numbers of filaments. The results suggested a weak coupling between filaments, which is probably due to an optimization of the charge distribution inside bundles.

The results presented in this thesis contribute to the understanding of complex fluids in microfluidic as well as of biological systems. Knowledge about the behavior of semiflexible polymers inside microfluidic channels can be useful for many microfluidic applications. For example, information about the channel positions facilitates their detection and manipulation. Based on the results, new techniques such as for the analysis or separation of polymers might be developed. Since flow at the microscale is omnipresent in biological systems, the results are additionally of fundamental biological interest. Similar kinds of problems as presented in this thesis are found inside the body such as the flow of particles, cells, and polymers inside blood vessels. Furthermore, first steps were performed to investigate the kinetics of the actin bundling by using microfluidic tools. Further studies are necessary for deeper insights and we propose to use FRET microscopy in order to gain more information about the interactions inside the bundles during their formation. Aside from actin bundling, the concepts and methods might be also used to time-resolved study the formation of actin networks as well as the organization of other biological systems. 


\section{Chapter 8}

\section{Appendix}

\subsection{Calculation of the Center-of-Mass Probability}

\section{Distribution}

Considering only one channel half of a straight channel with a wall at $y=d / 2$, the governing equation of the center-of-mass probability distribution $n\left(y_{c m}\right)$ in $y$-direction (perpendicular to flow direction) for steady-state conditions is given by (see chapter $5.5 .1)$

$$
-\frac{\tilde{K}\left(y_{c m}\right)}{\left(d / 2-y_{c m}\right)^{2}} n-\frac{\partial D\left(y_{c m}\right)}{\partial y_{c m}} n-D\left(y_{c m}\right) \frac{\partial n}{\partial y_{c m}}=0
$$

where

$$
\widetilde{K}\left(y_{c m}\right)=K_{0}\left(\frac{8 v_{0}}{d^{2}} y_{c m}\right)^{2 / 3}
$$

and

$$
D\left(y_{c m}\right)=\frac{D_{0} \cdot a}{\left(1+\left(b \frac{8 v_{0}}{d^{2}} y_{c m}\right)^{c}\right)} .
$$

$v_{0}$ is the maximal velocity at the channel center, $d$ is the channel width, and $y_{c m}$ is the center-of-mass position of the filaments. The constants $a=0.67 \mathrm{~mm}^{2} / \mathrm{s}, b=9.8 \cdot 10^{-3} \mathrm{~s}$, and $c=1.25$ are determined by fitting the measured diffusivity.

The differential equation can be solved by

$$
n\left(y_{c m}\right)=\frac{n_{0}}{a}\left(1+\left(b \frac{8 v_{0}}{d^{2}} y_{c m}\right)^{c}\right) \exp \left(-\frac{K_{0}}{D_{0}} g\left(y_{c m}\right)\right) \text {. }
$$


The function $g\left(y_{c m}\right)$ is given by $g\left(y_{c m}\right)=\int\left\{\left[\left(8 \cdot v_{0} \cdot y / d^{2}\right)^{2 / 3}\left(1+\left(9.8 \cdot 10^{-3} \cdot 8 \cdot v_{0} \cdot y / d^{2}\right)^{1.25}\right] /\left(0.67(d / 2-y)^{2}\right)\right\} \mathrm{d} y\right.$

$=\left(8 \cdot v_{0} / d^{2}\right)^{2 / 3}\left\{1.5 \cdot 1 / 3 \cdot\left\{3 \cdot y^{2 / 3} /(d / 2-y)+2 \cdot 3^{1 / 2} \cdot(d / 2)^{-1 / 3} \cdot \arctan \left[3^{-1 / 2}\left(1+2(2 \cdot y / d)^{1 / 3}\right]\right.\right.\right.$

$\left.+2 \cdot(d / 2)^{-1 / 3} \cdot \ln \left[(d / 2)^{1 / 3}-y^{1 / 3}\right]-(d / 2)^{-1 / 3} \cdot \ln \left[(d / 2)^{2 / 3}+(d / 2 \cdot y)^{1 / 3}+y^{2 / 3}\right]\right\}$

$+\left(8 \cdot 9.8 \cdot 10^{-3} \cdot v_{0} / d^{2}\right)^{1.25}\left\{288 \cdot y^{11 / 12}+264 \cdot d / 2 \cdot y^{11 / 12} /(d / 2-y)\right.$

- 506. $(d / 2)^{11 / 12} \cdot \arctan \left[3^{1 / 2}-2 \cdot(2 \cdot y / d)^{1 / 12}\right]$

$+506 \cdot 3^{1 / 2} \cdot(d / 2)^{11 / 12} \cdot \arctan \left[3^{-1 / 2}\left(-1+2(2 \cdot y / d)^{1 / 12}\right]\right.$

$+506 \cdot 3^{1 / 2} \cdot(d / 2)^{11 / 12} \cdot \arctan \left[3^{-1 / 2}\left(1+2(2 \cdot y / d)^{1 / 12}\right]+506 \cdot(d / 2)^{11 / 12} \cdot \arctan \left[3^{1 / 2}+2(2 \cdot y / d)^{1 / 12}\right]\right.$

$+1012 \cdot(d / 2)^{11 / 12} \cdot \arctan \left[(2 \cdot y / d)^{1 / 12}\right]$

$+506 \cdot(d / 2)^{11 / 12} \cdot \ln \left[(d / 2)^{1 / 12}-y^{1 / 12}\right]-506 \cdot(d / 2)^{11 / 12} \cdot \ln \left[(d / 2)^{1 / 12}+y^{1 / 12}\right]$

$+253 \cdot(d / 2)^{11 / 12} \cdot \ln \left[(d / 2)^{1 / 6}-(d / 2 y)^{1 / 12}+y^{1 / 6}\right]-253 \cdot(d / 2)^{11 / 12} \cdot \ln \left[(d / 2)^{1 / 6}+(d \cdot y / 2)^{1 / 12}+y^{1 / 6}\right]$

$+253 \cdot 3^{1 / 2} \cdot(d / 2)^{11 / 12} \cdot \ln \left[(d / 2)^{1 / 6}-3^{1 / 2}((d \cdot y / 2))^{1 / 12}+y^{1 / 6}\right]$

$\left.\left.-253 \cdot 3^{1 / 2} \cdot(d / 2)^{11 / 12} \cdot \ln \left[(d / 2)^{1 / 6}+3^{1 / 2}(d \cdot y / 2)^{1 / 12}+y^{1 / 6}\right]\right\} / 264\right\}$. 


\subsection{Bibliography}

1. D.N. Breslauer, P.J. Lee, and L.P. Lee, Microfluidics-based systems biology. Molecular Biosystems, 2006. 2(2): p. 97.

2. S.K. Sia and G.M. Whitesides, Microfluidic devices fabricated in poly(dimethylsiloxane) for biological studies. Electrophoresis, 2003. 24(21): p. 3563.

3. D.J. Beebe, G.A. Mensing, and G.M. Walker, Physics and applications of microfluidics in biology. Annual Review of Biomedical Engineering, 2002. 4: p. 261.

4. M.K. Runyon, B.L. Johnson-Kerner, and R.F. Ismagilov, Minimal functional model of hemostasis in a biomimetic microfluidic system. Angewandte Chemie, 2004. 43(12): p. 1531.

5. S. Köster, Biological matter in microfluidic environment - from single molecules to self-assembly. Doktorarbeit, Göttingen, 2006.

6. S. Köster, D. Steinhauser, and T. Pfohl, Brownian motion of actin filaments in confining microchannels. Journal of Physics-Condensed Matter, 2005. 17(49): p. S4091.

7. A.D. Stroock, S.K.W. Dertinger, A. Ajdari, I. Mezic, H.A. Stone, and G.M. Whitesides, Chaotic mixer for microchannels. Science, 2002. 295(5555): p. 647.

8. T.M. Squires and S.R. Quake, Microfluidics: Fluid physics at the nanoliter scale. Reviews of Modern Physics, 2005. 77(3): p. 977.

9. A. Groisman and V. Steinberg, Elastic turbulence in a polymer solution flow. Nature, 2000. 405(6782): p. 53.

10. A. Groisman and V. Steinberg, Elastic turbulence in curvilinear flows of polymer solutions. New Journal of Physics, 2004. 6: p. 29. 
11. D.E. Smith, H.P. Babcock, and S. Chu, Single-polymer dynamics in steady shear flow. Science, 1999. 283(5408): p. 1724.

12. P. LeDuc, C. Haber, G. Bao, and D. Wirtz, Dynamics of individual flexible polymers in a shear flow. Nature, 1999. 399(6736): p. 564.

13. P.S. Doyle, B. Ladoux, and J.L. Viovy, Dynamics of a tethered polymer in shear flow. Physical Review Letters, 2000. 84(20): p. 4769.

14. T.T. Perkins, D.E. Smith, and S. Chu, Single polymer dynamics in an elongational flow. Science, 1997. 276(5321): p. 2016.

15. Y.L. Chen, M.D. Graham, J.J. de Pablo, K. Jo, and D.C. Schwartz, DNA molecules in microfluidic oscillatory flow. Macromolecules, 2005. 38(15): p. 6680.

16. J.S. Hur, E.S.G. Shaqfeh, H.P. Babcock, and S. Chu, Dynamics and configurational fluctuations of single DNA molecules in linear mixed flows. Physical Review E, 2002. 6601: p. 1915.

17. P.J. Hagerman, Flexibility of DNA. Annual Review of Biophysics and Biophysical Chemistry, 1988. 17: p. 265.

18. D.E. Smith and S. Chu, Response of flexible polymers to a sudden elongational flow. Science, 1998. 281(5381): p. 1335.

19. S. Shrewsbury and C. Hallett, Salmeterol $100 \mu \mathrm{g}$ : an analysis of its tolerability in single- and chronic-dose studies. Annals of Allergy, Asthma, \& Immunology, 2001. 87(6): p. 465.

20. S. Gerashchenko and V. Steinberg, Statistics of tumbling of a single polymer molecule in shear flow. Physical Review Letters, 2006. 9603(3): p. 8304.

21. K.A. Dill and B.H. Zimm, A rheological seperator for very large DNA molecules. Nuceleic Acids Research, 1979. 7: p. 735. 
22. R.H. Shafer, Radial migration of DNA molecules in cylindrical flow. Biophysical Chemistry, 1974. 2: p. 185.

23. U.S. Agarwal, A. Dutta, and R.A. Mashelkar, Migration of macromolecules under flow - The physical origin and engineering implications. Chemical Engineering Science, 1994. 49(11): p. 1693.

24. D. Ausserré, J. Edwards, J. Lecourtier, H. Hervet, and F. Rondelez, Hydrodynamic thickening of depletion layers in colloidal solutions. Europhysics Letters, 1991. 14: p. 33.

25. L. Fang, H. Hu, and R.G. Larson, DNA configurations and concentration in shearing flow near a glass surface in a microchannel. Journal of Rheology, 2005. 49(1): p. 127.

26. H.B. Ma and M.D. Graham, Theory of shear-induced migration in dilute polymer solutions near solid boundaries. The Physics of Fluids, 2005. 17(8): p. 83103.

27. J.A. Millan, W.H. Jiang, M. Laradji, and Y.M. Wang, Pressure driven flow of polymer solutions in nanoscale slit pores. Journal of Chemical Physics, 2007. 126(12): p. 24905.

28. F.H. Garner and A.H. Nissab, Rheological properties of high viscosity solutions of long molecules. Nature, 1946. 158: p. 634.

29. R.M. Jendrejack, D.C. Schwartz, J.J. de Pablo, and M.D. Graham, Shearinduced migration in flowing polymer solutions: Simulation of long-chain DNA in microchannels. Journal of Chemical Physics, 2004. 120(13): p. 6315.

30. O.B. Usta, J.E. Butler, and A.J.C. Ladd, Flow-induced migration of polymers in dilute solution. The Physics of Fluids, 2006. 18(3): p. 31703. 
31. L.C. Nitsche and E.J. Hinch, Shear-induced lateral migration of Brownian rigid rods in parabolic channel flow. Journal of Fluid Mechanics, 1997. 332: p. 1.

32. G. Sekhon, R.C. Armstrong, and S.J. Myung, The origin of polymer migration in a nonhomogenuos flow field. Journal of Polymer Science, 1982. 20: p. 947.

33. P.O. Brunn and S. Chi, Macromolecules in nonhomogeneous flow fields: A general study for dumbbell model macromolocules. Rheologica Acta, 1984. 23: p. 163.

34. J. Käs, H. Strey, J.X. Tang, D. Finger, R. Ezzell, E. Sackmann, and P.A. Janmey, F-Actin, A model polymer for semiflexible chains in dilute, semidilute, and liquid crystalline solutions. Biophysical Journal, 1996. 70(2): p. 609.

35. S. Burlaco, P.A. Janmey, and J. Borejdo, Distribution of actin filament lengths measured by fluorescence microscopy. The American Physiological Society, 1992: p. C569.

36. A. Ott, M. Magnasco, A. Simon, and A. Libchaber, Measurement of the persistence length of polymerized actin using fluorescence microscopy. Physical Review A, 1993. 48(3): p. R1642.

37. E. Frey, Physics in cell biology: On the physics of biopolymers and molecular motors. Chemphyschem, 2002. 3(3): p. 270.

38. D. Stein, F.H.J. van der Heyden, W.J.A. Koopmans, and C. Dekker, Pressure-driven transport of confined DNA polymers in fluidic channels. Proceedings of the National Academy of Sciences of the United States of America, 2006. 103(43): p. 15853.

39. Y.M. Huang, M. Uppalapati, W.O. Hancock, and T.N. Jackson, Microtubule transport, concentration and alignment in enclosed microfluidic channels. Biomedical Microdevices, 2007. 9(2): p. 175. 
40. T. Kim, M.T. Kao, E. Meyhofer, and E.F. Hasselbrink, Biomolecular motordriven microtubule translocation in the presence of shear flow: analysis of redirection behaviours. Nanotechnology, 2007. 18(2): p. 25101.

41. H. Lodish, A. Berk, L. Zipursky, P. Matsudaira, D. Baltimore, and J. Darnell, Molecular Cell Biology. 2000, New York: W. H. Freeman.

42. P. Sheterline, J. Clayton, and J. Sparrow, Actin. 1998, Oxford: Oxford University Press.

43. T.D. Pollard and J.A. Cooper, Actin and actin-binding proteins. A critical evaluation of mechanisms and functions. Annual Review of Biochemistry, 1986. 55: p. 987.

44. J.X. Tang, T. Ito, T. Tao, P. Traub, and P.A. Janmey, Opposite effects of electrostatics and steric exclusion on bundle formation by F-actin and other filamentous polyelectrolytes. Biochemistry, 1997. 36(41): p. 12600.

45. J.X. Tang, S.E. Wong, P.T. Tran, and P.A. Janmey, Counterion induced bundle formation of rodlike polyelectrolytes. Berichte der Bunsen Gesellschaft für Physikalische Chemie, 1996. 100(6): p. 796.

46. P.A. Janmey, S. Hvidt, J. Käs, D. Lerche, A. Maggs, E. Sackmann, M. Schliwa, and T.P. Stossel, The mechanical properties of actin gels - elastic modulus and filament motions. Journal of Biological Chemistry, 1994. 269(51): p. 32503.

47. J.Y. Xu, W.H. Schwarz, J.A. Käs, T.P. Stossel, P.A. Janmey, and T.D. Pollard, Mechanical properties of actin filament networks depend on preparation, polymerization conditions, and storage of actin monomers. Biophysical Journal, 1998. 74(5): p. 2731.

48. K.S. Zaner, Physics of actin networks: 1. Rheology of semi-dilute F-actin. Biophysical Journal, 1995. 68(3): p. 1019. 
49. K.S. Zaner and T.P. Stossel, Physical basis of the rheologic properties of Factin. Journal of Biological Chemistry, 1983. 258(18): p. 11004.

50. W.H. Goldmann, M. Tempel, I. Sprenger, G. Isenberg, and R.M. Ezzell, Viscoelasticity of actin-gelsolin networks in the presence of filamin. European Journal of Biochemistry, 1997. 246(2): p. 373.

51. K.P. Janssen, L. Eichinger, P.A. Janmey, A.A. Noegel, M. Schliwa, W. Witke, and M. Schleicher, Viscoelastic properties of F-actin solutions in the presence of normal and mutated actin-binding proteins. Archives of Biochemistry \& Biophysics, 1996. 325(2): p. 183.

52. R. Ruddies, W.H. Goldmann, G. Isenberg, and E. Sackmann, The viscoelasticity of entangled actin networks - The influence of defects and modulation by talin and vinculin. European Biophysics Journal, 1993. 22(5): p. 309.

53. D.H. Wachsstock, W.H. Schwarz, and T.D. Pollard, Affinity of alpha-actinin for actin determines the structure and mechanical properties of actin filament gels. Biophysical Journal, 1993. 65(1): p. 205.

54. M. Hosek and J.X. Tang, Polymer-induced bundling of F-actin and the depletion force. Physical Review E, 2004. 6905(5): p. 1907.

55. A. Suzuki, M. Yamazaki, and T. Ito, Polymorphism of F-actin assembly. 1. A Quantitative phase diagram of F-actin. Biochemistry, 1996. 35(16): p. 5238.

56. I.Y. Wong, M.L. Gardel, D.R. Reichman, E.R. Weeks, M.T. Valentine, A.R. Bausch, and D.A. Weitz, Anomalous diffusion probes microstructure dynamics of entangled F-actin networks. Physical Review Letters, 2004. 9217(17): p. 8101.

57. J.H. Shin, M.L. Gardel, L. Mahadevan, P. Matsudaira, and D.A. Weitz, Relating microstructure to rheology of a bundled and cross-linked F-actin 
network in vitro. Proceedings of the National Academy of Sciences of the United States of America, 2004. 101(26): p. 9636.

58. T.E. Angelini, H. Liang, W. Wriggers, and G.C.L. Wong, Like-charge attraction between polyelectrolytes induced by counterion charge density waves. Proceedings of the National Academy of Sciences of the United States of America, 2003. 100(15): p. 8634.

59. T.E. Angelini, L.K. Sanders, H.J. Liang, W. Wriggers, J.X. Tang, and G.C.L. Wong, Structure and dynamics of condensed multivalent ions within polyelectrolyte bundles: a combined $x$-ray diffraction and solid-state NMR study. Journal of Physics-Condensed Matter, 2005. 17(14): p. S1123.

60. O. Pelletier, E. Pokidysheva, L.S. Hirst, N. Bouxsein, Y. Li, and C.R. Safinya, Structure of actin cross-linked with alpha-actinin: A network of bundles. Physical Review Letters, 2003. 9114(14): p. 8102.

61. G.C.L. Wong, A. Lin, J.X. Tang, Y. Li, P.A. Janmey, and C.R. Safinya, Lamellar phase of stacked two-dimensional rafts of actin filaments. Physical Review Letters, 2003.9101(1): p. 8103.

62. M.O. Steinmetz, A. Hoenger, P. Tittmann, K.H. Fuchs, H. Gross, and U. Aebi, An atomic model of crystalline actin tubes - combining electron microscopy with X-ray crystallography. Journal of Molecular Biology, 1998. 278(4): p. 703.

63. L.S. Hirst, R. Pynn, R.F. Bruinsma, and C.R. Safinya, Hierarchical selfassembly of actin bundle networks: Gels with surface protein skin layers. Journal of Chemical Physics, 2005. 123(10): p. 4902.

64. H.J. Kwon, A. Kakugo, K. Shikinaka, Y. Osada, and J.P. Gong, Morphology of actin assemblies in response to polycation and salts. Biomacromolecules, 2005. 6(6): p. 3005. 
65. A. Suzuki, T. Maeda, and T. Ito, Formation of liquid crystalline phase of actin filament solutions and its dependence on filament length as studied by optical birefingence. Biophysical Journal, 1991. 59: p. 25.

66. J. Viamontes and J.X. Tang, Continuous isotropic-nematic liquid crystalline transition of F-actin solutions. Physical Review E, 2003. 6704(4): p. 701.

67. M. Claessens, M. Bathe, E. Frey, and A.R. Bausch, Actin-binding proteins sensitively mediate F-actin bundle stiffness. Nature Materials, 2006. 5(9): p. 748.

68. W.H. Roos, A. Roth, J. Konle, H. Presting, E. Sackmann, and J.P. Spatz, Freely suspended actin cortex models on arrays of microfabricated pillars. ChemPhysChem, 2003. 4(8): p. 872.

69. Y.N. Xia and G.M. Whitesides, Soft lithography. Annual Review of Materials Science, 1998. 28: p. 153.

70. D.C. Duffy, J.C. McDonald, O.J.A. Schueller, and G.M. Whitesides, Rapid prototyping of microfluidic systems in poly(dimethylsiloxane). Analytical Chemistry, 1998. 70(23): p. 4974.

71. D. Qin, Y.N. Xia, and G.M. Whitesides, Rapid prototyping of complex structures with feature sizes larger than $20 \mu \mathrm{m}$. Advanced Materials, 1996. 8(11): p. 917.

72. H. Makamba, J.H. Kim, K. Lim, N. Park, and J.H. Hahn, Surface modification of poly(dimethylsiloxane) microchannels. Electrophoresis, 2003. 24(21): p. 3607.

73. S. Lee and J. Voros, An aqueous-based surface modification of poly(dimethylsiloxane) with poly(ethylene glycol) to prevent biofouling. Langmuir, 2005. 21(25): p. 11957.

74. S. Pasche, S.M. De Paul, J. Voros, N.D. Spencer, and M. Textor, Poly(Llysine)-graft-poly(ethylene glycol) assembled monolayers on niobium oxide 
surfaces: A quantitative study of the influence of polymer interfacial architecture on resistance to protein adsorption by ToF-SIMS and in situ OWLS. Langmuir, 2003. 19(22): p. 9216.

75. J.M. Watson and M.G. Baron, The behaviour of water in poly(dimethylsiloxane). Journal of Membrane Science, 1996. 110(1): p. 47.

76. E. Verneuil, A. Buguin, and P. Silberzan, Permeation-induced flows: Consequences for silicone-based microfluidics. Europhysics Letters, 2004. 68(3): p. 412.

77. $\quad$ http://www.ahf.de/asp/.

78. $\quad$ http://probes.invitrogen.com/servlets/spectra.

79. I.D. Cytoskeleton, USA, About actin. http://www.cytoskeleton.com/products/actins/aboutactin.html.

80. H. Isambert, P. Venier, A.C. Maggs, A. Fattoum, R. Kassab, D. Pantaloni, and M.F. Carlier, Flexibility of actin filaments derived from thermal fluctuations - Effect of bound nucleotide, Phalloidin, and muscle regulatory proteins. Journal of Biological Chemistry, 1995. 270(19): p. 11437.

81. J. Howard, Mechanics of motor proteins and the cytoskeleton. 2001, Sunderland: Sinauer Associates.

82. O. Kratky and G. Porod, Röntgenuntersuchung gelöster Fadenmoleküle. Recueil des travaux cimiques des Pays-Bas, 1949. 68: p. 1106.

83. L.D. Landau and E.M. Lifshitz, Statistical Physics. 1958, London: Pergamon Press.

84. G.A. Carri and M. Marucho, Statistical mechanics of worm-like polymers from a new generating function. Journal of Chemical Physics, 2004. 121(12): p. 6064 . 
85. J. Wilhelm and E. Frey, Radial distribution function of semiflexible polymers. Physical Review Letters, 1996. 77(12): p. 2581.

86. R.A. Granger, Fluid Mechanics. 1995, New York: Dover Publications.

87. H. Stöcker, Taschenbuch der Physik. 1998, Frankfurt: Verlag Harri Deutsch.

88. E. Guyon, J.-P. Hulin, L. Petit, and C.D. Mitescu, physical hydrodynamics. 2001, Oxford: Oxford University Press.

89. M. Doi and M.D. Edwards, The theory of polymers dynamics. 1999, Oxford: Oxford University Press.

90. R.G. Larson, The rheology of dilute solutions of flexible polymers: Progress and problems. Journal of Rheology, 2005. 49(1): p. 1.

91. D.E. Smith, H.P. Babcock, and S. Chu, Single-polymer dynamics in steady shear flow. Science, 1999. 283(5408): p. 1724-1727.

92. R.B. Bird, C.F. Curtiss, R.C. Amstrong, and O. Hassager, Dynamics of Polymeric Liquids-Fluid Mechanics. Vol. 1. 1987, New York: WileyInterscience.

93. M. Bixon, Polymer dynamics in solution. Annual Review of Physical Chemistry, 1976. 27: p. 65.

94. A. Celani, A. Puliafito, and K. Turitsyn, Polymers in linear shear flow: A numerical study. Europhysics Letters, 2005. 70(4): p. 464.

95. M. Chertkov, I. Kolokolov, V. Lebedev, and K. Turitsyn, Polymer statistics in a random flow with mean shear. Journal of Fluid Mechanics, 2005. 531: p. 251.

96. R.G. Winkler, Semiflexible polymers in shear flow. Physical Review Letters, 2006. 9712(12): p. 8301. 
97. R. Fåhraeus and T. Lindquist, The viscosity of blood in narrow capillary tubes. American Journal of Physiology, 1931. 96: p. 562.

98. A.D. Maude and R.L. Whitmore, The wall effect and the viscometry of suspensions. British Journal of Applied Physics, 1956. 7: p. 98.

99. G. Segré and A. Silberberg, Behaviour of macroscopic rigid spheres in Poiseuille flow Part 2. Experimental results and interpretation. Journal of Fluid Mechanics, 1962. 14: p. 136.

100. D. Di Carlo, D. Irimia, R.G. Tompkins, and M. Toner, Continuous inertial focusing, ordering, and separation of particles in microchannels. Proceedings of the National Academy of Sciences of the United States of America, 2007. 104(48): p. 18892.

101. F.P. Bretherton, The motion of rigid particles in a shear flow at low Reynolds number. Journal of Fluid Mechanics, 1962. 14: p. 284.

102. R.G. Cox and S.G. Mason, Suspended particles in fluid flow through tubes. Annual Review of Fluid Mechanics, 1971. 3: p. 291.

103. J.P. Matas, J.F. Morris, and E. Guazzelli, Lateral forces on a sphere. Revue de 1 Institut Francais du Petrole, 2004. 59(1): p. 59.

104. R.C. Jeffrey and J.R. Pearson, Particle motion in laminar vertical tube flow. Journal of Fluid Mechanics, 1965. 22: p. 721.

105. R. Eichorn and S. Small, Experiments on the lift and drag of spheres suspended in Poiseuille flow. Journal of Fluid Mechanics, 1964. 20: p. 513.

106. D.R. Oliver, Influence of particle rotation on radial migration in the Poiseuille flow of suspensions. Nature, 1962. 194: p. 1269.

107. A. Karnis, H.L. Goldsmith, and S.G. Mason, The flow of suspensions through tubes. V. Inertial effects. Canadian Journal of Chemical Engineering, 1966. 44: p. 181. 
108. H.L. Goldsmith and S.G. Mason, The microrheology of dispersions. Rheology: Theory and Applications, 1967. 4: p. 87.

109. E.S. Asmolov, The inertial lift on a spherical particle in a plane Poiseuille flow at large channel Reynolds number. Journal of Fluid Mechanics, 1999. 381: p. 63.

110. A.J. Hogg, The inertial migration of non-neutrally buoyant spherical particles in 2-dimensional shear flows. Journal of Fluid Mechanics, 1994. 272: p. 285.

111. B.P. Ho and L.G. Leal, Inertial migration of rigid spheres in 2-dimensional unidirectional flow. Journal of Fluid Mechanics, 1974. 65: p. 365.

112. P. Vasseur and R.G. Cox, The lateral migration of a spherical particle in two dimensional shear flows. Journal of Fluid Mechanics, 1976. 78: p. 385.

113. J.A. Schonberg and E.J. Hinch, Inertial migration of a sphere in Poiseuille flow. Journal of Fluid Mechanics, 1989. 203: p. 515.

114. C.J. Lin, J.H. Perry, and W.R. Schowalter, Simple shear flow round a rigid sphere: inertial effects and suspension rheology. Journal of Fluid Mechanics, 1970. 44: p. 1.

115. P.G. Saffman, The lift on a small sphere in a slow shear flow. Journal of Fluid Mechanics, 1965. 22: p. 385.

116. E.S. Asmolov, The inertial lift on a small particle in a weak-shear parabolic flow. Physics of Fluids, 2002. 14(1): p. 15.

117. P. Vasseur and R.G. Cox, The lateral migration of spherical particles sedimenting in a stagnant bounded fluid. Journal of Fluid Mechanics, 1977. 80: p. 561.

118. S.I. Rubinow and J.B. Keller, The transverse force on a spinning sphere moving in viscous fluid. Journal of Fluid Mechanics, 1961. 11: p. 447. 
119. L.G. Leal, Particle motions in a viscous fluid. Annual Reviews of Fluid Mechanics, 1980. 12: p. 435.

120. L.Y. Zeng, S. Balachandar, and P. Fischer, Wall-induced forces on a rigid sphere at finite Reynolds number. Journal of Fluid Mechanics, 2005. 536: p. 1.

121. J.P. Hernandez-Ortiz, H.B. Ma, J.J. Pablo, and M.D. Graham, Cross-streamline migration in confined flowing polymer solutions: Theory and simulation. The Physics of Fluids, 2006. 18(12): p. 23101.

122. R. Khare, M.D. Graham, and J.J. de Pablo, Cross-stream migration of flexible molecules in a nanochannel. Physical Review Letters, 2006. 9622(22): p. 4505.

123. D. Saintillan, E.S.G. Shaqfeh, and E. Darve, Effect of flexibility on the shear-induced migration of short-chain polymers in parabolic channel flow. Journal of Fluid Mechanics, 2006. 557: p. 297.

124. R.L. Schiek and E.S.G. Shaqfeh, Cross-streamline migration of slender Brownian fibers in plane Poiseuille flow. Journal of Fluid Mechanics, 1997. 332: p. 23.

125. P.O. Brunn, Polymer migration phenomena based on the general beadspring model for flexible polymer. Journal of Chemical Physics, 1984. 80(11): p. 5821.

126. C. Sendner and R.R. Netz, Shear-induced repulsion of a semiflexible polymer from the wall. Europhysics Letters, 2008. 81: p. 54006.

127. N. Phan-Thien, O. Manero, and L.G. Leal, A study of conformationdependent friction in a dumbbell model for dilute solutions. Rheologica Acta, 1984. 23: p. 151.

128. F.M. White, Viscous Fluid Flow. 1991, New York: McGraw-Hill. 
129. R.E. Teixeira, H.P. Babcock, E.S.G. Shaqfeh, and S. Chu, Shear thinning and tumbling dynamics of single polymers in the flow-gradient plane. Macromolecules, 2005. 38(2): p. 581.

130. J.S. Lee, E.S.G. Shaqfeh, and S.J. Muller, Dynamics of DNA tumbling in shear to rotational mixed flows: Pathways and periods. Physical Review E Statistical Physics, Plasmas, Fluids, \& Related Interdisciplinary, 2007. 75(4): p. 802 .

131. H. Stark, private communications.

132. R.M. Jendrejack, E.T. Dimalanta, D.C. Schwartz, M.D. Graham, and J.J. Pablo, DNA Dynamics in Microchannel. Physical Review Letters, 2003. 91(3).

133. $K_{0} / D_{0}=3000 ; v_{0}=0.23 \mathrm{~mm} / \mathrm{s}, 0.64 \mathrm{~mm} / \mathrm{s}, 1.7 \mathrm{~mm} / \mathrm{s}, 2.4 \mathrm{~mm} / \mathrm{s} ; n_{0}=2.3 \cdot 10^{-10}$, $1.9 \cdot 10^{-10}, 1.3 \cdot 10^{-10}, 1.2 \cdot 10^{-10}$.

134. S. Köster, H. Stark, T. Pfohl, and J. Kierfeld, Fluctuations of single confined actin filaments. Biophysical Reviews and Letters, 2007. 2(2): p. 155.

135. J.X. Tang and P.A. Janmey, The polyelectrolyte nature of F-actin and the mechanism of actin bundle formation. Journal of Biological Chemistry, 1996. 271(15): p. 8556.

136. A. Cebers, Z. Dogic, and P.A. Janmey, Counterion-mediated attraction and kinks on loops of semiflexible polyelectrolyte bundles. Physical Review Letters, 2006. 9624(24): p. 7801.

137. N. Gronbechjensen, R.J. Mashl, R.F. Bruinsma, and W.M. Gelbart, Counterion-induced attraction between rigid polyelectrolytes. Physical Review Letters, 1997. 78(12): p. 2477.

138. J.B. Knight, A. Vishwanath, J.P. Brody, and R.H. Austin, Hydrodynamic focusing on a silicon chip - Mixing nanoliters in microseconds. Physical Review Letters, 1998. 80(17): p. 3863. 
139. R. Dootz, A. Otten, S. Köster, B. Struth, and T. Pfohl, Evolution of DNA compaction in microchannels. Journal of Physics-Condensed Matter, 2006. 18: p. S639.

140. T. Pfohl, A. Otten, S. Köster, R. Dootz, B. Struth, and H.M. Evans, Highly packed and oriented DNA mesophases identified using in situ microfluidic X-ray microdiffraction. Biomacromolecules, 2007. 8(7): p. 2167.

141. S. Köster, J.B. Leach, B. Struth, T. Pfohl, and J.Y. Wong, Visualization of flow-aligned type I collagen self-assembly in tunable $p H$ gradients. Langmuir, 2007. 23(2): p. 357.

142. L. Pollack, M.W. Tate, A.C. Finnefrock, C. Kalidas, S. Trotter, N.C. Darnton, L. Lurio, R.H. Austin, C.A. Batt, S.M. Gruner, and S.G.J. Mochrie, Time resolved collapse of a folding protein observed with small angle $x$-ray scattering. Physical Review Letters, 2001. 86(21): p. 4962.

143. P.W. Atkins, Physikalische Chemie. 2.Edition ed. 1990, Berlin: Wiley-VCH.

144. G.H. Lai, R. Coridan, O.V. Zribi, R. Golestanian, and G.C.L. Wong, Evolution of growth modes for polyelectrolyte bundles. Physical Review Letters, 2007. 98(18): p. 7802.

145. H.J. Kwon, Y. Tanaka, A. Kakugo, K. Shikinaka, H. Furukawa, Y. Osada, and J.P. Gong, Anisotropic nucleation growth of actin bundle: A model for determining the well-defined thickness of bundles. Biochemistry, 2006. 45(34): p. 10313.

146. D. Biron and E. Moses, The effect of alpha-actinin on the length distribution of F-actin. Biophysical Journal, 2004. 86(5): p. 3284.

147. D. Biron, E. Moses, I. Borukhov, and S.A. Safran, Inter-filament attractions narrow the length distribution of actin filaments. Europhysics Letters, 2006. 73(3): p. 464. 
148. O.G. Berg and P.H. von Hippel, Diffusion-controlled macromolecular interactions. Annual Review of Biophysics and Biophysical Chemistry, 1985. 14: p. 131.

149. M.-C. Renoult, Study of the actin-bundling with $\alpha$-actinin in microflow by FRET microscopy. 2007, practicum report, Max-Planck Institut für Dynamik und Selbstorganisation, Göttingen. 


\section{Publications}

Electrowetting: A convenient way to switchable wettability patterns

Frieder Mugele, Anke Klingner, Jürgen Bührle, Dagmar Steinhauser, and Stephan Herminghaus.

2005, Journal of Physics-Condensed Matter, 17(9), p. S559.

Brownian motion of actin filaments in confining microchannels

Sarah Köster, Dagmar Steinhauser, and Thomas Pfohl.

2005, Journal of Physics-Condensed Matter, 17(49), p. S4091.

Microfluidic mixing through electrowetting-induced droplet oscillations

Frieder Mugele, Jean-Christophe Baret, and Dagmar Steinhauser.

2006, Applied Physics Letters 88, p. 204106.

Cross-streamline migration of semiflexible actin filaments

Dagmar Steinhauser, Holger Stark, Sarah Köster, and Thomas Pfohl.

In preparation.

Semiflexible polymers in microflow

Dagmar Steinhauser, Holger Stark, and Thomas Pfohl.

In preparation. 



\section{Danksagung}

An dieser Stelle möchte ich mich bei all denen bedanken, die mich während meiner Promotion unterstützt und zum Gelingen dieser Arbeit beigetragen haben. Mein besonderer Dank gilt hierbei Thomas Pfohl für die intensive und sehr gute Betreuung. Zahlreiche Diskussionen, gemeinsames Entwickeln neuer Ideen und hilfreiche Vorschläge während der gesamten Promotionszeit waren die Grundlage für diese Arbeit. Zusätzlich zu den wissenschaftlichen Hilfestellungen hat Thomas mich auch mit seiner positiven und motivierenden Art immer unterstützt und bei Misserfolgen aufgemuntert und weitergeholfen.

Stephan Herminghaus danke ich für die fachliche Anregungen und die Ermöglichung meine Arbeit am Max-Planck-Institut für Dynamik und Selbstorganisation durchführen zu können. Die gute Ausstattung und die tollen Möglichkeiten des Instituts haben die Verwirklichung und Durchführung vieler Experimente erleichtert. Bei Tim Salditt möchte ich mich für die Übernahme des Gutachtens bedanken, sowie auch für sein wissenschaftliches Interesse an meiner Arbeit und die Einladung zur Winterschule. Des Weiteren gilt mein Dank Rainer Ulbrich, Marcus Müller, Markus Münzenberg und Reiner Kree für die freundliche Bereiterklärung der Mitgliedschaft im Prüfungskomittee.

Der „Arbeitsgruppe Pfohl“, bestehend aus Eric Stellamanns, Heather Evans, Rolf Dootz, Sarah Köster, Semra Öztürk und Sravanti Uppaluri, möchte ich für die gute Zusammenarbeit, gegenseitige Unterstützung und tolle Athmosphäre danken. Die gemeinsamen Unternehmungen und Erlebnisse werden mir in guter Erinnerung bleiben. Im Besonderen möchte ich mich bei Heather für die Korrekturen an dieser Arbeit bedanken. Ebenso gilt mein Dank auch Sarah, deren Erfahrungen und vorangegangene Arbeiten rund um das Aktin für mich sehr hilfreich waren. Marie-Charlotte Renoult möchte ich danken für die Zusammenarbeit während ihres Praktikums bezüglich der FRET-Mikroskopie von Aktinbündeln.

Bei Holger Stark bedanke ich mich für Diskussionen und die Hilfe zur theoretischen Beschreibung der Experimente, insbesondere der Biegung der Aktinfilamente im Fluss. Den Technikern Wolf Keiderling und Udo Krafft gilt mein Dank für die meist schnelle und sehr zuverlässige Hilfe bei Aufbauten und technischen Problemen. Ohne diese Hilfe wären manche Experimente nur schwierig durchzuführen gewesen.

Abschließend möchte ich noch meinen Eltern und Geschwistern für ihre Unterstützung und Aufmunterungen herzlich danken. 



\section{Lebenslauf}

\section{Persönliche Angaben}

Name:

Dagmar Regine Steinhauser

Geburtstag:

22. Juni 1979

Geburtsort:

Schwäbisch Hall

Staatsangehörigkeit:

deutsch

\section{Ausbildung}

Seit $06 / 2004$

Promotion am Max-Planck-Institut für Dynamik und Selbstorganisation in Göttingen

$02 / 2004$

Diplom in Physik

$03 / 2003-02 / 2004$

Diplomarbeit in der Abteilung Angewandte Physik der Universität Ulm

$07 / 2000-02 / 2004$

Studium der Physik an der Universität Ulm

$07 / 2000$

Wechsel in der Diplomstudiengang Physik

$10 / 1998-07 / 2000$

Diplomstudium der Wirtschaftsphysik an der Universität Ulm

$06 / 1998$

Abitur

08/1989-06/1998 Besuch des Gymnasiums in Gerabronn

08/1985 - 07/1989 Besuch der Grundschule in Langenburg 
